\title{
Mechanics \& Dynamics of the Primary Cilium
}

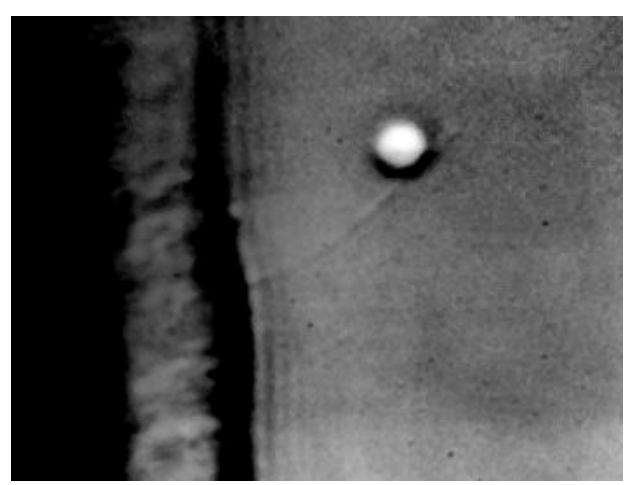

Dissertation

zur Erlangung des mathematisch-naturwissenschaftlichen Doktogrades

"Doctor rerum naturalium"

der Georg-August-Universität Göttingen

vorgelegt von

Christopher Guy Battle

aus Vancouver, Kanada

Göttingen 2013 
Prof. Dr. Christoph F. Schmidt (Referent)

Drittes Physikalisches Institut, Georg-August-Universität

\author{
Dr. Andreas Neef (Referent) \\ Abteilung Nichtlineare Dynamik, Max-Planck-Institut für Dynamik und \\ Selbstorganization \\ Prof. Dr. Marcus Müller \\ Institut für Theoretische Physik, Georg-August-Universität
}

Tag der mündlichen Prüfung:

25. Juni, 2013 
I hereby declare that this dissertation has been written independently and with no other sources or aids than those quoted. 
To Amélie 


\begin{abstract}
Recent studies have shown that the primary cilium, long thought to be a vestigial organelle with no function, is involved in a multitude of sensory functions. One example, interesting from both biophysical and medical standpoints, is the primary cilium of kidney epithelial cells, which acts as a mechanosensitive flow sensor. The mechanical properties of these non-motile, microtubule-based cilia and their anchorage to the cell cytoskeleton are important for understanding the cell's mechanosensitive response, which is mediated by the cilium. In order to explore this response, we built an epifluorescence microscope with optical trapping and patch clamping capabilities, and describe preliminary results. To probe the mechanical properties, cellular anchoring conditions, and dynamics of the cilia of Madin-Darby canine kidney epithelial cells, we built high-quality differential interference contrast microscopy capabilities into an existing dual-optical trap setup and wrote a custom MATLAB algorithm to track ciliia dynamics. Experimentally determined values for the flexural rigidity of primary cilia are reported, as well as evidence for a hinged boundary condition at the cellular attachment point. Additionally, we find evidence of active, non-equilibrium behavior in the cilium's fluctuation dynamics.
\end{abstract}




\section{Acknowledgements}

First and foremost, I'd like to thank my adviser, Christoph Schmidt, for his patience and guidance throughout my doctoral research. Without his input of time, ideas, and expertise this project wouldn't have progressed at all and the many difficulties along the way wouldn't have been overcome. Knowing whether to keep progressing down a particular path or whether to strike off in a new direction is an important part of research, and his advice on this point made this project successful.

I'd also like to thank my thesis committee members, Andreas Neef and Marcus Müller, for their support and advice. Their suggestions for project goals and improvements were always helpful and well-thought out. I'd like to thank Andreas Neef in particular for help, advice, and technical expertise on all things electrophysiological.

This thesis also presents collaborative results, and so of course I'd like to thank my collaborators Carolyn Ott from NIH, Andrew Woehler from the MPI-BPC, and Andreas Neef (again) from the MPI-DS for their contributions to this work. Their work, especially Carolyn's, has improved and enriched the results presented here.

A good working environment makes the long hours of a PhD much more bearable, and for that I'd like to thank the entire DPI institute, especially Marcel Bremerich, Christina Thiede, Sai Li, Frederic Eghiaian, Christina Jayachandran, Til Driehorst, Connie, Adam Walker, André Düselder, Florian Schlosser, Miquel Banches i Piqué, Alok Weßel, Christopher Dilip, Ulrich Fromme, Carina Wollnik, Kerstin van Roden, Ulrike Schulz, Charlotte Willms (thanks for the fluorescent cilia!), Dieter Klopfenstein, Florian Rehfeldt, Meenakshi Prabhune, Theresa Kaufeld, Alice Wiesbaum, Nikta Fakhri, Nuria Gavara, Iwan Schaap, Paula Sanchez Baeza, Knut Heidemann, Elke Zech, Nicole Rehbein, Ursula Hahn-Wörgötter, Thomas Geiling, Dietrich Hille, Karl Lautscham, and Markus Schönekess. I'd also like to thank Jörg Enderlein for helpful discussions about the depolarization effects discussed in Chapter 3.

Editing and correcting something as long as a dissertation is a monstrous task, and I'd like to thank Katarzyna Tych and Andrew Woehler very much for their help. I owe you both dinner and some $\mathrm{G} \& \mathrm{Ts}$.

A thesis can never be written without a support group, so a shout out to the Göttingen crew: Andrea Maddalena, Giovanni Marelli, Ahmed El Hady, Andrew Woehler (yet another shout out), Jonas Barth, Matthieu Hammer, Liam Tuffy, Sanaz "my Nizzle" Bahari-Javan, Derya Akad, Natalia Manrique, Iliana Panou, Nikolai Chapochnikov (thanks for the bike, runs great), Andonia Petovka, Tina Ghelani, Adam Roe, Despoina Evangelakou, Alwaleed Alkhaja, Stéphanie Bonnet, Sebastian Kiehlmann, Marius Eilbrecht, Phillip Kroehn, Mirko Lukovic, Magda Skowronski, Marta Rychlewski, Vesna Gagic, and if there's anyone I forgot, I'm sorry, I'll buy you a beer.

Spasiba za vse minya tschucktscha, tseluyu. 


\section{Contents}

$\begin{array}{ll}\text { Contents } & 1\end{array}$

1 Introduction $\quad 3$

2 Construction of an epifluorescence microscope/optical trap/ $\begin{array}{ll}\text { patch clamp setup } & 9\end{array}$

3 Incorporation of DIC microscopy into a dual-optical trap 45

4 Tracking DIC line images $\quad 63$

5 Experimental methods $\quad 83$

$\begin{array}{lll}6 & \text { Results } & 91\end{array}$

$\begin{array}{lll}7 & \text { Discussion } & 147\end{array}$

$\begin{array}{lll}\text { I Appendices } & 159\end{array}$

$\begin{array}{ll}\text { A Protocols } & 161\end{array}$

B Influence of DIC prism orientation on crosstalk 167

$\begin{array}{ll}\text { Bibliography } & 169\end{array}$ 


\section{Chapter 1}

\section{Introduction}

The hero of this story is the primary cilium, pictured in Figure 1.1. The primary cilium is a ubiquitous, microtubule-based organelle that can be found in almost every eukaryotic cell at some point in its life cycle [1]. Primary cilia are slender, hair-like appendages that generally extend from the apical cell surface, though in some cases are contained inside the cell in a recessed invagination [2]. The cilium core structure, called the axoneme, is made up of nine microtubule doublets arranged in a ring and is surrounded by the plasma membrane. Cilia are characterized as either motile or non-motile, with the majority of primary cilia falling into the second category. Motile cilia, such as the mucus transporting cilia in the respiratory tract and sperm flagella, have a so-called " $9+2$ " axonemal structure in which the microtubule doublets surround a central microtubule pair. In addition to the central microtubule pair, motile cilia have dynein motor proteins which power motility by generating relative sliding between microtubule doublets [3]. Primary cilia, on the other hand, have a " $9+0$ " axonemal structure in which the central microtubule pair is missing. They also lack the motile cilium's dyneins and associated proteins implicated in motility (e.g. nexin, radial spokes). Long believed to be vestigial organelles, primary cilia were ignored for the most part by cell biologists until ciliary defects were found to underly medical disorders such as Bardet-Biedl syndrome [4], polycystic kidney disease [5], and left-right asymmetry defects (situs inversus) in developing embryos [6]. Research over the last 15 years has shown the primary cilium to be a crucial sensory organelle, mediating mechanosensation, olfaction, photosensation, osmosensation, chemosensation, extracellular matrix signaling, and the developmental signaling pathways Hedgehog and Wnt [1]. Due to their sensory function, a wide range of receptors and ion channels are enriched in the cilium, such as platlet-derived growth factor, somatostatin receptor 3 , serotonin receptor 5 , polycystin $1 \& 2$, and components of the Hedgehog and Wnt pathways [7, 8]. Disease-causing dysfunctions in primary cilia, termed "ciliopathies", have been proposed to underly a wide variety of pathologies such as cystic diseases, skeletal patterning defects, anosmia, 

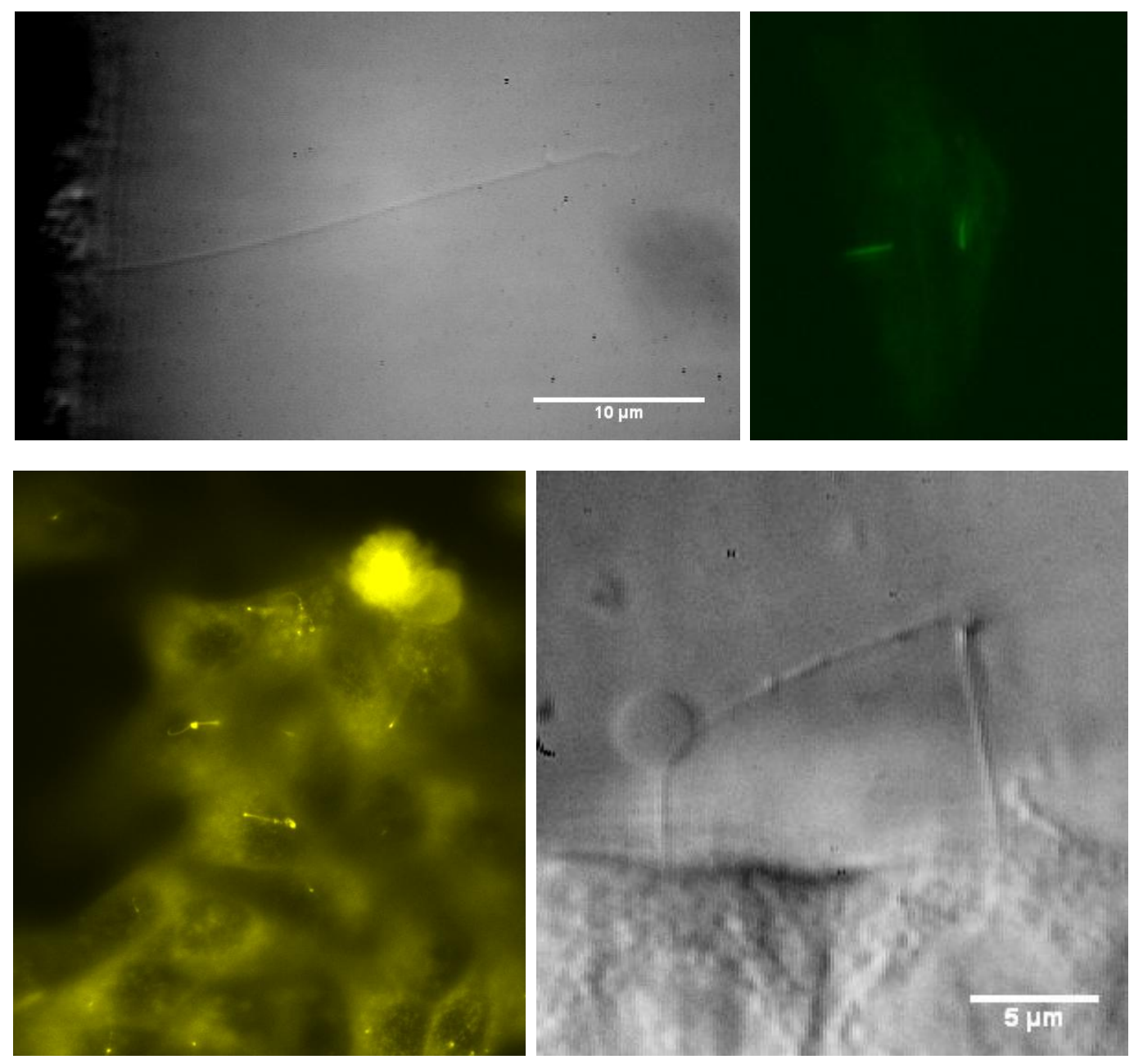

Figure 1.1: Micrographs of primary cilia. Top left: Long primary cilium extending from an MDCK cell, visualized from the side with DIC microscopy. Top right: Fluorescent human retinal pigment epithlial (RPE1) cells transfected with a green fluorescent ADP-ribosylation factor-like protein 13B (ARL13B-GFP), a protein which plays a role in cilia maintenance and localizes to the cilium (the transfected RPE1 cells were a kind gift from Hiroaki Ishikawa and the Marshall lab at the University of San Francisco). Bottom left: Confluent MDCK cells transfected with yellow fluorescent Smoothened protein (Smo-YFP). Smoothened plays a role in the hedgehog signaling pathway and localizes to the primary cilium. Bottom right: Cilia bridge formed by two adjacent primary cilium from MDCK cells, visualized from the side with DIC microscopy. It has been speculated that these bridges may play a role in intercellular communication [9]. 

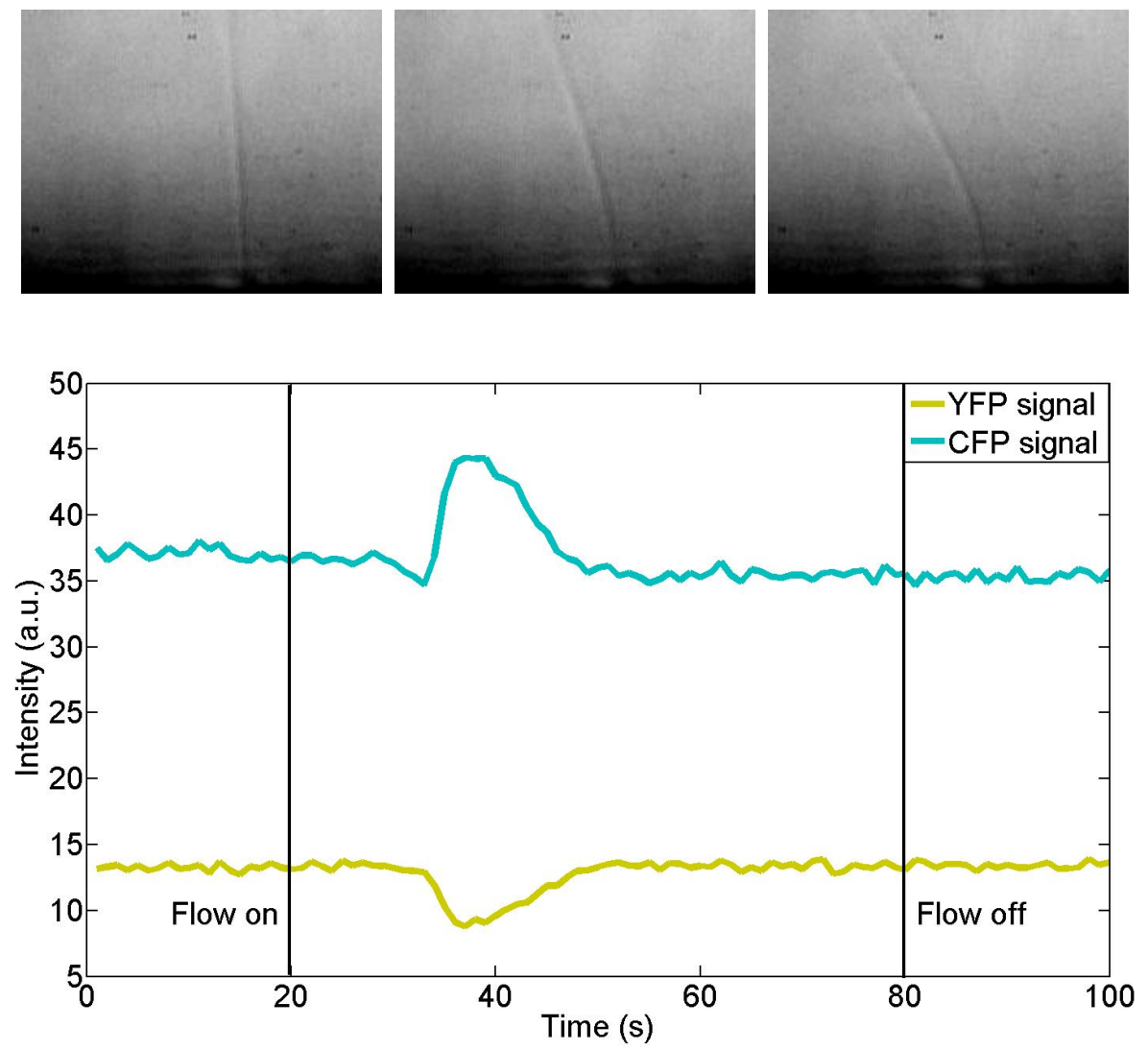

Figure 1.2: Cilium bending and mechanosensitive response. Top row: Primary cilium from an MDCK cell bending in increasing flow. Bottom: Calcium response of an MDCK cell to flow. Flow is initiated and 10-15 seconds later there is a peak in intracellular calcium levels. The graph shows the response of Yellow cameleon 3.60 (YC 3.60), a calcium sensitive Förster resonance energy transfer (FRET) indicator which was expressed in transfected MDCK cells [10]. Upon calcium binding, YC 3.60 undergoes a conformational change which brings the CFP (donor) and YFP (acceptor) molecules closer together. This change increases FRET efficiency, leading to a decrease in the CFP fluorescence intensity and a concomitant increase in the YFP fluorescence intensity. Note that the cilium stills and the graph are not from the same experiment. Calcium imaging experiments performed in collaboration with Andrew Woehler.

hydrocephalus, diabetes, skin diseases, atherosclerosis, and cancer, among others [7]. Given their importance as signaling centers, primary cilia have become a subject of very active research in the biological and medical communities.

In this work we will explore the mechanical properties and dynamics of the pri- 


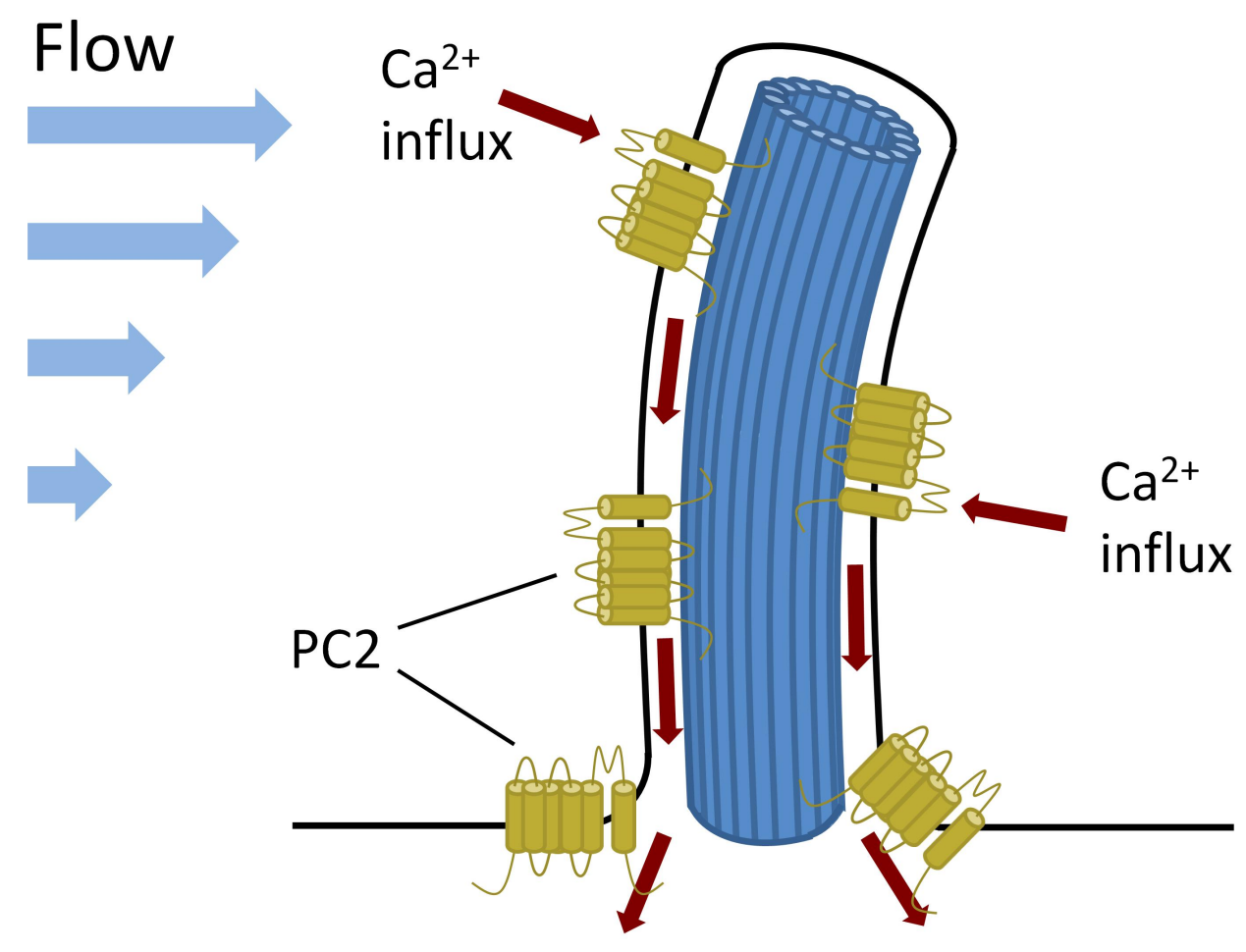

Figure 1.3: Diagram of primary cilium mechanosensitive response to flow. Cilium bending in flow is believed to gate mechanosensitive channels, primarily $\mathrm{PC} 2$, which leads to calcium influx and downstream signaling.

mary cilia of Madin-Darby canine kidney (MDCK II) cells ${ }^{1}$. MDCK cells are an epithelial cell line derived from the kidney of a cocker spaniel, commonly used as a general model for epithelial cells. More interesting from our standpoint are the primary cilia of MDCK cells, which extend from the apical side of the kidney epithelium into the nephron and act as microscopic flow sensors. This mechanosensing process is necessary for healthy organ maintenance, and defects in it lead to the aforementioned polycystic kidney disease (PKD) [11].

The top row of Figure 1.2 shows a series of stills depicting an MDCK primary cilium bending in flow. Ciliated MDCK cells have been shown to have a calcium response in the presence of flow $[12,13,14]$, an example of which is shown in the bottom panel of Fig. 1.2. A peak calcium response is seen roughly 15 seconds after the initiation of flow, in line with previous findings $[13,14]$. This calcium response has been tied to the activity of the mechanosensitive ion channel polycystin-2 (PC2) and

\footnotetext{
${ }^{1}$ We will often for brevity write "MDCK" to mean MDCK II cells, which are the only type of cells investigated in this work
} 
its partner polycystin-1 (PC1), so named due to their role in PKD. PC2 is a calcium permeable, non-selective, transient receptor potential (TRP) cation channel with six transmembrane domains, while PC1 is an 11 transmembrane domain protein with a large extracellular N-terminus which may play a role in mechanosensation $[15,16]$. Taken together the two proteins are thought to associate via their C-termini to form a complex in which PC1 acts as a "receptor" which modulates PC2 ion channel activity [16]. Figure 1.3 illustrates the current primary cilium/calcium signaling dogma: Fluid flow bends the cilium, leading to stress in the membrane which gates embedded PC2 channels (associated to PC1, not shown) and leads to calcium influx [14]. This initial calcium response is amplified by calcium release from inositol 1,4,5-trisphosphate $\left(\mathrm{IP}_{3}\right)$ sensitive stores, which then spreads to adjacent cells via secondary messengers diffusing through tight junctions [12]. The final downstream effect of this signaling cascade is not known, but it may regulate ion transport across the epithelium, thus coupling it to the flow rate in the nephron [8].

While the research to date has provided a rough outline of mechanosensing in MDCK primary cilia, relatively little is known about the biophysical details of this sensor. In this vein we aim to answer three questions in this work: What is the bending stiffness of the primary cilium? What is the boundary condition at the cilium attachment point to the cell? Are cilium position fluctuations, ever-present even in the absence of flow, actively driven or are they the result of thermal fluctuations?

Mechanical parameters, most importantly the cilium's bending stiffness, can be expected to govern mechanosensing, affecting variables such as the amount of stretch/compression in the membrane at a given flow rate, calcium response lag times (via the build-up rate of membrane tension), and force transmission to the cilium base ${ }^{2}$. There has been remarkably little research done to characterize primary cilium bending stiffness, EI, with Reference [17] being the only study to address it until two studies appeared last year [18, 19]. All three of these studies used flow-induced bending to measure $E I$, while we present an alternate methodology using optical traps, which have the advantage of delivering force locally in a precise, controlled manner.

The boundary condition (BC) at the cilium attachment point impacts the calculation of $E I$ and the scaling behavior of cilium fluctuations, but it is interesting in its own right as it provides clues to the confinement of the cilium base in the cell. It reflects cilium anchoring and affects its deformation profile under flow. The attachment $\mathrm{BC}$ is almost universally considered to be clamped (i.e. the cilium position and its first derivative with respect to position are constant), but we will challenge this assumption in this work.

Although primary cilia are considered to be non-motile, they still exhibit position

\footnotetext{
${ }^{2}$ There is some debate about whether the mechanosensitive response is mediated by channels located in the ciliary membrane or by channels located at the cilium base. We will come back to this point in the Discussion chapter.
} 
fluctuations in the absence of flow. While they are microscopic objects subject to Brownian motion, they are also attached to a highly non-equilibrium system, i.e. the cell. As such it is possible that the fluctuations which they exhibit aren't principally driven by thermal motion, but rather by active cellular motion, a possibility which to our knowledge hasn't been explored. Evidence of active fluctuations opens the possibility that they may play some functional role.

This work proceeds as follows: In the second chapter, construction of an epifluorescence microscope/optical trap/patch clamp setup is described. This setup was designed to perform measurements characterizing the electrophysiology of the mechanosensitive response of MDCK cells. The third chapter describes modifying a microscope with dual-optical trapping capabilities to perform high-quality differential interference contrast (DIC) microscopy. High-quality DIC was crucial for the optical trapping and fluctuation tracking experiments described in the Results chapter. A manuscript detailing this method was accepted for publication in the journal Review of Scientific Instruments. In the fourth chapter we describe the customwritten tracking algorithm that underpins the fluctuation analysis described in the Results chapter. In the fifth chapter we detail the experimental materials and methods used in the experiments subsequently presented and in the sixth we present the results of our optical trapping and fluctuation tracking experiments, as well as collaborative results. Finally, the seventh chapter discusses our results in the context of current research and outlines possibilities for future work. 


\section{Chapter 2}

\section{Construction of an epifluorescence microscope/optical trap/ patch clamp setup}

In order to probe the mechanical characteristics and mechanosensitive response of an object like the primary cilium, there are three technical hurdles that we have to clear: we must be able to visualize an object with one dimension on the scale of micrometers and another on the scale of hundreds of nanometers, we must be able to apply appropriate forces to such an object in a quantitative and reproducible way, and we must be able to measure the electrochemical response of the cell upon mechanical perturbation. It is with these design considerations in mind that we built the epifluorescence/optical trap/patch clamp setup described below. Optical trapping, the creation of a $3 \mathrm{D}$ trapping potential for dielectric objects via radiation pressure, provides a flexible and precise way to manipulate the primary cilium. Our decision to combine optical trapping with fluorescence microscopy was driven by two factors: first, the clear imaging benefits afforded by fluorescence (e.g. contrast, resolvability), and second, the design requirements of another experiment to be performed using the setup. The second experiment aims to measure the characteristics of mechanosensitive ion channels embedded in substrate-supported lipid bilayers. Since the bilayers are spread on opaque substrates, transmitted light microscopy can't be used to view them, making fluorescence microscopy the obvious alternative. Similarly, we chose to build patch-clamping capabilities into the set-up for two reasons: in hopes of recording the cell's electrical response upon mechanical perturbation of the cilium and in order to record single-channel ion currents in the supported-bilayer experiment.

In this chapter we detail the construction of the above setup. We start with a brief review of optical trapping and fluorescence microscopy, followed by an overview of the operating principles behind various pieces of instrumentation, then give a 
technical description of the setup and its performance, ending with a discussion of its relation to the experiments described later in the text. Though the setup has the capability to perform electrophysiological experiments, we avoid giving a review of the subject, as we only use these capabilities in a small part of the work presented. The interested reader is referred to Reference [20], "the Electrophysiology Bible".

\section{Optical trapping}

Optical traps, or optical tweezers, (OT) have become a mainstay in biophysics since Arthur Ashkin's discovery more than 25 years ago that dielectric objects could be trapped and manipulated using laser light [21]. The ability to stably trap dielectric particles in three dimensions with a laser focused through a high numerical aperture (NA) microscope objective has found a place in fields as diverse as the micromechanics of mechanoenzymes and biopolymers [22, 23, 24, 25], the microrheology of complex fluids [26, 27, 28], and free energy measurements of molecular kinetics [29]. In biophysics applications one often traps silica or polystyrene microspheres, typically ranging from $0.5-4.0 \mu \mathrm{m}$ in diameter, and attaches them to the biological object of interest via nonspecific or specific (e.g. fibronectin-integrin, antibody) binding. Then one pulls on the attached sphere, or "handle", by moving either the stage or trap, thus exerting a force on the object of interest. The forces exerted tend to be in the piconewton to tens of piconewtons range, and the forces/displacements created in this manner can be measured with the aid of split photodiodes and laser interferometry [30]. Near-infrared lasers are typically used for trapping, as it has been shown that they cause minimal radiation damage to biological specimens [31].

The main principle behind optical trapping is that, for objects of an appropriate geometry and refractive index apropos their environment, a force balance can be achieved between the refracted and scattered rays of incident radiation. The origin of this force balance is relatively easy to understand for a spherical particle in the geometrical optics limit (where the size of the dielectric object $\gg \lambda$, the wavelength of the incident light) and the Rayleigh limit (where the size of the dielectric object $\ll$ $\lambda)$, an explanation of which is presented below, following the treatment in Refs. [31] $\&[32]$.

In the geometric optics limit, or Mie regime, the trapping force can be understood in terms of the momentum transfer of light refracted through a microsphere of a refractive index $n$ higher than that of the surrounding media. When light is refracted by the sphere it changes momentum, and conservation of momentum implies that an equal and opposite change in momentum is imparted to the sphere, giving rise to a force by Newton's 2nd Law. When a gradient of light exists, as is the case in the usual Gaussian beam profile of a $\mathrm{TEM}_{00}$ laser, the vector sum of the rays generates a force imbalance which pushes the sphere towards higher intensity regions. The 


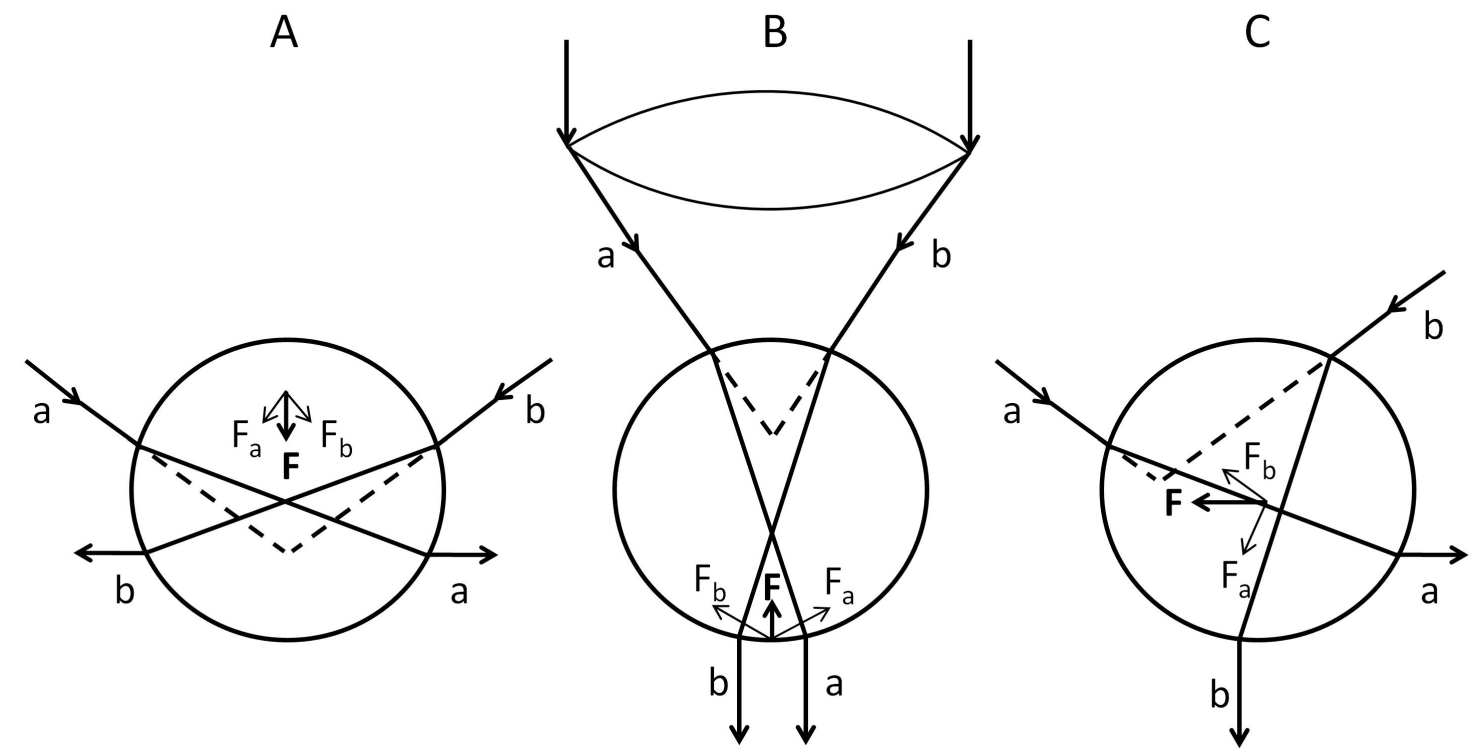

Figure 2.1: Diagram of scattering versus refractive force balance in the geometrical optics regime for A) a sphere above the beam focus, B) a sphere below the beam focus, and C) a sphere displaced to the right of the beam focus. After Ref. [32].

force from a Gaussian beam is thus directed toward the laser focus, which can create stable trapping if the gradient force arising from refracted rays is stronger than the scattering force. Figure 2.1 shows qualitative force diagrams for the gradient force for various bead positions in a beam focused by a high NA lens. Figure 2.1A \& 2.1B depict microspheres positioned left-right symmetrically in the beam line but above and below the focus, respectively. In case A, the refraction of the beams to a crossing point above the focus generates a downward force on the bead (in addition to the implied scattering force) by conservation of momentum, with the left-right components canceling out due to the symmetry of the beam. In case B, the incident light rays are refracted to a crossing point below the laser focus, which generates an upward force on the bead, with the left-right components again canceling out due to symmetry. Figure $2.1 \mathrm{C}$ shows a microsphere displaced to the right of the focus, and again momentum transfer from the refracted light gives a net leftward force, pulling the bead back toward the laser focus.

While the preceding paragraph gives an intuitive description of how trapping forces arise, the real situation is somewhat more complicated. Ashkin gave a quantitative account of the trapping force in Ref. [32], including the effects of polarization and all the internally reflected and refracted beams, which is exact in the geometric optics approximation. The force, $\mathbf{F}$, of a single ray of power $\mathrm{P}$ incident upon a sphere (see Figure 2.2) is given by 


$$
\begin{aligned}
\mathbf{F}=\frac{n_{m} \mathrm{P}}{c}\{1+\mathrm{R} \cos 2 \theta & \left.-\frac{\mathrm{T}^{2}[\sin (2 \theta-2 \epsilon)+\mathrm{R} \cos 2 \theta]}{1+\mathrm{R}^{2}+\mathrm{R} \cos 2 \epsilon}\right\} \hat{\mathbf{k}} \\
& +\frac{n_{m} \mathrm{P}}{c}\left\{\mathrm{R} \sin 2 \theta-\frac{\mathrm{T}^{2}[\sin (2 \theta-2 \epsilon)+\mathrm{R} \sin 2 \theta]}{1+\mathrm{R}^{2}+\mathrm{R} \cos 2 \epsilon}\right\} \hat{\mathbf{i}}
\end{aligned}
$$

where $\mathrm{R}$ and $\mathrm{T}$ are the Fresnel coefficients for reflection and transmission, $n_{m}$ is the refractive index of the surrounding medium, $c$ is the speed of light in a vacuum, $\hat{\mathbf{k}}$ and $\hat{\mathbf{i}}$ are unit vectors parallel and perpendicular to the direction of the incident ray, $\theta$ is the angle of incidence, and $\epsilon$ is the angle of refraction. The first term, parallel to the direction of the incident beam, gives the scattering force while the second term, perpendicular to the incident beam, gives the gradient force. Stable trapping necessitates minimizing the scattering force in relation to the gradient force, which means maximizing the gradient force in the $-\mathrm{z}$ direction (per Figure 2.2). Equation 2.1 increases non-monotonically with increasing $\theta$ and the gradient force attains a maximum with respect to the scattering force for higher angle rays (e.g. $\approx 60^{\circ}$ ). Since higher angle rays contribute more to the gradient force, it is common practice to expand the trapping beam so that it overfills the objective back aperture, thus adding more (relatively) high-power rays at large angles. Alternately one can remove the central high-intensity rays, which contribute more to the scattering force, by changing the beam profile, either with higher order laser modes such as the $\mathrm{TEM}_{01}$ "donut" mode [33] or by blocking out the beam center with an obstruction or phase mask [34].

In the opposite (Rayleigh) regime, where the radius $r$ of the trapped sphere is $\ll \lambda$, one can again decompose the force into a scattering and gradient component. In this case the dielectric sphere is treated as an induced point dipole, and the scattering force is given by

$$
\mathbf{F}_{\mathrm{scat}}=n_{m} \frac{\langle\mathbf{S}\rangle \sigma}{c}
$$

where

$$
\sigma=\frac{8}{3} \pi(k r)^{4} r^{2}\left(\frac{m^{2}-1}{m^{2}+2}\right)^{2}
$$

is the scattering cross section of a Rayleigh sphere with radius $r,\langle\mathbf{S}\rangle$ is the timeaveraged Poynting vector, $n$ is the refractive index of the particle, $n_{m}$ is the refractive index of the medium, $k=2 \pi n_{m} / \lambda$ is the wave number of the incident light, and $m=n / n_{m}$ is the relative index. The scattering force then, as in the Mie regime, points along the direction of the light propagation and is proportional to the energy flux. The gradient force is the Lorentz force acting on the induced dipole, given by 


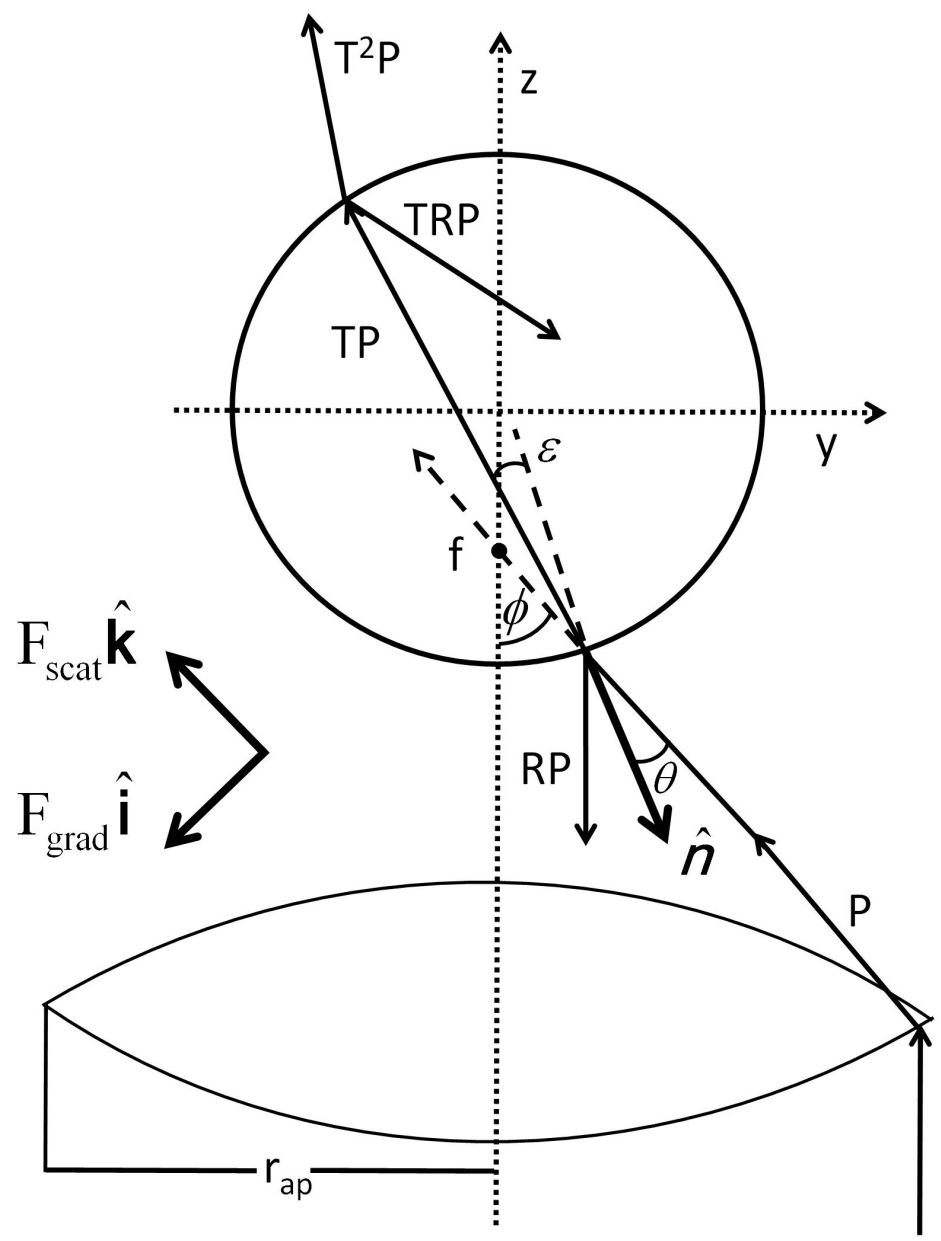

Figure 2.2: Diagram of single ray reflection and refraction in the geometrical optics regime. A ray of power $\mathrm{P}$ is directed towards a point $\mathrm{f}$ by a high NA lens, but undergoes refraction when incident upon the bead, giving rise to reflected and refracted rays of power $\mathrm{RP}, \mathrm{TP}, \mathrm{T}^{2} \mathrm{P}$, TRP, etc. These rays impart a total force which is the sum of $\mathrm{F}_{\text {scat }} \hat{\mathbf{k}}$, the scattering force, and $\mathrm{F}_{\text {grad }} \hat{\mathbf{i}}$, the gradient force. Other variables: $\theta$ is the angle of incidence, $\epsilon$ is the angle of refraction, $\phi$ is the cone half-angle of the incident beam, $\mathrm{r}_{\mathrm{ap}}$ is the radius of the objective aperture, and $\hat{\mathbf{n}}$ is the surface normal unit vector. After Ref. [31]. 


$$
\mathbf{F}_{\text {grad }}=\frac{\alpha}{2} \nabla\left\langle\mathbf{E}^{2}\right\rangle
$$

where

$$
\alpha=n_{m}^{2} r^{3}\left(\frac{m^{2}-1}{m^{2}+2}\right)
$$

is the polarizability of the particle. From Equation 2.3 we see that the gradient force is, as the name suggests, parallel and proportional to the energy density gradient (assuming the refractive index of the particle is higher than the surrounding medium, i.e. $m>1$ ). Again stable trapping is achieved by maximizing the gradient force in relation to the scattering force, which means increasing the polarizability of the particle or increasing the energy gradient. Increasing the NA of the lens used to focus the light is one way to do this, as it decreases the focal volume, which increases the gradient strength. From both the Mie and Rayleigh approximations it is clear that the NA of the lens used to focus the beam is a key determinant of the strength of the trapping force. Fortuitously for the microscopist eager to yank and tug on things in the micro-world, microscope objectives provide just the right range of NAs to stably trap micron-sized particles.

The experimental reality, however, deviates significantly from both of these theoretical limits, as typically beads with diameters on the order of a micron are trapped with $\lambda=1.064 \mu \mathrm{m}$ light, so $r \approx \lambda$. In this intermediate case, diffraction effects can not be neglected and more work is required to accurately model the momentum transfer from the laser light to the sphere. Generally, the time-averaged force for an arbitrary particle in an arbitrary electric field is given by

$$
\mathbf{F}_{i}=\left\langle\oint_{S} \mathbf{T}_{i j} \mathbf{n}_{j} d a\right\rangle
$$

where $\mathbf{T}_{i j}$ is the Maxwell stress tensor, $\mathbf{n}_{j}$ is the outward normal unit vector, the brackets denote a time average, and the integral is performed over a surface enclosing the particle. The main difficulty in calculating the above integral is deriving the six components of the electromagnetic field at the surface of the particle, as they are dependent on the scattered and internal fields in the bead, as well as the incident beam. Quantitative calculational tools using the full Lorenz-Mie theory that agree with experiments have only come of age in the last 10 years, the details of which can be found in Refs. [35, 36, 37, 38].

\section{Fluorescence Microscopy}

Fluorescence is the process by which a molecule absorbs a photon and then emits a longer wavelength photon after a certain interval, usually on the order of nanosec- 
onds. Fluorescence was first discovered in the 19th century by Herschel in quinine, the ever-popular anti-malarial-cum-gin and tonic additive, and it was further studied by Stokes, who found that the emitted photon was of longer wavelength than the absorbed photon [39]. The emission is a result of the radiative decay of electrons excited to higher energy molecular orbitals back to the ground state. The red shift (aka Stokes shift) of the emitted photon is due to the loss of the excited state's energy through other, non-radiative channels. The excitation and subsequent emission process is usually pictured in terms of a Jablonski diagram, shown in Figure 2.3. The absorption event and the various relaxation pathways have different typical timescales, generally separated by one or more orders of magnitude [40]. Absorption of the incoming photon happens on time scales in the femtosecond range, followed by a process known as internal conversion, in which the initial excited electronic state relaxes to the lowest energy level of the excited state. The energy dissipates into vibrational modes (i.e. phonons) of the molecule within picoseconds [41]. The molecule can then, depending on its solvent environment, undergo solvent relaxation, in which solvent shell molecules reorient their dipoles parallel to that of the excited state, which further lowers the energy and subsequent Stokes shift of fluorescence emission. This happens on time scales of 10-100 picoseconds. Finally, in nanosecond time frames, molecules can relax radiatively through fluorescence emission, or undergo intersystem crossing, in which the excited electron undergoes a spin flip to a long-lived triple state via spin-orbital coupling. As transition from the triplet state to the ground state is forbidden by the Pauli exclusion principle, molecules that undergo intersystem crossing stay in that state much longer $(\sim \mathrm{ms})$, finally undergoing relaxation to the ground state non-radiatively (i.e. thermally) or via a much-delayed radiative decay, termed phosphorescence.

The Stokes red shift is the aspect of fluorescence that makes it so powerful; since the emitted photons are of a different wavelength than the absorbed photons, they can be separated out from the incident light through spectral filtering. As mentioned above, the red shift is due to leakage of the initial excitation energy into other, nonradiative relaxation pathways. Additionally, there is a spectrum of energies in the ground state of large polyatomic molecules, and the molecule may relax first to a higher ground state energy before eventually decaying to the lowest one. The possibility for the fluorescing molecule to transition to a range of energy levels is illustrated in Figure 2.3 by different colored arrows. It is this range of transitions, coupled with non-radiative energy transfer to the solvent or intramolecular degrees of freedom, which give a spectrum of emitted light rather than sharp lines. A similar argument holds for the existence of an absorption spectrum rather than sharp absorption lines for large molecules in a solvent. Figure 2.4 shows an example absorption/emission spectrum for Alexa 488. The peak separation allows filtering of the fluorescence signal from the illumination light via dichroic mirrors, which reflect or pass narrow spectral bandwidths, followed by narrow-band optical filters. 


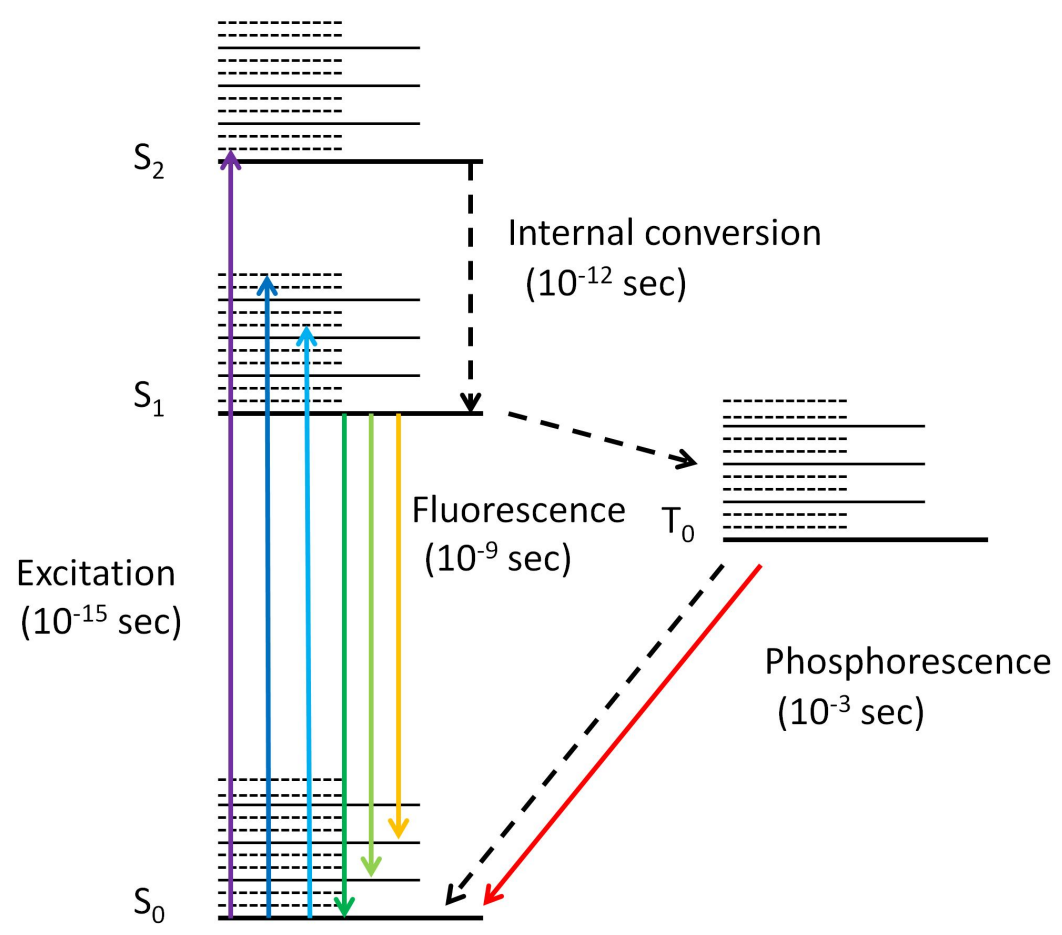

Figure 2.3: Jablonski diagram of fluorescence emission. $\mathrm{S}_{0}-\mathrm{S}_{2}$ are the ground, first, and second excited states, while $\mathrm{T}_{0}$ is the lowest energy triplet state. The thick solid black lines represent electronic energy states, the thinner solid lines the vibrational states, and the dashed lines rotational energy states. Colored arrows pointing upwards indicate photon absorption while colored arrows pointing downward represent fluorescence emission (or phosphorescence, in the case of the red arrow). Dashed arrows indicate radiationless transitions between energy levels. After [?].

The two main advantages of fluorescence over other microscopy techniques are its high contrast and the specificity with which fluorescent markers can be targeted to biological objects of interest. The high contrast allows one to detect the presence of subdiffraction limit objects, such as macromolecules, as long as they are spaced sufficiently far apart (i.e. at distances greater than the microscope's lateral resolution). It also allows for superior tracking and easier feature extraction, due to the low background. Additionally, one can target specific proteins of interest using antibody conjugated fluorophores, or by genetically tagging them with fluorescent proteins. Since the discovery and development of green fluorescent protein (GFP), fluorescent molecule tracking has become the technique of choice in biology to study dynamics.

Despite the benefits, there are of course downsides to fluorescence-based mi- 


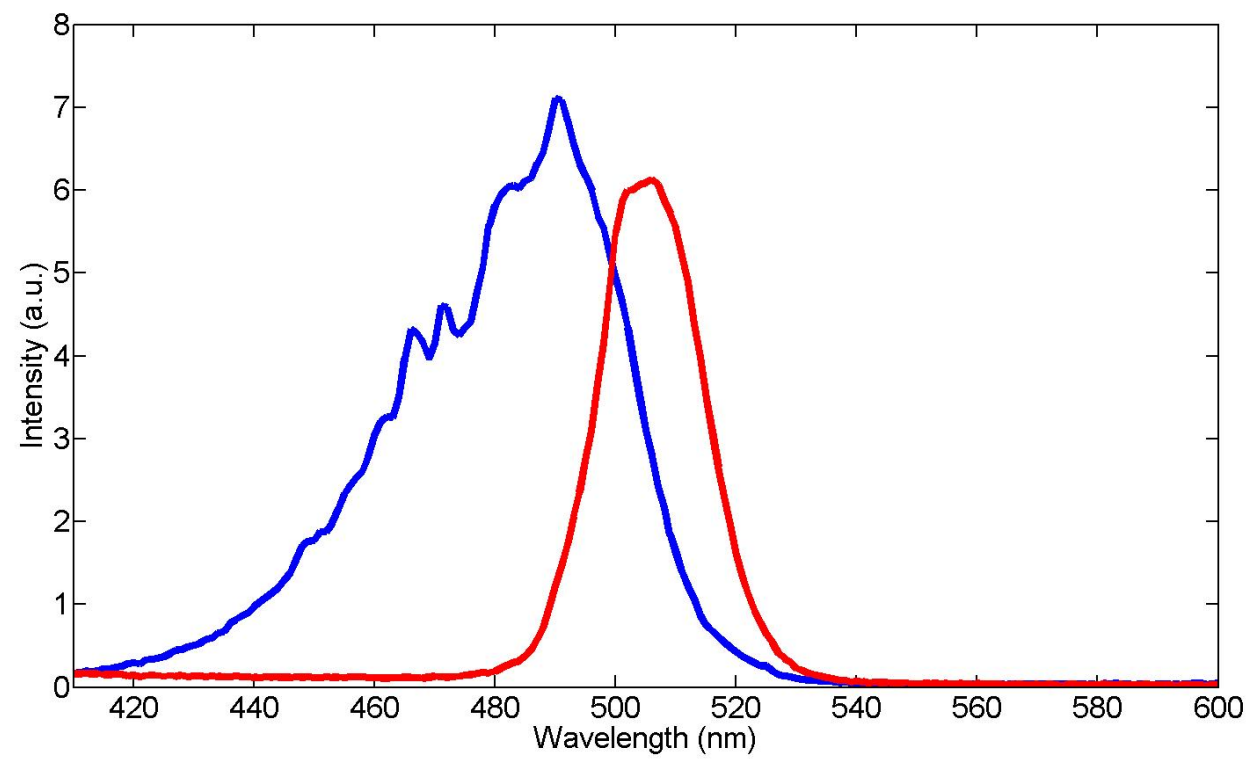

Figure 2.4: Absorption and emission profiles for Alexa 488, a dye which has an emission/absorption profile similar to GFP. Data taken by Meenakshi Prabhune.

croscopy. Fluorescence signal is inherently weak, so in order to collect enough photons to make a suitable image sometimes one needs long exposure times. This slows down imaging and can be a limiting factor when observing fast dynamics. Secondly, fluorescent proteins permanently degrade over time when excited by light, a process known as photobleaching, causing the signal to become dimmer and eventually fade out over the course of imaging. This can limit the length of time one can visualize a fluorescent object, sometimes severely so. Despite these drawbacks, fluorescence continues to be the favored imaging technique in modern biological light microscopy.

\section{Instrumentation}

Conceptually speaking, introducing an optical trap into a microscope optical path is straightforward. One only needs to insert appropriate dichroic mirrors, mirrors that selectively transmit/reflect certain wavelengths, to couple the trapping light into/out of the beam path, and appropriate photodetectors (e.g. quadrant photodiodes) to detect displacements in the trap. Moving the trap is accomplished either by keeping the trap position fixed and moving the stage or by translating/rotating the trapping beam itself. The first variant can be done with high precision by using linear piezo actuators while the second is often accomplished by converting angular beam rotations in the back focal plane (BFP) into translations in the focal plane 


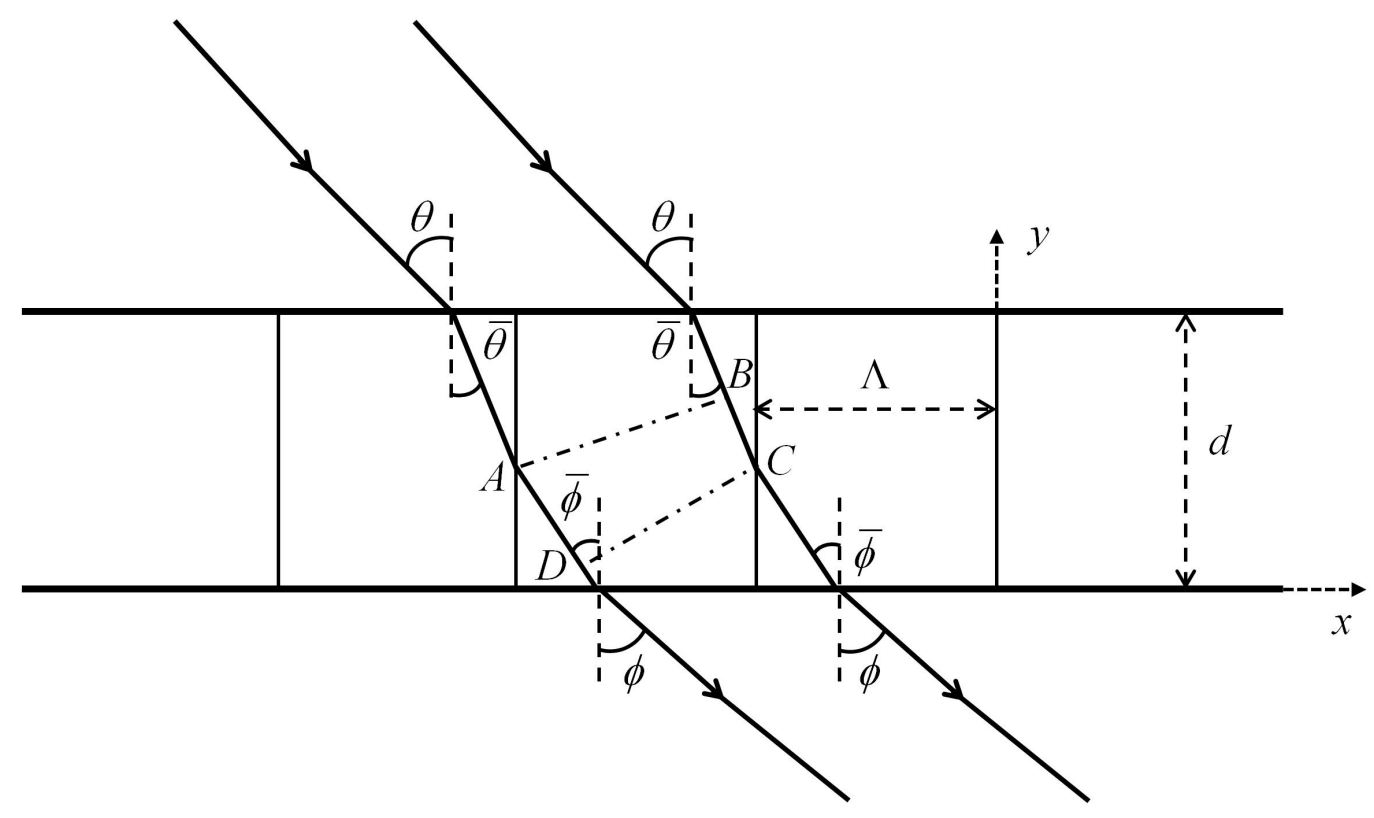

Figure 2.5: Diagram of an ultrasonic wave diffracting light. The wave travels in the $x$ direction in a region bounded by two planes a distance $d$ apart with a wavelength of $\Lambda$, which sets the distance between the planes of maximum density (solid black lines parallel to the $y$-axis). Light of wavelength $\bar{\lambda}$ in the medium is incident at an angle of $\theta$ and makes an angle of $\bar{\theta}$ with respect to the $y$-axis after it enters the region perturbed by the ultrasonic wave. After diffracting at the plane of maximum intensity, the wave makes an angle of $\bar{\phi}$ with the $y$-axis and then an angle of $\phi$ with it after leaving the perturbed region. The line segments $A B$ and $C D$ represent portions of wavefronts from the refracted and diffracted waves, respectively. After Ref. [42].

with an acousto-optic deflector (AOD) or mirror galvanometer. In our instrument we create trap translations in the focal plane using an AOD while we make axial trap translations using a piezo actuator attached to the microscope objective. Below we present an overview of the workings of AODs, piezo elements, and quadrant photodiode (QPD) detection.

\section{Acousto-optic deflection: Beam steering}

Brillouin predicted in 1922 that a liquid traversed by compression waves of short wavelengths would diffract visible light, in a manner similar to a diffraction grating, 
which was subsequently experimentally confirmed in 1932 by Debye and Sears [43]. This follows from consideration of the relation of the mean polarizability, $\alpha$, to the dielectric constant, $\epsilon$, given by the (slightly rearranged) Lorentz-Lorenz formula [42]

$$
\epsilon=\frac{1+\frac{8 \pi}{3} N \alpha}{1-\frac{4 \pi}{3} N \alpha}
$$

where $N$ is the number of molecules per unit volume. Compression waves create regions of compression and rarefaction, thus modulating the number density of molecules in a given volume. From Eqn. 2.5 we see that this leads to a time and space varying dielectric constant, which is usually assumed to be of the form

$$
\epsilon=\epsilon_{0}+\epsilon_{1} \cos (K x-\Omega t)
$$

for an acoustic wave traveling in the $x$ direction, where $\epsilon_{0}$ is the average dielectric constant of the material, $\epsilon_{1}$ is a variation in the dielectric constant determined by the variation in number density, $K=2 \pi / \Lambda$ is the wave number of an acoustic wave of wavelength $\Lambda$, and $\Omega=K v$ is the angular frequency of an acoustic wave traveling at speed $v$. Since the speed of light in a medium is always much greater than the speed of sound in that medium, we can consider the variations in the medium's refractive index to be stationary to first-order. Figure 2.5 depicts the refraction of an incident plane light wave from an ultrasonic (short-wavelength) sound wave. From the theory of diffraction gratings, we can surmise that there will be maxima in the intensity for angles $\bar{\phi}$ where the optical path difference between rays scattered from two successive planes are integer multiples of $\bar{\lambda}$, so

$$
B C-A D=\Lambda\left(\sin \bar{\phi}_{l}-\sin \bar{\theta}\right)=l \bar{\lambda}
$$

given the variables definitions in Figure 2.5 and where $l=0, \pm 1, \pm 2, \ldots$ Using Equation 2.7 it can be shown that the angular separation between successive orders is given by

$$
\sin \phi_{l}-\sin \phi_{l-1} \simeq \phi_{l}-\phi_{l-1}=\frac{\lambda}{\Lambda}
$$

The preceding equation shows why one doesn't see diffraction effects for $\Lambda$ 's on a macroscopic scale: as $\Lambda$ gets larger and larger, the angular separation between diffraction peaks disappears. This of course also implies that higher frequency waves give a better spatial separation of the diffraction peaks, which is why sound waves in the ultrasonic $(\mathrm{MHz})$ range are used in acousto-optic devices.

AODs produce sound waves in paratellurite crystals $\left(\mathrm{TeO}_{2}\right)$ by oscillating an attached piezoelectric transducer. The standing compression wave creates a periodic mechanical anisotropy in the crystal refractive index which, as noted above, leads to optical anisotropy. The general phenomenon by which mechanical stresses lead 
to optical anisotropy is referred to as the photoelastic effect and was first described by Brewer in the 19th century [44]. Raman and Nath approximated this kind of diffracted optical field as (series expanding and dropping 2nd order terms) [45]

$$
\frac{d E_{m}}{d x}+\frac{\xi}{2 L}\left(E_{m+1}-E_{m-1}\right)=-i \frac{m K}{\cos \theta_{0}}\left(\sin \theta_{0}-m \sin \theta_{B}\right) E_{m}
$$

where

$$
\xi=-k_{f} \Delta n L / \cos \theta_{0}
$$

and the Bragg angle, $\theta_{B}$, in the medium is defined as

$$
\cos \theta_{B}=\frac{K}{2 k}
$$

$E_{m}$ is the amplitude of the $m$ th order diffracted light wave with frequency $\omega+m \Omega$, $\theta_{0}$ is the angle of incidence in the medium (same as $\hat{\theta}$ above), $L$ is the width of the crystal (same as $d$ above), $k_{f}$ is the wavenumber of the incident light in a vacuum, $k$ is the wavenumber in the medium, $\Delta_{n}$ is the amplitude of the change in refractive index (like $\epsilon_{1}$ above), $m \in \mathbb{Z}$, and $K$ and $\Omega$ are the wavenumber and frequency of the sound wave, as above. Though Eqn. 2.9 can't be solved analytically in general, the low and high frequency limits can be and they contain the relevant physics for our AOD. It is standard practice to define a factor, $Q=2 K L \sin \theta_{B} / \sin \theta_{0}$, whose dependence on acoustic wavelength and incidence angle make the high and low frequency limits of Eqn. 2.9 more explicit [45].

In the low frequency limit, $Q \ll 1$, we can neglect the $m \sin \theta_{B}$ contribution to Eqn. 2.9. It then becomes solvable, and the solution for the amplitude of light in the $m$-th diffracted order is given by the product of an $m$-th order Bessel function and an exponential phase term. This gives so-called Raman-Nath diffraction, in which the light intensity is partitioned roughly equally among the various $m$-order diffracted beams, given that the acoustic beam width $L$ is not too large in comparison with the other length scales and that the beam incidence is normal or very nearly so. Though this diffraction is not useful for beam steering, the Raman-Nath diffraction pattern can be useful in initial AOD alignment with the beam path, as it i) tells the user that the AOD is functioning, and ii) that the beam is incident normal to the AOD crystal surface.

In the high frequency limit, $Q \gg 1$, if $\theta_{0} \approx \pm \theta_{B}$, then Eqn. 2.9 again simplifies and the 0 th and 1 st order terms predominate. If $\theta_{0}$ is identical to $\theta_{B}$ then the Bragg condition is met, and the (normalized) intensity in the first-order diffracted beam is given by [45]

$$
I_{1}=\sin ^{2}(\xi / 2)
$$


Theoretically all of the light intensity could be channeled into the first diffracted order if $\xi=\pi$, or some odd multiple thereof. In practice though, the crystal size, which constrains the acoustic beam width $L$, is the limiting factor. In modern AODs typically $70-80 \%$ of the beam power can be channeled into the first-order beam. By modulating the driving frequency of the piezo transducer one can change the sound wave frequency, which changes the Bragg angle and angularly rotates the beam. Typically commercial AODs can rotate the beam on the order of tens of milliradians, which generates translations on the order of tens of micrometers given typical microscope optics.

\section{Piezo actuators: Axial motion}

Piezoelectric materials are materials which produce a voltage under mechanical pressure, or conversely, generate a force (i.e. expand) in the presence of an applied electric field. This effect is reliant on the presence of permanent electric dipoles in the material, and is exhibited by a wide range of different materials, from non-inversion symmetric crystals, to ceramics, to biopolymers like DNA and collagen. A related effect, pyroelectricity, was first observed by sailors in the 18th century when they noted that the tourmaline they brought back from the Orient could attract hot ashes. This was shown to be due to the production of opposite charges at opposite ends of the crystal, and the Curie brothers demonstrated that a similar effect could be induced by exerting pressure on certain crystals [46]. In so-called ferromagnetic materials the spontaneous dipole alignment underlying this effect can be reversed by the application of an external electric field, and it is these materials that are used in commercial piezo actuators.

The most commonly used piezoelectric material is a ferroelectric ceramic called lead zirconate titanate (PZT). A PZT unit cell is shown in Figure 2.6. Below the Curie temperature $\left(\sim 230 \mathrm{C}^{\circ}\right)$ the unit cell is asymmetric, exhibiting a spontaneous electrical polarization, while above the Curie temperature the unit cell becomes symmetric and loses its piezoelectric properties. The crystallites which make up the ceramic are randomly ordered, and so there is no macroscopic ferroelectricity when the ceramic is sintered into a solid. Macroscopic alignment of the crystal domains is done by applying a large static electric field at temperatures near the Curie temperature, where the electric permittivity takes on large values, in a process known as "poling" [47]. After poling the ceramic has macroscopic ferroelectric properties, and mechanical strains can be induced by applying a voltage across the material. In practice the strains are quite small and stacks of parallel-aligned piezo ceramics are used to generate larger displacements. Poled ceramics are only truly piezoelectric, i.e. linear, in a certain range of electric fields (typically up to $500 \mathrm{~V} / \mathrm{cm}$ ). At higher fields the polarization of the crystal domains is enhanced and the strain scales with the square of the field strength, leading to hysteresis. In general the electrical and 

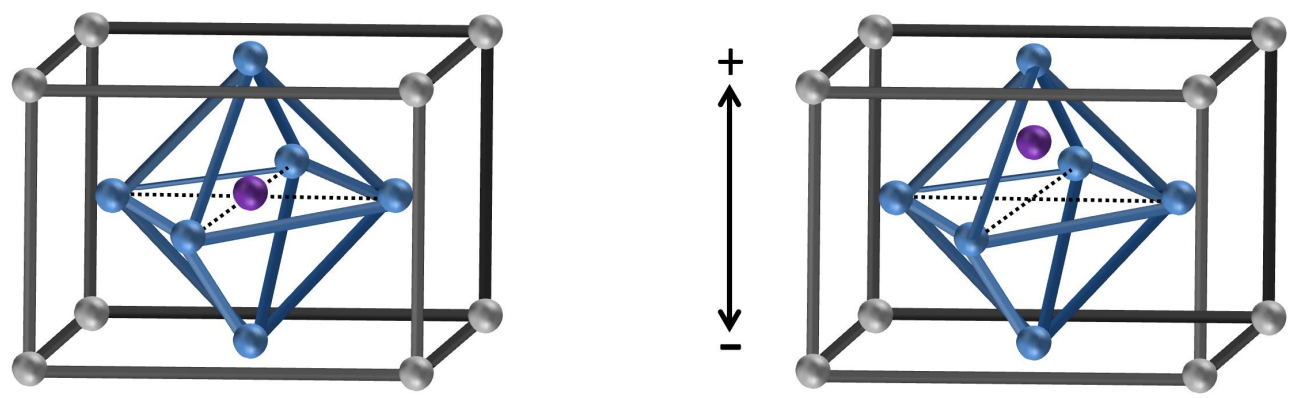

Figure 2.6: Drawing of a PZT unit cell. Blue spheres represent $\mathrm{O}^{2-}$ ions, gray spheres represent lead atoms, and the central purple sphere is a zirconium or titanium ion. On the left is a unit cell above the Curie temperature, which is inversion symmetric and has no inherent dipole moment. On the right is a unit cell below the Curie temperature, where the central ion is displaced from the mid plane which breaks symmetry and leads to a spontaneous dipole moment. After Ref. [47].

elastic fields in the material are related via the tensorial piezoelectric equations of state, but the change in length, $\Delta L$, of a single piezo actuator in the low $E$-field limit can be estimated as [47].

$$
\Delta L=S L_{0} \approx \pm E d_{i j} L_{0}
$$

where $S$ is the strain, $L_{0}$ is the initial length, $E$ is the magnitude of the applied electric field, and $d_{i j}$ is the piezoelectric coefficient of the material along the $i j$ material axis. Along the axis of polarization the typical piezoelectric coefficient for a linear actuator from our manufacturer is $250-500 \mathrm{pm} / \mathrm{V}$, so the displacements induced can be on the order of nanometers for typical stack sizes and electric field strengths. This is how piezoelectrics earn their keep in biophysics; they allow precise positioning in the nanometer-regime but can have total travel ranges on the order of hundreds of micrometers, thus spanning the relevant length scales for most applications.

\section{Quadrant photodiodes: Displacement detection}

Optical trapping provides a powerful way to manipulate microscopic objects but turning it into a quantitative tool requires a knowledge of the forces exerted by the trap and the displacements of the trapped object. As discussed previously, deriving the force exerted by the trap on a bead of the size typically used in experiments is challenging and still inaccurate when variables such as bead size, surface roughness, and deviations from spherical geometry are unknown and not taken into account. 


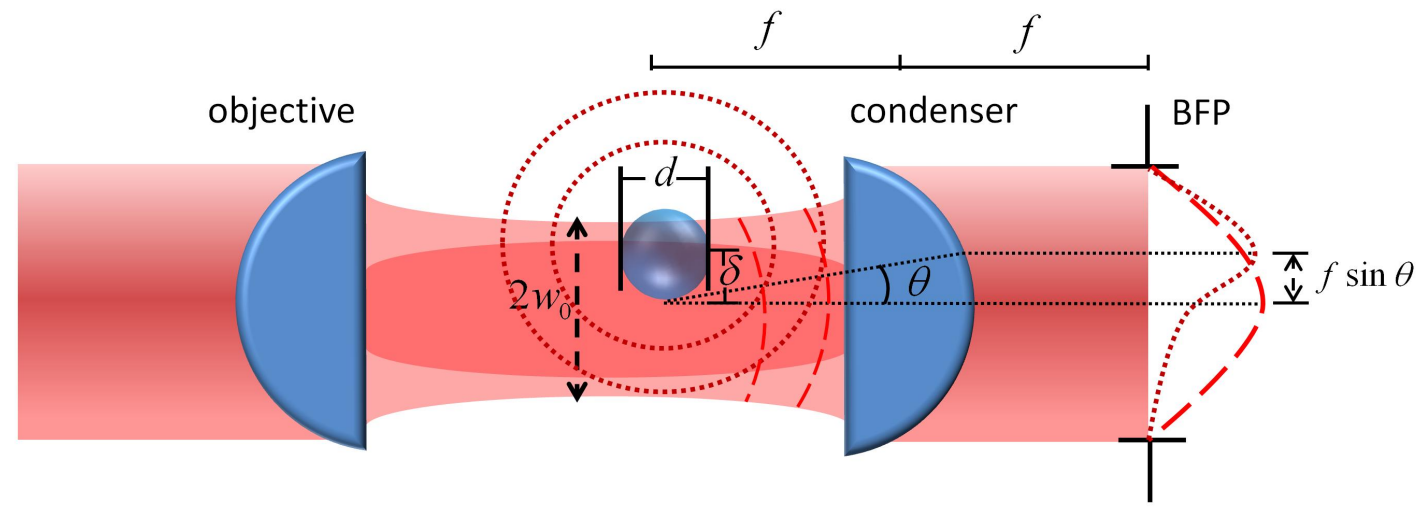

Figure 2.7: Drawing of forward scattered detection (FSD) in an optical trap. An optically trapped bead is displaced a distance $\delta$ from its equilibrium trapping position in a beam of focal waist radius $w_{0}$, shifting the interference peak by a distance $f \sin \theta$. Here $d$ gives the diameter of the bead, $f$ is the focal length of the condenser, $\theta$ is the angle an outgoing ray makes with the optical axis. The dotted red lines represent wavefronts scattered from the bead and the dashed red lines are the unscattered wavefronts, which interfere in the BFP according to the bead position in the trap. After Ref. [48].

Practically this makes force determination a measurement question, one which is addressed by detecting the light scattered from the sphere in the far-field with a photodiode. In most applications the experimentalist is interested in measuring forces exerted on the bead by an attached object, so the detector signal must be calibrated to reflect meaningful units.

Typically displacement signals are measured via laser interferometry in a plane conjugate to the back-focal plane (BFP) of the condenser, as shown in Figure 2.7. This type of displacement detection is known as forward-scattered detection (FSD), and it relies on interference between the scattered and unscattered beam components. In the approximation of a Rayleigh scatterer and a paraxial, scalar Gaussian beam, the outgoing unscattered electric field can be approximated as [49]

$$
E(\mathbf{r}) \approx \frac{-i k w_{0} I_{\mathrm{tot}}^{1 / 2}}{r\left(\pi \epsilon_{s} c_{s}\right)^{1 / 2}} \exp \left(i k r-k^{2} w_{0}^{2} \theta^{2} / 4\right)
$$

and the outgoing scattered field can be approximated by

$$
E^{\prime}(\mathbf{r}) \approx \frac{k^{2} \alpha}{r} E(x) \exp (i k(r-x \sin \theta \cos \phi))
$$

where 


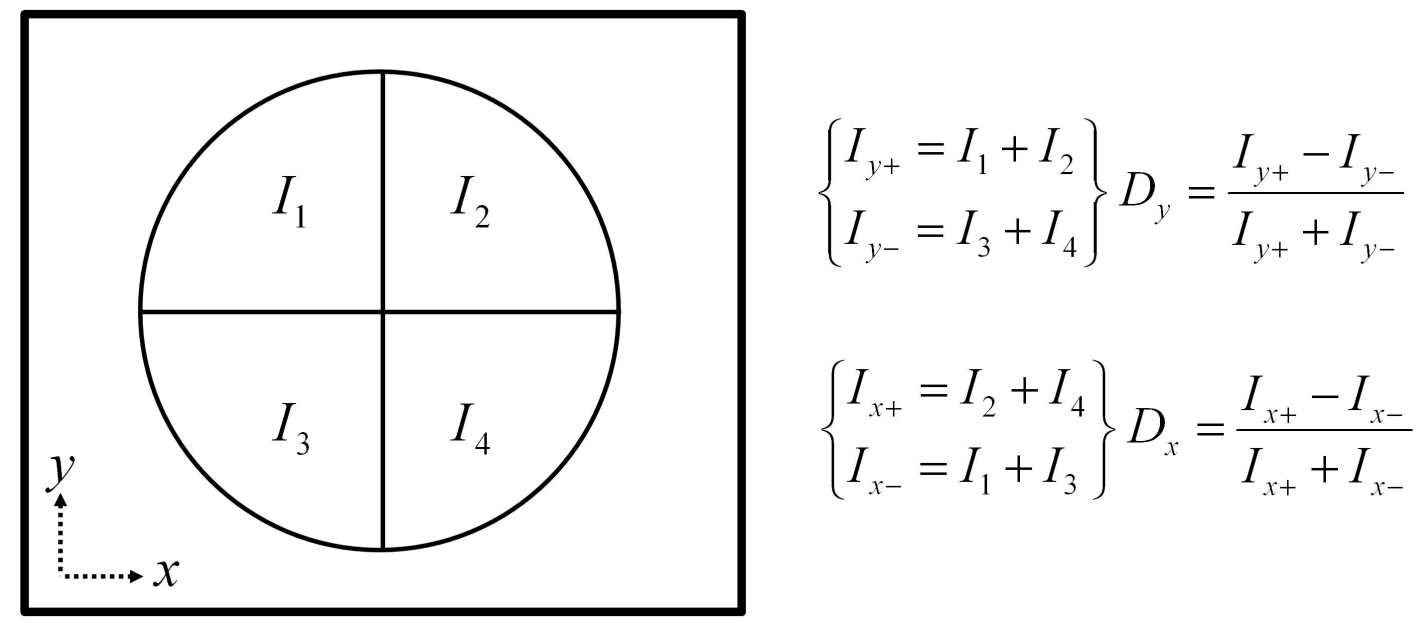

Figure 2.8: Schematic of intensity detection with a QPD. The diode is split into four quadrants and the $x$ and $y$ components of the intensity distribution are calculated as shown on the right. $I_{1-4}$ are the intensities in quadrants $1-4$ and $D_{x} / D_{y}$ give the intensity changes along the $x / y$ coordinate. After Ref. [48].

$$
E(x)=\frac{2 I_{\text {tot }}^{1 / 2}}{w_{0}\left(\pi \epsilon_{s} c_{s}\right)^{1 / 2}} \exp \left(-x^{2} / w_{0}^{2}\right)
$$

is the unscattered field at the point in the focal plane where the scattering particle is located. It is assumed for simplicity that the particle is only displaced in the $x$ direction. In the above equations, the litany of variables is as follows; $k$ is the incident light's wavenumber in the solvent, $w_{0}$ is radius of the beam waist at the focus $(1 / e), \theta$ is the angle made by the outgoing ray with the optical axis (as in Fig. 2.7), $\phi$ is the azimuthal angle (angular rotation around the focal axis), $I_{\text {tot }}$ is the total intensity, $\epsilon_{s}$ and $c_{s}$ are the permittivity and speed of light in the solvent, $\alpha$ is the polarizability of the scattering particle, and the phase factor $e^{-i \omega t}$ is implicit. The factor of $-i$ in front of Eqn. 2.12 is due to the Gouy phase shift that the unscattered light picks up as it goes through the focus. This is important for the far field interference between the two beams, and adds a relative phase shift of $\pi / 2$ between them. By taking into account only first-order interference effects in the farfield limit, the authors of Ref. [49] were able to derive an expression for the angular interference pattern due to a particle in the focal plane displaced a distance $x$ from the optical axis.

More important than the general angular interference pattern, though, is the shift of the intensity center-of-mass in the BFP for a given displacement, as this 


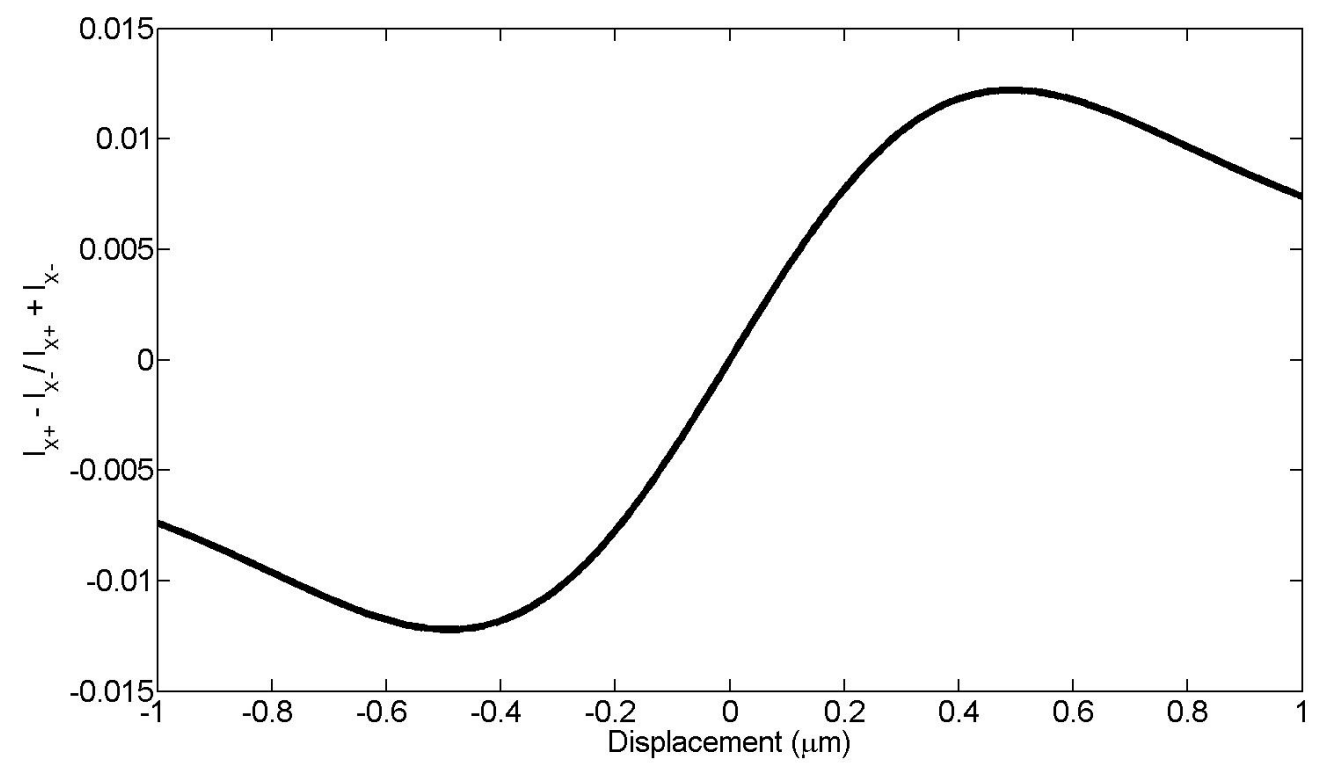

Figure 2.9: Plot of theoretical detector response from Eqn. 2.14. This plot assumes a $0.5 \mu \mathrm{m}$ glass bead in water with a beam waist radius of $w_{0}=0.53 \mu \mathrm{m}$ and $1064 \mathrm{~nm}$ laser light, as per Ref. [49].

can be measured with a photodiode. For a quadrant photodiode (QPD), pictured in Figure 2.8, the intensity change due to a displacement, $x$, of the trapped bead is given by [49]

$$
\frac{I_{x+}-I_{x-}}{I_{x+}+I_{x-}} \approx \frac{16}{\sqrt{\pi}} \frac{k \alpha}{w_{0}^{2}} G\left(x / w_{0}\right)
$$

where

$$
G(u)=e^{-2 u^{2}} \int_{0}^{u} e^{y^{2}} \mathrm{~d} y
$$

is the Dawson integral. Figure 2.9 shows a plot of theoretical detector response vs. displacement for variable values similar to those in Ref. [49]. The detector response is relatively linear for displacements of $\sim \pm 200 \mathrm{~nm}$, and then starts to rapidly deviate from linearity for increasing displacements. This linear regime is where detection is generally done, as one can straightforwardly relate the photodiode signal to the bead displacement. While the expressions for the intensity changes derived above are calculated in the Rayleigh limit, they turn out to be surprisingly robust, and the detector response curves for larger beads are qualitatively the same.

Though FSD is the most common detection modality for optical trapping, one can also use the light scattered back into the objective to track bead displacements, 
a technique known as back-scattered detection (BSD). This technique has the advantage of simplicity, e.g. the "condenser" is always in perfect alignment, but the disadvantage that the signal is 10-100 times weaker than in FSD. Since our microscope is designed to be used with opaque samples, we have no condenser and so are confined to using BSD for displacement detection.

There are a some qualitative differences between the two detection types, though in practice the detection and calibration techniques are the same. In contrast to the interferometry technique used in FSD, in BSD one detects back-scattered signals in some intermediate plane between the BFP and the image plane, typically closer to the latter. Choosing this plane is generally done by trial and error, where one searches for a position that has a good signal-to-noise ratio (SNR) and where the reflected light spot size isn't too concentrated (filling up maybe $1 / 3$ to $1 / 2$ of the QPD detection zone). BSD is also quite sensitive to reflections from other surfaces, which reduce dynamic range and can worsen SNR, so one also endeavors to find a plane where additional reflections don't impinge on the QPD surface or can be spatially filtered out. Reference [50] gives a systematic comparison of FSD and BSD. One interesting point that comes out of their analysis is that position sensitive detectors (PSDs), photodetectors that sense the center of mass of a spot of light by measuring resistive changes, have a slightly greater range of linear response than QPDs in BSD. Although the interference patterns in the detection plane are dissimilar for the two techniques, the resultant detector response curves have the same shape (as in Fig. 2.9) [50, 51].

Detection in three dimensions can be achieved with either technique, with the axial position being given by the sum signal incident on the QPD. In FSD the axial signal is derived from the total intensity of the interference pattern in the BFP (the sum of all the QPD quadrants), which is a function of the bead's axial position in the trap. This intensity dependence is due to the dependence of the relative phase between scattered and unscattered electric fields on the axial position, as derived in exhaustive detail in Reference [35]. They find that the total intensity in the BFP of the condenser, $I_{\text {sum }}(\Delta z)$, due to the intensity of two waves of (complex) amplitudes $\mathbf{E}_{0} A_{1}$ and $\mathbf{E}_{0} A_{2}$ separated by a distance $\Delta z$ (scattered and unscattered wave) is given by

$$
I_{\text {sum }}(\Delta z)=\epsilon_{0} c \frac{E_{0}^{2} k_{n}^{2}}{(2 \pi)^{2}}\left|A_{1} k_{0}+\frac{i A_{2}}{\Delta z}\left[\exp \left(-i \Delta z k_{0}\right)-1\right]\right|^{2}
$$

where $\epsilon_{0}$ is the vacuum electric permittivity, $k_{n}$ and $k_{0}$ are the wavenumbers of light in the dielectric medium and vacuum, respectively, $E_{0}$ is the amplitude of the electric field incident on the objective BFP, and $c$ is the speed of light. The $z$ dependence in the 2nd term of the above equation encodes the Gouy phase shift of light going through a focus (see Fig. 2.10). The theoretical detector response given by plotting Eqn. 2.15 looks like the response shown in Fig. 2.9, except with the peaks 


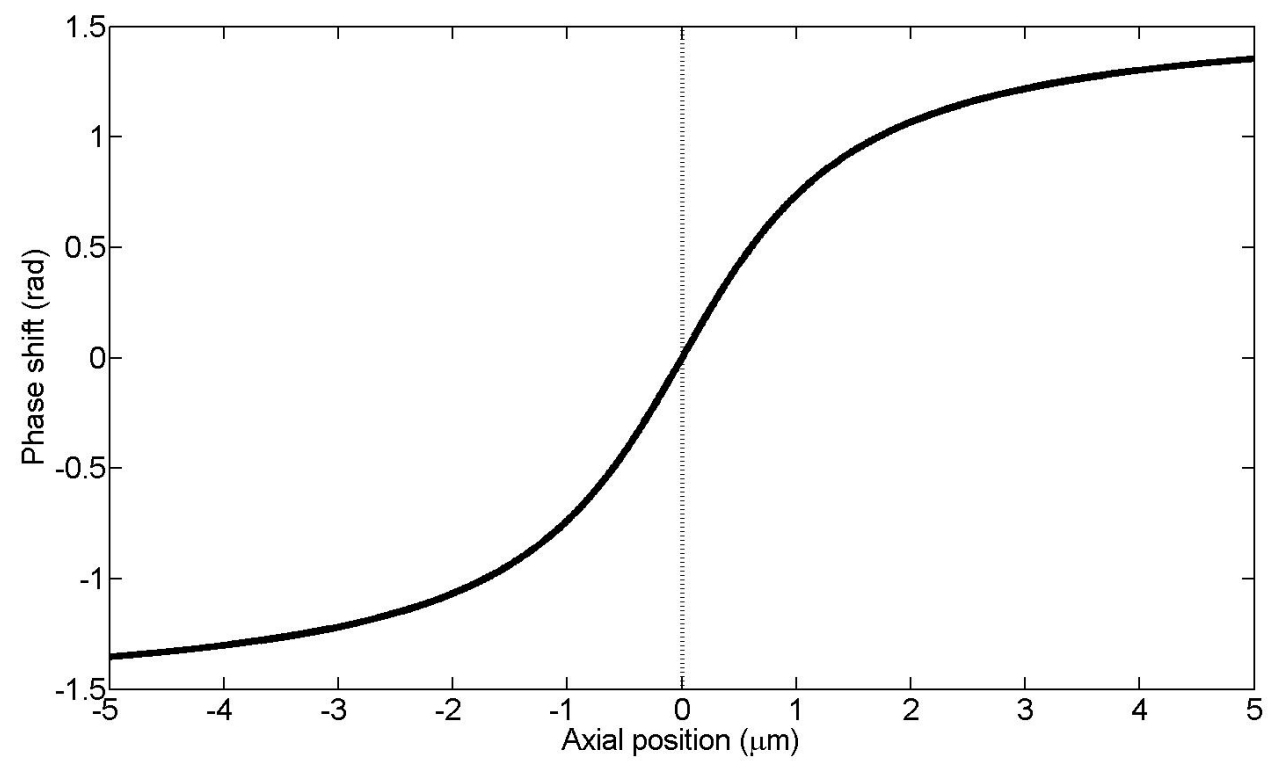

Figure 2.10: Calculation of the Guoy phase shift for a Gaussian beam of $1064 \mathrm{~nm}$ light in water. The phase shift is given by $\Phi=\arctan \left(\lambda z / \pi w_{0}^{2}\right)$ [55] and here we assume a beam waist radius of $w_{0}=0.53 \mu \mathrm{m}$ and $\lambda=\lambda_{0} / n_{\text {water }}=0.8 \mu \mathrm{m}$.

inverted (i.e. $f(x) \rightarrow-f(x)$ ). Various authors $[52,53,54]$ have shown experimental detector response curves similar to those given by Eqn. 2.15 and have developed various methods to calibrate axial detection. It's worth mentioning that the axial detection sensitivity changes non-monotonically with the condenser numerical aperture, achieving a maximum for NAs of around 1.1, while lateral detection sensitivity increases with increasing numerical aperture $[52,54]$. Non-monotonicity of the axial detection sensitivity is due to the fact that the intensity in some regions of the BFP are positively correlated with the axial position (the inner region of the detector) while others are negatively correlated with it (outer regions), so if one integrates over the entire angular range some portions cancel out, leading to loss of sensitivity.

Backscattered axial detection is also possible, but since only scattered light is collected for detection the phase relationships differ from those mentioned above. To our knowledge there have been no rigorous theoretical treatments of BSD. Presumably intensity variations in the BSD axial signal come primarily from reflections from the bead's two interfaces, and interference effects in the BSD signal could depend on this spacing. It has been noted that an axially trapped bead's equilibrium position varies periodically with the bead size, with diameters corresponding to halfwavelength multiples of the incident light sitting closer to the focus of the trap [56]. This effect is thought to arise from destructive interference of standing waves in the 


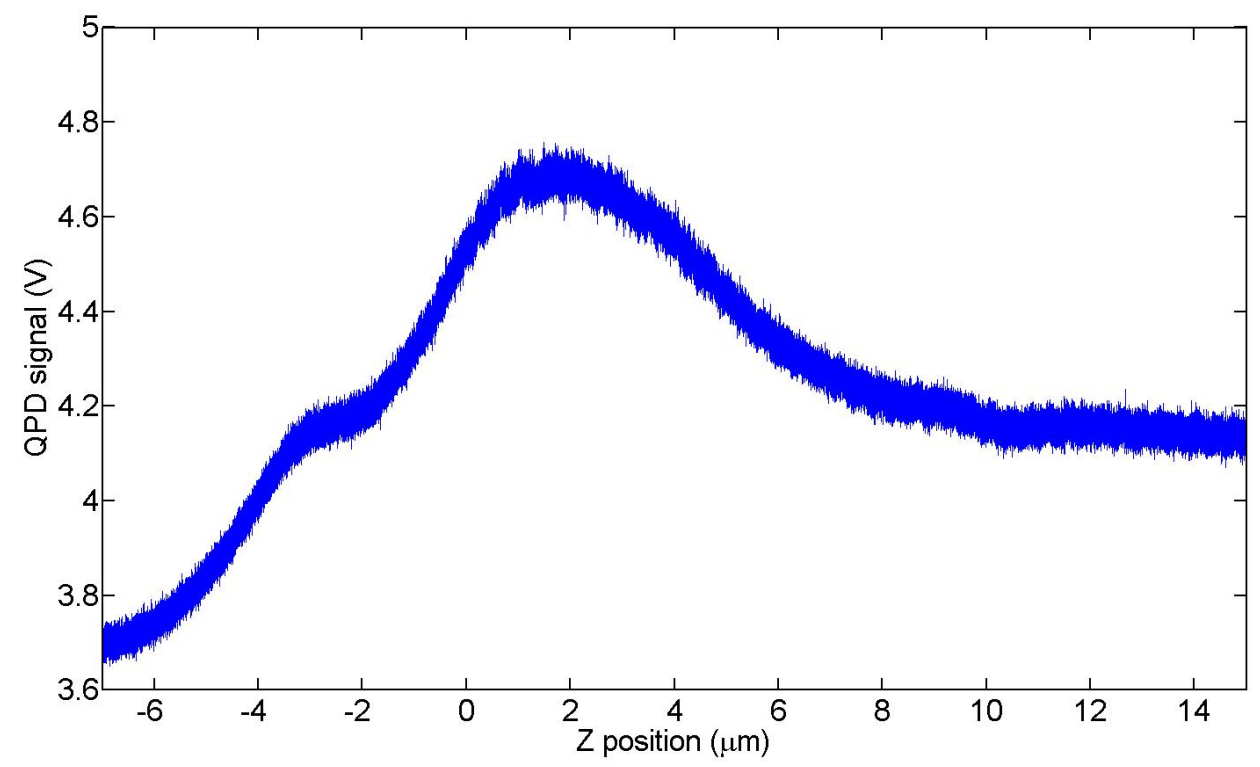

Figure 2.11: QPD response in BSD for an axial scan of a $3 \mu \mathrm{m}$ silica bead attached to a coverslip. The scan was performed by stepping the objective axially in $100 \mathrm{~nm}$ increments with a piezo actuator.

bead, causing beads of certain diameters to act as if they were anti-reflection coated. The flip side of this is that they scatter very little light backwards and are difficult to detect with BSD, making bead diameter a relevant parameter when trying to maximize the weak BSD signal. The axial signal also looks qualitatively different than with FSD, as can be seen in Figure 2.11. The detector signal in Fig. 2.11 looks similar to the curves obtained in Ref. [51] for axial BSD from silicon fiducial markers. Fortuitously there is still a linear region in the photodiode response around zero which can be exploited.

To gain relevant physical information from our detector signal we must first calibrate it. There are various ways to do this, and Ref. [30] gives an excellent description of different calibration schemes. Here we will only discuss the power spectrum method, which is how we perform all of our calibrations of trap stiffness and detector sensitivity. With the assumptions that our trapped bead is spherical, is in a harmonic potential, and that its motion is thermally driven, we can write down its $1 \mathrm{D}$ equation of motion along the coordinate $x$ as

$$
\kappa x-\beta x^{\prime}=F_{t h}(t)
$$

where $\kappa$ is the trap stiffness, $\beta=6 \pi \eta r$ is the drag coefficient for a bead of radius $r$ in a medium of viscosity $\eta$, and $F_{t h}$ is a white noise force with zero mean. The inertial 


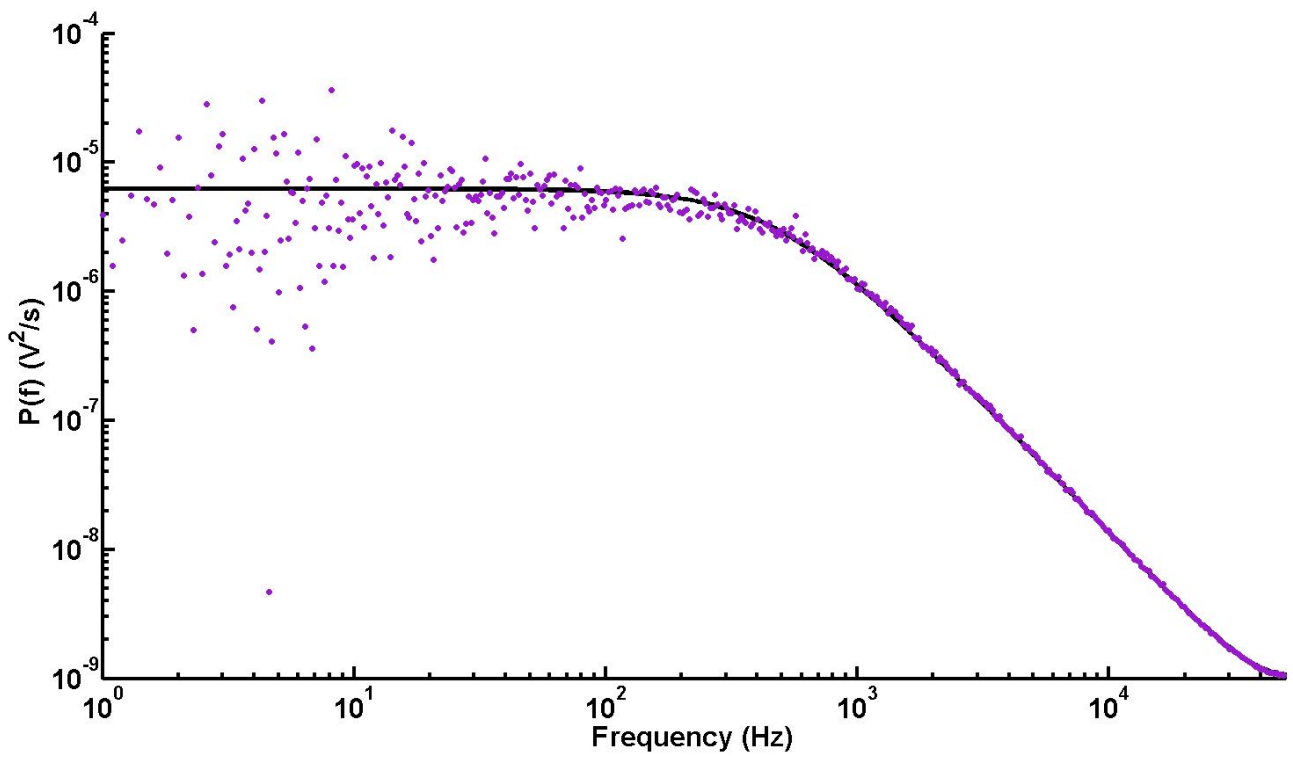

Figure 2.12: One-sided power spectrum of the lateral excursions in one dimension of a trapped $1 \mu \mathrm{m}$ silica bead. The violet dots represent block averaged, log-binned data while the solid black line is the fitted Lorentzian. At frequencies far below the roll-off frequency, the power spectrum is roughly constant, whereas at higher frequencies the bead motion looks diffusive. Fit done using the MatLab package TweezerCalib described in Ref. [57].

contribution is negligible due to the low Reynolds number of the system and it is neglected [31]. Equation 2.16 describes Brownian motion in a parabolic potential and can be solved exactly. Its dynamics are manifest in the one-sided Lorentzian power spectrum

$$
S_{x x}(f)=\frac{k_{B} T}{\pi^{2} \beta\left(f_{0}^{2}+f^{2}\right)}
$$

where $S_{x x}(f)$ is in units of displacement ${ }^{2} / \mathrm{Hz}, k_{B}$ is Boltzmann's constant, $T$ is the absolute temperature, and $f_{0}=\kappa / 2 \pi \beta$ is the corner frequency. Figure 2.12 shows a fit of Equation 2.17 to experimental data. One sees two different regimes in the plot; one for frequencies much lower than the corner frequency, $f \ll f_{0}$, and one for frequencies much higher than the corner frequency, $f \gg f_{0}$. In the low frequency regime the power spectrum is roughly constant, with a value of $S_{0}=4 \beta k_{B} T / \kappa^{2}$ (the $f=0$ value). This regime reflects the confinement of the trap, which keeps the bead in the same place over longer times (e.g. lower frequencies). Conversely, for $f \gg f_{0}$ the power spectrum falls off with the characteristic $f^{-2}$ slope of Brownian 
noise. This is a sign of the "wiggle room" that the bead has in the trap; at very short times/high frequencies, the bead looks as if it's freely diffusing in the harmonic potential, as at these time scales it hasn't run up against the "walls" of the potential well yet. Figure 2.12 is rich in data and we can fit it to obtain the corner frequency, which is related to the trap stiffness as noted above. The other relevant factor for calibration is the detector sensitivity, $\rho$, which allows one to convert the measured photodiode voltage signal into units of displacement. The photodiode voltage power spectrum, $S_{v v}$, is related to the displacement power spectrum by

$$
S_{v v}(f)=\rho^{2} S_{x x}(f)
$$

The detector sensitivity can be obtained, independent of trap stiffness, by fitting the product of the power spectrum and the frequency squared, $S_{v v} \cdot f^{2}$, which asymptotically approaches $k_{B} T / \pi^{2} \beta$ [23]. Graphing this gives a rotated Lorentzian, where the $f^{-2}$ slope becomes a plateau, from which the value can easily be read off. The sensitivity is related to the plateau value, $P^{V}$, of the rotated spectrum by

$$
\rho=\sqrt{\frac{6 \pi^{3} \eta r P^{V}}{k_{B} T}}
$$

The plateau value is of course also the slope of the line asymptotic to the power spectrum at $f \rightarrow \infty$. This method for calibration determination has been shown to agree with other more direct methods to within $\sim 20 \%$ [23]. While the power spectrum method is clearly powerful, as it gives us all the variables we need for calibration with one easily performed experimental measurement, it also has its drawbacks. One must assume the bead size, the viscosity of the solvent, the sphericity of the particle, and a harmonic trapping potential, all of which may not be the case. Practically speaking, for a well-aligned trap the harmonic potential is a good approximation, and isn't usually a large source of error. While manufacturers are good at making relatively spherical microparticles in a given size range, monodispersity is still a dream and bead size can vary up to $10 \%$. Viscosity is in general temperature dependent, and heating of the solvent in the laser focus where the bead sits is an additional source of error. In the end though, given the high error margins in most biophysical experiments, the power spectrum method of calibration is rarely the main source of error and its ease of implementation makes it one of the most used calibration techniques. 


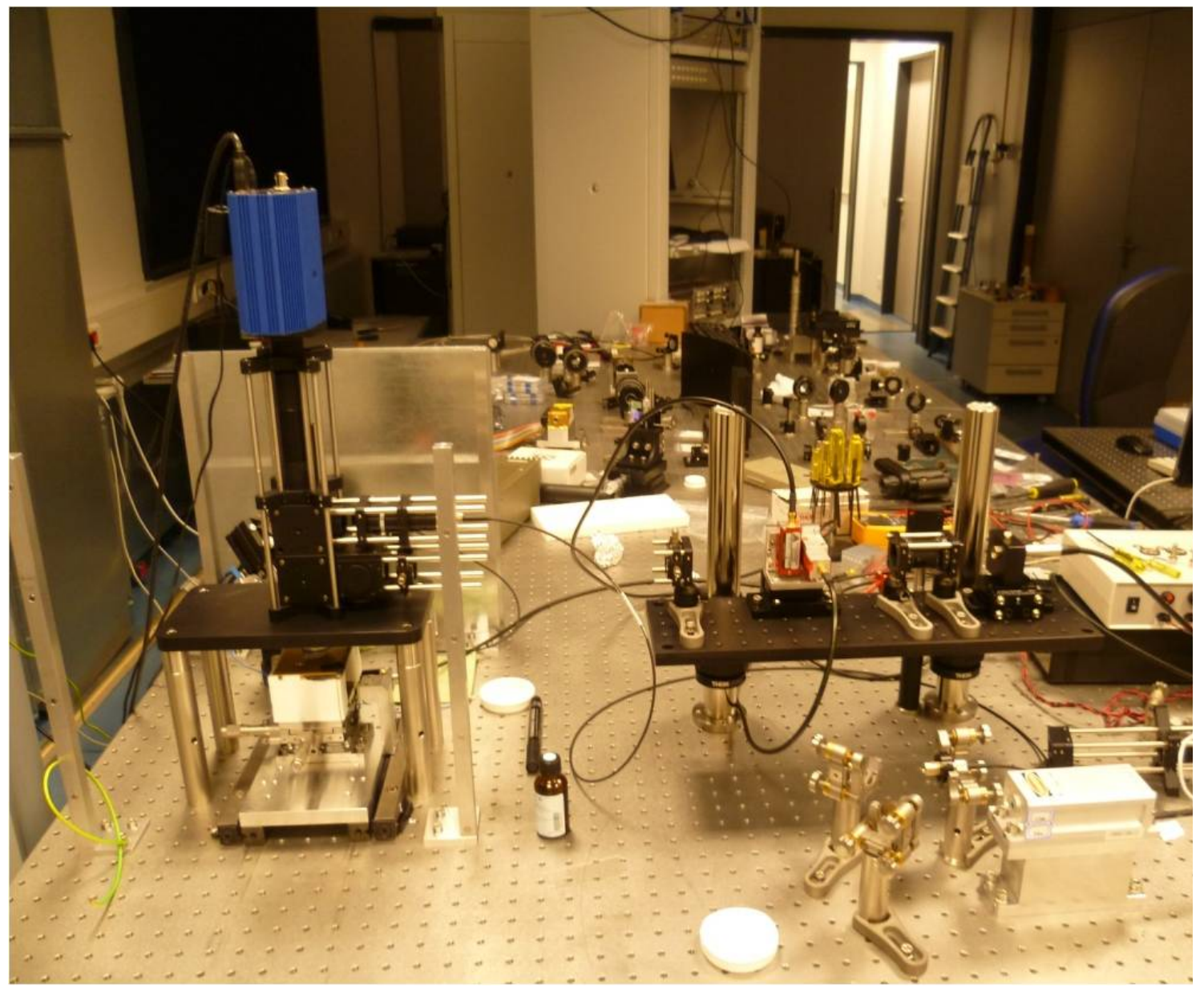

Figure 2.13: Photograph of the setup. The microscope body is on the left while the trapping laser is on a raised black platform to the right. Fluorescence illumination light from the blue laser in the right-hand foreground is coupled into the microscope via a single-mode fiber.

\section{The epifluorescence microscope/optical trap/patch clamp setup}

Figure 2.13 shows a photograph of our setup. The main microscope body is on the left while the trapping laser and associated components are on a separate raised platform on the right. The blue fluorescence laser is also on the right, mounted on an aluminum platform attached to the optical table, while the micromanipulator is behind the microscope body and obscured in the photo. Figure 2.14 shows a schematic of the setup. Starting with the trapping laser, the components in the beam line are as follows: the trapping laser is a linearly polarized ( $p$-polarized with 


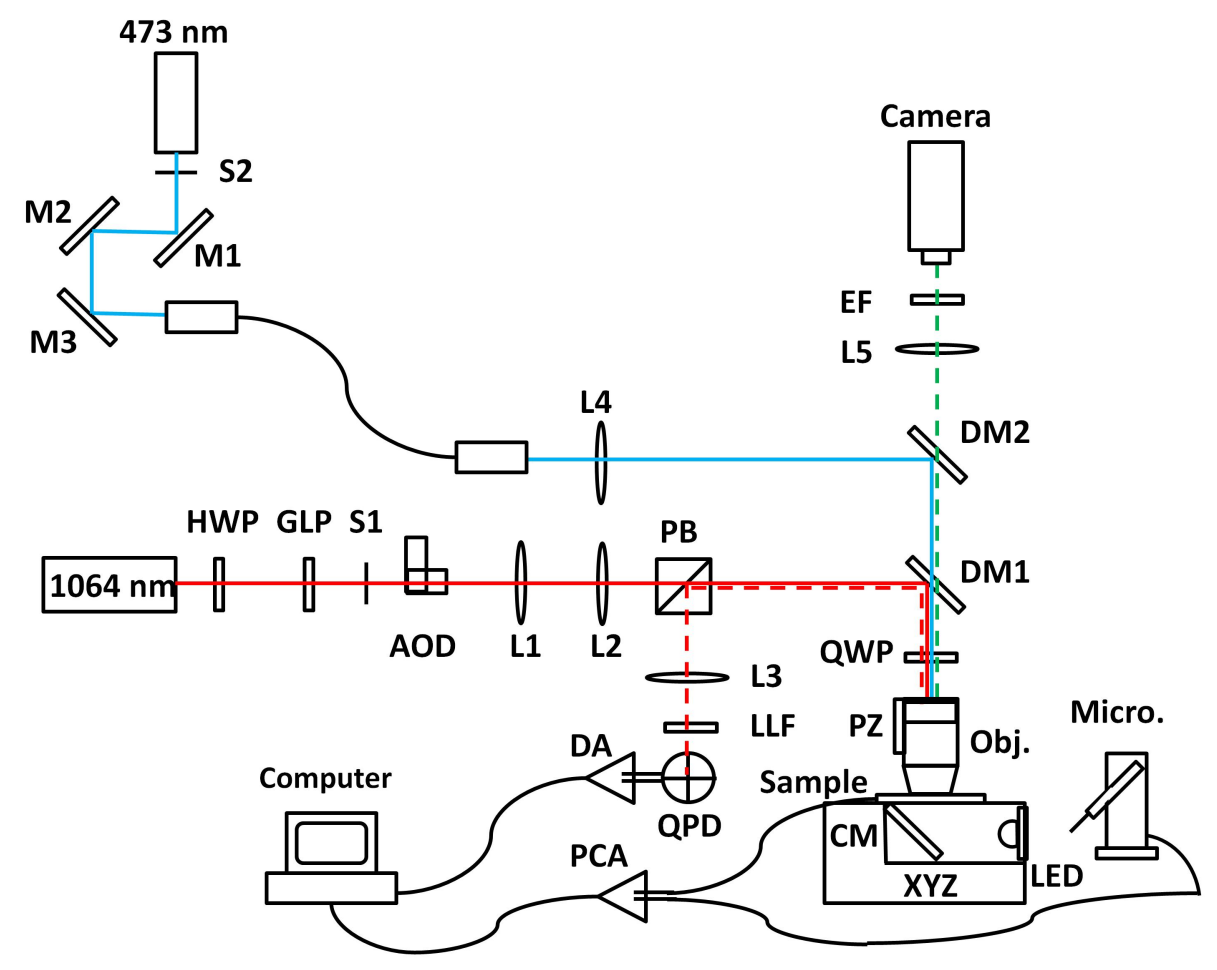

Figure 2.14: Schematic of the experimental setup described in the text. Red solid and dashed lines denote the incident and reflected IR laser beam paths, respectively. The blue solid line denotes the fluorescence laser illumination light path while dashed green lines denote the fluorescence emission light path. S1-S2 indicate shutters in the beam line, which are not included in the description in the text.

respect to the optical table) near-infrared fiber laser $(1064 \mathrm{~nm})$ with a max power of $10 \mathrm{~W}$. A combination of $\lambda / 2$ waveplate (HWP) and Glan-laser polarizer (GLP) is used to adjust beam intensity so that the laser can be operated at a constant power of $\sim 4 \mathrm{~W}$ for stability. The trapping beam is steered laterally via an AOD placed in the objective BFP using a voltage-controlled oscillator which receives commands from a custom-written LabView Virtual Instrument (VI) (Trap Commander). After the AOD, the beam is expanded via a telescope lens pair (L1, L2 ; $\mathrm{f}=75 \mathrm{~mm}$, $\mathrm{f}=150 \mathrm{~mm}$ ) to a spot size of $\sim 8 \mathrm{~mm}$. The trapping light then crosses a polarizing beam splitter (PB), which serves to couple out reflected light into the detection pathway, and is reflected into the microscope objective back aperture by a shortpass dichroic mirror (DM1). A $\lambda / 4$ waveplate (QWP) sits above the objective back aperture and acts to circularly polarize the incoming IR light. Importantly the waveplate is tilted at a slight angle $\left(\sim 2^{\circ}\right)$ to reduce unwanted back-reflections. A 1D piezo stage (PZ) is attached to the objective and allows for axial beam steering. The objective (Obj.) forms a trap in the focal plane and the light which traverses 
the sample passes through a hole in the stage and a long-pass cold mirror (CM), after which it is absorbed by the blacked-out stage body underneath. Reflected IR laser light is collected by the objective and, on re-traversing the QWP, becomes linearly polarized at $90^{\circ}$ to its original orientation ( $s$-polarized). The reflected light continues back along the beam path to $\mathrm{PB}$, where it is reflected into the detection pathway. There, a lens (L3; f=125 mm) focuses the back-scattered light through a laser line filter (LLF) and onto a quadrant photodiode (QPD), which sits in an intermediate image plane. The photodiode is operated at $160 \mathrm{~V}$ reverse bias to guarantee fast response at $1064 \mathrm{~nm}$ wavelength [58]. The QPD signal is amplified by a low-noise, custom-built pre-amplifier and differential amplifier (DA). Analogto-digital conversion diode signal is performed by a field-programmable gate array (FPGA) board, and the signal is recorded using a custom-written LabView VI.

Fluorescence illumination light is provided by a $473 \mathrm{~nm}$ diode-pumped steady state laser, which is coupled into an optical fiber via three mirrors (M1-M3). The fiber output goes through a collimator which is mounted onto the microscope body and which is focused by a lens ( $\mathrm{L} 4 ; \mathrm{f}=125 \mathrm{~mm}$ ) onto the objective $\mathrm{BFP}$ via a dichroic mirror (DM2). The fluorescence signal is collected by the objective and passes DM2, after which it is focused by a lens (L5; $\mathrm{f}=150 \mathrm{~mm}$ ) through an emission filter (EF) onto a CCD camera. Brightfield illumination from below for transparent samples is also possible using a custom-built, multicolor fiber light-emitting diode light source (LED) mounted in the XYZ stage (XYZ). The LED source can be tuned to illuminate the sample with red, green or blue light $(625 \mathrm{~nm}, 525 \mathrm{~nm}$, or $460 \mathrm{~nm})$, or a combination thereof. Brightfield illumination is coupled into the beam path via the CM. The XYZ stage can be translated in all three dimensions by hand using micrometer screws, and its surface can be heated to $40^{\circ} \mathrm{C}$ with a resistive heating element and custom-built temperature controller. A micromanipulator (Micro.) is mounted on the optical table next to the stage, giving the user the option to perform electrophysiological experiments. The manipulator is connected to a patch clamp amplifier (PCA) which is in turn connected via its own AD/DA board to the computer for readout with Patchmaster software. Table 2.1 at the end of this chapter tabulates relevant equipment details, part numbers, and manufacturers.

\section{Performance}

The setup described above is fully functional; it is able to perform brightfield and fluorescence imaging, make electrophysiological measurements, and optically trap microparticles. Figure 2.16 shows side by side images of a monolayer of MDCK cells in brightfield and fluorescence imaging modes. The cells are grown on a flexible sheet, bent over, and the edge is visualized, as described in the Experimental methods chapter. The brightfield image is better than expected, given its simple geometry, 


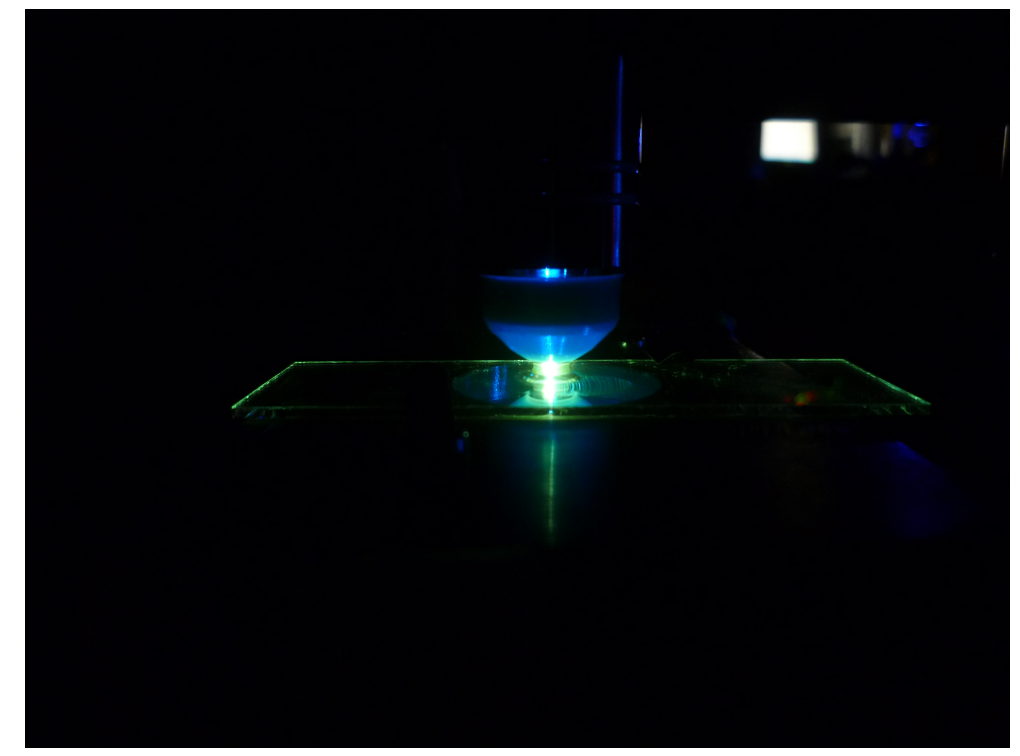

Figure 2.15: Fluorescence imaging in action; a solution of Fluorescein illuminated with blue laser light.
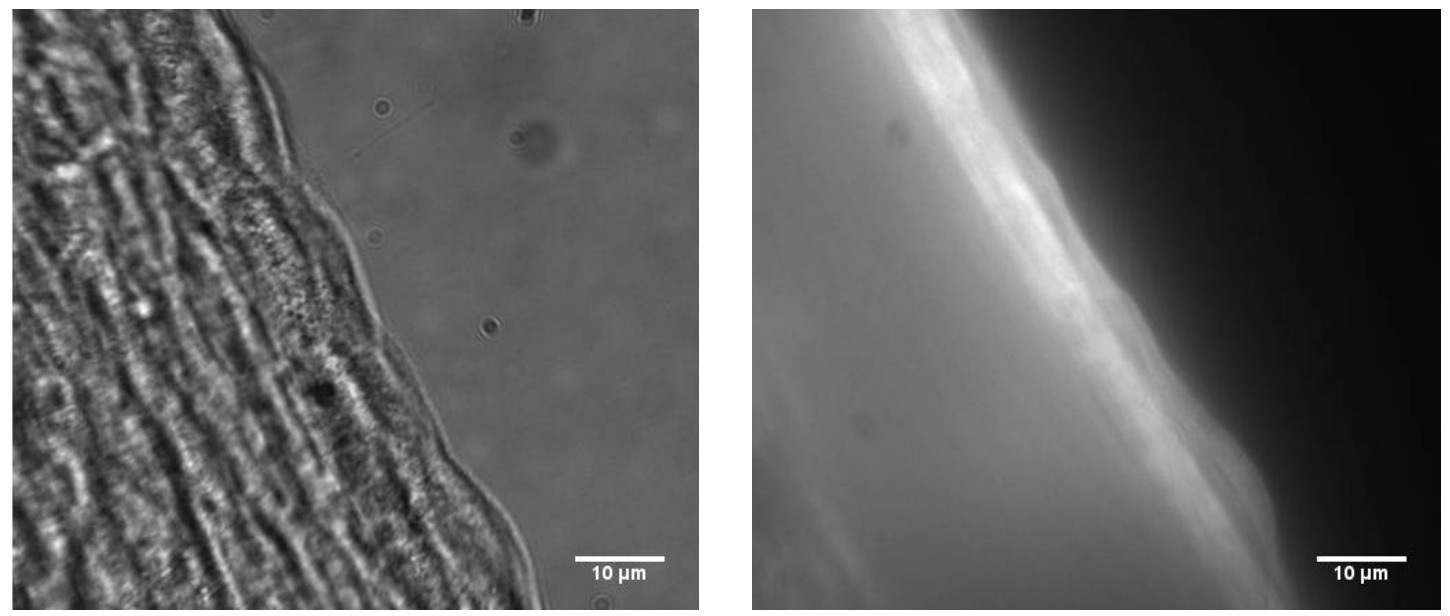

Figure 2.16: Brightfield (left) and fluorescent (right) pictures of an MDCK cell monolayer. The cells are viewed from the side, as described in the Experimental methods chapter, with the microscope focused on the edge of the cell sheet. A cilium is visible in the bright field image, coming out of the second cell from the top, pointing northeast. The cells are stably transfected with a GFP-tagged Somatostatin receptor 3 (SSTR3) plasmid. In these micrographs the NA 1.2 water-immersion objective was used. 


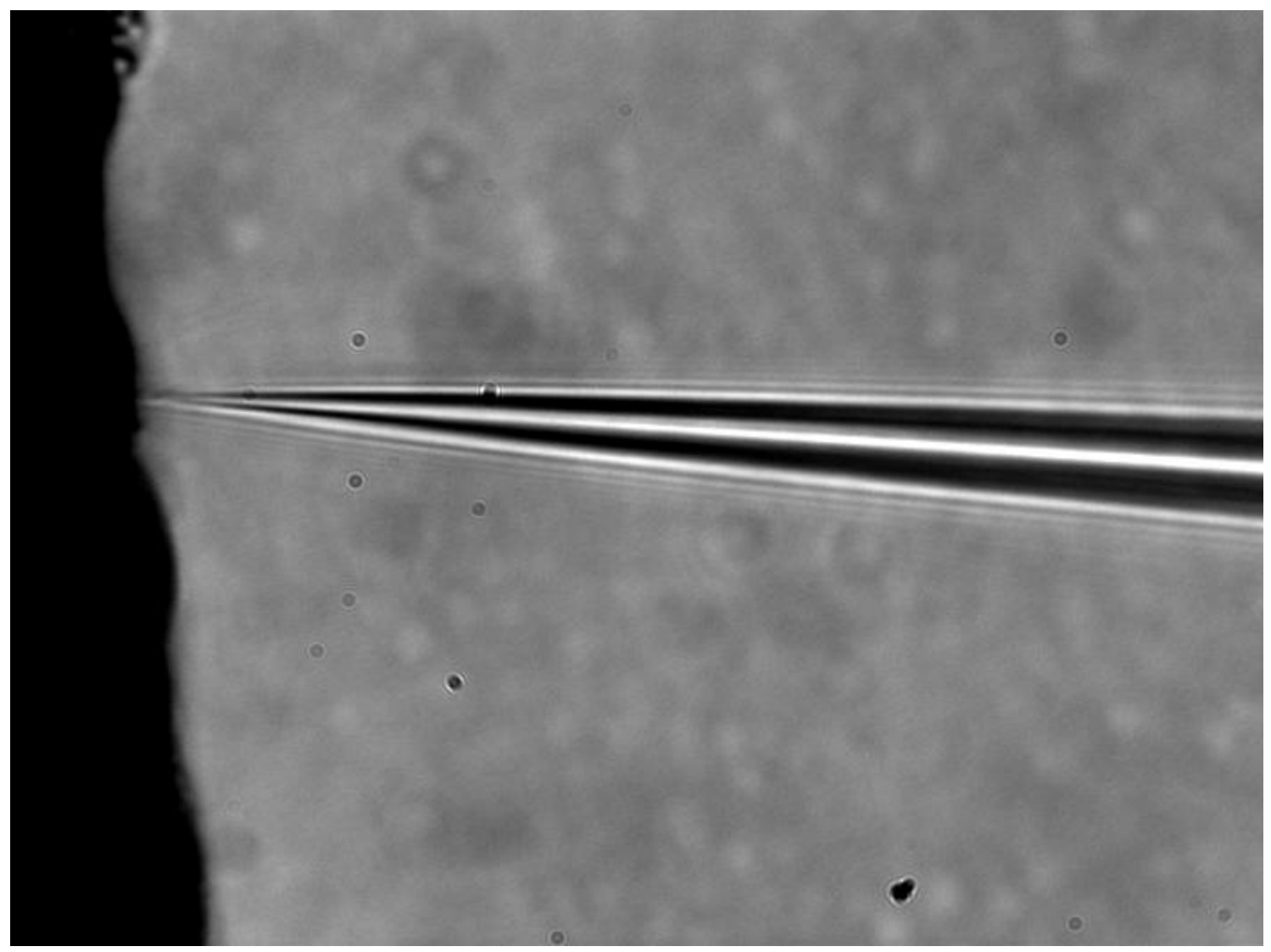

Figure 2.17: Micrograph of sharp electrode recording experiment from a ciliated MDCK cell. The cilium is faintly visible above the pipette, pointing toward the northeast.

and one can even see cilia, which is usually impossible in brightfield microscopy without contrast-enhancing techniques. The sharper contrast is probably due to somewhat oblique illumination of the sample due to imperfect collimation of the light coming out of the LED fiber. The fluorescence image is also good for wide-field epifluorescence, with a reasonably uniform illumination of the entire field-of-view and a strong signal. The image quality is better with the $63 \mathrm{x}$, NA 1.2 water-immersion objective, but is still acceptable with the $100 \mathrm{x}$, NA 1.0 water-dipping objective ${ }^{1}$. Cilia can still be visualized with the latter objective, as can be seen in Figure 2.17.

The micromanipulator can position sharp electrodes and patch clamp pipettes accurately in the sample on the micrometer scale. The manipulator can be moved in $\mathrm{x}, \mathrm{y}$, and $\mathrm{z}$ coordinates, as well as axially along its attachment arm. Despite our

\footnotetext{
${ }^{1}$ The $60 \mathrm{x}$, NA 1.0 water-dipping objective included in the parts list at the end of this chapter is a replacement for the 100x water-dipping objective, but wasn't used in the results presented in this work.
} 


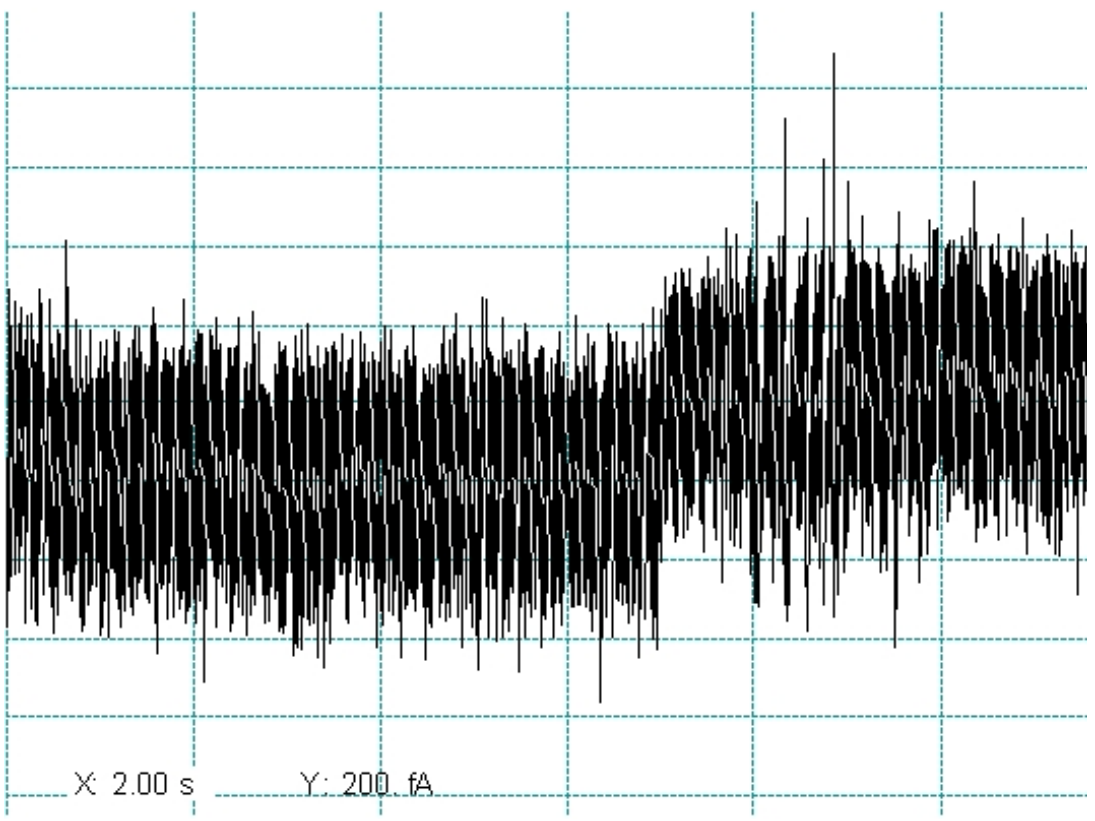

Figure 2.18: Electrophysiological measurement of Gramicidin ion channels reconstituted in a lipid bilayer on a supported substrate. The bilayer composition was DOPC/DPhPC/GramicidinD 88:10:2 with $1 \mathrm{mM} \mathrm{KCl}$ at a holding voltage of $50 \mathrm{mV}$ and low-pass filtered at $100 \mathrm{~Hz}$. Data taken by Theresa Kaufeld.

lack of a low NA objective, usually used in electrophysiology setups to locate the pipette in the microscope field-of-view, finding the pipette in the sample is easily accomplished. First the user positions the micropipette by eye roughly under the objective, then moves the pipette back and forth with the coarse controls. The pipette shadow can be seen moving across the field-of-view, which can then be positioned under the objective and focused on. Figure 2.17 shows a sharp-electrode recording experiment performed on a ciliated MDCK cell. Though the electrophysiological recordings from MDCK cells that we've so far taken have yielded mixed results, electrophysiological measurements can be performed (see Chapter 6). The patch clamp amplifier and acquisition software have also been used to take electrophysiological data from supported lipid bilayers. Figure 2.18 shows a recording from a 

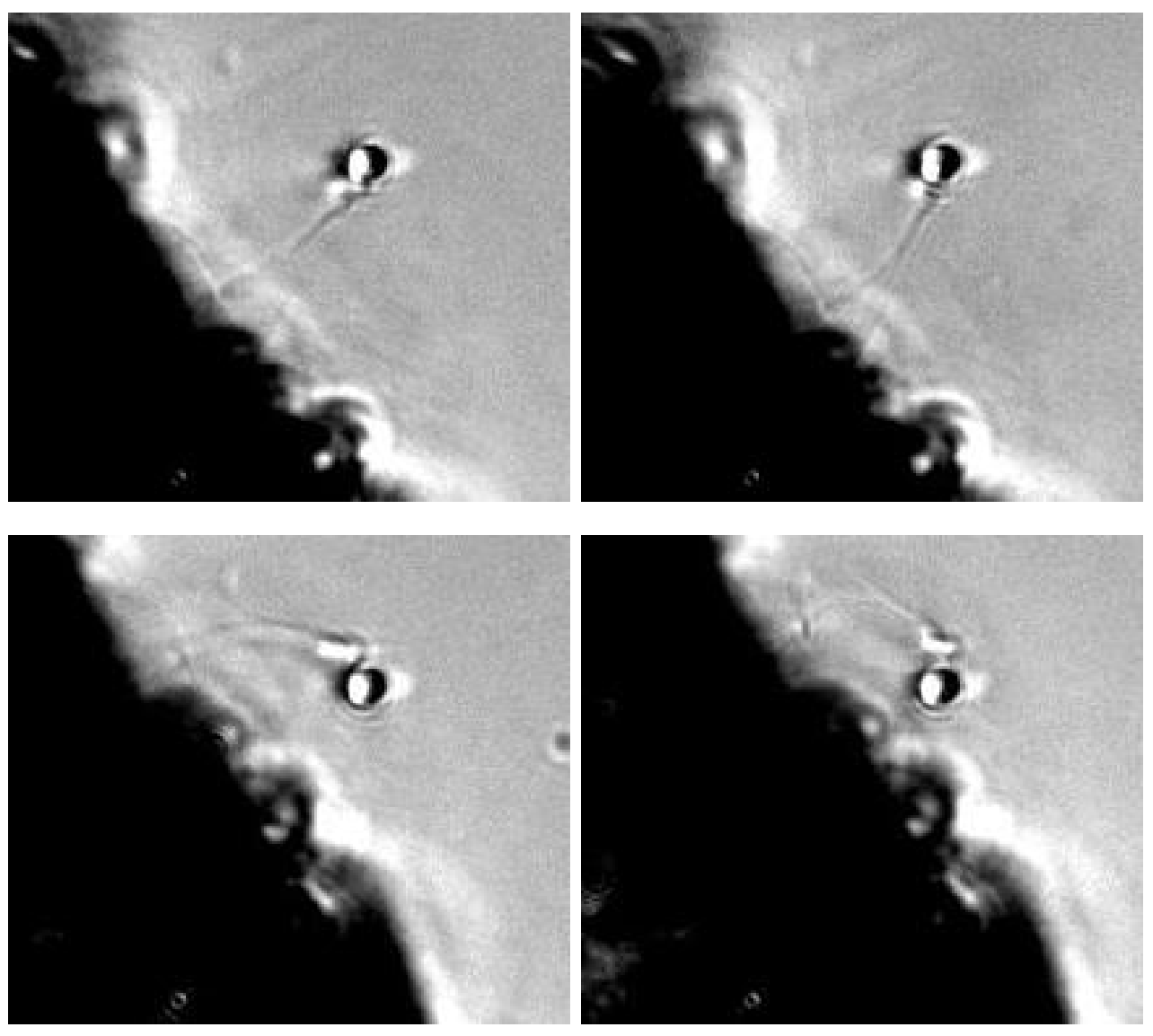

Figure 2.19: Series of stills of an MDCK cilium bent by an optical trap. A $1.5 \mu \mathrm{m}$ silica bead is attached to the cilium and it is bent back and forth by moving the trap. The NA 1.0 water-dipping objective was used in this experiment and the cells were imaged in an edge-viewing geometry.

lipid bilayer with reconstituted Gramicidin ion channels taken by Theresa Kaufeld. The trace shows a spontaneous single-channel opening event $(\sim 13 \mathrm{pS})$. Due to the very low noise (below $1 \mathrm{pA}$ ), even such low-conductance events can be resolved.

The optical trap, which plays a key role in the capabilities of our instrument, is able to trap and manipulate microscopic objects, as shown in Figure 2.19. The pictures come from a series of stills in which a $1.5 \mu \mathrm{m}$ bead was attached to a cilium and bent back and forth with the AOD. Forces up to the order of $10 \mathrm{pN}$ can be generated with appropriate powers and beads, although much lower forces are needed to bend cilia $(<1 \mathrm{pN})$. Our BSD system is able to detect the scattered signal from micron-sized beads, as evinced in Figure 2.20, a lateral AOD scan of a $1.5 \mu \mathrm{m}$ bead affixed to a glass slide ${ }^{2}$. The response curve looks as expected (e.g. like an FSD

\footnotetext{
${ }^{2}$ All the following measurements were performed with the NA 1.0 water-dipping objective,
} 


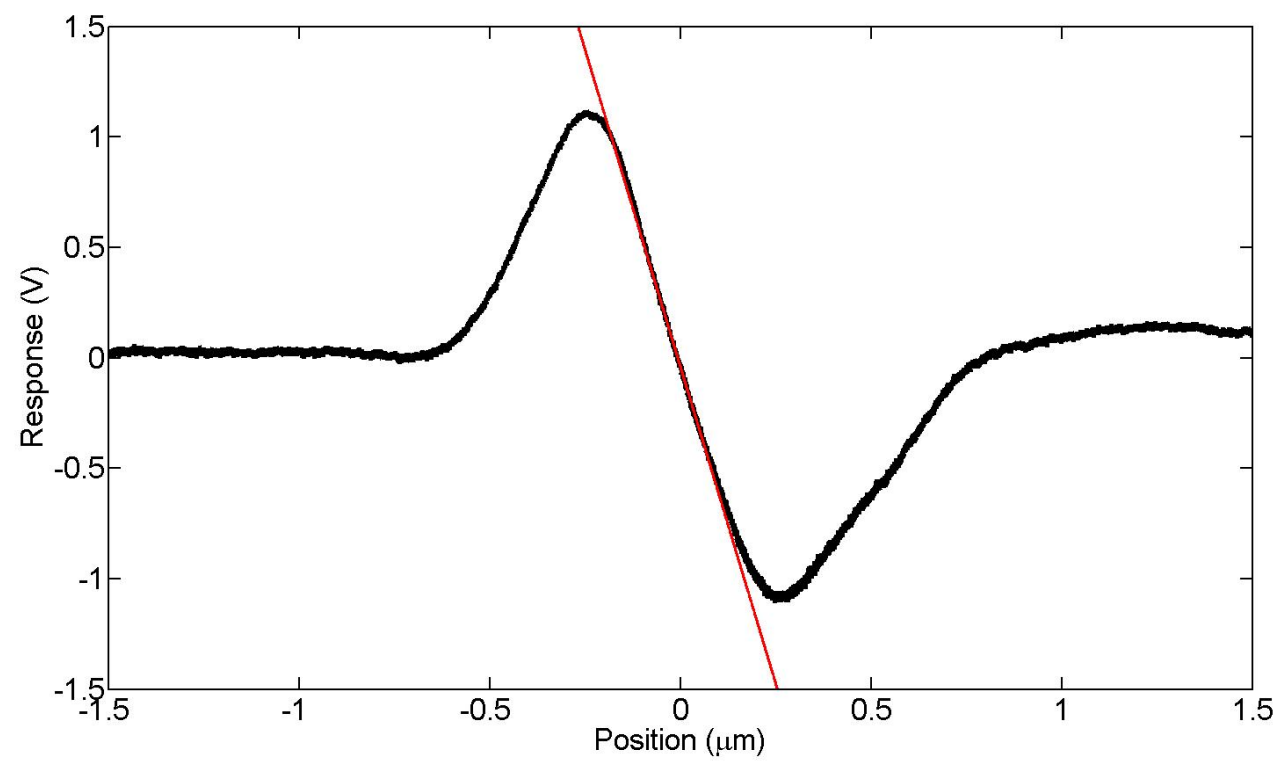

Figure 2.20: QPD response from lateral scan of a $1.5 \mu \mathrm{m}$ silica bead fixed to a glass slide. The red line is a linear fit to the middle region of the response curve. The response is linear to within $\pm 5 \%$ over $\sim 200 \mathrm{~nm}$.

response curve) and has a similar range of linearity $(\sim 200 \mathrm{~nm})$. The axial response was also measured and found to be as expected, as shown earlier in Figure 2.11. Unfortunately, due to the weak scattered signal, beads smaller than $1.5 \mu \mathrm{m}$ are difficult to detect in our setup, at least with the NA 1.0 water-dipping objective. Since the back-scattered signal is so small, it is critical to minimize unwanted back reflections from other optical components. Lateral and axial power spectra for different trapped beads can be seen in Figures $2.21 \& 2.22$. The reader may note that the corner frequency, and consequently the trap stiffness, is higher in Figure 2.21. This is due to the fact that in the first measurement more power was used than in the second. The noise floor (i.e. the flattening out at high frequencies) is also higher in the second spectrum for the same reasons. Figure 2.21 demonstrates that higher strength trapping is possible in our setup, although the trapping strengths we typically used were lower. The power spectrum depicted in Figure 2.22, while noisier, shows that axial detection is possible, even given the weak back-scattered signal ${ }^{3}$.

To better characterize our axial signal we performed axial scans of beads fixed to

except for the one depicted in Figure 2.21.

${ }^{3}$ The lateral power spectra of the bead in Figure 2.22 were similar in shape and trapping strength (data not shown). 


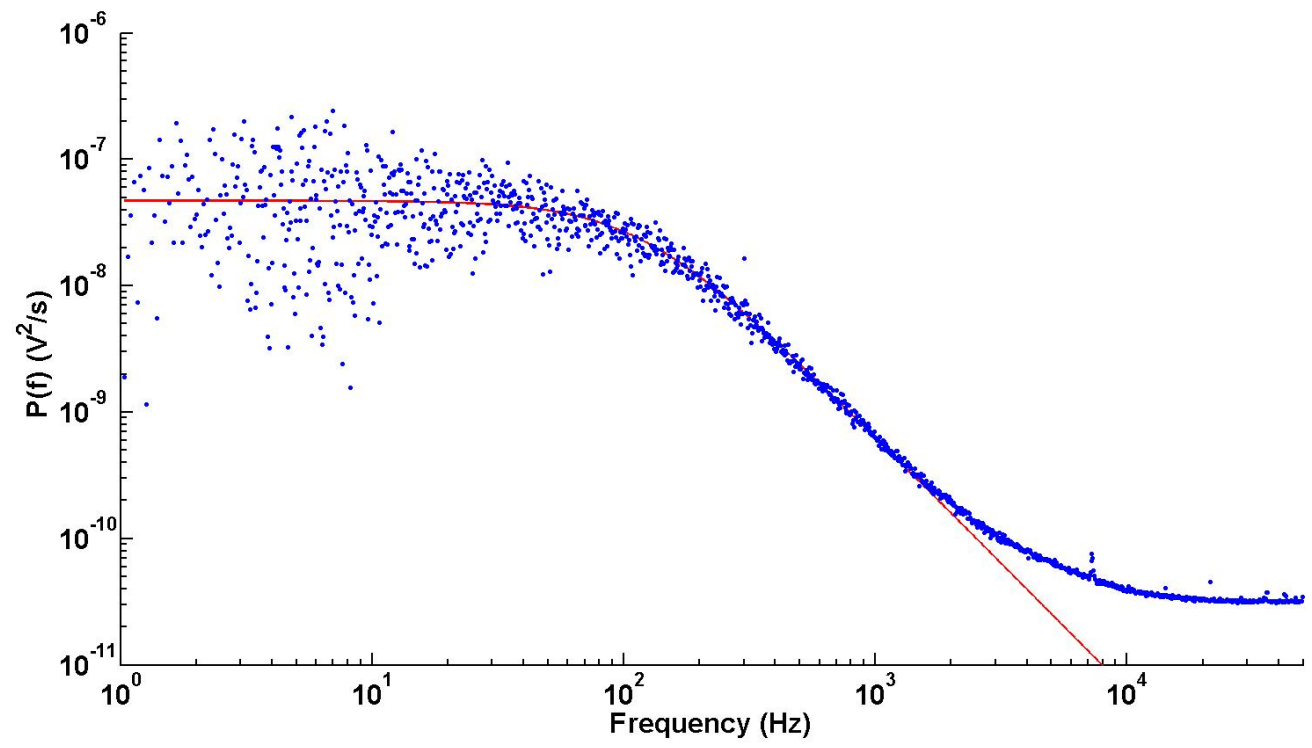

Figure 2.21: One-sided power spectrum of a trapped $2 \mu \mathrm{m}$ polystyrene bead's lateral fluctuations in BSD using an NA 1.2 water-immersion objective. The blue dots represent block averaged, log-binned data while the solid red line is the fitted Lorentzian. Fit done using the MatLab package TweezerCalib described in Ref. [57].

a glass slide, as well as "pushing experiments" in which trapped beads were pushed against the bottom of the sample chamber with our piezo stage until they popped out of the trap. The purpose of the axial scan was to characterize the QPD response curve of our axial signal and to test the piezo stage. The result is the red curve in Figure 2.23. As one approaches the stuck bead from above (left to right) one sees a very slight increase of signal, a small oscillation, and then the large, familiar response peak. The increase in signal is due to a greater proportion of light being reflected back and collected closer to the interface, while the small oscillation is probably due to interference between the bead surfaces. In contrast, the black curve in Figure 2.23 has significant differences. It is the result of a "pushing experiment" which was done in anticipation of similar experiments to measure tension in supported lipid bilayers. While the overall trend is the same as in the axial scan curve, the pushing curve is modulated by a periodic signal which increases in amplitude with decreasing distance from the sample chamber's bottom. These oscillations are due to wave interference between the bottom interface and the bead surfaces, similar to an etalon where the distance between the two plates is continuously varied [30,34]. This oscillating signal is a double-edged sword; on the one hand it can be used as a very sensitive measure of how close to the bottom interface the bead is, while on the other hand 


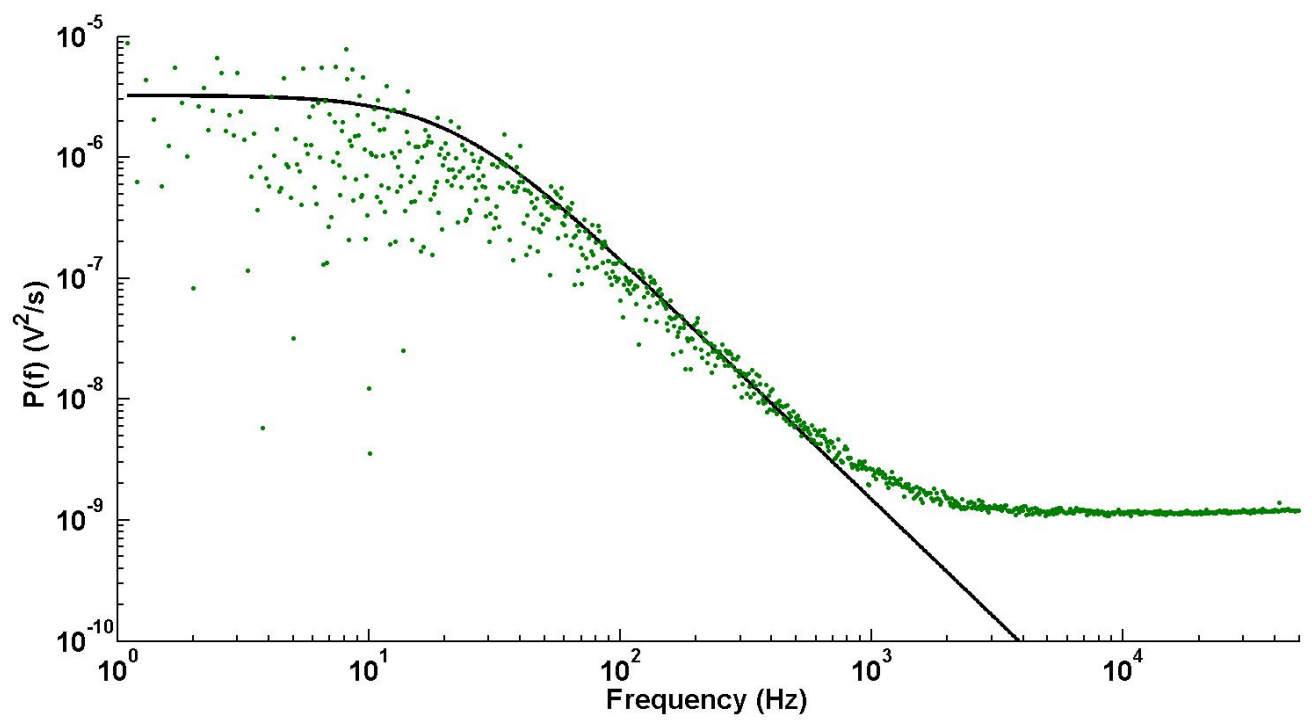

Figure 2.22: One-sided power spectrum of a trapped $1 \mu \mathrm{m}$ silica bead's axial fluctuations in BSD using an NA 1.0 water-dipping objective. The green dots represent block averaged, log-binned data while the solid black line is the fitted Lorentzian. Fit done using the MatLab package TweezerCalib described in Ref. [57].

it introduces a large noise signal into relative vertical position/force measurements. While the oscillations can't be removed altogether, they can be mitigated with the introduction of a central beam obstruction (which makes the beam profile "donutlike") and by spatial filtering with a pinhole in the detection pathway [34]. While initial attempts to reduce signal oscillations with a central obstruction have been promising, an optical thickness gradient across the obstruction is needed to avoid sharp-edge diffraction effects, which reduce beam quality.

\section{Discussion}

This setup was initially built to perform an ambitious experiment, wherein a bead would be attached to the primary cilium of an MDCK cell, the cell would be patch clamped, the primary cilium would be mechanically stimulated with the optical trap, and the mechanosensitive response would be read out via the patch electrode. In order to visualize cilia well, we planned to transfect MDCK cells with fluorescent proteins which localized to the cilium. The sister experiment for which the setup was also designed consisted of expressing an ion channel endemic to the cilium (PC2) in a substrate-supported lipid membrane and gating the channel through mechanical 


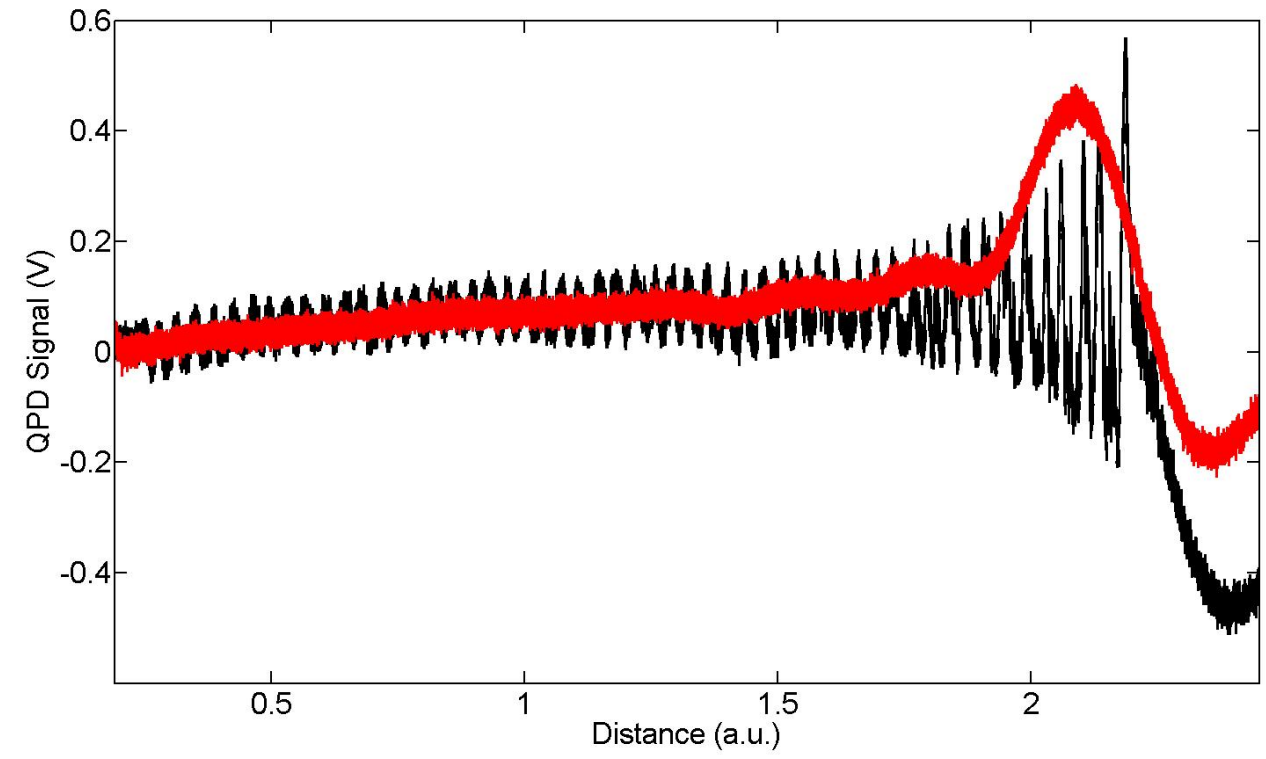

Figure 2.23: Comparison of QPD axial responses. The red curve shows the QPD signal from an axial scan of a $1.5 \mu \mathrm{m}$ silica bead fixed to a glass slide while the black curve shows the QPD signal from a $1.5 \mu \mathrm{m}$ silica bead trapped and pushed against the microscope slide until it escapes the trap. The signal oscillations in the pushing curve are due to standing wave interference between the bead and the glass surface. Both scans performed with the NA 1.0 water-dipping objective.

stimuli applied by the optical trap. Though progress was made in both projects, neither has come to full fruition. Indeed the first experiment, which the author intended to perform, seems to be prohibitively difficult. While we were able to overcome the technical hurdles of attaching beads to cilia, visualizing them, and making them fluorescent, patch clamping them has proven infeasible. While there is extensive literature on patch clamping isolated MDCK cells, when they form the confluent monolayers needed to express cilia they become very difficult to patch clamp. Given the inherent difficulties of adding to an already difficult experiment more "moving parts" (i.e. bead attachment, locating a fluorescing cilia, etc.), we decided to abandon the original approach and instead concentrated our efforts on sharp electrode recording and calcium imaging to monitor the mechanosensitive response. In addition, it became clear that the cilium mechanics and dynamics were an interesting story in their own right, and it is they that compose the main results of this work. To follow the cilium dynamics we built DIC optics into a second optical trap and wrote an algorithm to track DIC line images, the specifics of which comprise the next two chapters. Somewhat ironically, while the building of this setup took 
up a large part of the time this work chronicles, it wasn't used in acquiring the most important results reported here. Its electrophysiological capacities have been used successfully by Theresa Kaufeld to monitor the electrical properties of substratesupported bilayers on chips with individually-addressable multi-electrode arrays, a first step toward the sister experiment mentioned above. We plan to explore the mechanosensitive response of individual ciliated cells using either calcium imaging or sharp electrode recording with this setup in the future. 
Table 2.1: List of Setup Components

\begin{tabular}{|c|c|c|}
\hline Component & Part Name/Notes & Manufacturer \\
\hline NIR laser & YLD-10-1064-LD & IPG Photonics Corp., Oxford, MA, USA \\
\hline & $10 \mathrm{~W} \max$ power, $1064 \mathrm{~nm}, \mathrm{cw}$ & \\
\hline HWP & WPH05M-1064 & Thorlabs Inc., Newton, NJ, USA \\
\hline GLP & GL10-C26 & Thorlabs Inc., Newton, NJ, USA \\
\hline $\mathrm{AOD}$ & DTSXY-400-1064 & AA Optoelectronic, Orsay Cedex, France \\
\hline L1, L2, L3, L4, L5 & achromatic doublets & Thorlabs Inc., Newton, NJ, USA \\
\hline QWP & WPQ05M-1064 & Thorlabs Inc., Newton, NJ, USA \\
\hline PBS & PBS203 & Thorlabs Inc., Newton, NJ, USA \\
\hline PZT & P-721.CDQ PIFOC & Physik Instrumente GmbH, Karlsruhe, Germany \\
\hline \multirow[t]{3}{*}{ Objectives } & $\begin{array}{l}\text { LUMPlanFL } \mathrm{W}, 100 \mathrm{x} \\
\text { NA } 1.0 \text {, water-dipping }\end{array}$ & Olympus Corp., Tokyo, Japan \\
\hline & $\begin{array}{l}\text { LUMPlanFL N, } 60 \mathrm{x} \text {, } \\
\text { NA 1.0, water-dipping }\end{array}$ & Olympus Corp.,Tokyo, Japan \\
\hline & $\begin{array}{l}\text { C-Apochromat, } 63 \mathrm{x} / 1.2 \mathrm{~W} \\
\text { NA } 1.2, \text { water-immersion }\end{array}$ & Carl Zeiss AG, Jena, Germany \\
\hline XYZ Stage & $562-\mathrm{XYZ}$ & Newport Co., Irvine, CA, USA \\
\hline M1, M2, M3 & P01 & Thorlabs Inc., Newton, NJ, USA \\
\hline $\mathrm{CM}$ & $62-636$ & Edmund Optics Ltd., York, UK \\
\hline DM1 & $495 \mathrm{dcxt}$ & Chroma Technologies, Bellows Falls, VT, USA \\
\hline DM2 & z1064rdc-sp & Chroma Technologies, Bellows Falls, VT, USA \\
\hline $\mathrm{EF}$ & HQ525/50m-2p & Chroma Technologies, Bellows Falls, VT, USA \\
\hline LLF & FL051064-10 & Thorlabs Inc., Newton, NJ, USA \\
\hline PCA. & ELC03XS & npi Electronic GmbH, Tamm, Germany \\
\hline Micro. & PS-7000C & Scientifica Ltd., Wokingham, UK \\
\hline QPD & SPOT-9-YAG & OSI Optoelectronics, Hawthorne, CA, USA \\
\hline Fiber \& & $60 \mathrm{FC}-4-\mathrm{A} 6.2-01$ & Schäfter+Kirchhoff GmbH, Hamburg, Germany \\
\hline collimation optics & 60FC-T-4-M60-04, SMC-460 & \\
\hline Mirror holders & SN100-F3K & Newport Co., Irvine, CA, USA \\
\hline CCD Camera & CSNAP-EZ-M-FW-12-PC-UNV & Roper Industries Inc., Sarasota, FL, USA \\
\hline Blue DPSS Laser & VA-I-N-473, 50mW, $473 \mathrm{~nm}$ & Beijing Viasho Technology Co.,Ltd., Beijing, China \\
\hline LED & $\begin{array}{l}470 \mathrm{~nm} \mathrm{w} / 70 \mathrm{~mW} \\
525 \mathrm{~nm} \mathrm{w} / 50 \mathrm{~mW}, \\
625 \mathrm{~nm} \mathrm{w} / 70 \mathrm{~mW}\end{array}$ & Doric Lenses, Quebec City, Canada \\
\hline FPGA & PCI-7833R & National Instruments, Austin, TX, USA \\
\hline $\mathrm{AD} / \mathrm{DA}$ board & InstruTECH LIH $8+8$ & $\begin{array}{l}\text { HEKA Elektronik Dr. Schulze GmbH, } \\
\text { Lambrecht/Pfalz, Germany }\end{array}$ \\
\hline LabView & software & National Instruments, Austin, TX, USA \\
\hline Patchmaster & software & $\begin{array}{l}\text { HEKA Elektronik Dr. Schulze GmbH, } \\
\text { Lambrecht/Pfalz, Germany }\end{array}$ \\
\hline
\end{tabular}




\section{Chapter 3}

\section{Incorporation of DIC microscopy into a dual-optical trap}

In the micromanipulation experiments described in this thesis image quality is essential, as a large part of the data we analyze is taken from micrographs of the primary cilium. Though fluorescence is the king of modern biological light microscopy techniques, thanks to the proliferation of genetically encodable fluorescent proteins, we used an alternate imaging modality for much of our data collection, namely differential interference contrast (DIC) microscopy. This choice was primarily driven by intially unsuccessful attempts at fluorescent labeling of primary cilia, but in the end had several advantages over fluorescence. In this chapter we describe how we incorporated DIC into an existing dual-optical trap setup and the attendant subtleties. We start with a brief review of DIC image formation, followed by a technical description of the setup, and then show how one can correct for depolarization effects which degrade image quality with the judicious insertion of several optical elements. We conclude with a discussion of the benefits and drawbacks of our modified system. The technique described in this chapter for correcting depolarization effects was the subject of a paper accepted for publication in the journal Review of Scientific Instruments.

\section{DIC Microscopy}

DIC microscopy is a common mode of biological light microscopy used to achieve maximal resolution and contrast with weakly absorbing specimen such as cells. First devised by Francis Smith in 1955, it offers an easily implemented, label-free way to resolve sub-diffraction limit details in biological specimens at video rates without limitations such as sample bleaching [59]. Any microscope that can accept polarizing filters and the specialized prisms necessary for DIC can be converted to use this technique. The main principle behind DIC, and indeed all types of phase 


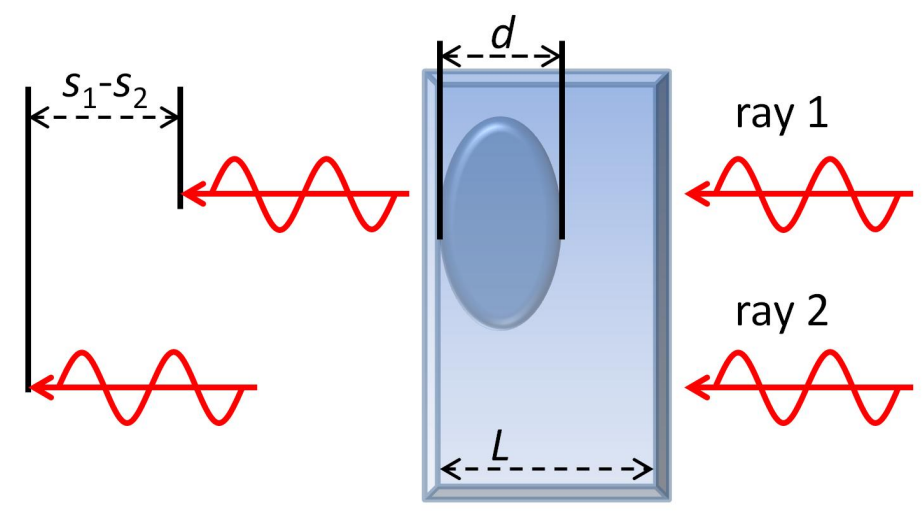

Figure 3.1: Schematic of phase lag due to a specimen of a different refractive index than the surrounding medium. Light from ray 1 travels through the specimen with a refractive index, $n_{s}$, different from the refractive index of the medium, $n_{m}$, thus acquiring a relative phase shift, $\delta$, proportional to the optical path difference, $s_{1}-s_{2}$, of the two rays, while ray 2 only passes through the medium.

contrast microscopy techniques, is to convert phase differences arising from diffraction/refraction of the illuminating light by the sample into intensity differences in the final image. In transparent samples such as cells in an aqueous environment, phase gradients generally arise due to the difference in refractive index between the sample and the surrounding medium, as well as from thickness variations in the sample itself. Figure 3.1 shows a diagram in which two different light rays from a monochromatic source travel through a sample and experience different refractive indices, thus acquiring a phase shift relative to one another. The optical path lengths of the two rays, given by the speed of light in a vacuum multiplied by the time it takes a light ray to traverse a given distance [42], are

$$
\begin{aligned}
& s_{1}=n_{s} d+n_{m}(L-d) \\
& s_{2}=n_{m} L
\end{aligned}
$$

where $n_{s}$ is the refractive index of the sample, $n_{m}$ is the refractive index of the surrounding medium, $d$ is the thickness of the sample, and $L$ is the total thickness of the chamber containing medium and sample. It is then clear that the phase difference between the two rays due to the sample is given by

$$
\delta=2 \pi\left(s_{1}-s_{2}\right) / \lambda
$$

where $\lambda$ is the wavelength of the incident rays. Thus to make use of phase gradients 
in the sample, one needs illumination light with a spatially and temporally fixed phase relation to capture the information encoded in the thickness/refractive index differences, as well as a method to transform this phase information into intensity differences visible to the eye or a photosensor. There are a multiplicity of ways to do this, e.g. phase contrast microscopy, Hoffman-modulation contrast microscopy, etc., and DIC solves the problem by using the interference of two different, slightly offset images with each other to generate contrast. A typical DIC optical train is shown in Figure 3.2a. The important steps for image formation are as follows [60]:

1. Light from the illumination source is polarized by a polarizer. This ensures a fixed polarization relationship which is exploited to shear the illumination beam into two equal intensity components.

2. The polarized light passes through a special birefringent prism (Prism 1), generally a Nomarski-modified Wollaston prism in modern microscopes, which is placed near the back focal plane (BFP) of the condenser. This prism is usually made of wedges of quartz cemented together in such a way so that the optical axes of the quartz wedges make an oblique angle with one another [59]. The prism is oriented at $45^{\circ}$ to the polarization axes of the illumination light, causing an angular splitting, or shear, of the incoming light into two orthogonally polarized components, generally termed the ordinary and extraordinary components. This splitting leads to a spatial separation of the ordinary and extraordinary wavefronts, typically of the order of several hundred nanometers (greatly exaggerated in the schematic). Since each light ray that is split came from a localized area of the illumination source, they are coherent, thus giving them the identical phase and amplitude relationships necessary to exploit phase gradients in the sample.

3. The light is collected by the condenser and the angular separation of the extraordinary and ordinary rays is transformed into spatial separation. This spatial separation is less than the diameter of the Airy disc at that magnification.

4. The ordinary and extraordinary wavefronts pass through the sample, offset by an amount given by their relative shear, and each wavefront experiences optical path lengths dictated by the local refractive indices/specimen thicknesses that they encounter and the corresponding phase shifts.

5. Light passing through the sample is collected by the objective and passes through an identical Nomarski-modified Wollaston prism (Prism 2) situated near the BFP of the objective. This prism merges the extraordinary and ordinary rays into two parallel wavefronts, which are out of phase by an amount dictated by their optical path difference. This prism can often be translated to 
a

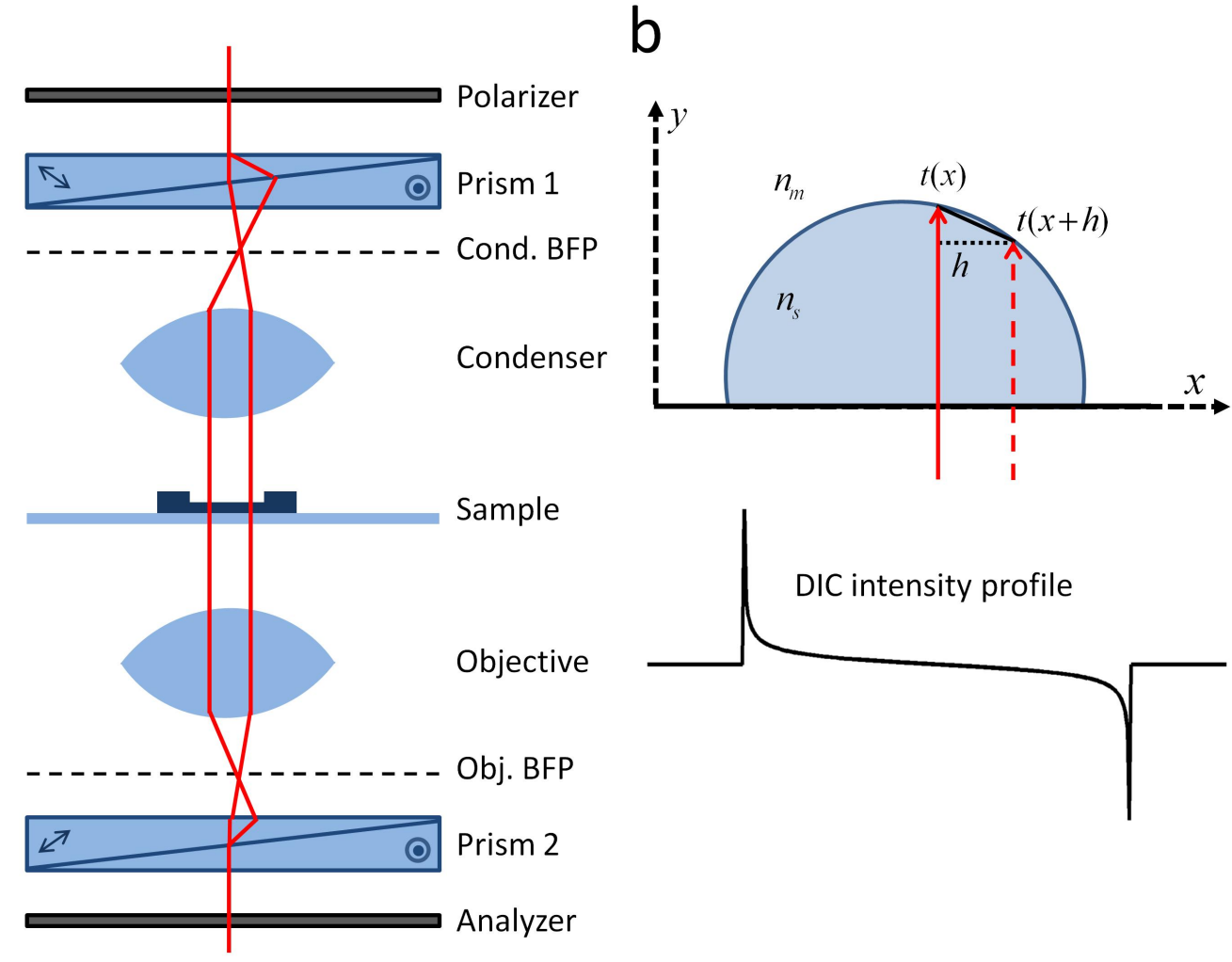

Figure 3.2: a) Schematic of the DIC optical train described in the text. Briefly, illumination light comes from above, is polarized by the polarizer, split by Prism 1, passes through the sample, is recombined by Prism 2, and interferes at the analyzer to produce an interferometric image of the phase gradients in the sample. b) Schematic of a specimen traversed by extraordinary and ordinary rays and the resultant DIC profile. The solid ray represents the extraordinary beam while the dashed ray represents the ordinary beam. The two rays traverse the sample separated by the shear distance $h$, encountering thicknesses of $t(x)$ and $t(x+h)$. When they're recombined at the analyzer, the phase difference is proportional to a discrete estimate of the gradient (solid black line) as described in the text, resulting in a DIC profile like the one shown. 


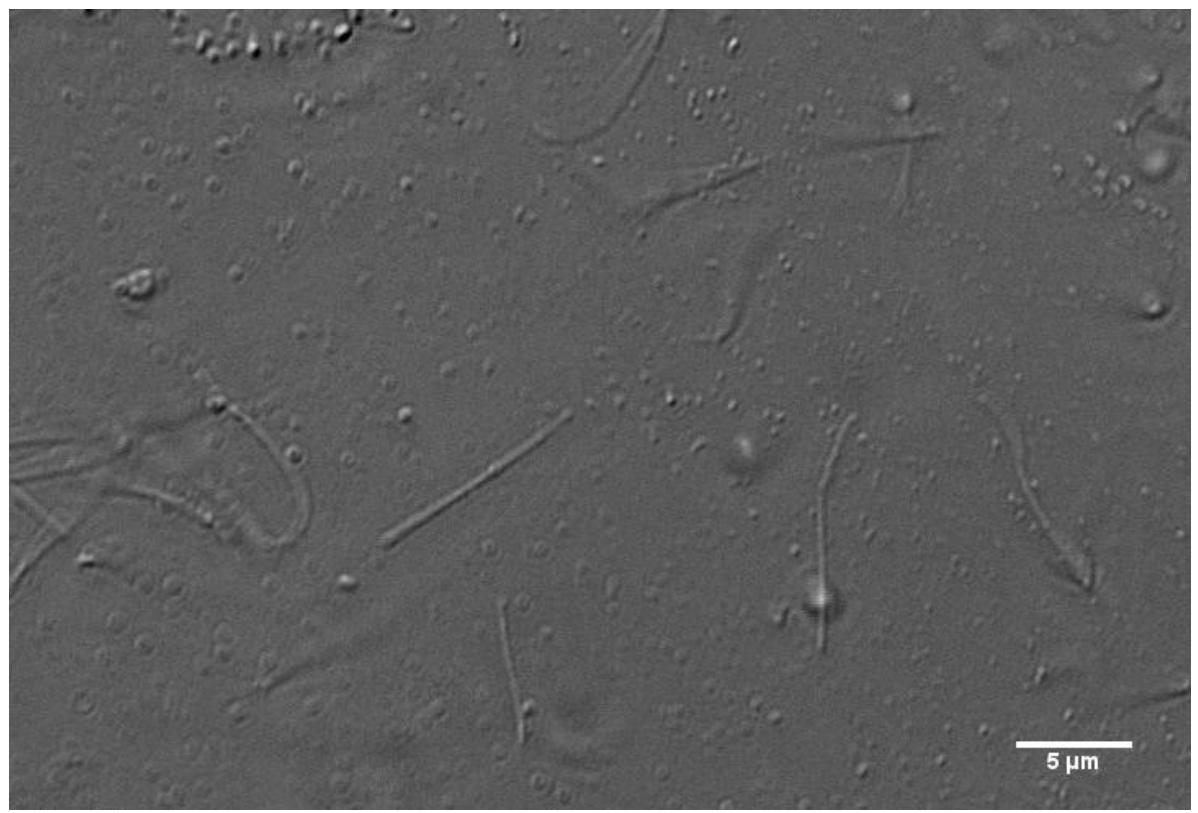

Figure 3.3: DIC image of Chlamydomonas reinhardtii axonemes adhered to a glass coverslip. In the image it appears as if their is a shadow cast from the northwest to southeast, due to the bias of the objective DIC prism. Note that this implies that the shear axis of the prism is oriented northwest to southeast.

introduce "bias", i.e. an additional phase advancement/retardation between the two orthogonal wavefronts. This additional delay arises from the different optical path differences achievable by translating a wedge geometry. When the prism is moved off-center, the light will traverse more/less of the upper quartz wedge, which in turn induces a phase shift between the extraordinary and ordinay components, due to the optical anistropy of the material. This phase shift can be tuned to give more contrast in the final DIC image.

6. Finally the light passes through the analyzer, a second polarizer oriented at $90^{\circ}$ to the first polarizer, where the two components are recombined into the same vibrational plane, and interference between the phase-shited wavefronts occurs, thus achieving the desired transformation of phase differences into intensity differences.

The resultant image has a pseudo 3D quality, with one side of the specimen typically light and the opposite side dark as if the specimen were obliquely illuminated, as can be seen in Figure 3.3. It should be noted that, since DIC images are the result of optical path differences which are themselves the product of the specimen thickness and refractive index difference, without independent information one can't associate the phase diferences imaged with geometrical differences in the 
specimen. Furthermore, the intensity profile of the image formed is of the gradient of the optical path difference, rather than of the optical path difference itself $[61,62]$. This can be understood as follows: since the illumination light is (initially) linearly polarized, the crossed analyzer forces all recombined wavefronts that are a multiple of $\pi$ out-of-phase with each other to zero. If they are out of phase by some other angle, the resultant wavefront is elliptically polarized and so a portion of it will pass the analyzer to form an image in the image plane. The amount of light passing the analyzer is given by the relative phase difference between the extraordinary and ordinary rays, which is porportional to the gradient of the optical path difference. Figure $3.2 \mathrm{~b}$ shows a simple example of this: a hemispherical specimen of a uniform refractive index $n_{s}$ in a medium of refractive index $n_{m}$. In this case the optical path difference at a point $x$ is simply $t(x)\left(n_{s}-n_{m}\right)$. From Eqn. 3.2 we see that the phase difference between extraordinary and ordinary rays is

$$
\delta_{d i f f}=\delta_{o}-\delta_{e}=\frac{2 \pi\left(n_{s}-n_{m}\right)}{\lambda}(t(x+h)-t(x))
$$

where $\delta_{e}$ is the extraordinary ray phase shift, $\delta_{o}$ is the ordinary ray phase shift, $h$ is the shear distance, $t(x)$ is the specimen thickness traversed by the extraordinary ray, and $t(x+h)$ is the specimen thickness traversed by the ordinary ray. It's clear from the suggestive notation above that $\delta_{\text {diff }} \propto \Delta_{h}[t](x)=t(x+h)-t(x)$, the forward finite difference of $t$, a discrete approximation of the derivative of $t$ when divided by small values of $h$. Since shear values are often on the order of the wavelength or less, $\delta_{\text {diff }} \propto \Delta_{h}[t](x) / h \approx d t / d x$, so the phase difference, and thus the intensity of the DIC image, follows the gradient of the optical path difference rather than the difference itself.

Given the image formation process of a DIC microscope discussed above, it's clear that maintaining the correct polarization state of the illumination light is critical to image quality, as it determines contrast in the final image. Unwanted depolarization degrades image quality, which is a potential problem when one uses a dual-optical trap where the traps are created from orthogonal polarization states of a single laser. Incorporating DIC into such a setup is complicated by the need to keep the two trapping beams from being split further by the Wollaston prisms, while simultaneously keeping the illumination light linearly polarized after reflection from/transmission through the dichroic mirrors. Splitting of the trapping lasers would create two pairs of traps, or effectively two elongated anisotropic traps, and it creates cross-talk between the two beams, while depolarization of the illumination light leads to image degradation. In the next section we describe the details of our DIC/dual-optical trap microscope, and then how to fix unwanted depolarization effects. 


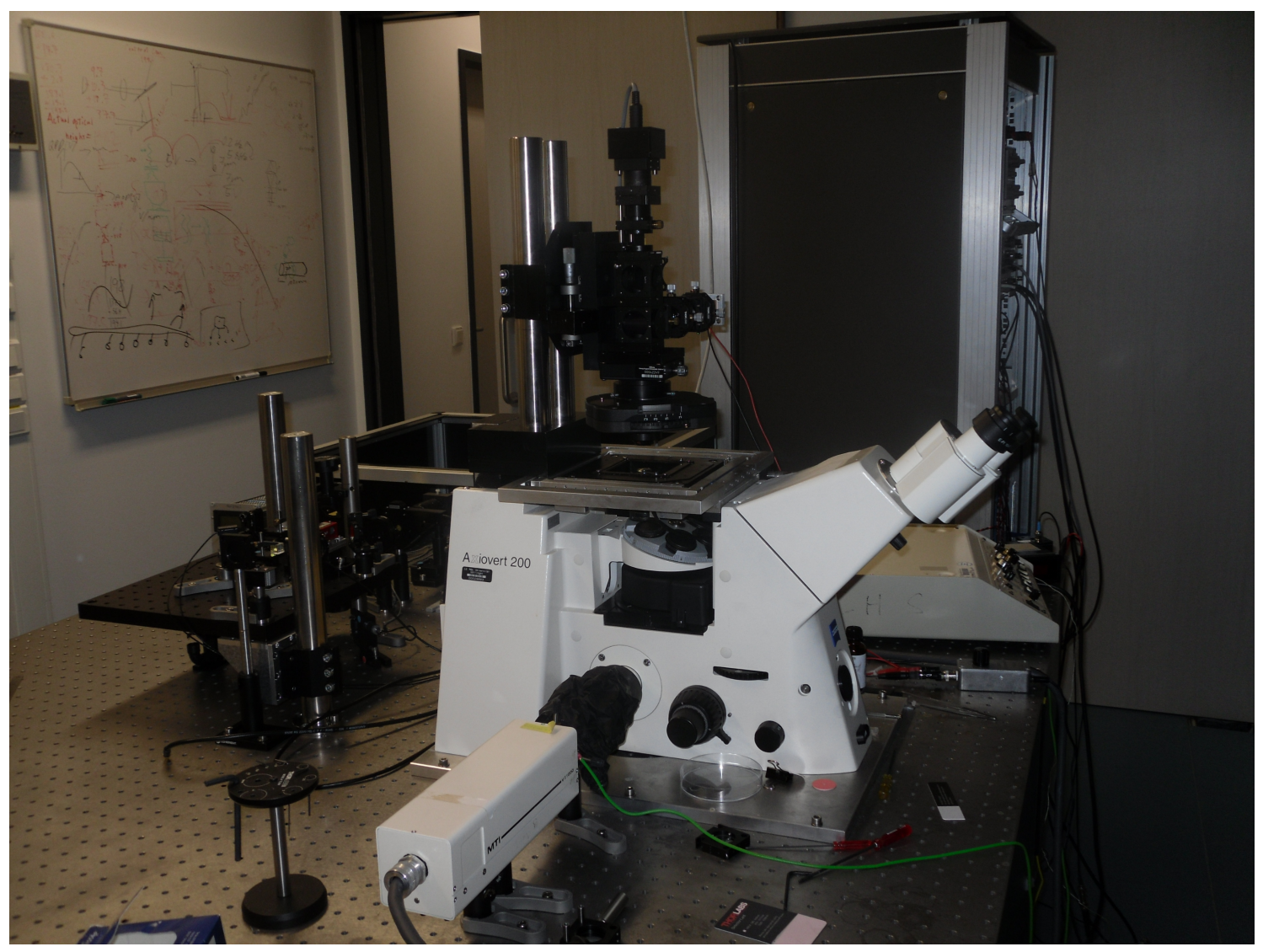

Figure 3.4: Photo of the DIC/dual-optical trap setup.

\section{Combined DIC/dual-optical trap microscope}

Figure 3.4 shows a photo of the setup. Most of the optics for the trapping beam sit on the optical table, whereas the illumination light source and its optics, as well as the trapping laser detection system, are mounted on a modified Zeiss microscope body. Two traps are created by splitting the IR laser light, intially polarized at $45^{\circ}$, into $s$ - and $p$-polarized components (with respect to the front edge of the microscope stage/first dichroic mirror DM1). These two traps are then steered separately and detected separately, giving us "two-for-one" trapping with a single laser. The disadvantage of this scheme apropos DIC is the aforementioned tradeoff between trapping quality and image quality, due to depolarization effects, if these effects are uncompensated. Figure 3.5 shows a schematic of the setup. Since a basic review of optical trapping and descriptions of the relevant components are provided in Chapter 2, we simply describe the setup for completeness and then concentrate on the modifications necessary for high-quality DIC. Starting with the trapping laser, the components of the setup are as follows: the trapping laser is a linearly polarized 


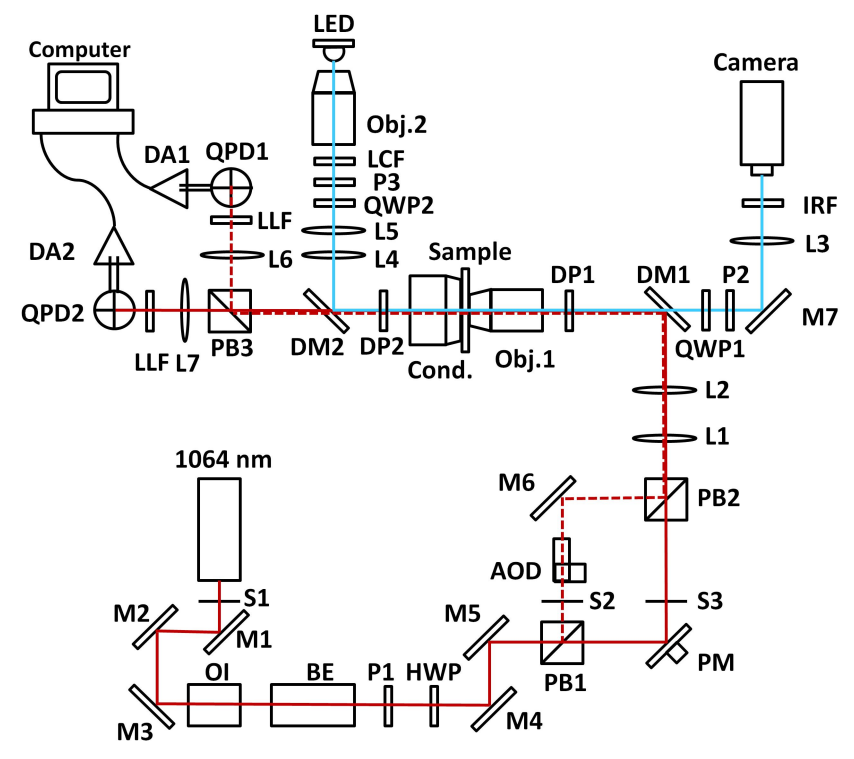

Figure 3.5: Schematic of the experimental setup described in the text. Red solid and dotted lines denote the IR laser beam paths. The blue solid line denotes the illumination light path. S1-S3 indicate shutters in the beam line, which are not included in the description in the text.

near-infrared laser $(1064 \mathrm{~nm})$ protected against back-reflections by an optical isolator $(\mathrm{OI})$. We expand the beam to $\sim 6 \mathrm{~mm}$ with an adjustable beam expander (BE) in order to increase trapping efficiency $[31,32]$. A combination of $\lambda / 2$ waveplate (HWP) and Glan-laser polarizer (P1) is used to adjust beam intensity so that the laser can be operated at a constant power of $\sim 2 \mathrm{~W}$ for maximum stability. The beam height is raised above the optical bench with a two-mirror periscope (M4, M5) to couple it into a modified, inverted microscope body (Zeiss Axiovert 200). The laser light is then split into orthogonally polarized $s$ - and $p$-components with a polarizing beam splitter (PB1) to create two traps. The $p$-polarized beam is steered with a piezo mirror $(\mathrm{PM})$ while the $s$-polarized beam is steered with an acousto-optic deflector (AOD). Both beams are recombined with a second, identical polarizing beam-splitter (PB2) and directed into the microscope body via a pair of telescope lenses $(\mathrm{L} 1, \mathrm{~L} 2)(\mathrm{f}=150 \mathrm{~mm}, \mathrm{f}=200 \mathrm{~mm})$. A dichroic mirror (DM1) couples the incoming laser light into the microscope light path and through a DIC prism into an oil-immersion, infinity-corrected objective (Obj.1) which forms two optical traps in the sample plane. Importantly, the DIC prism is rotated by $45^{\circ}$ from the orientation it normally has in the Zeiss Axiovert, so that the shear direction of the prism is orthogonal to the plane of incidence of DM1. This geometry guarantees that the two laser beams are not split again, but are only slightly shifted apart. Most noticeably, the reorientation of the DIC prism prevents cross-talk between the 
two traps (see Appendix B). The sample is mounted on an $\mathrm{x}-\mathrm{y}-\mathrm{z}$ piezo stage for nanometer-scale positioning. An oil-immersion condenser (Cond.) collects the laser light passing through the sample, which then goes through a second DIC prism to a second dichroic mirror (DM2). After passing straight through DM2 the laser light is split by a polarizing beam-splitter (PB3) into the two orthogonally-polarized components for the detection of displacements and forces in the respective traps. The condenser back-focal plane (BFP) is imaged onto two quadrant photodiodes (QPD1, QPD2) using lenses L6 and L7 $(\mathrm{f}=45 \mathrm{~mm})$. Laser-line filters (LLF1, LLF2) are placed in front of the diodes to block spurious signals from other light sources. Both photodiodes are operated at $160 \mathrm{~V}$ reverse bias to guarantee fast response at $1064 \mathrm{~nm}$ wavelength [58]. The QPD signals are amplified by low-noise, custom-built pre-amplifiers and differential amplifiers. Analog-to-digital conversion of the diode signal is done with a field-programmable gate array (FPGA) board, and the signal is recorded using custom-written LabView VIs (National Instruments, Austin, TX, USA).

Illumination light for DIC imaging is provided by a high-power blue light-emitting diode (LED) which is collimated with a 30x air objective (Obj.2). A laser clean-up filter (LCF), is placed after the objective to restrict the LED spectral bandwidth. The polarization of the illumination light needs to be oriented at $45^{\circ}$ to the shear axis of the DIC prism for proper DIC, and thereby at $45^{\circ}$ to the plane of incidence at DM1 and DM2. This also corresponds to an angle of $45^{\circ}$ to the front edge of the microscope stage. Polarization is set by a linear polarizer (P3). We then precompensate the depolarization the illumination light suffers upon reflection from the dichroic (DM2) with a $\lambda / 4$ waveplate (QWP2). A telescope lens pair (L4, L5) (f $=35 \mathrm{~mm}, \mathrm{f}=30 \mathrm{~mm}$ ) images the light source into the condenser BFP to achieve Köhler illumination, where it is sheared into two orthogonally polarized components by the DIC prism (DP2). The sheared beams are recombined after the objective by the second DIC prism (DP1) and then pass through DM1, QWP1 and the analyzer (P2). QWP1 served to correct depolarization caused by passage through DM1. A final lens (L3) images the illumination light onto an analog camera which is read out with a frame-grabber and a custom-written LabView VI. Table 3.1, at the end of this chapter, tabulates a list of relevant parts and their manufacturers.

\section{Depolarization effects in the setup and how to cor- rect them}

Light that is not entirely $s$ - or $p$-polarized at an oblique incidence on a multilayered thin-film structure such as a dichroic mirror undergoes two changes which are detrimental to DIC imaging: a relative phase shift between $s$ - and $p$-polarized 
components and an optical rotation, both of which are wavelength dependent ${ }^{1}[42]$. Both effects at their heart are due to the different boundary conditions (BCs) that an electromagnetic wave of $s$ - or $p$-polarization must satisfy when incident upon a discontinuous dielectric boundary. The optical rotation is evident given the Fresnel transmission/reflection coefficients, which themselves can be derived from Maxwell's equations and the appropriate BCs (e.g. that the tangential component of the electric field/normal component of magnetic induction is continuous across the boundary):

$$
\begin{aligned}
t_{\|} & =\frac{2 n_{1} \cos \theta_{1}}{n_{2} \cos \theta_{1}+n_{1} \cos \theta_{2}} \\
t_{\perp} & =\frac{2 n_{1} \cos \theta_{1}}{n_{1} \cos \theta_{1}+n_{2} \cos \theta_{2}} \\
r_{\|} & =\frac{n_{2} \cos \theta_{1}-n_{1} \cos \theta_{2}}{n_{2} \cos \theta_{1}+n_{1} \cos \theta_{2}} \\
r_{\perp} & =\frac{n_{1} \cos \theta_{1}-n_{2} \cos \theta_{2}}{n_{1} \cos \theta_{1}+n_{2} \cos \theta_{2}}
\end{aligned}
$$

where $t_{\|} / t_{\perp}$ are the parallel/perpendicular ( $p$ and $s$, respectively) components of the transmitted electric field vector, $r_{\|} / r_{\perp}$ are the parallel/perpendicular components of the reflected electric field vector, $n_{1}$ is the refractive index of the half-space of incidence, $n_{2}$ is the refractive index of the half-space of transmission, $\theta_{1}$ is the angle of incidence, and $\theta_{2}$ is the angle of the transmitted wave with respect to the vertical in the half-space of transmission. The two angles, $\theta_{1}$ and $\theta_{2}$, are related by the familiar law of refraction

$$
n_{1} \sin \theta_{1}=n_{2} \sin \theta_{2}
$$

Since $\theta_{1}$ and $\theta_{2}$ are not equal if there is a dielectric boundary, the transmission and reflection coefficients are in general different for $s$ - and $p$-polarizations. Since the final polarization state is given by a vector sum of the two orthogonal components, even in cases with no phase shift the difference in the reflection/transmission coefficients implies a change in the relative amplitudes of the reflected/transmitted field vectors, leading to an optical rotation.

To understand the wavelength dependence of the rotation and the phase shift, we have to dig deeper into the phenomena of reflection from/transmission through a stack of multiple dielectric interfaces, as is the case in dichroic mirrors. Classically these mirrors are made of alternating layers of high/low refractive index material, such as $\mathrm{ZnS}$ and $\mathrm{YF}_{3}$, of a quarter-wave thickness for a given design wavelength [63].

\footnotetext{
${ }^{1}$ In the following section we follow the treatment given in Reference [42]
} 


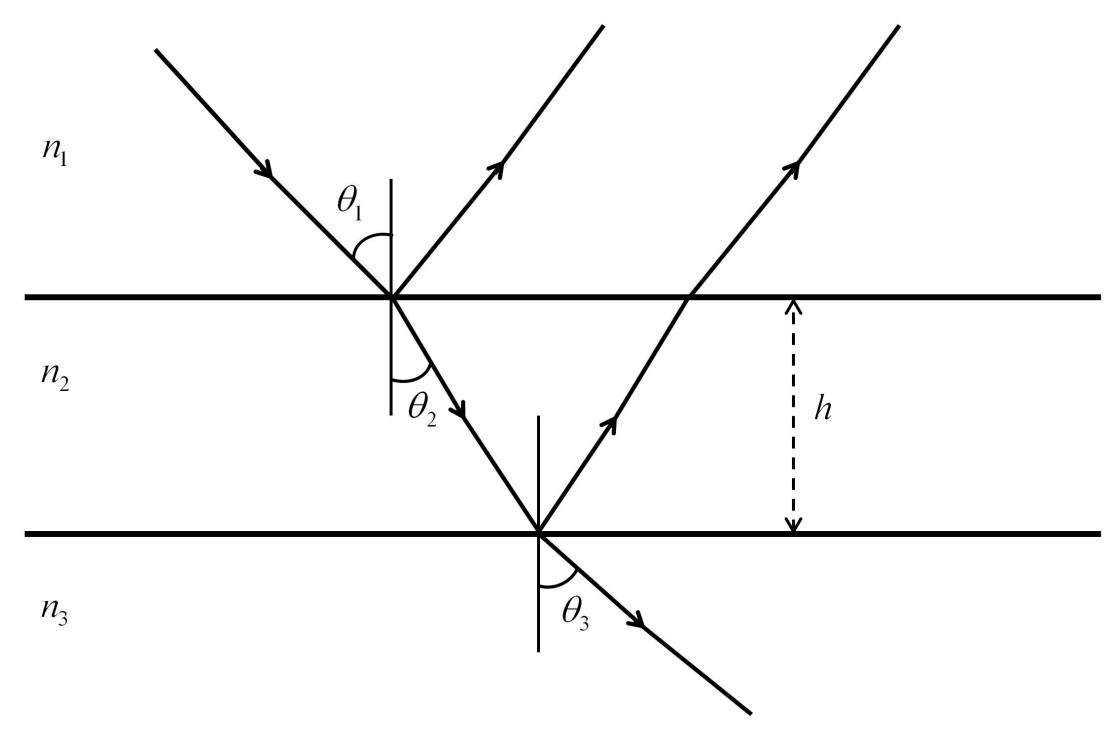

Figure 3.6: Schematic of a dielectric slab with refractive index $n_{2}$ sandwiched between two half-spaces of refractive index $n_{1}$ and $n_{3}$. The incident ray is reflected/refracted at each boundary with angles $\theta_{1}, \theta_{2}$, and $\theta_{3}$, according to the laws of reflection and refraction.

In this geometry the total reflected/transmitted beam is made up of a sum of multiple reflected/transmitted beams from the successive interfaces, giving rise to a series of first order differential equations and their respective BCs. These can be solved by the matrix method introduced by Abelés [42]. As a simple example, we consider the case of reflection from a single homogenous dielectric slab sandwiched between two half-spaces, as illustrated in Figure 3.6. Using the matrix formalism and treatment in Ref. [42] and the aforementioned Fresnel coefficients for reflection, the final reflection coefficient can be written as a combination of the reflections from the top and bottom of the slab, and is given by

$$
\begin{aligned}
r_{\|} & =\frac{r_{\|}^{12}+r_{\|}^{23} e^{2 i \beta}}{1+r_{\|}^{12} r_{\|}^{23} e^{2 i \beta}} \\
r_{\perp} & =\frac{r_{\perp}^{12}+r_{\perp}^{23} e^{2 i \beta}}{1+r_{\perp}^{12} r_{\perp}^{23} e^{2 i \beta}}
\end{aligned}
$$

with 


$$
\begin{aligned}
r_{\|}^{i j} & =\frac{p_{i}-p_{j}}{p_{i}+p_{j}} \\
r_{\perp}^{i j} & =\frac{q_{i}-q_{j}}{q_{i}+q_{j}} \\
p_{i} & =n_{i} \cos \theta_{i} \\
q_{i} & =\frac{\cos \theta_{i}}{n_{i}} \\
\beta & =\frac{2 \pi}{\lambda_{0}} n_{2} h \cos \theta_{2}
\end{aligned}
$$

where $r_{\|}^{i j}$ is the reflection coefficient of the electric field vector at the $i j$ interface, $r_{\perp}^{i j}$ is the reflection coefficient of the magnetic field vector at the $i j$ interface, $n_{i}$ is the refractive index of the $i$-th slab/half-space, $\theta_{i}$ is the corresponding angle of refraction/reflection, $h$ is the thickness of the dielectric slab, and $\lambda_{0}$ is the vacuum wavelength of the incident light. The phase shift, $\delta_{\| / \perp}$, of the parallel/perpindicular reflected beam is then given by

$$
\begin{aligned}
\tan \delta_{\|} & =\tan \left(\arg r_{\|}\right)=\frac{r_{\|}^{23}\left(1-\left(r_{\|}^{12}\right)^{2}\right) \sin 2 \beta}{r_{\|}^{12}\left(1+\left(r_{\|}^{23}\right)^{2}\right)+r_{\|}^{23}\left(1+\left(r_{\|}^{12}\right)^{2}\right) \cos 2 \beta} \\
\tan \delta_{\perp}=\tan \left(\arg r_{\perp}\right) & =\frac{r_{\perp}^{23}\left(1-\left(r_{\perp}^{12}\right)^{2}\right) \sin 2 \beta}{r_{\perp}^{12}\left(1+\left(r_{\perp}^{23}\right)^{2}\right)+r_{\perp}^{23}\left(1+\left(r_{\perp}^{12}\right)^{2}\right) \cos 2 \beta}
\end{aligned}
$$

From Eqns. 3.10 and 3.11 one can see that the phase of the two reflected waves is in general not the same ${ }^{2}$, as in general $r_{\|}^{i j} \neq r_{\perp}^{i j}$. The wavelength-dependence of the phase shift and optical rotation is evident in the dependence of $\beta$ on the vacuum wavelength $\lambda_{0}$. The optical thickness for which the reflection coefficient has maxima and minima, is given by

$$
H=\frac{m \lambda_{0}}{4 \cos \theta_{2}}, \quad(m=1,2,3, \ldots)
$$

Eqn. 3.12 implies that a given slab thickness only gives a maximum or minimum for a particular wavelength. In a multiple dielectric stack one has many different reflected waves which sum up and interfere according to their wavelength-dependent relative phase shifts, leading to differing optical rotations for different wavelengths.

In most commercial DIC microscopes, the illumination light is polarized parallel or perpendicular to the plane of incidence of dichroic mirrors that might be inserted

\footnotetext{
${ }^{2}$ Technically $\delta_{\|}$is the phase of the magnetic field vector, but as it represents a traveling electromagnetic wave in air, the phase of the electric field vector is identical
} 


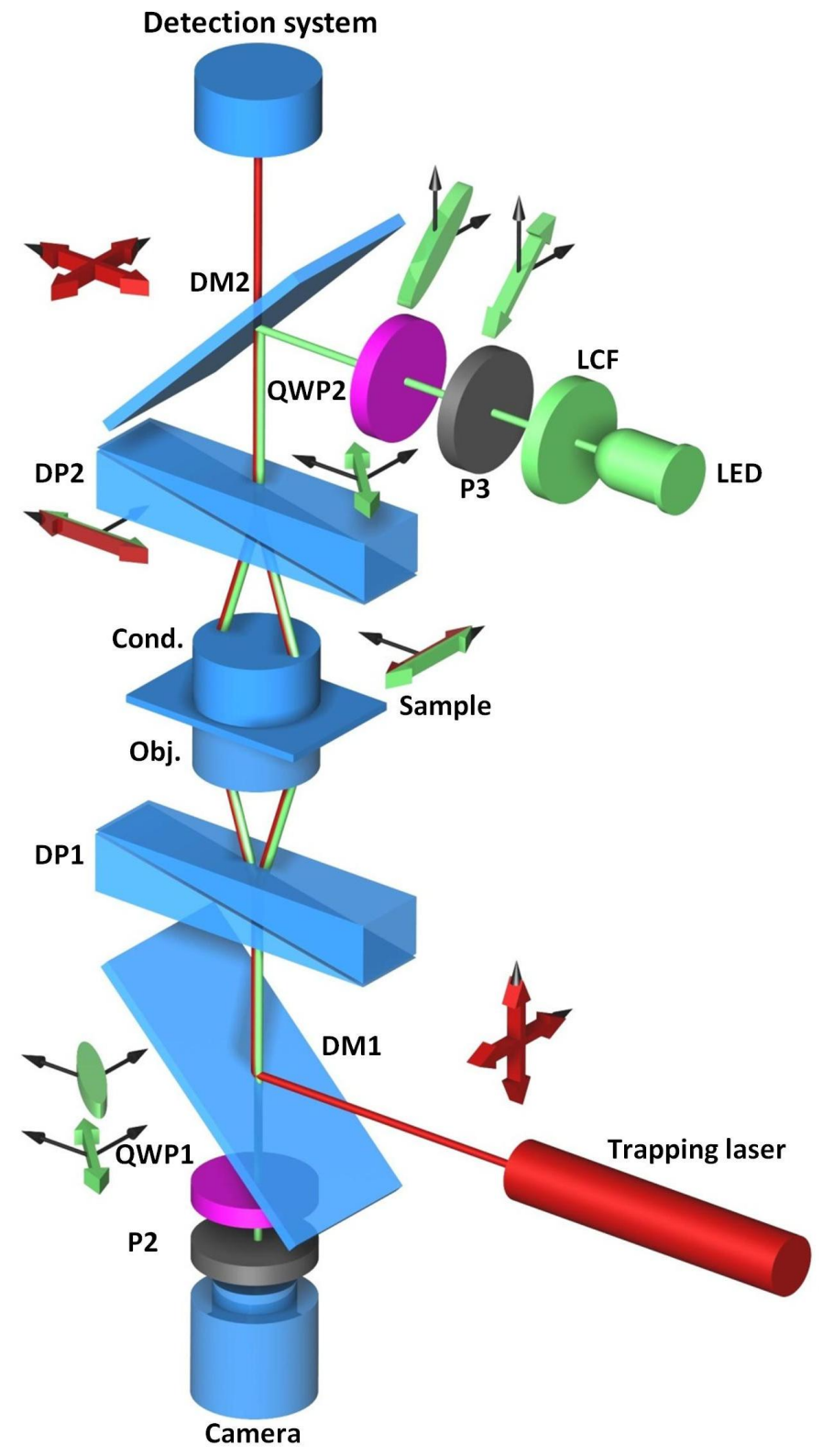

Figure 3.7: Schematic of illumination and optical trap pathways with polarization states. Green corresponds to the illumination light and red corresponds to the trapping laser. Double-headed arrows/ellipses show the polarization state at various points in the illumination pathway, and black arrows correspond to $s$ - and $p$-polarization axes with respect to the dichroic mirrors. Abbreviations: LED $=$ light-emitting diode, $\mathrm{LCF}=$ laser clean-up filter, $\mathrm{P}=$ polarizer, $\mathrm{QWP}=$ quarterwave plate, $\mathrm{DM}=$ dichroic mirror, $\mathrm{DP}=\mathrm{DIC}$ prism, Cond. = condenser, Obj. = objective. Drawing by Christoph F. Schmidt 
in the beam path, which often also means parallel or perpendicular to the front of the sample stage. The DIC prisms are then oriented at $45^{\circ}$ to the polarization vector. This geometry is what causes the problem in a dual-optical trap set-up, however, as it would lead to the aforementioned further splitting of the trapping beams if they came in $s$ - and $p$-polarized with respect to the dichroic mirror. To avoid this problem, we rotated the prisms in our microscope so that the shear axis is parallel/perpendicular to the polarization vectors of the two trapping laser beams. The illumination light then needs to be linearly polarized at $45^{\circ}$ to the prism axes, so that only the illumination light is split (see Figure 3.7). Unfortunately, this leads to the phase shift/optical rotation just discussed, and the light which was initially polarized at $45^{\circ}$ becomes elliptically polarized upon reflection from/transmission through the dichroics used to couple the illumination or laser light into/out of the beam path. Figure 3.8 shows the calculated change in ellipticity ${ }^{3}$ and rotation of the polarization axis after reflection from DM2 as a function of wavelength, following the conventions found in Ref. [42]. Data regarding reflectance and phase shift for $s$ - and $p$-polarizations upon reflection were kindly provided by the manufacturer (Chroma Technologies). Elliptical polarization leads to imperfect extinction at the analyzer and the spectral width of the light source leads to a spread in optical rotations/phase shifts. Although an LED light source has a relatively narrow band width (in our case: $425-94 \mathrm{~nm} 1 / e^{2}$ width), the spread in polarization state rotations noticeably decreases extinction and contributes to reduced image contrast.

To restrict the spectral spread in optical rotations, we inserted a laser cleanup filter (LCF) with a central wavelength of $467 \mathrm{~nm}$ and a $10 \mathrm{~nm}$ spectral width. To correct for the depolarization effects, we included two zero-order quarter wave plates (QWP1 \& QWP2) with a central wavelength of $470 \mathrm{~nm}$ into the illumination beam path, before and after DM2 and DM1 as shown in Fig. 3.7. The wave plates were rotated to pre- and post-compensate for the elliptical polarization caused by the dichroics. It should be noted that there are redundancies in the elliptical polarization degree of freedom in our setup, due to the DP1 prism. The shear axis of the prism can be translated in the BFP of the objective, leading to a relative phase shift between the $s$ - and $p$-polarized light. Thus, by leaving out the QWPs and running the prism to the end of its screw thread, one can recover the characteristic DIC extinction cross in the BFP when viewed with a Bertrand lens. Alternatively, since in our case the dominant wavelength passing through the LCF is $\sim 467 \mathrm{~nm}$ which is not strongly elliptically polarized but undergoes a large optical rotation (Fig. 3.8), one can rotate the polarizer by $90^{\circ}$ and again translate DP1 by a smaller amount to recover DIC extinction. Given this redundancy, the QWPs aren't strictly necessary to recover image quality, and they have the disadvantage of adding more elements to the optical path. However, as in some cases the ellipticity is more

\footnotetext{
${ }^{3}$ Positive/negative values of $\tan \chi$ denote right-handed/left-handed polarization states.
} 


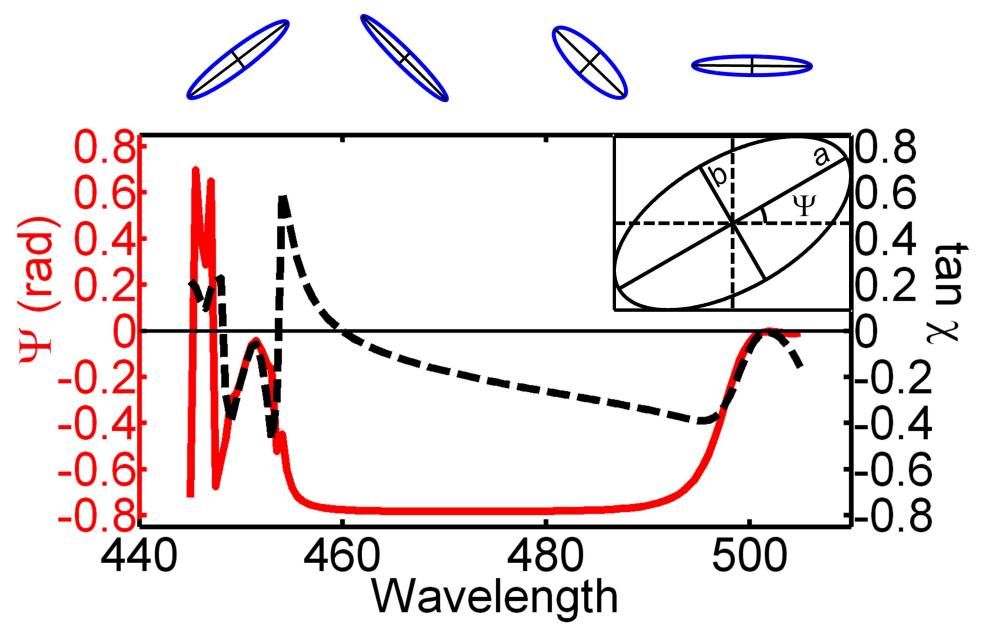

Figure 3.8: Calculated change of polarization state after reflection from DM2 as a function of wavelength. $\Psi$ (red solid line) gives the counterclockwise rotation with respect to the abscissa and tan $\chi=\mathrm{b} / \mathrm{a}$ (dotted black line) gives the ratio of the semi-minor axis to the semi-major axis (ellipticity). The inset shows the coordinate system with respect to the three variables. The four ellipses above show the elliptical polarization state and rotation after reflection at several wavelengths; left to right: 447, 467, 488, and $505 \mathrm{~nm}$.

pronounced (as in the range between 450-460 $\mathrm{nm}$ in Fig. 3.8) and correction of it more important, addition of QWPs gives the user added flexibility, similar to a de Sénarmont compensator. In general, the amount of depolarization will depend on the specific wavelength/dichroic mirror set combination, and the addition of QWP elements allows for modularity and ease of correction. To test the effect on the image quality of the aforementioned depolarization effects, we visualized microtubules fixed to a cover slip. Microtubules make an excellent test sample for the quality of DIC because, at a width of $\sim 25 \mathrm{~nm}$, they are below the resolution limit and thus invisible to normal brightfield microscopy. They can, however, be resolved with appropriately aligned DIC [64, 65]. To visualize microtubules, we constructed sample chambers by attaching a glass coverslip with two narrow strips of double-stick tape to a glass microscope slide, resulting in chambers with $\sim 80 \mu \mathrm{m}$ inner height. Coverslips were coated with trimethoxysilylpropyldiethylenetriamine (DETA) so that microtubules would adhere to them. Microtubules were polymerized in vitro from tissue-purified tubulin (porcine brain) following established recipes [66] and stabilized by adding $10 \mu \mathrm{M}$ taxol to BRB80 buffer consisting of $80 \mathrm{mM}$ PIPES, $1 \mathrm{mM}$ EGTA, \& $1 \mathrm{mM} \mathrm{MgCl}$ with $\mathrm{pH}$ 6.9. We filled sample chambers with $20 \mu \mathrm{l}$ of microtubule-containing BRB80, and imaged the microtubules that attached to the coverslip. All experiments were performed at room temperature. The results can be 

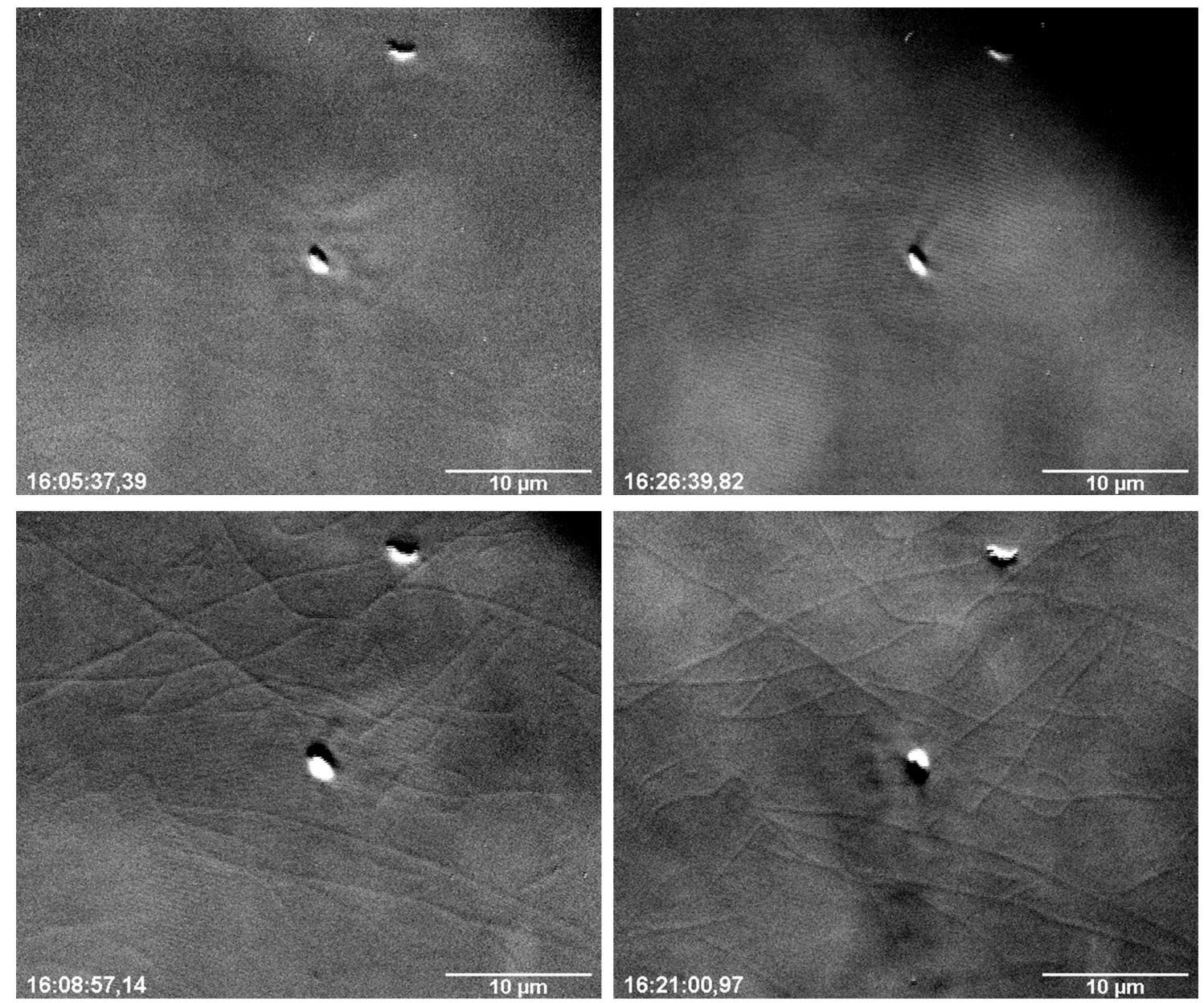

Figure 3.9: DIC images of microtubules fixed to a cover slip with varying degrees of compensation for the described polarization and optical rotation effects. From left to right, top to bottom: No compensation, only QWPs included, only LCF included, both QWPs and LCF included. All images are enhanced by background subtraction and represent the average of 10 frames at a video rate of $25 \mathrm{~Hz}$. Contrast was adjusted in each image individually for optimal visibility of the microtubules. 
seen in Figure 3.9 where the successive images are taken with (i) no compensation, (ii) with only the QWPs included in the beam path, (iii) with only the LCF included, and (iv) with both QWPs and the LCF included. As predicted in Fig. 3.8, the QWPs correct for the small elliptical polarization upon reflection from DM2, which can be seen in the characteristic DIC extinction cross (data not shown), but they provide little improvement in image quality. Adding the LCF, however, results a huge improvement, allowing resolution of microtubules which were previously invisible in the uncompensated image. This implicates the wavelength-dependent optical rotation as the main culprit in image degradation.

\section{Discussion}

The recovery of DIC quality shown in Fig. 3.9 shows how vital keeping a proper polarization state and restricting optical rotations is for DIC image formation. As image analysis is key to our study of cilium mechanics/dynamics, the quality of image formation, in particular image edges, is crucial if one hopes to extract meaningful information from a movie. This will be the topic of the next chapter, where we describe the algorithm we used to track cilia dynamics. The main drawback of DIC in comparison with fluorescence is its lower signal-to-noise ratio (SNR), which is typically a factor of four worse [67]. This lower SNR also requires more complicated image filtering to track features in micrographs. The label-free nature of DIC, however, allows one to see all cilia in the microscope's field of view, not just the sub-population of cilia from cells expressing a fluorescently labeled target protein. This turned out to be an advantage in our edge-viewing geometry, as it increased the proportion of visible cilia in any given sample. Indeed, when we were finally

able to produce a stably fluorecent cilia line, the lower proportion of labeled cilia made it hard to find trackable cilia on the edge that we visualized, and the SNR also wasn't dramatically better, due to background fluoresence from the cell. All in all, high-quality DIC was necessary for the tracking experiments that make up the main results of this work. 
Table 3.1: List of Setup Components

\begin{tabular}{|c|c|c|}
\hline Component & Part Name/Notes & Manufacturer \\
\hline NIR laser & Compass 4000 & Coherent Inc., Santa Clara, CA, USA \\
\hline$(1064 \mathrm{~nm})$ & $\mathrm{cw}, \max$ power $4 \mathrm{~W}$ & \\
\hline $\mathrm{OI}$ & IO-3-1064-VHP & Optics for Research, Caldwell, NJ, USA \\
\hline $\mathrm{BE}$ & $4401-359-000-20$ & Qioptiq Photonics, Luxembourg \\
\hline HWP & WPH05M-1064 & Thorlabs Inc., Newton, NJ, USA \\
\hline $\mathrm{P} 1$ & PB-15B-1064 & BFi Optilas, Evry, France \\
\hline $\mathrm{P} 2, \mathrm{P} 3$ & NT47-216 & Edmund Optics Ltd., York, UK \\
\hline M1, M2 & BB1-E03 & Thorlabs Inc., Newton, NJ, USA \\
\hline $\mathrm{M} 3, \mathrm{M} 4, \mathrm{M} 5, \mathrm{M} 6$ & M01 & Thorlabs Inc., Newton, NJ, USA \\
\hline $\mathrm{PB} 1, \mathrm{~PB} 2$ & PBS-1064-040 & BFi Optilas, Evry, France \\
\hline $\mathrm{PM}$ & AG M100 & Newport Co., Irvine, CA, USA \\
\hline AOD & DTSXY-400-1064 & AA Optoelectronic, Orsay Cedex, France \\
\hline L1, L2, L4, L5, L6, L7 & achromatic doublets & Thorlabs Inc., Newton, NJ, USA \\
\hline L3 & 444034 10x/25 Br. foc. eyepiece & Carl Zeiss AG, Jena, Germany \\
\hline DM1 & $725 \mathrm{dcspxr}$ & Chroma Technologies, Bellows Falls, VT, USA \\
\hline Obj.1 & $\begin{array}{c}\text { Plan-Neofluar } 440480 \\
\text { 100x, NA } 1.3\end{array}$ & Carl Zeiss AG, Jena, Germany \\
\hline Obj.2 & $30 \mathrm{x}, \mathrm{NA} 0.65$ & Rolyn Optics Co., Covina, CA, USA \\
\hline Piezo stage & $\mathrm{P}-561.3 \mathrm{CD}$ & $\begin{array}{c}\text { Physik Instrumente GmbH, Karlsruhe, } \\
\text { Germany }\end{array}$ \\
\hline Cond. & 424208, NA 1.4 & Carl Zeiss AG, Jena, Germany \\
\hline DM2 & z488rdc & Chroma Technologies, Bellows Falls, VT, USA \\
\hline PB3 & G335-728-000 & Qioptiq Photonics, Luxembourg \\
\hline QPD1, QPD2 & SPOT-9-YAG & OSI Optoelectronics, Hawthorne, CA, USA \\
\hline LLF1, LLF2 & FL 1064-3 & Thorlabs Inc., Newton, NJ, USA \\
\hline FPGA & PCI-7833R & National Instruments, Austin, TX, USA \\
\hline LED & $\begin{array}{c}\text { Ostar LE-B-Q9WP } \\
456 \mathrm{~nm} \text { peak } \lambda, 1-1.6 \mathrm{~W}\end{array}$ & $\begin{array}{l}\text { OSRAM Opto Semiconductors GmbH, } \\
\text { Regensburg, Germany }\end{array}$ \\
\hline $\mathrm{LCF}$ & $\mathrm{z} 467 / 10$ & AHF Analysetechnik, Tuebingen, Germany \\
\hline QWP1, QWP2 & CM 470 & Comar Instruments, Cambridge, UK \\
\hline DP1, DP2 & & Carl Zeiss AG, Jena, Germany \\
\hline Camera & MTI VE1000 & DAGE-MTI, Michigan City, IN, USA \\
\hline Frame grabber & PCI-1405 & National Instruments, Austin, TX, USA \\
\hline
\end{tabular}




\section{Chapter 4}

\section{Tracking DIC line images}

The human eye is a wonderfully evolved tracking instrument. It can detect brightness levels ranging over 10 decades and its rod cells can reportedly detect single photons. Additionally a vast, poorly understood machinery of image processing takes place, both in the retina and in the brain, which allows humans to detect colors, edges, perform contrast enhancement, segment images, average noise, and various other signal processing tasks [65]. This means that we can easily detect and pick out features of interest, especially moving ones, almost effortlessly while machine vision algorithms struggle to keep up. When faced with tens of thousands of frames of data to sift through, however, we quail and leave the algorithms to do our dirty work for us. In the following we describe a Matlab (Mathworks, 2009 release) routine written to track DIC line images of cilia. The general idea comes from a paper by Danuser et al. [68], though we implement it somewhat differently than they do. We first describe the general problem of extracting a line object from a DIC image and how we solve it, followed by a discussion of the performance of our algorithm. We end with a brief description of the tracking and reconstruction technique we use to analyze the confocal stack data presented in the Results section.

\section{Extraction of a line object from a DIC image}

The left-hand picture in Figure 4.1 shows a typical micrograph of a cilium imaged with our DIC microscope. The eye can clearly make out the image of the cilium, despite the large intensity variations in the background, which are of the same order as the intensity variations across the cilium. The human visual system identifies line features via visual orientation maps, where certain groups of neurons in the visual cortex are preferentially activated for certain line segment orientations [69]. We aim to create a very simple "response map" for a particular feature, in this case a specific line width and orientation, through a series of filtering steps. The two main features of the line image in Fig. 4.1 are an intensity gradient variation in one dimension 

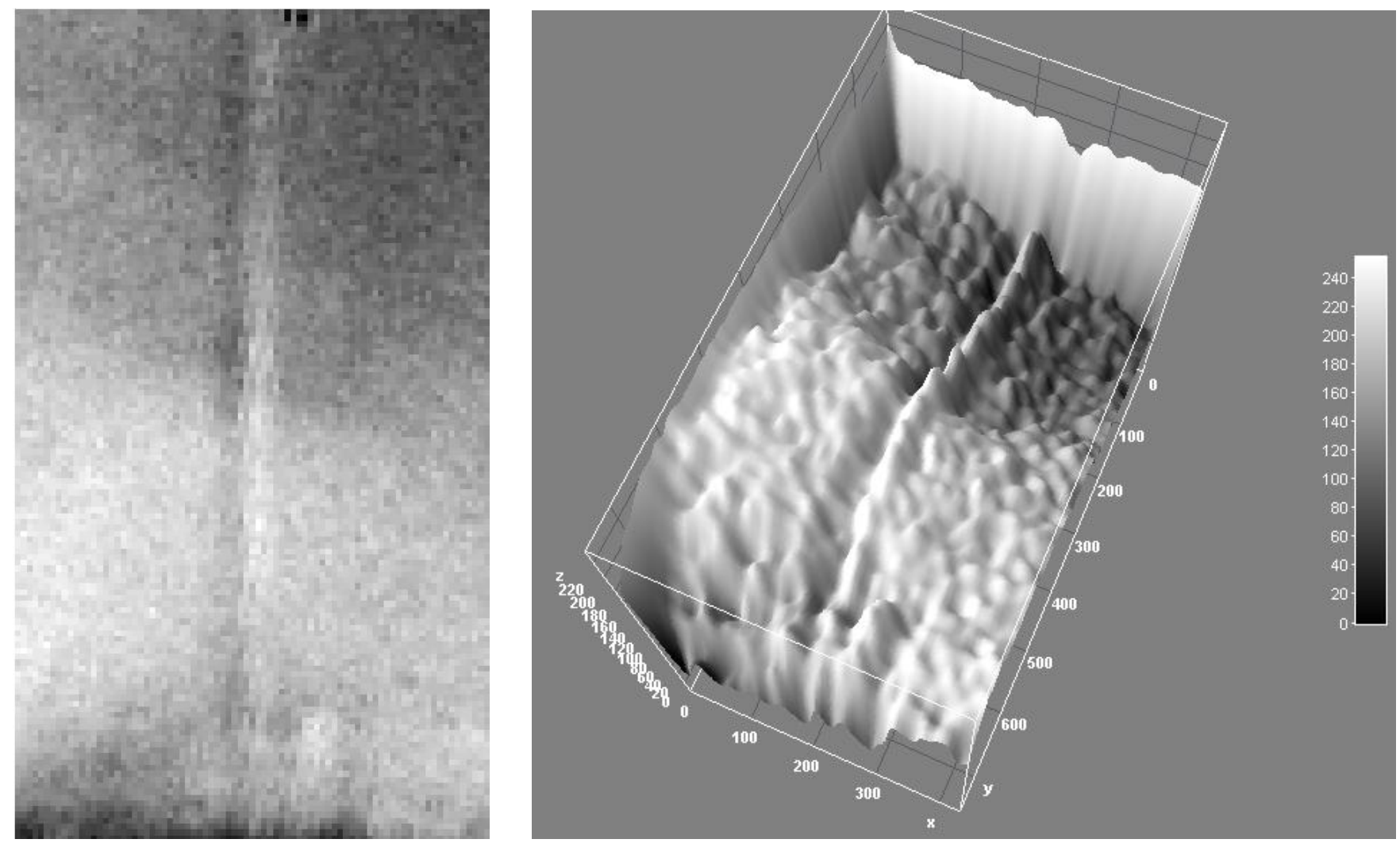

Figure 4.1: Micrograph of cilium imaged with DIC (left) and 3D representation (right) done with ImageJ 3D Viewer. The 3D representation highlights the fact that the line response (i.e. the valley followed by a peak) is similar across the image, despite the large background intensity variation.

(transverse to the cilium axis) and little or no variation along the other dimension (longitudinally along the cilium axis). The 3D rendering of the micrograph (righthand side, Fig 4.1) makes the line response more explicit: as one traverses the image transverse to the cilium one comes across a "valley and mountain" motif, arising from the first-derivative-like image formed by DIC. Despite the large intensity variation in the image, this feature is apparent against the background along the length of the cilium. It is knowledge of this characteristic feature that we'll use to pick out the line from the background. To enhance the line signal we go through three image processing steps, shown from left to right in Figure 4.2. The first two steps, Gaussian filtering and edge-filtering, can be thought of as parts of a larger edge detection strategy described by John Canny in a seminal 1986 paper [70]. In it Canny used numerical optimization techniques to find an optimal filter for a step-type edge that i) minimized erroneous/maximized real edge detection, ii) localized edges as 

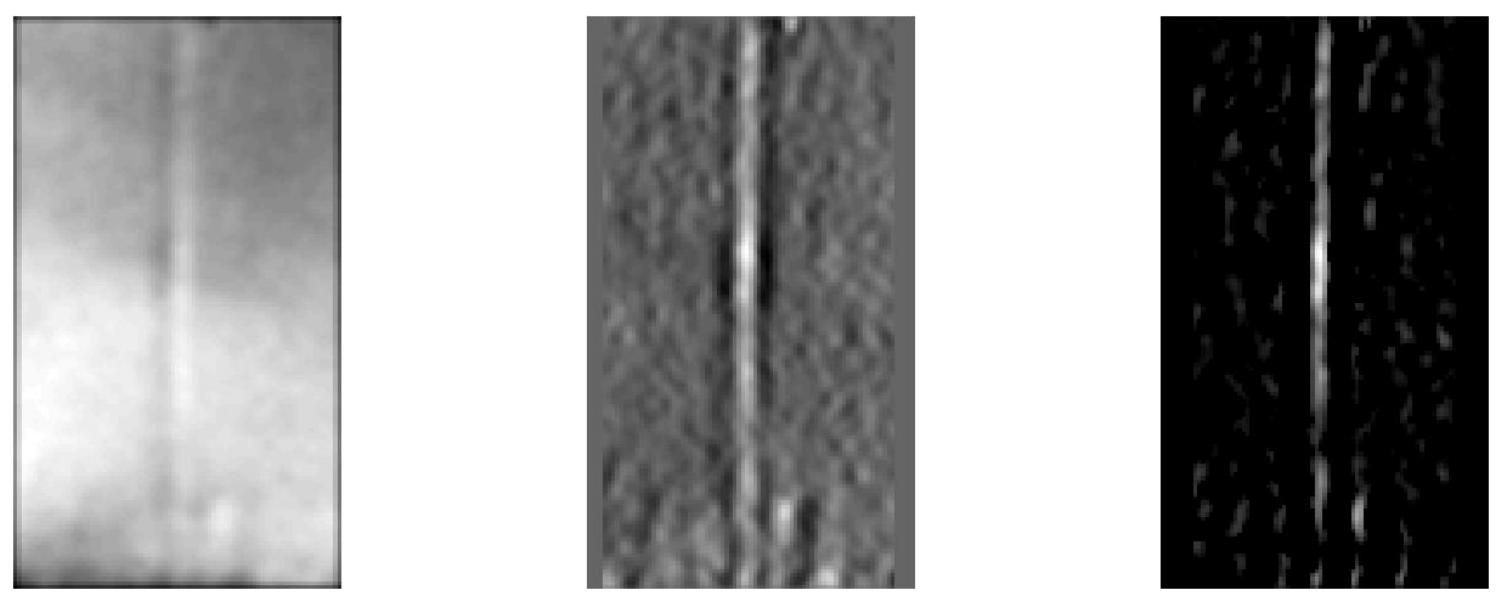

Figure 4.2: Image processing steps. From left to right: First, the image is Gaussianfiltered to smooth out noise. Second, the image is edge-filtered, to highlight the gradient changes. Third, it is filtered with the MGM filter described in the text, which enhances the features associated with DIC line images.

close as possible to their real location, and iii) marked detected edges only once. Interestingly, he showed that there is an inherent trade-off between criteria i) and ii), which he termed an uncertainty principle between the two. Uncertainty principles notwithstanding, he went on to find an optimal filter numerically and concluded that it was well-approximated by the (directional) derivative of a Gaussian, i.e.

$$
K_{o p t}(x) \approx \frac{-x}{\sigma^{2}} \exp \left(\frac{-x^{2}}{2 \sigma^{2}}\right)
$$

where $K_{\text {opt }}$ is an optimal filter and $\sigma$ is the Gaussian width. Following this strategy we convolve the original image with a Gaussian kernel, $G$, and then a 1D derivative kernel (in this case the Sobel operator), $D$, to get first the left and then the middle image in Fig. 4.2. Mathematically speaking (in 1D) we perform

$$
P_{2}(x)=D * P_{1}(x)=D *\left(G * P_{0}(x)\right)
$$

where $P_{0}$ is the original image, $P_{1}$ is the Gaussian convolved image, and $P_{2}$ is the derivative of a Gaussian convolved image ${ }^{1}$. Figure 4.3 shows plots of the intensity vs. transverse position for the midline of the image throughout the various image processing steps. The Canny filtering scheme serves to smooth out short length scale noise variations and enhance local gradient differences, as can be seen in the evolution of the profile in the first three graphs. After convolving our image with the derivative of a Gaussian, shown in the lower left-hand graph, the filtered image

\footnotetext{
${ }^{1}$ Note that, for the discrete difference operator $D, D(f * g)=(D f) * g=f *(D g)$.
} 

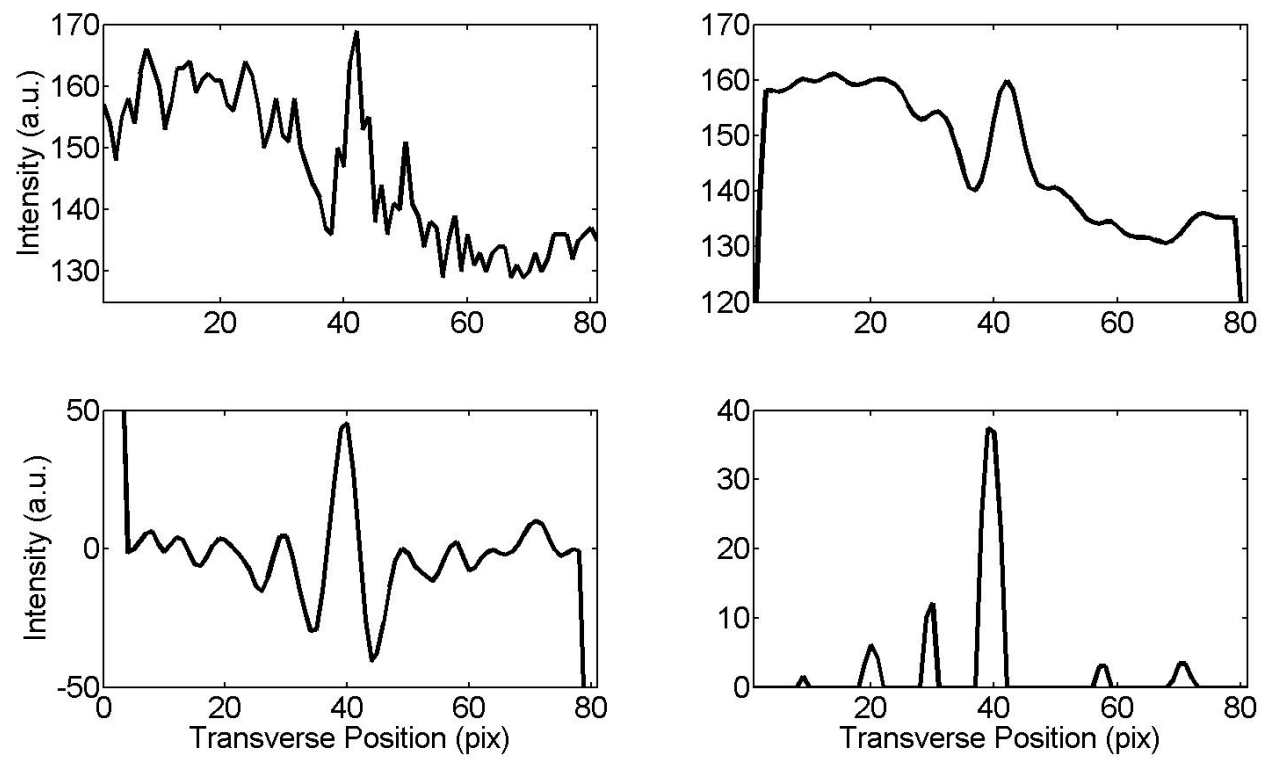

Figure 4.3: Mid-line transverse cross sections of the image over the image processing steps. The cilium centerline is at pixel 40 in the image (see Figure 4.1) as judged by eye. The top left graph shows the original image cross section, which has large noise variations and a large gradient from light to dark which makes extracting the cilium position difficult. Top right: After Gaussian-filtering, the short-scale noise variations are smoothed out, but the large gradient remains. The cilium peak close to pixel 40 is much clearer against the background. Bottom left: Edge-filtering takes the derivative of the image, smoothing out the large-scale gradient. Bottom right: The modified MGM filter produces a response map of line features, setting non-line features to zero. This further enhances the maximum associated with the cilium at pixel 40 in the edge-filtered image and reduces the remaining noise.

has a large maximum where the edge is located (pixel position 40) and two negative minima a distance $s$ away on either side. In order to further enhance the edge and separate it unambiguously from the background, we apply the nonlinear filtering approach described in Ref. [68]. We dub this final filter a "modified geometric mean" (MGM) filter and its effect is pictured in the final image in Fig. 4.2 as well as the final graph in Fig. 4.3. The MGM filter, $F_{M G M}$, performs the following operation

$$
F_{M G M}(x)=\sqrt[3]{[-I(x-s)]^{+}[I(x)]^{+}[-I(x+s)]^{+}}
$$

where 


$$
[x]^{+}= \begin{cases}0 & \text { if } x<0 \\ x & \text { if } x \geq 0\end{cases}
$$

and $I$ is the intensity while $s$ is the filter scale. In this way the MGM filter produces a response proportional to the geometric mean when it finds a positive intensity value with two negative values a distance $\pm s$ away and produces a null response if any of these conditions are violated. The distance $s$ from the maximum to the minima is not known a priori and it depends on the DIC image formation process, the size of the line object being imaged, and the Gaussian width $\sigma$ that the image is convolved with. To determine $s$, we filter the image with a series of different scales, ranging from 1-10 pixels. Examples of filter response maps for three different scales are shown in Figure 4.4. When the filter scale is too small, as in the leftmost picture, or too large, the line isn't found at all. At intermediate though non-optimal values, as in the rightmost picture in Fig. 4.4, most of the line is still visible but the response map is patchier than in the optimal filter case (middle picture). By comparing the total intensity of the response map versus filter scale (Fig. 4.4, bottom graph) we pick out the filter scale corresponding to the maximum response and use this value for $s$. This method for determining $s$ is vulnerable in principle to line noise at different scales, e.g. high spatial frequency camera line noise, but in practice it works well for our images. Although the width of the line can also change, getting broader when going slightly out of focus for example, in practice a filter scale of 4-5 pixels is usually the optimum, given a Gaussian width of $\sigma=1.5$ pixels, which is enough to smooth out high-frequency noise. It should be noted that the Sobel operator we use only takes the derivative in the direction transverse to the cilium, and we apply the MGM filter line-wise along the transverse direction. Taking additional directional derivatives only serves to add noise and since the cilium rarely rotates or bends more than $10^{\circ}$ away from its center position, 1D filtering suffices to track the cilium in all our cases.

Once all of the filtering steps have been completed, the cilium profile looks like that shown in the lower right-hand corner in Fig. 4.3. To pick out the cilium position, our algorithm performs a parabolic fit to the maximum of each transverse line profile. This yields the backbone seen in the red line overlay on the left-hand side of Figure 4.5. While the algorithm has successfully located the cilium backbone over most of the cilium length, it loses it at the very top and at the bottom of the cilium. One can see from the middle line response map pictured in Fig. 4.4 why this is; while the cilium is the unambiguous maximum over most of the image, near the base a second line appears to the right and the cilium disappears. At the very tip of the cilium a dark fleck of dirt on the screen creates a much larger line response than the lower contrast cilium tip and algorithm consequently fits that maximum. To mitigate this problem we imposed an outlier rejection subroutine in the algorithm: 
Filter Scale=3

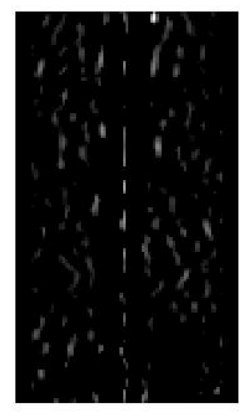

Filter Scale $=5$

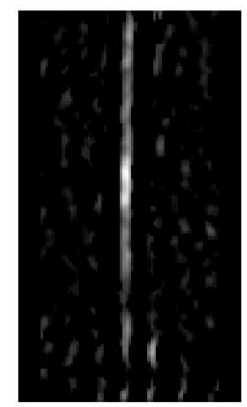

Filter Scale $=7$

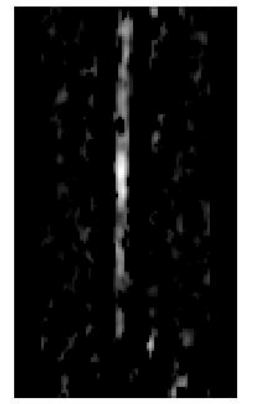

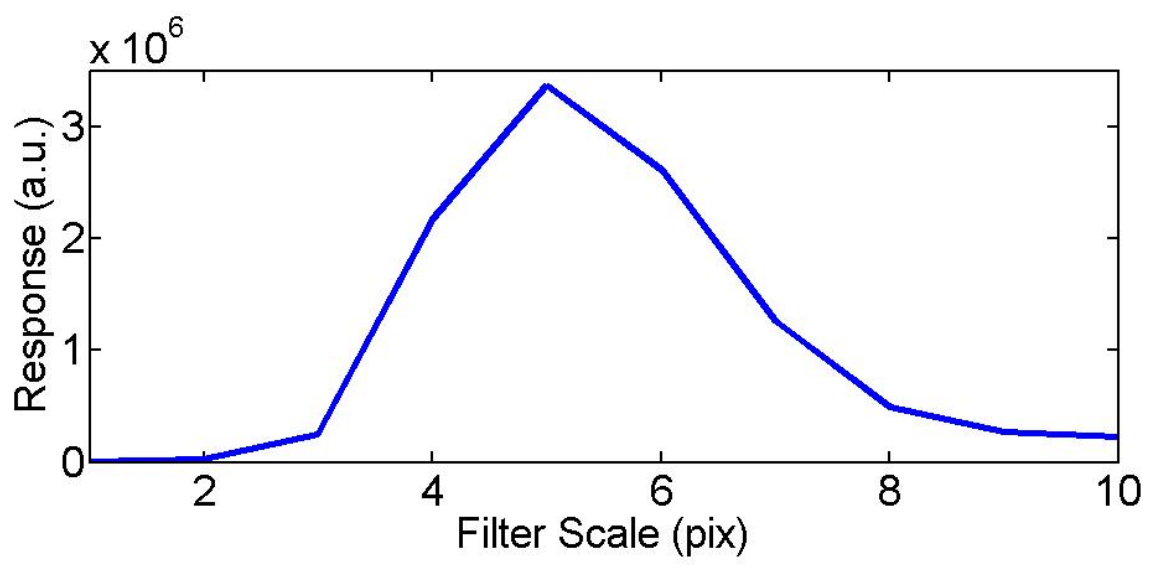

Figure 4.4: Comparison of various filter scales. Top row: Response maps after MGM filter scales of 3, 5, and 7 pixels. Bottom: Filter scale v. total intensity of the corresponding response map. One sees a clear maximum at a filter scale size of 5 pixels, agreeing with best line representation seen by eye.

1. At the beginning of the algorithm tracking process the user must provide an initial guess of the cilium position in the form of a hand-traced backbone overlay, preferably binarized.

2. Once the first backbone has been determined, the algorithm compares the values with the initial guess to see if any of them deviate from the initial guess by more than the filter scale, $s$. If they do, it replaces these backbone points with values linearly interpolated between non-replaced backbone points.

3. The algorithm proceeds forward to the next frame. Once the second backbone has been determined, step two is again performed, except that the second backbone is compared against the outlier-rejected first backbone values rather than the initial guess. 

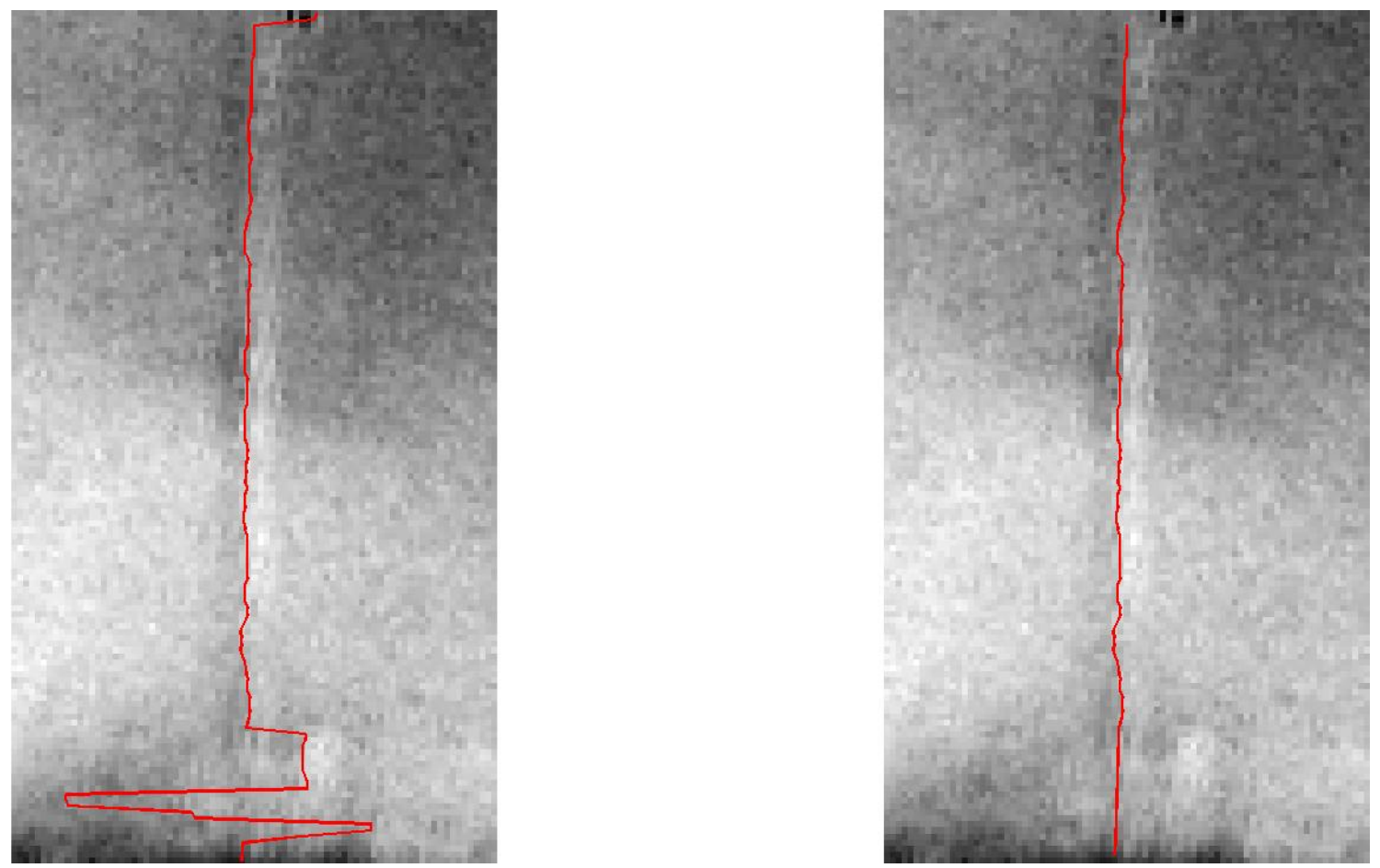

Figure 4.5: Rejection of outliers. Left: Uncorrected fit of a cilium backbone. Right: Backbone fit with outlier rejection routine described in the text.

4. For later frame iterations, the backbone is compared against the median value of the previous 2, 3, or 4 outlier-rejected backbones (always the last 4 for step 5 or higher), and values deviating more than a filter width away are linearly interpolated from non-rejected values.

The results of the outlier rejection scheme can be seen on the right-hand side of Fig. 4.5 (the red line overlay). The outlier-rejected backbone does a good job of fitting the cilium image that we see by eye. Choosing the filter scale as the maximum possible movement between frames was motivated by the observation that the cilium didn't move a distance greater than half the filter scale from frame-to-frame. Also, none of the cilia examined have sharp kinks and they have a persistence length far beyond their total length, rendering them mostly rigid. Thus there is never any case where one sees a cilium go through a sharp bend from one frame to another, or even between 4 frames, such that the filter scale constraint condition is violated. Finally the last step in the outlier rejection subroutine, where one takes the median of the last several frames, is implemented to allow the routine to avoid errors. Background particles occasionally move through the frame behind or in front of the cilium and can dominate the algorithm response, taking several frames to diffuse through. With the right diffusion rate they can bend the backbone gradually, not violating the filter 
scale condition from one frame to the next. A stricter, Markov-like condition which only looks back at the backbone position in the previous frame is liable to follow such particle noise for longer, whereas our condition, with its longer memory, is less likely to follow such deviations. The choice of four frames for the memory and not longer is to avoid impinging on actual cilium dynamics.

The observant reader may notice that the deviation at the tip in Fig. 4.5 has not been fitted, and the topmost value in the cilium backbone has been ignored. This is due to the fact that there is no uppermost value to interpolate to, and the value is assigned as not-a-number ( $\mathrm{NaN})$. The cilium tip and base are often hard to track, and thus commonly have ambiguous NaN values. For this reason we confine our tracking analysis to a middle range of the cilium and don't include the base and tip.

\section{Performance}

To test the performance of our algorithm, we put it through several trials. First, in order to measure its performance in optimal conditions, we recorded a movie of a cilium which had been shed from the cell sheet and had sedimented on the coverslip. The background tends to be more uniform at the coverslip than at the folded cell sheet edge, producing a clearer DIC image of the cilium. Figure 4.6 shows a micrograph of the cilium as well as a superimposed backbone in red. The upper right-hand graph shows the transverse position of 3 different points on the cilium near the top, middle, and bottom of the image over time. The upwards trend is due to a sample drift of $\sim 0.1$ pixels/second, and the increased noise in the bottom trace (red) is from defocusing due to z-drift. One can see numerous discrete jumps in the traces caused by noise and drift combined with image pixelation. Correcting the transverse position for a constant drift of 0.1 pixels/second gives the bottom graph in Figure 4.6. The algorithm is able to track the cilium backbone with sub-pixel accuracy over at least 30 seconds, after which $z$-drift becomes a problem for the bottom section. During the first 30 seconds (750 frames), the standard deviations of the pixel position of the bottom, middle, and top points are $\sigma_{b}=0.17$ pixels, $\sigma_{m}=0.18$ pixels, and $\sigma_{t}=0.16$ pixels, respectively.

The second trial that we devised consisted of taking a still image of a cilium and adding to it a time course of camera noise. We did this in order to see how well our algorithm coped with the random noise and the non-homogeneous background present near the monolayer in our experiments. To do this we overlaid the still micrograph of the cilium in Fig. 4.1 with 3000 frames of image noise taken from the region immediately beside it and tracked its position. Figure 4.7 shows the results. The leftmost graph shows an overlay of all the tracked backbones. In the presence of noise, the algorithm does worse at localization, at some points deviating 2-3 pixels away from the centerline, or even more in the presence of background debris floating 

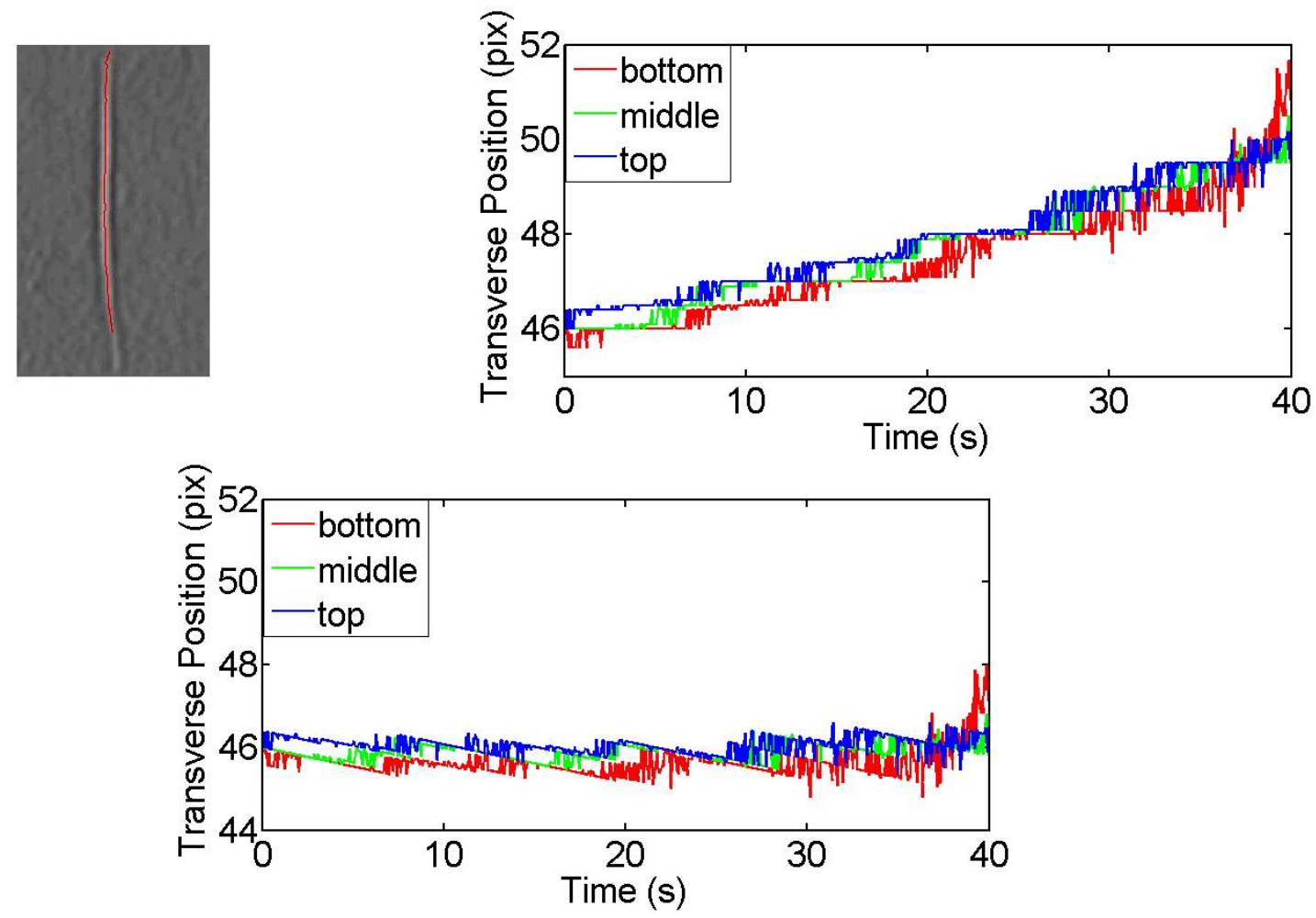

Figure 4.6: Performance of the tracking algorithm on a cilium affixed to a coverslip. The top left image is a micrograph of a cilium fixed to the coverslip, with a fitted backbone superimposed in red. The top right graph shows the algorithm tracking performance over time. There is a drift in the sample of $\sim 0.1$ pixels/second. At the end of the time series the red trace becomes noisy because of focal drift in the sample, and the lower portion of the image becomes defocused. The bottom graph shows the same time series except with a constant 0.1 pixels/second correction for drift. 

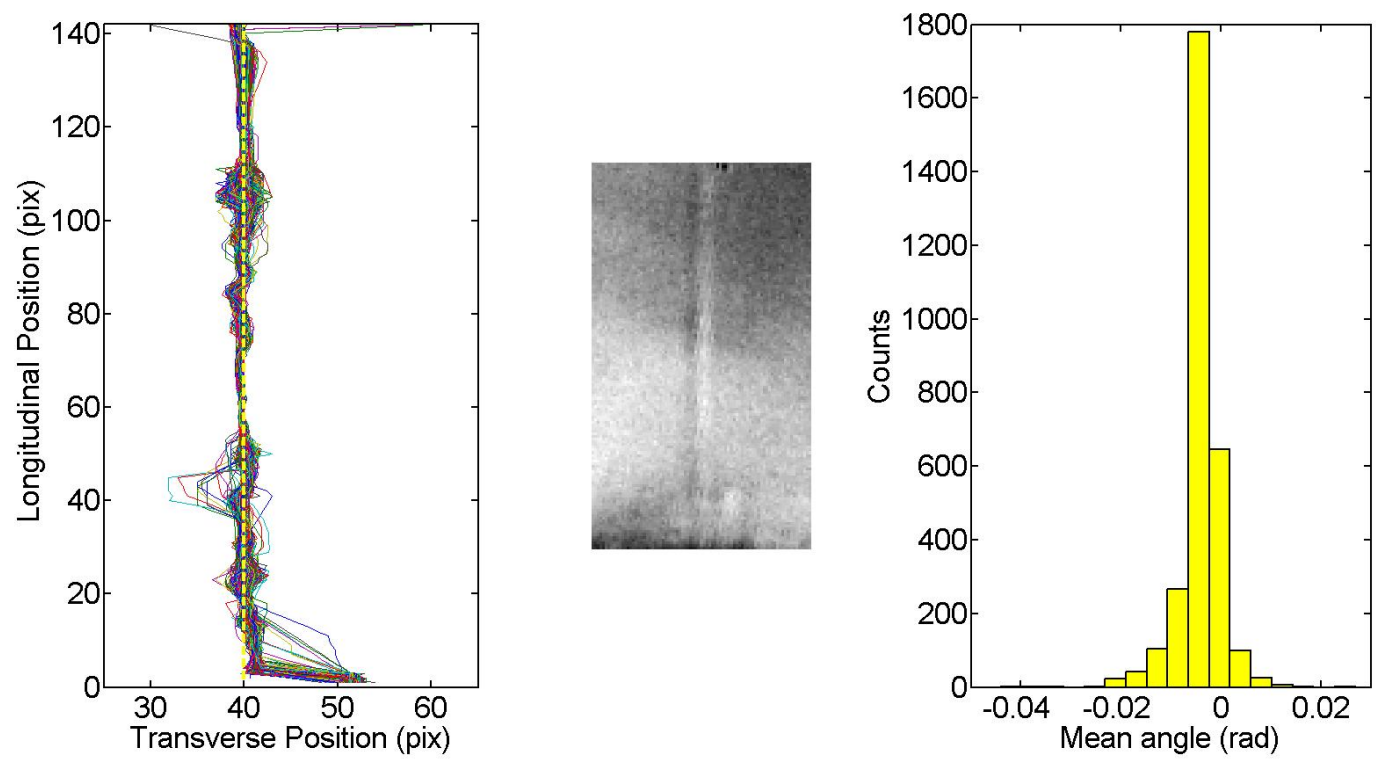

Figure 4.7: Tracking test of a stationary cilium with camera noise. A micrograph of the cilium is pictured in the middle. The leftmost picture shows an overlay of 3000 backbone tracked over two minutes. The yellow dotted line at transverse pixel value 40 is the centerline from which the mean angular deviation is calculated. The leftward bump at longitudinal pixel value 40 is due to debris diffusing through the frame. Below the longitudinal pixel value of 20 and at the very top the algorithm fails to accurately track the cilium. The rightmost graph shows a histogram of the cilium mean angle with respect to the centerline over the course of the movie, tracked between longitudinal pixels 25-100.

through the image (e.g. longitudinal pixel 40). Nevertheless, the algorithm does well on average, giving the backbone's mean position as transverse pixel $39.82 \pm 0.34$. The mean of the transverse position standard deviations of longitudinal pixels 25-100 is $\sigma_{B B}=0.34$ pixels, another indicator of good average localization accuracy. Next we calculated the mean angle the stationary backbone made with the centerline (at transverse pixel 40) in each frame to look at angular noise. The rightmost graph in Figure 4.7 shows a histogram of these values. The tracked cilium values have a peak slightly off-center from the centerline; this comes from the fact that there is a very slight curvature in the cilium and thus the tracked edge lies slightly to the left of the centerline for the lower half of the cilium. Since a greater portion of the lower half of the cilium is included in the analysis, this shifts the calculated mean angle off-center to negative values. Almost the entire distribution lies within a window of $\pm 1^{\circ}$, and the standard deviation of the mean angle distribution is even lower, at 
$\sigma=0.004 \mathrm{rad}\left(\sim 0.25^{\circ}\right)$. Though individual movies can have a worse noise floor, the noise level shown here is representative of the norm. Thus the perturbation to our tracking accuracy from background noise alone is less than a pixel on average, leading to less than a degree of deviation in the calculated mean angle.

\section{Base-shifting correction}

The main component of cilium motion that we see in our experiments is an angular rotation of the cilium about an equilibrium position. In general though, bending and translational motion ${ }^{2}$ of the cilium are also possible. In the cilium length ranges that we analyze, bending motion is negligible but translational motion does occur. Figure 4.8 shows an example of this translational motion. The four graphs depict groups of 500 superimposed backbones over different time intervals throughout the course of a movie. The base of the cilium is only centered on the centerline in the lower left-hand graph; in the other cases its center of mass moves several pixels away, both above and below the centerline. While such movement can be interesting in its own right, we would like to disentangle it from our calculations of the cilium's angular rotation, as it introduces systematic errors. Such errors show up as spurious curvature (e.g. a series of different angles that vary along the backbone length) which leads to a misestimate of the angular rotation. Figure 4.9 shows the different errors that base translations lead to. In the leftmost picture a pure rotation is shown, where all the angles the cilium makes with the centerline along its length are the same, i.e. $\theta_{1}=\theta_{2}$, where $\theta_{1}$ is the angle made by a point close to the cilium base and $\theta_{2}$ is the angle made by a point close to the cilium tip. The middle diagram depicts a base translation in addition to the original rotation in which the base crosses the centerline. In this case the angle between the cilium and the centerline is negative below a certain crossing point. Here $\theta_{1}<\theta_{2}$, the mean angle is underestimated, and the cilium appears erroneously to have convex-out curvature. Finally, the rightmost diagram shows a base translation away from the centerline in addition to the original rotation. Now the angles with respect to the centerline decrease along the cilium length, i.e. $\theta_{1}>\theta_{2}$. This leads to the opposite misestimate, in which the cilium appears to have convex-in curvature and the mean angle is overestimated. To correct for the base-shifting outlined above, we implemented the following steps in our algorithm, after the backbones have been determined and before we calculate the angular rotation of the cilium ${ }^{3}$ :

\footnotetext{
${ }^{2}$ Note that translational motion here includes various contributions: angular rotations where the hinge point is below the membrane, sample drift, and actual translations of the entire cilium from its initial position.

${ }^{3}$ We describe the steps for base-crossing shifts to the right and base shifting away from the centerline to the left, as depicted in Figure 4.8, but we also include the mirror-symmetric conditions for base-crossing shifts to the left and base shifting away from the centerline to the right in our
} 

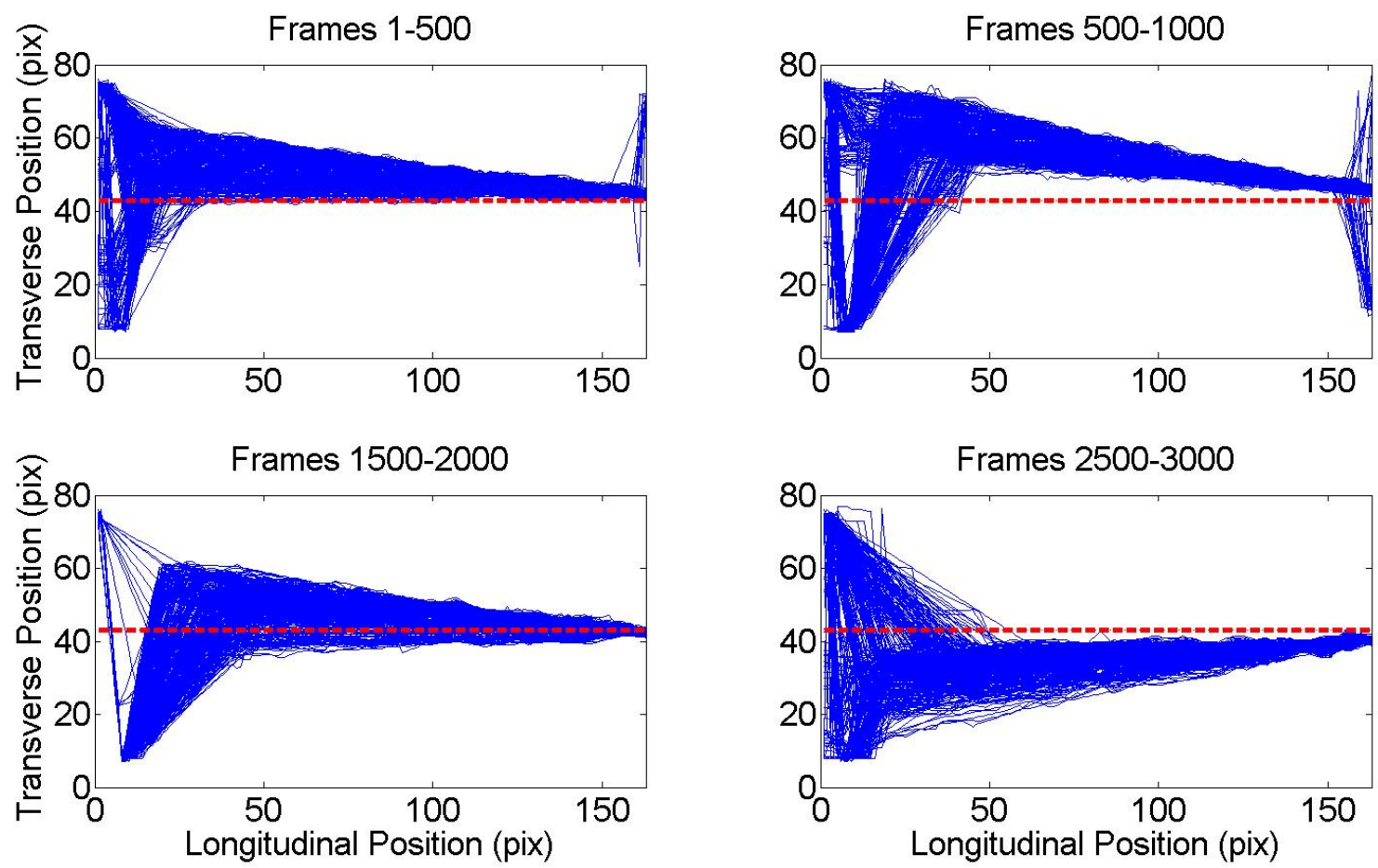

Figure 4.8: Shifting base problem. The four graphs each show an overlay of 500 tracked backbones (blue) for different time windows in a movie. The centerline is marked by the dotted red line and the longitudinal cilium axis lies along the x-axis, in contrast to the previous figures. In the lower left-hand figure, the cilium base lies on the centerline. In the other three figures, however, the cilium base position has shifted either above or below the centerline position, leading to errors in the calculation of the cilium's angular motion.

1. Get the values of the angles made by the three points closest to the cilium tip with the centerline in the analyzed region, $\left\{\theta_{\text {tip }}\right\}$, and the three angles closest to the base, $\left\{\theta_{\text {base }}\right\}$.

2. Check to see if the first case of base shifting is occurring (centerline crossing) by checking if $\forall \theta_{\text {tip }} \in\left\{\theta_{\text {tip }}\right\}, \theta_{\text {base }} \in\left\{\theta_{\text {base }}\right\}: \theta_{\text {tip }}>0 \& \theta_{\text {base }}<0$.

3. If condition 2 is fulfilled, we check to see if there is a unique centerline crossover point where the angles made by the points along the cilium with the centerline change from negative to positive (see Figure 4.9). This is to avoid shifting the backbone erroneously when it is close to zero angular displacement, as noise can cause the backbone to randomly cross back and forth over the centerline.

algorithm. 

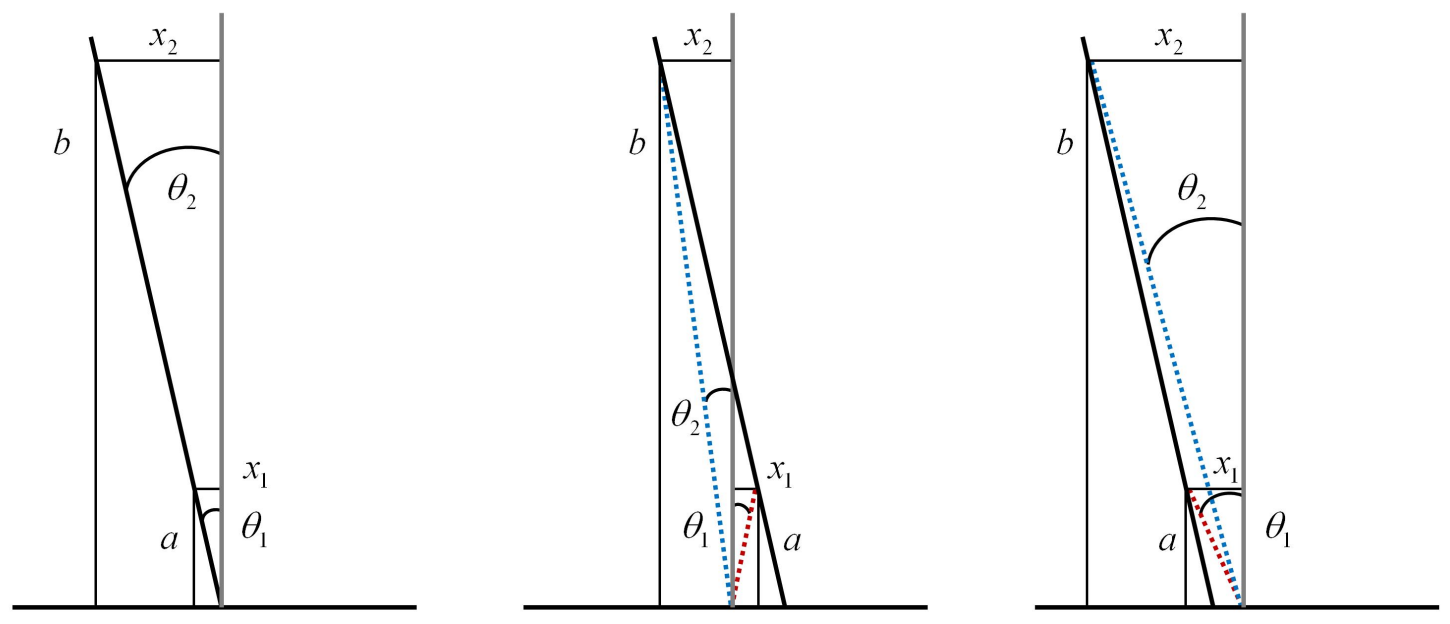

Figure 4.9: Schematic of angles involved in the base shifting correction. The light gray line is the centerline, the dotted red line represents the angle calculated for $\theta_{1}$, and the dotted blue line represents the angle calculated for $\theta_{2}$. The variables $a$ and $b$ are heights and $x_{1}$ and $x_{2}$ are horizontal displacements. On the left is a pure rotation of the cilium away from the centerline where $\theta_{1}=\theta_{2}=\arctan \left(x_{1} / a\right)=$ $\arctan \left(x_{2} / b\right)$. In the middle we have the same rotation pictured on the left but with the base shifted through the centerline. In this case $x_{1}$ and $\theta_{1}$ become negative and the angle made with the centerline is underestimated. In the right-hand drawing, a base shift away from the centerline is shown. Again when the two angles are calculated $\theta_{1}>\theta_{2}$, and the angle made with the centerline is overestimated.

4. If condition 3 is fulfilled, we calculate the crossover height, $a^{\prime}$, of the crossover point.

5. We then shift every point on the backbone by a distance $x^{\prime}=a^{\prime} \tan \theta_{1}$, where here $\theta_{1}$ indicates the angle made by the lowest tracked point on the backbone with the centerline. This brings the cilium base back to the centerline, negating the shift.

6. If $\overline{\left\{\theta_{\text {tip }}\right\}}<\overline{\left\{\theta_{\text {base }}\right\}}$, we conclude there is base shifting away from the centerline, where $\overline{\{\theta\}}$ designates the mean of the angles in the set $\{\theta\}$. To correct for this, at a height $a$ corresponding to the lowest tracked point on the backbone, we calculate $x=a \tan \theta_{1}$ and $y=a \tan \theta_{2}$, where $\theta_{1}$ and $\theta_{2}$ are the angles made by the lowest tracked point and the highest tracked point on the backbone with the centerline, respectively.

7. We then shift every point on the backbone by a distance $y-x$, bringing the cilium base back to the centerline position. 


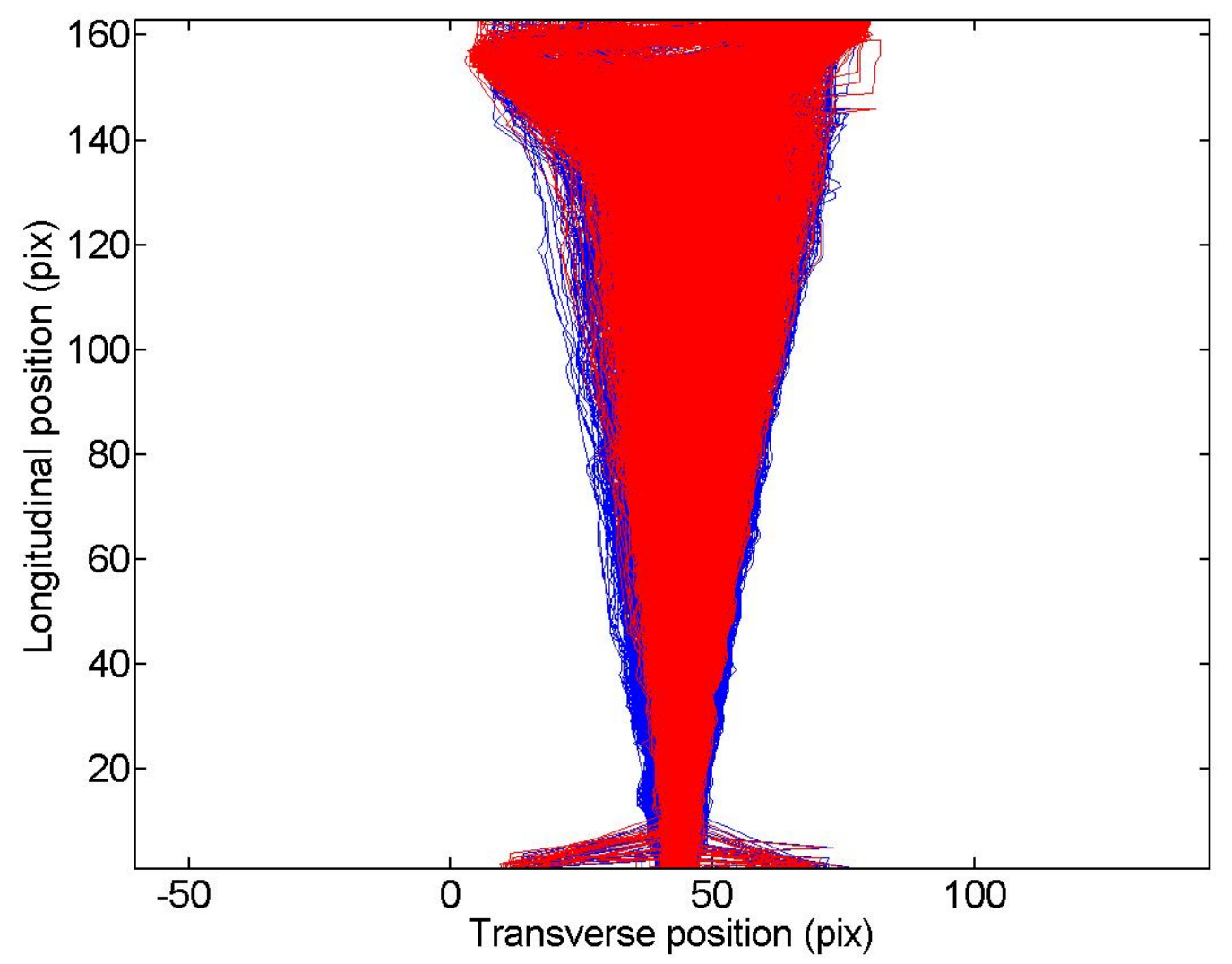

Figure 4.10: Graph of overlaid cilium backbones with base shift correction. The uncorrected backbone overlay is plotted in blue while the base shift corrected backbone overlay is plotted in red.

The effect of this base shifting correction is pictured in Figure 4.10, which shows the superimposition of backbones from the entire movie pictured in Figure 4.8. Here an overlay of 3000 backbones uncorrected for base shifting are pictured in blue while the base shift corrected backbones are drawn in red. While the correction noticeably "tightens" the backbone distribution, it is a relatively subtle effect. This is mostly likely due to the fact that translations occur over a relatively limited extent. In addition, the algorithm misses some translations, such as cases where base shifting crosses the centerline and the crossing point is at or below the lowest point analyzed on the cilium. All in all, while we include this correction in our analysis its impact is relatively minor in most cases . 

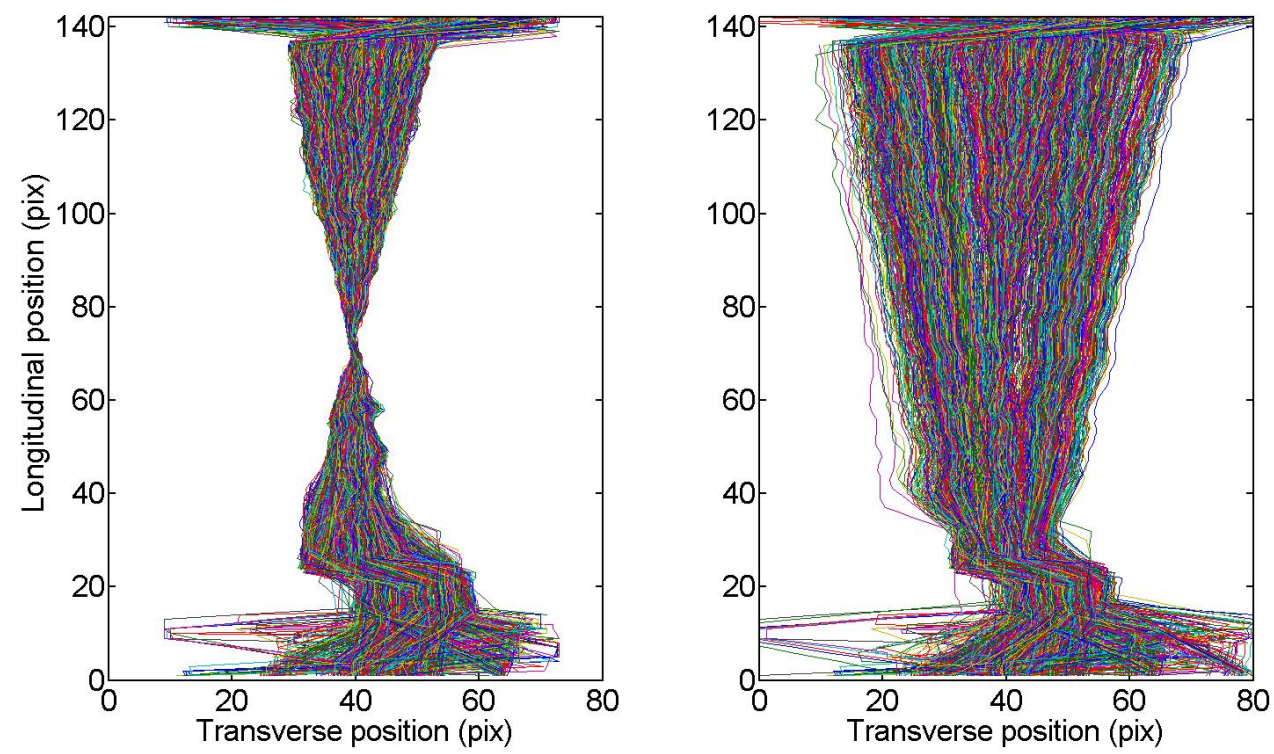

Figure 4.11: Rotation test of cilium tracking. A stationary cilium image (Fig. 4.1) was rotated in $1^{\circ}$ increments between $-10^{\circ}$ and $10^{\circ}$ about the image midpoint. On the left is an overlay of the 3000 tracked backbones. The tracking algorithm loses the cilium at the base because of an edge object parallel to the cilium (see the left picture in Fig. 4.5) which is co-rotated. The right-hand graph shows the cilium backbones corrected for base shifting. The algorithm is successful in deconvoluting the rotational motion from the translational motion but can't correct for the excursions at the base where the algorithm loses the cilium.

\section{Rotation test}

The main element of motion that we see from the cilium is a series of angular excursions about an equilibrium position. To test how well the algorithm can resolve angular rotations, we devised the following test: We took the movie used for the noise test above, in which a still cilium was overlaid with nearby background noise, and rotated the frame about its center. We executed the rotation in the pattern $0^{\circ}$ to $10^{\circ}$ to $0^{\circ}$ to $-10^{\circ}$ to $0^{\circ}$, in $1^{\circ}$ increments with the entire pattern repeated 75 times. A superposition of tracked backbones from this test can be seen in the left-hand graph in Figure 4.11. In the right-hand graph we can see the effect of the base shift correction discussed in the previous section; the base shifting is successful in deconvoluting the apparent base shifting (across the centerline) caused by the rotation about the center point. Unfortunately the base shifting correction fails for small base shifts due to the large kink in the backbone below longitudinal pixel value 40. This kink leads to multiple zero crossings at small angles and consequently the 

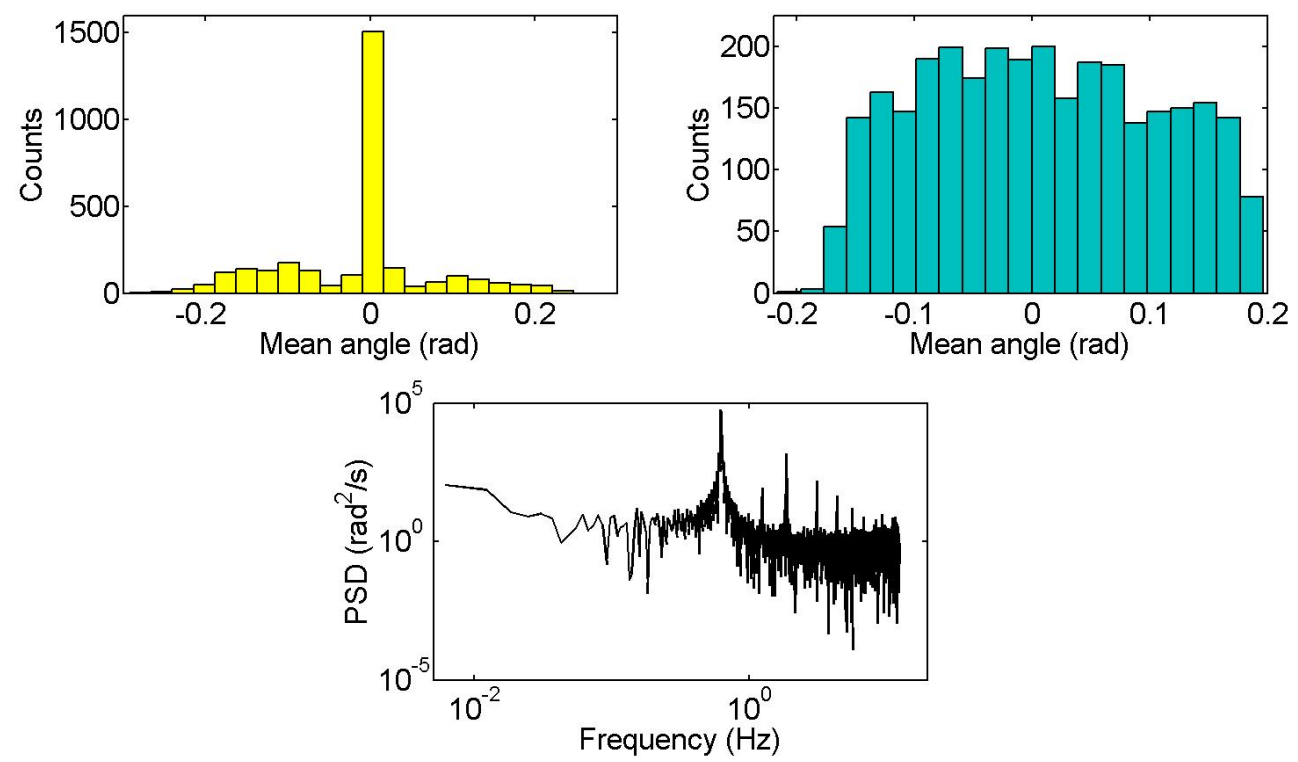

Figure 4.12: Graphs of the algorithm's performance on the rotation test. The upper left graph shows a histogram of calculated mean angle values with the base shift correction between longitudinal pixel values of 32-132. The algorithm grossly overestimates the number of values in the center bin. The upper right graph shows the algorithm's performance when just the sections above the midpoint are analyzed (pixels 82-132). Here the algorithm perfoms much better, coming closer to the real values (150 counts in all the bins except the two outermost, which each have 75 , between -0.17 and 0.17 radians). The bottom graph shows the power spectral density of the angular displacements. The peak corresponds to the 40 frame period of the rotational motion (the graph assumes a $25 \mathrm{~Hz}$ frame rate).

algorithm doesn't implement the base-shifting correction for these angles. This leads to a severe overestimation of the number of events in the 0 bin (see upper left graph in Figure 4.12) since the negative values of the uncorrected angles below the hinge point are added to the positive values above it and cancel out. We can do significantly better by looking at the rotation of the uncorrected series of backbones above the hinge point (longitudinal pixel value 71 ). If we calculate the mean angle above this point we get the histogram shown on the upper right of Figure 4.12. Here the values in each bin scatter around the actual value of 150 (except in the two outermost bins where the real value is 75). Analyzing the power spectrum of the time series picks out the periodic nature of the signal, as shown in the lowermost graph of Figure 4.12. A peak is readily apparent in the power spectrum at $0.625 \mathrm{~Hz}$, which corresponds to the 40 frame repetition cycle of the rotational motion, assuming a acquisition rate 
of $25 \mathrm{~Hz}$. To get an estimate for the angular resolution of our tracking, we compare the number of incorrect counts with the total number of counts from the right-hand histogram in Fig. 4.12. This gives a failure rate of $151 / 3000 \approx 5 \%$. Conversely, the algorithm tracks rotations in $1^{\circ}$ increments with a success rate of $95 \%$.

\section{D Tracking}

In the Results chapter we also present collaborative data taken by Carolyn Ott in which we reconstruct confocal-stack time series data of fluorescent cilia taken in a flat geometry. The tracking and reconstruction performed on this data is much more straightforward than the algorithm just described. Fluorescent cilia seen from above appear as point sources and there are myriad open source spot-tracking programs available. We use an ImageJ plug-in called SpotTracker written by Daniel Sage, documentation of which is available at http://bigwww.epfl.ch/sage/soft/spottracker/ and in Ref. [71]. SpotTracker tracks point-like fluorescent particles by searching for a maximal intensity point (or user defined node) and from that point it solves the global optimum problem for the time trajectory of the particle displacements. Solving this optimization problem is made feasible by introducing user defined constraints on the maximum possible frame-to-frame distance traveled by the particle, which are incorporated into a cost function. The optimization problem is solved using the dynamic programming approach in which the global optimization is broken down into the problem of step-wise optimization, from which the globally optimal trajectory is recovered recursively. To reconstruct a 3D time course of a cilium from confocal data we perform the following steps:

1. Isolate the cilium of interest by cropping the movie (SpotTracker can only track one object of interest at a time).

2. Pre-filter the confocal $x y z t$ stack with a Gaussian blur filter to reduce noise.

3. Run SpotTracker on the whole $x y z t$ stack, which gives $x y$ coordinates for the cilium in each slice (see Figure 4.13, left-hand picture).

4. Load the $x y$ coordinates of each slice into Matlab and separate them into a series of 3D cilium backbones (see Figure 4.13, right-hand picture) over time.

It should be remarked that running SpotTracker over the entire xyzt stack adds a somewhat funny constraint to the optimization problem, that of connecting the tip of the cilium in one time step to the base of the cilium in the next. Strictly speaking it would be more correct to track the time trajectory of each individual $z$ slice separately. In practice however, due to the decent SNR of the confocal stacks, it makes little difference in the coordinate list whether or not we run SpotTracker 

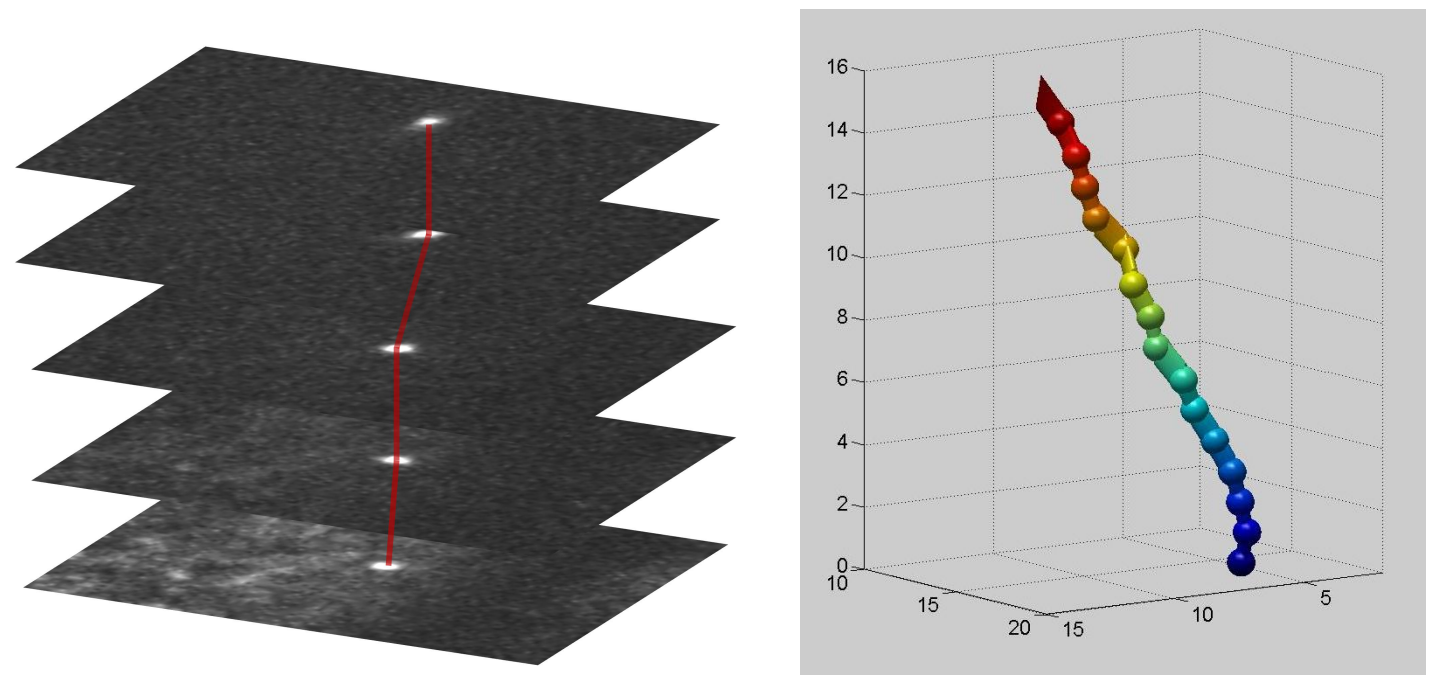

Figure 4.13: Schematic of the 3D reconstruction of confocal-stack time series data. The left-hand picture depicts a confocal stack where the red line joining the different frames represents the optimal $(z)$ trajectory between the slices found by SpotTracker. The right-hand picture shows a 3D cilium backbone reconstructed in MatLab using the coordinate list returned by SpotTracker. The axes scales in the right-hand picture are all in micrometers.

on the whole $x y z t$ stack or break it up into separate $x y t$ stacks for each $z$. Since it is faster and more convenient to analyze the whole stack at once, we stick to the steps prescribed above when possible.

\section{Discussion}

In this chapter we've laid out the details of the algorithm we wrote to perform cilia tracking in DIC movies. There are many, many different ways to solve the problems of feature extraction and trajectory reconstruction, some much more sophisticated than the method presented here. The most obvious improvement to our technique would be to implement a strategy whereby we find an optimal trajectory for each point on the cilium from frame to frame, such as the sum-of-square differences (SSD) technique implemented in Ref. [68]. While implementing such a technique could improve our backbone tracking, it is computationally costly. Since we are mostly interested in the large scale motion of the cilium, which our algorithm accurately tracks, we have to date not implemented such improvements but they are always possible in the future. Other provisions in the algorithm, such as the constraints of the filter width on movement and the number of frames used for outlier rejection, 
are somewhat ad hoc and don't have strong a priori arguments for their specific values, which have been determined by user observation of cilium dynamics and trialand-error. Nevertheless, "the proof is in the pudding" and our approach functions robustly for the type of data presented in this work. In conclusion, our algorithm is able to track cilium position under ideal circumstance with sub-pixel accuracy and can resolve angular displacements of $1^{\circ}$ with a $95 \%$ accuracy even in the less-thanideal environment present in our movies. 


\section{Chapter 5}

\section{Experimental methods}

In this chapter we describe methodological details of the experiments described in the Results chapter. Step-by-step protocols for the various methods discussed here are included in Appendix A. We discuss cell cultivation on flexible membranes, sample chamber construction, bead coating, micropipette pulling, and the methodology used in the electrophysiology/calcium imaging/ confocal imaging experiments.

\section{Cell cultivation on flexible membranes}

Primary cilia in kidney epithelial cells grow from the apical side of confluent cell monolayers. The left-hand side of Figure 5.1 shows cells near-confluence, some of which likely have primary cilia. However, since primary cilia have a diameter at or below the diffraction limit and typically grow almost parallel to the optical axis, they are invisible when viewed from above. To overcome this problem we adopted a technique from Reference [72], in which cells were grown on a flexible substrate and then bent over so that they can be imaged from the side. When one images the edge where the cell sheet is bent, the primary cilia extend out perpendicular to the optical axis and are readily visualized with phase-contrast techniques. The righthand plate in Fig. 5.1 shows a Whatman Nuclepore polycarbonate membrane (PCM) which we use as a flexible substrate on which to grow our cell line. We use $25 \mathrm{~mm}$ circular membranes with a $400 \mathrm{~nm}$ pore size (Whatman PLC., 110607). The PCM membranes are porous which has the added advantage of allowing fluid transport from the apical through the basal side of the cell. When this transport is restricted, as with cells grown on glass, the cell sheet starts to form blisters of trapped water after being confluent for a day or more. In order to cultivate MDCK-II cells (the only cells used in this study) on PCMs, we first sterilized them with UV light then soak them in poly-L-Lysine (PLL) solution for 10-30 minutes. The PLL solution facilitates cell attachment, though MDCK cells will grow on the membranes without it, albeit more slowly. Once this was done we subcultivated the cells as outlined in 

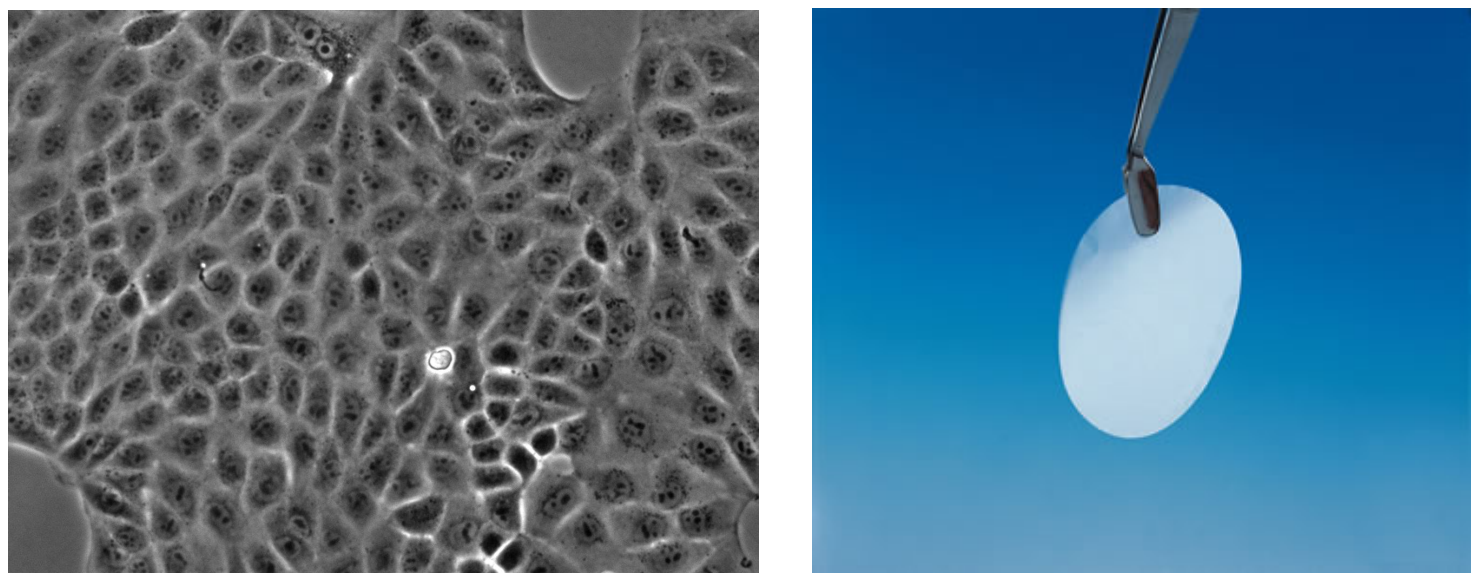

Figure 5.1: Phase-contrast picture of MDCK-II cell sheet near confluence (left) and a Whatman Nuclepore polycarbonate membrane (right), on which we culture the cells for our experiments. The cell sheet pictured is slightly beyond the level of confluence which we usually subcultivate at. The right-hand picture is taken from Whatman's website http://www.whatman.com/NucleporeTrackEtchedMembranes.aspx.

Appendix A. We then incubated the cells at $37^{\circ} \mathrm{C}$ in a $5 \% \mathrm{CO}_{2}$ environment for 6-7 days. The cells formed a confluent sheet on the 5th or 6th day and we performed our experiments after they'd been confluent for 1-2 days. All cells used for experiments had a passage number of 35-55.

\section{Sample chamber construction}

To mount our samples we constructed three different types of homemade sample chambers. The first two types consisted of a microscope slide and coverslip joined together by double-stick tape and were used with the dual-optical trap/DIC microscope described in Chapter 3. We term these chambers "closed chambers" and they are described in the next section. The third type of chamber, used for sharp-electrode recording experiments, was used with the optical-trap/patch-clamp/fluorescence microscope setup described in Chapter 2. This chamber type we term an "open chamber" and its construction is detailed in the eponymous subsection.

\section{Closed Chambers}

As described in the previous section, we cultured cell monolayers on flexible membranes so that they could be bent over with the cilia extending perpendicular to the optical axis. A diagram of this edge-viewing technique is shown on the left-hand side of Figure 5.2. We image the sheet at or close to the edge of the bent sheet, where the 

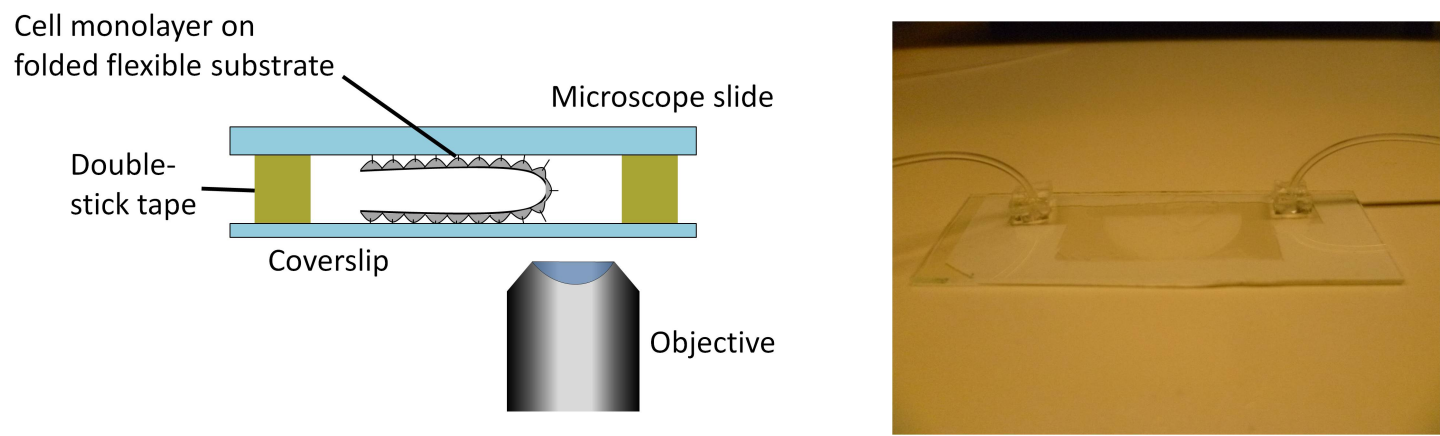

Figure 5.2: Diagram of the edge-viewing technique (left) and a picture of a homemade flow chamber with folded PCM mounted inside (right). The PCM in the right-hand picture appears as a semi-circular white object in the middle of the flow chamber. Closed and sealed chambers, as pictured in the diagram, were used for cilium fluctuation analysis and are identical to the flow chambers pictured above except that they lack inlets.

cilia are most clearly visible. Most of the images presented in this work were taken using this imaging geometry (see Chapters $2 \& 6$ for examples). Two different types of chambers for the optical trapping and fluctuation analysis experiments were constructed. The first type of chamber, used in the optical trapping experiments with beads, is pictured on the right-hand side of Fig. 5.2. To construct this type of chamber we sandblasted two $2 \mathrm{~mm}$ diameter holes in the microscope slide approximately $45 \mathrm{~mm}$ apart. We then cured polydimethylsiloxane (PDMS) in a large Petri dish and cut out squares (approx. $7 \mathrm{~mm} \times 7 \mathrm{~mm}, 5 \mathrm{~mm}$ thick) to serve as inlets. We made holes in the squares using a $2 \mathrm{~mm}$ punch and mounted the PDMS inlets on the sandblasted microscope slides so that the holes lined up with each other. The PDMS and glass were bonded together using super glue (UHU GmbH, cyanoacrylate adhesive). The bond made was moderately durable; it would remain water-tight for medium flows, but higher pressures could cause leakage. Thin double-stick tape (Tesa SE, 4960, $50 \mathrm{~mm}$ wide, $\sim 80 \mu \mathrm{m}$ thick) was taped across the microscope slide and cut to accommodate the inlets and the folded sheet, as pictured. When the cells formed a confluent sheet on the PCM the membrane was taken out of the 6-well, folded over, and mounted so that the folded edge lined up with the inlets. Then $\sim 50 \mu \mathrm{L}$ of bead-containing solution $(1.5 \mu \mathrm{m}$, diluted 1:5000 in culture medium from $50 \mathrm{mg} / \mathrm{mL}$ bead stock solution) was added and the chamber was sealed, with a long coverslip $(24 \times 60 \mathrm{~mm})$ making up the other side. To allow flow into/out of the inlets, we inserted silicone tubing (Helix Medical, mono lumen tubing, $0.76 \mathrm{~mm}$ ID x $1.67 \mathrm{~mm}$ OD x $0.45 \mathrm{~mm}$ wall thickness), attaching one length of tubing to a syringe pump (Harvard Apparatus, Harvard Apparatus Standard 22, 55-2226) for inflow. The end of the other length of tubing was placed into a Petri dish to collect the outflow. Flow 
was generated using $2 \mathrm{~mL}$ syringes (Becton Dickinson GmbH, BD Discardit II) with 21 gauge needles (Terumo Co., Neolus green needles, $0.8 \times 37.6 \mathrm{~mm}$ ) at flow rates of $50-100 \mu \mathrm{m} / \mathrm{min}$. Before the experiments were performed flow was initiated and left on for 2-5 minutes to wash out cellular debris. The solution added was either the standard culture medium described in Appendix A (MEM Earle's with L-glutamine and FBS) or $\mathrm{CO}_{2}$-independent medium (Life Technologies, Gibco, 18045-054, with $2 \mathrm{mM}$ L-glutamine, $1 \%$ penicillin-streptomycin, and 10\% fetal bovine serum). We pumped solution into the chamber rather than sucking it out of a reservoir through the chamber to avoid trapping air bubbles inside. The flow rates used didn't wash out the beads included when the chamber was assembled, which were large enough to settle to the bottom of the chamber before flow was initiated. The coverslip was coated with dimethyldichlorosilane (DDS) to prevent bead adhesion.

The second type of closed chamber, used for the fluctuation analysis and bend \& relax experiments, is a simpler version of the chamber just described. Instead of sandblasting holes and mounting inlets we used a standard microscope slide and followed the mounting steps detailed above, creating an entirely sealed chamber. We did this in order to eliminate flows in the sample which could influence the cilium fluctuations. Upon mounting the PCM, $\sim 50 \mu \mathrm{L}$ of standard or $\mathrm{CO}_{2}$-independent medium (with no beads included) was added to the chamber; otherwise the steps were as detailed above.

\section{Open Chambers}

The third type of chamber that we constructed was open from above to allow access for a water-dipping objective and micropipette. A diagram of a sharp-electrode recording experiment using this chamber is shown in Figure 5.3, as well as a photo of the chamber alone. The upper part of the chamber was machined out of polyvinyl chloride (PVC) and bonded to a microscope slide using two-component glue (Huntsman Co., Araldite 5 minute AB epoxy). Two $3 \mathrm{~mm}$ diameter rare earth magnets were glued into recesses in the PVC for sample mounting. MDCK cells were cultured on a flexible sheet and bent over for edge-viewing as described above. The bent over cell sheet was held in place by two $3 \mathrm{~mm}$ diameter rare earth magnets which attached to the complementary magnets mounted in the chamber. The sample chamber was then filled with $\sim 1 \mathrm{~mL}$ of medium (culture or $\mathrm{CO}_{2}$-independent). This overfilled the chamber so that there was a meniscus above the cell sheet, into which the water-dipping objective was lowered. The micropipette was then positioned at a very shallow angle, close to horizontal, so that it came in almost normal to the bent edge. A shallow angle was necessary to reach the membrane edge and allow the pipette to fit underneath the objective. After experiments the chamber was washed with $70 \%$ ethanol and reused. 

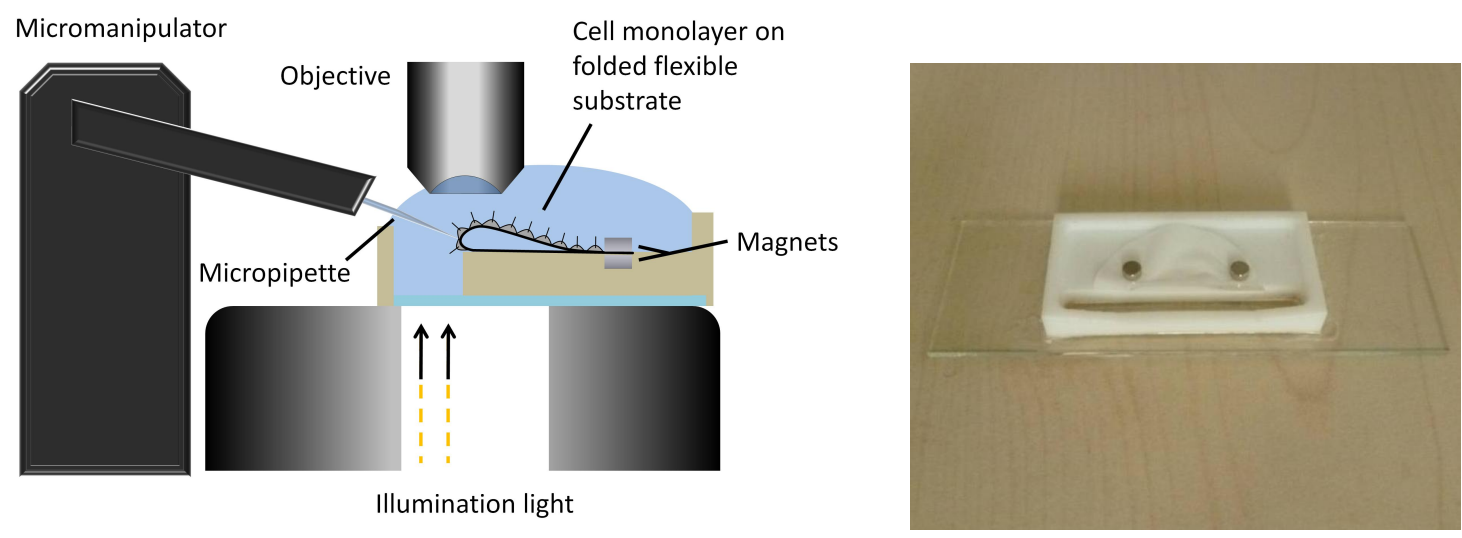

Figure 5.3: Diagram of an open chamber sharp-electrode experiment (left) and a picture of an open chamber with a folded PCM (right). The PCM in the right-hand picture appears as a semi-circular, slightly wrinkled white object on top of the white chamber, held in place by two magnets.

\section{Bead antibody coating}

To attach beads to cilia for the relevant optical trapping experiments, we coated $1.5 \mu \mathrm{m}$ carboxylated glass beads with antibodies against polycystin-1 (PC1), a transmembrane protein present in the primary cilium of kidney epithelial cells. PC1 has a large extracellular N-domain with a leucine-rich repeat flank region (24-180aa) [73] and the antibody used targets this region (Abcam PLC, Anti-Polycystin 1 antibody, [7e12], ab74115). To attach beads to cilia we brought the bead into contact with the cilium for 1-2 minutes using an optical trap. We then moved the trap back and forth to make sure that the cilium and bead were firmly attached. A protocol for coating beads with antibodies can be found in Appendix A.

\section{Micropipette pulling \& electrophysiology}

Micropipettes for sharp electrode recording were pulled on a horizontal pipette puller (Sutter Instument Co.,model P-97) from borosilicate glass (Science Products GmbH, GB100F-8P,0.58 ID x 1.00 OD x $80 \mathrm{~mm}$, with filament, fire-polished ends). The following pulling protocol was used: Heat=Ramp, Pull=80, Velocity $=70$, Delay $=80$, Pressure $=200$. This yielded pipettes with a short shank and a resistance of $10-20 \mathrm{M} \Omega \mathrm{s}$ (in $3 \mathrm{M} \mathrm{KCl}$ ), an acceptable range for sharp electrode recording. The intracellular solution used for sharp electrode recording was $3 \mathrm{M} \mathrm{KCl}$ while the extracellular solution was the same as for the patch clamp experiments (see below).

Patch clamp pipettes were pulled on a vertical pipette puller (List Medical, L/M- 
3P-A) using a two-pull protocol (15.3 A current for the first pull followed by $11 \mathrm{~A}$ current for the second pull). The glass used was Corning \#0010, a high-lead content glass (World Precision Instruments, PG10165-4, $1.65 \mathrm{~mm}$ OD, $1.1 \mathrm{~mm}$ ID) and the pulling protocol resulted in patch pipettes with a resistance of $2-5 \mathrm{M} \Omega$.

Electrophysiological recordings were performed in the whole-cell patch clamp mode [20] using an EPC10 patch clamp amplifier (HEKA Elektronik, Lambrecht, Germany). Cells were visualized using an inverted microscope (Zeiss Axiovert 135). Pulse protocol generation and data acquisition were controlled with Patchmaster

software (HEKA Elektronik). Currents were low-pass filtered at $10 \mathrm{kHz}$ and sampled at $50 \mathrm{kHz}$. Data was analyzed with Matlab. The extracellular solution consisted of (in $\mathrm{mM}$ ) $134 \mathrm{NaCl}, 4 \mathrm{KCl}, 2 \mathrm{CaCl}_{2}, 1 \mathrm{MgCl}_{2}, 10 \mathrm{HEPES}$, and 20 glucose, adjusted to $\mathrm{pH} 7.4$ with $\mathrm{NaOH}$. The intracellular solution consisted of (in mM) $136 \mathrm{~K}-\mathrm{Glu}, 5$ $\mathrm{NaCl}, 0.1 \mathrm{EGTA}, 10 \mathrm{KCl}, 10 \mathrm{HEPES}, 1 \mathrm{MgATP}, 0.3 \mathrm{NaGTP}$, and 5 Phosphocreatine, adjusted to $\mathrm{pH} 7.2$ with $\mathrm{KOH}$. All experiments were performed at room temperature.

\section{Calcium imaging}

All calcium imaging experiments were performed on an inverted microscope (Nikon, te200) using a monochromator for illumination (TILL Photonics, Polychrome V). Emitted fluorescence was coupled out of the beam path via a $505 \mathrm{~nm}$ long-pass dichroic mirror (Chroma, T505lp) and was filtered through a $510 \mathrm{~nm}$ long-pass filter (Chroma, ET510lp) before detection. Fluorescence signal was detected with an EMCCD camera (Andor Technology, iXon ultra) at a gain of 300. Flow was regulated with a Warner instruments six-channel perfusion valve control system with external trigger control (Measurement Computing, PCIM-DDA06/16). Image acquisition was performed using Andor iQ 2.6 imaging software (Andor Technology) Data analysis was done in Matlab.

MDCK cells transfected with a Smoothened-YFP construct, which localizes to the cilium (courtesy Carolyn Ott), were cultured to confluence on $18 \mathrm{~mm}$ glass coverslips $\left(0.17 \mathrm{~mm}\right.$ thick) and then incubated for 30 minutes at $37^{\circ} \mathrm{C}$ with Fura 2AM dye $(2.5 \mu \mathrm{M})$ in Ringer solution consisting of (in $\mathrm{mM}$ ) $160 \mathrm{NaCl}, 2.5 \mathrm{KCl}$, $2 \mathrm{CaCl}_{2}, 1 \mathrm{MgCl}_{2}, 10 \mathrm{HEPES}$, and 8 glucose. After dye loading, the cells were washed to get rid of excess dye and an extracellular solution of Ringer's solution with $2 \mathrm{mM}$ calcium was added. The coverslip was mounted in a custom imaging chamber and left undisturbed on the microscope for $\sim 10$ minutes before the start of the experiment. Images were acquired at $2 \mathrm{~Hz}$ with an excitation sequence of: $340 \mathrm{~nm}$ for $100 \mathrm{~ms}, 400 \mathrm{~nm}$ for $100 \mathrm{~ms}, 488 \mathrm{~nm}$ for $100 \mathrm{~ms}$. Emission light above $510 \mathrm{~nm}$ was collected during each excitation. The protocol followed in each experiment was as follows: The sample was imaged, first for 20 seconds without flow, then for 120 seconds with flow on, then for 120 seconds with flow off. All experiments were 
performed at room temperature.

\section{Confocal data}

All confocal data collection was performed by Carolyn Ott (National Institutes of Health, Cell Metabolism and Biology Branch, Lippincott-Schwartz lab). She generated stable cell lines by transfecting MDCK II cells with emerald-MCHR (created by Mike Davidson) and mRFP PACT (created by Alex Ritter) constructs using FuGene transfection reagent (Promega). MCHR1 (melanin-concentrating hormone receptor 1 ) is a membrane protein which localizes to the primary cilium while the PACT (pericentrin-AKAP450 centrosomal targeting) domain targets the centrosome in mammalian cells. Two days after transfection the cells were split and G418 was added for selection $(960 \mu \mathrm{m} / \mathrm{mL})$. Fluorescent protein expressing cells were sorted using FACS to create a stably transfected cell line.

MDCK II cells were trypsinized and plated at $1.17 \times 10^{4}$ cells $/ \mathrm{cm}^{2}$ in LabTek cover glass chambers (Nunc 155383). After the first two days, cell media was exchanged daily. The culture medium used was Minimum Essential Medium (Corning, 10-010-CV) with $3 \mathrm{mM}$ L-glutamine (Corning, 25-005-CI) and $10 \%$ fetal bovine serum (Corning, 35-011-CV). The medium was supplemented with G418 $(960 \mu \mathrm{m} / \mathrm{mL})$ for selection. Cells were imaged using a spinning disk confocal (Marianas, Intelligent Imaging Innovations) with a $63 \mathrm{x}, 1.4 \mathrm{NA}$ oil-immersion objective at either $37^{\circ} \mathrm{C}$ or room temperature. Cilia were visualized at $z$ intervals of $1 \mu \mathrm{m}$ unless otherwise specified. The number of slices in a stack varied from 5-16, with a concomitant variation in acquisition speed. 


\section{Chapter 6}

\section{Results}

In this chapter we present the results of the various micromechanical and tracking experiments performed on the primary cilia of MDCK-II cells, as well as some preliminary results probing their mechanosensitive response. We first describe results obtained with the DIC/dual-optical trap setup described in Chapter 3 relating to cilium mechanics, cilium boundary conditions, and cilium fluctuation dynamics. In the subsequent sections we describe collaborative results, namely the analysis of 3D cilia tracking experiments performed by Carolyn Ott (NIH) and collaborative electrophysiology/calcium imaging experiments done with Andreas Neef (MPI-DS) and Andrew Woehler (MPI-BPC). A note to the reader on conventions: unless otherwise noted the error bars given are \pm one (sample) standard deviation. All experiments were performed at room temperature, unless otherwise noted.

\section{Cilium mechanics}

One of the main material parameters of interest for an isotropic, homogeneous rod is its bending stiffness (also referred to as flexural rigidity), EI. This parameter describes how a rod responds to externally applied load, and from it one can obtain the deformation profile of a rod in a given force field [74]. Since the mechanosensitive response of the primary cilium is thought to depend upon bending under shear flow $[11,14,75,76]$, the flexural rigidity is clearly a relevant parameter to measure if one wants to understand ciliary mechanosensation. Given that the bending stiffness is a factor which controls cilium curvature and force transmission from the cilium to the cell, it could affect various biophysical details such as mechanosensitive channel gating and channel location, about which there is some debate [77]. It can be shown that, for a cantilevered beam of length $L$ with one end fixed and a force $F$ applied

at the other, the deviation from the equilibrium position, $z(x)$, at a point $x$ is given by 


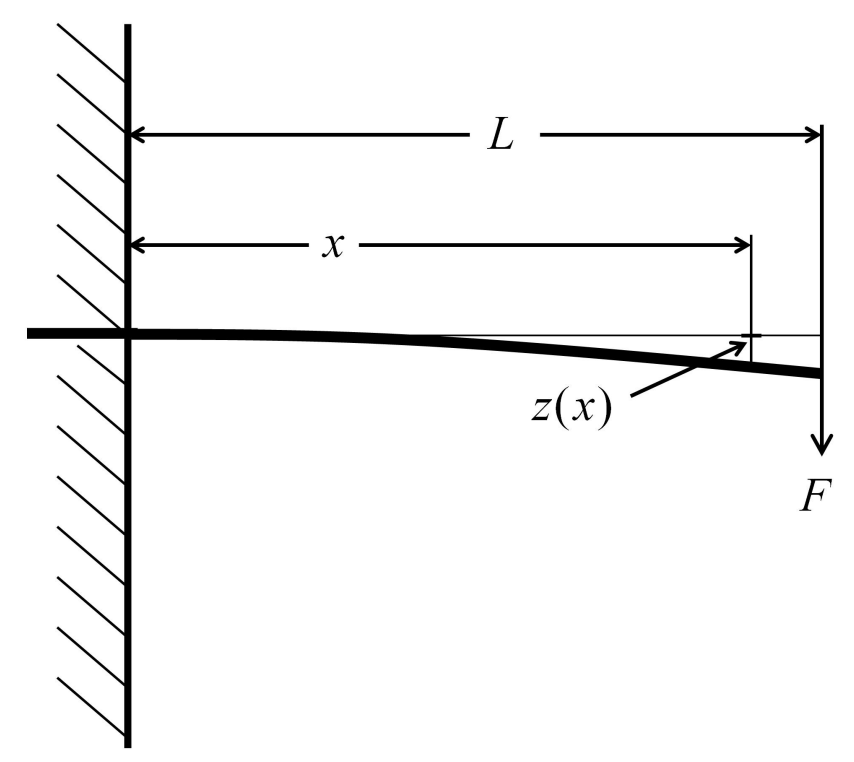

Figure 6.1: Diagram of clamped cantilevered beam. A force $F$ is applied at the free end, giving a transverse displacement $z(x)$ at a point $x$.

$$
z(x)=\frac{F}{E I}\left(\frac{L x^{2}}{2}-\frac{x^{3}}{6}\right)
$$

in the limit of small deflections [74]. Figure 6.1 shows a diagram of the relevant variables for the situation described. From Eqn. 6.1 it immediately follows that the flexural rigidity is given by

$$
E I=\frac{F}{z(L)} \frac{L^{3}}{3}
$$

where $z(L)$ is the displacement of the rod's end. In the following we determine the flexural rigidity using the above relations by measuring the displacement of the cilium, treated as a cantilevered beam, under a given load delivered via an attached bead held in an optical trap.

\section{Active bending experiments}

In order to deliver a controlled force to the cilium, we first attached a $1.5 \mu \mathrm{m}$ antibody-coated silica bead as described in the previous chapter, to make a construct like the one pictured in Figure 6.2. Briefly, we brought a trapped bead into contact with the cilium, usually for about a minute, until a stable bead-cilium connection was formed. We then performed our "active bending experiment": With the bead held in the optical trap, we drove the piezo stage sinusoidally in the plane approximately 

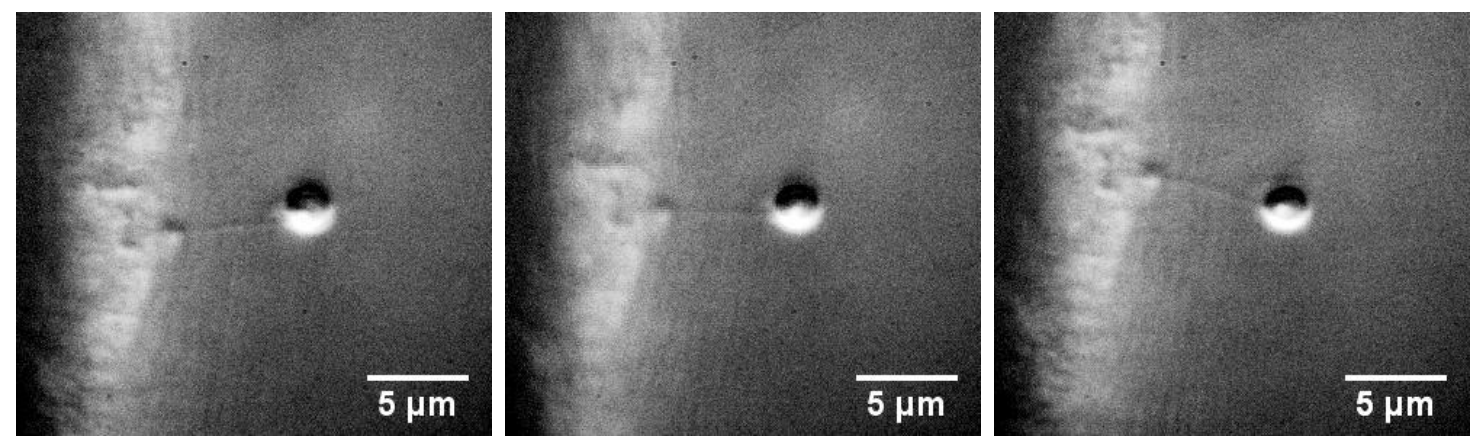

Figure 6.2: Series of stills from an active bending experiment. The bead is moved back and forth in the vertical plane, approximately parallel to the cell sheet, by moving the piezo stage with a sinusoidal driving signal.

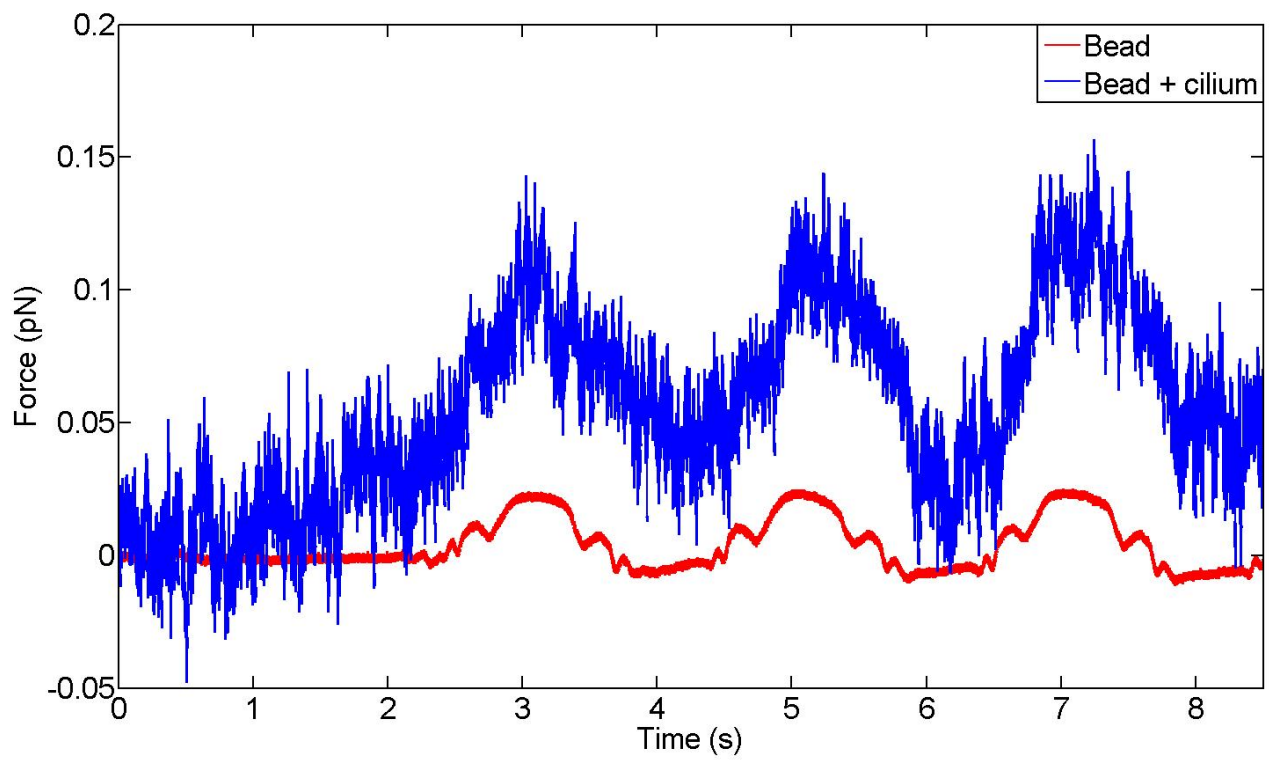

Figure 6.3: Force traces from an active bending experiment. The blue trace corresponds to the active bending experiment pictured in Fig. 6.2 while the red trace corresponds to a control measurement. The control measurement shows the signal generated from moving a bead back and forth near the sheet, approximately in the same region and at a distance roughly equal to the cilium length.

parallel to the cell sheet, (north-south in Fig. 6.2) with an amplitude of $2.5 \mu \mathrm{m}$. We concurrently measured the quadrant photodiode (QPD) signal and recorded a DIC movie of the experiment at $25 \mathrm{~Hz}$, in order to extract the force on the bead and its displacement. 


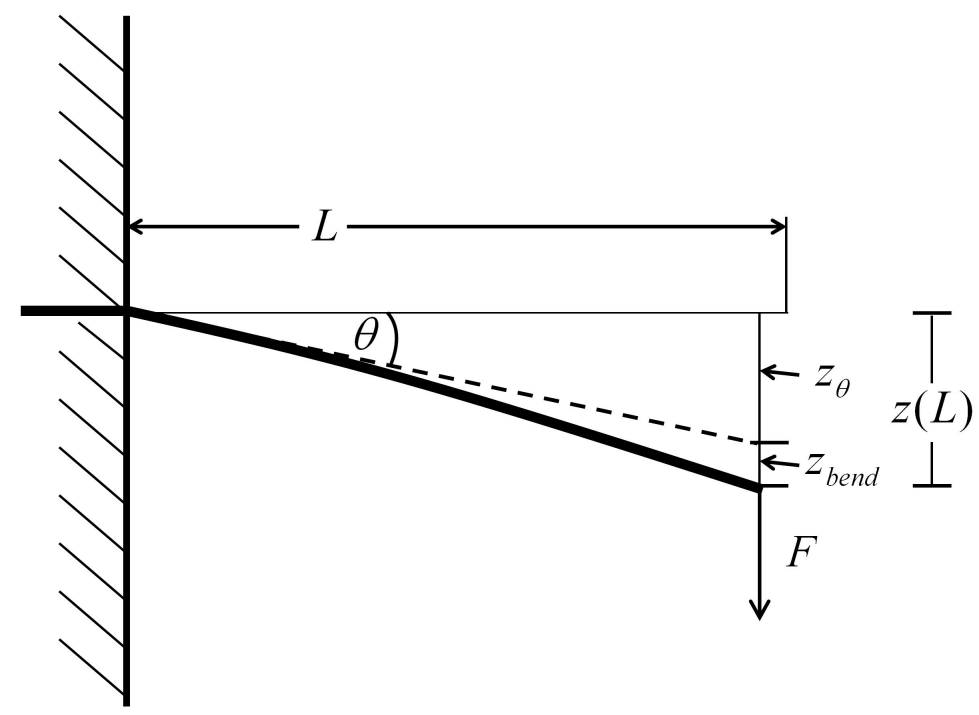

Figure 6.4: Diagram of a cantilevered beam with a limited hinge. A force $F$ is applied at the free end, both bending it and rotating it through an angle $\theta$. Here $z(L)$ is the total transverse displacement of the beam at its tip, $z_{\theta}$ is the displacement of the beam due to the angular rotation, and $z_{\text {bend }}$ is the displacement due to bending.

Figure 6.3 shows the force trace from the experiment pictured in Fig. 6.2. The blue trace shows the force signal from the QPD while the red trace shows a control measurement performed by sinusoidally driving an unattached bead near the sheet. As can be seen, there is a non-zero signal in the control measurement. This is due to scattering from the polycarbonate membrane (PCM) that the cell sheet is grown on. To determine the flexural rigidity, EI, we take the peak force, maximum displacement, and cilium length from the QPD signal/movies and plug them into Eqn. 6.2. The last two quantities are measured by hand using ImageJ with a calibrated pixel scale. We subtract the peak value of the control measurement from the peak force value to get the "actual" force value, $F$, used in Eqn. 6.2. The trap stiffness and conversion factor, used to calibrate the force measurement, are both determined using the power spectrum method discussed in Chapter 2. Briefly, the bead is moved to the appropriate height in the chamber and a short (10 s) power spectrum at $100 \mathrm{kHz}$ is recorded.

Table 6.1 tabulates $E I$ values from four different cilium-bead constructs from four different sample preparations. There are two types of $E I$ values listed in Table 6.1, values calculated assuming the boundary condition (BC) is fixed ("EI non-adj.") and values calculated assuming a limited hinge BC ("EI adj."). As the reader will see in subsequent sections, the $\mathrm{BC}$ of the cilium attachment point appears not to be clamped (i.e. attachment point position and its first derivative with respect to position are constant in time), which is one of the assumptions in Eqn. 6.2. While 

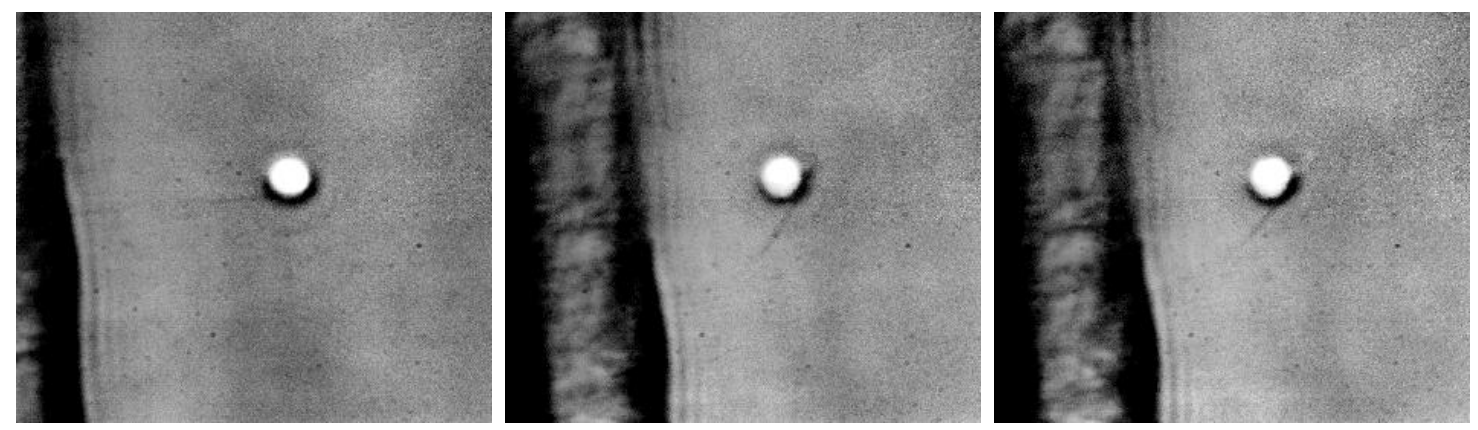

Figure 6.5: Series of stills from an adaptation experiment. An initially unbent cilium is bent with a sudden $x y$ jump of the piezo stage and left in a bent position while the force trace is monitored.

in the last two measurements a clamped $\mathrm{BC}$ appears to be at least approximately valid, in the first two, the cilium hinges through a noticeable angle before it starts to bend. In order to correct for this, we measure the angle, $\theta$, that it rotates through before it starts to bend, again by hand, using ImageJ. These values are given in the first row of Table 6.1. The displacement traveled perpendicular to the original cilium position before bending, $z_{\theta}$, is then given by $z_{\theta}=L \sin \theta$, where $L$ is the distance to the attachment point (usually the length of the cilium). The "real" bending displacement that the cilium goes through is then given by $z_{\text {bend }}=$ $z(L)-z_{\theta}$, where $z$ is the total displacement from the original position, as shown in Figure 6.4. Substituting $z_{\text {bend }}$ into Eqn. 6.2 gives us the second row of $E I$ values in Table 6.1. The averages of the bending stiffnesses quoted in the middle and bottom row are $1.9 \pm 2.0 \times 10^{-23} \mathrm{~N} \cdot \mathrm{m}^{2}$ and $2.5 \pm 1.5 \times 10^{-23} \mathrm{~N} \cdot \mathrm{m}^{2}$ respectively. With this correction the measured flexural rigidity values cluster more tightly together rather than scattering over an order of magnitude. The values reported here are in good agreement with several previous measurements of cilium flexural rigidity using flow $[17,19]$.

Table 6.1: $E I$ values from active bending experiments

\begin{tabular}{|c|c|c|c|c|}
\hline & Cilium 1 & Cilium 2 & Cilium 3 & Cilium 4 \\
\hline$\theta$ (deg.) & $13^{\circ}$ & $20^{\circ}$ & $0^{\circ}$ & $0^{\circ}$ \\
\hline$E I$, non-adj.( $\left.\mathrm{x} 10^{-23} \mathrm{~N} \cdot \mathrm{m}^{2}\right)$ & $0.5 \pm 2.0$ & $0.3 \pm 2.0$ & $4.7 \pm 2.0$ & $1.9 \pm 2.0$ \\
\hline$E I$, adj. ( $\left.10^{-23} \mathrm{~N} \cdot \mathrm{m}^{2}\right)$ & $1.4 \pm 1.5$ & $2.0 \pm 1.5$ & $4.7 \pm 1.5$ & $1.9 \pm 1.5$ \\
\hline
\end{tabular}




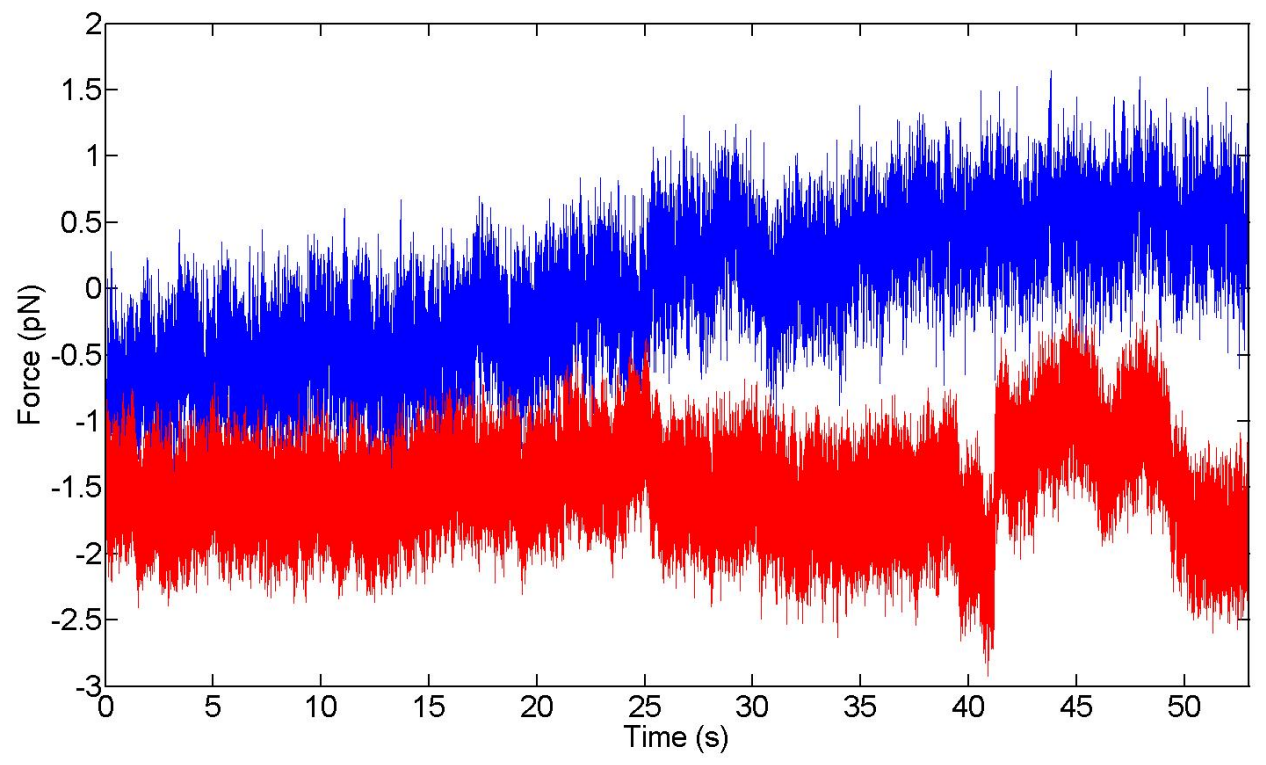

Figure 6.6: Force traces from two adaptation experiments. Both traces are taken from the $x$-channel and start immediately after the $x y$ jump. The blue trace corresponds to the experiment pictured in Fig. 6.5. The abrupt jump around second 40 in the red trace corresponds to debris from solution falling into the trap.

\section{Adaptation experiments}

In addition to measuring the flexural rigidity of primary cilia with cilium attached beads, we also looked at their behavior under a constant, prolonged force. We were curious as to whether we could measure signs of adaptation, such as increasing force due to mechanical feedback from the cell or relaxation of force due to cellular rearrangement. Figure 6.5 shows stills from one such experiment, where a cilium was bent through a large angle by stepping the piezo simultaneously in $x$ and $y$ coordinates (east-west and north-south) by $5 \mu \mathrm{m}$. The stage is left in the post-step position for the duration of the experiment and we record a movie and the QPD signal for roughly a minute. Force curves from two such experiments are shown in Figure 6.6, with the blue trace corresponding to the experiment pictured in Fig. 6.5. Though there is gradual increase in the force over time in the blue curve, the red curve stays essentially flat. Throughout the time course debris from solution falls into the trap, which leads to noise in the force signal and possibly to the overall increase in the blue curve. Given the difficulty of ascertaining whether the signal is due to debris or to forces exerted by the cilium, interpretation of the data is problematic and more measurements are needed to say anything conclusive.

It is worth noting, though, that in the movie one can see relaxation of the initially 

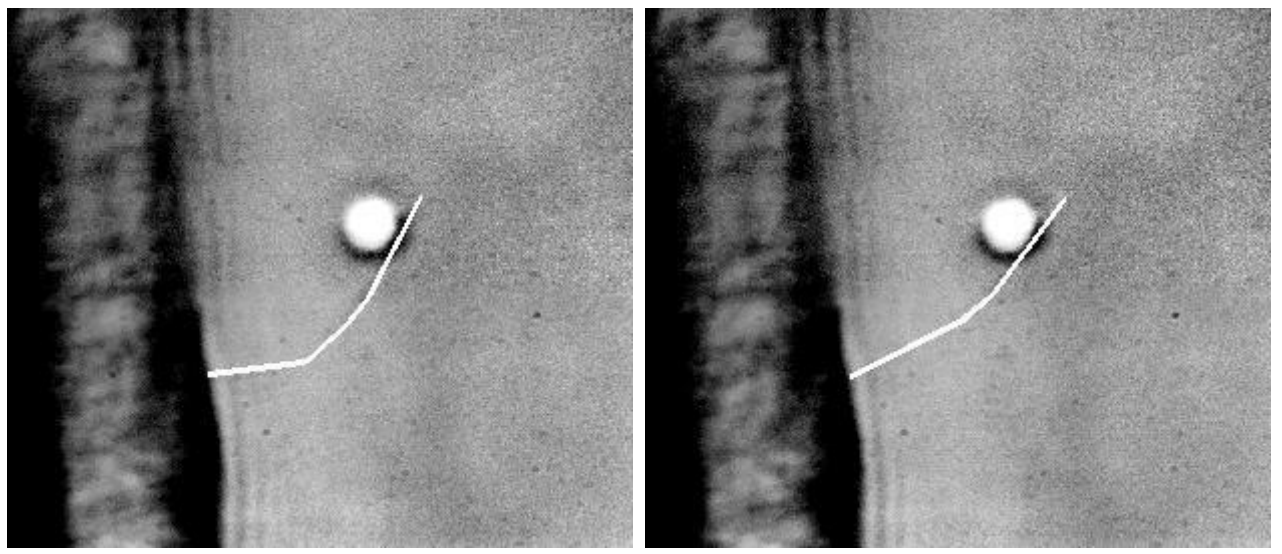

Figure 6.7: Middle and right-hand stills from Fig. 6.5 with the cilium backbone traced in white. There is a relaxation of the bent shape of the cilium over time, with the most noticeable relaxation occurring between seconds 20 and 30 of the blue trace in Fig. 6.6.
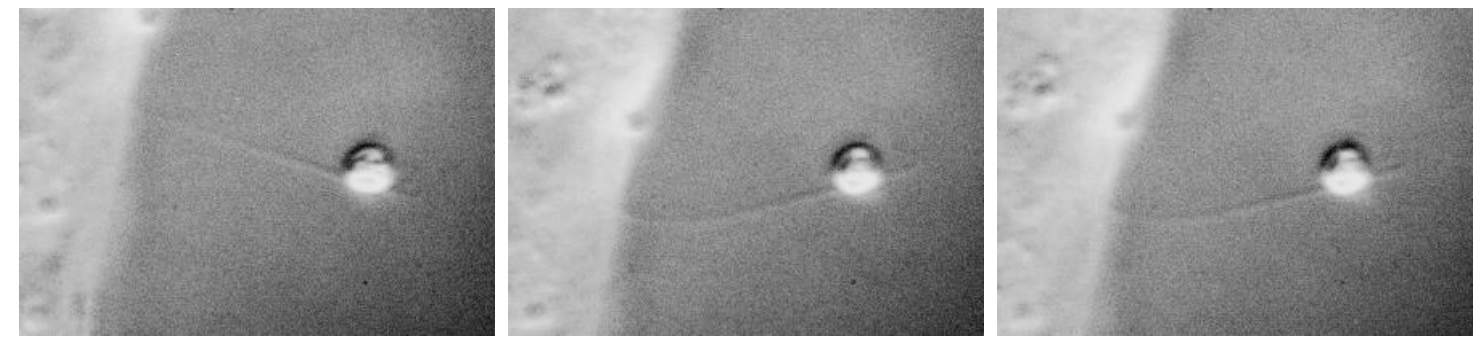

Figure 6.8: Stills from a second adaptation experiment. In this experiment we made a sudden jump in the $+y$ direction (north), which bends the cilium, and the stage was left stationary while monitoring the force trace.

bent cilium curvature by eye, though it doesn't appear in the force measurement. Over a ten second period, the cilium relaxes from its initial, higher curvature shape to a more gently bent curve. Figure 6.7 shows a hand-drawn backbone overlaid on the middle and right-hand frames of Fig. 6.5, which show the cilium before and after the relaxation event respectively. It appears that the initial $90^{\circ}$ bend at the base relaxes by rotating through an angle of $\sim 15^{\circ}$, but the cilium is not well-resolved close to the base, so it's difficult to assert with certainty that this is the relaxation pathway the cilium follows. A bead in an optical trap is free to rotate, so it is also possible that the cilium relaxes out of frame via a rotation of the bead. Given our subsequent findings that the cilium attachment to the cell is better described by a limited hinge BC rather than a clamped one, however, it doesn't seem far-fetched that the cilium could be relaxing tension via hinging at the base. 


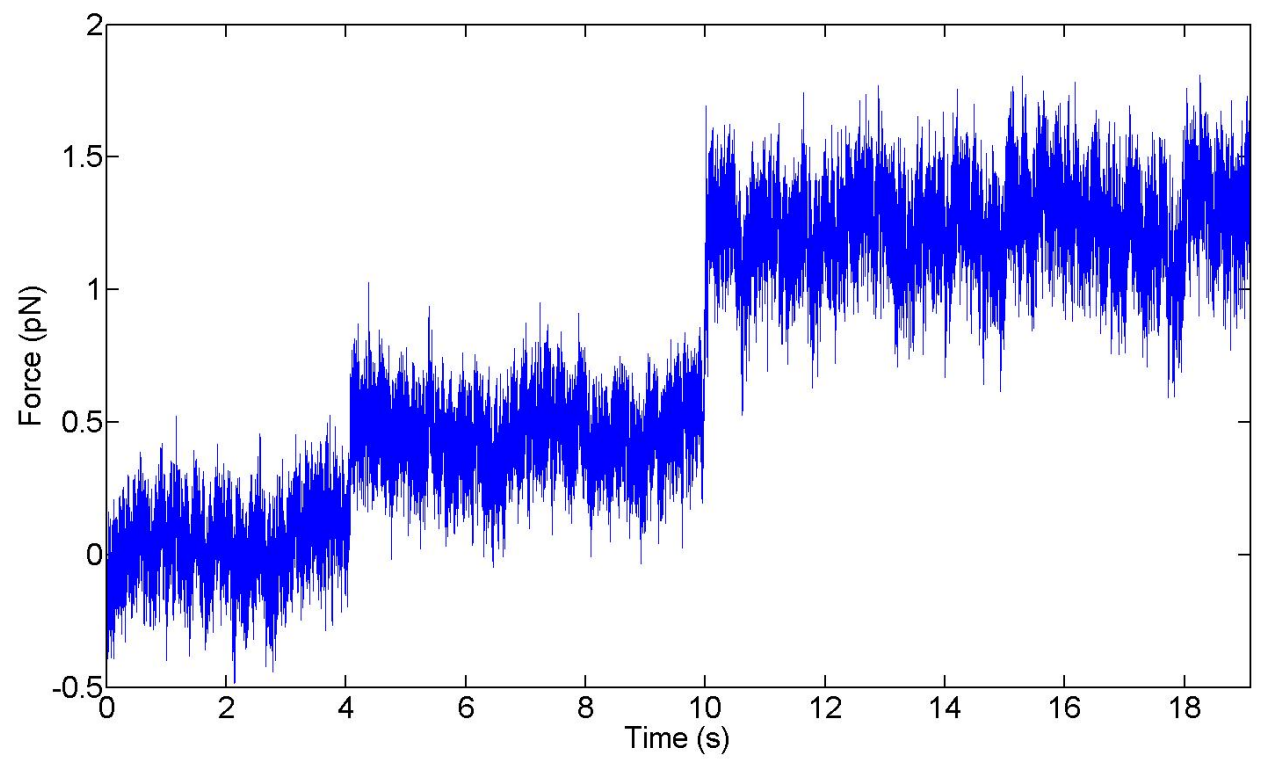

Figure 6.9: Force trace from the experiment depicted in Fig. 6.8. The trace starts immediately after the $y$ jump. While there appear to be step-like increases in force, the jump at second 10 correlates with debris falling into the trap. The earlier jump at second 4 also seems to correlate with debris, though it's more difficult to tell.

We also performed a variant on the adaptation experiment described above in which we stepped the cilium through a $y$ displacement of $+2.5 \mu \mathrm{m}$, watched the time course for $\sim 20 \mathrm{~s}$, stepped the bead back to its initial position, then performed a second $y$ displacement of $-2.5 \mu \mathrm{m}$ and again watched the time course for $\sim 20 \mathrm{~s}$. Figure $6.8 \& 6.9$ show a series of stills from the $+y$ displacement and the associated force trace. The QPD signal shows step-like increases but the second, larger step correlates with debris falling into the trap from solution. The first step also seems to be correlated with debris falling into the trap, though it's more difficult to say. All in all the force trace seems to be dominated by noise and says nothing conclusive about cilium adaptation dynamics.

Figure $6.10 \& 6.11$ show a series of stills and the associated force trace from the subsequent $-y$ displacement in the same experiment. In this case there is a clear decay in the force on the order of a piconewton, which is well-fit by an exponential decay with a time constant of $\tau=2.5$ seconds. The source of this relaxation is unclear. The step may be exerting tension on the cilium due to its initial, non-normal orientation with the $y$-axis and the decay could be related to tension relaxation. Interestingly, between the middle and right frames of Fig. 6.10 the cilium proceeds from a relatively straight conformation to a slightly bent one. 

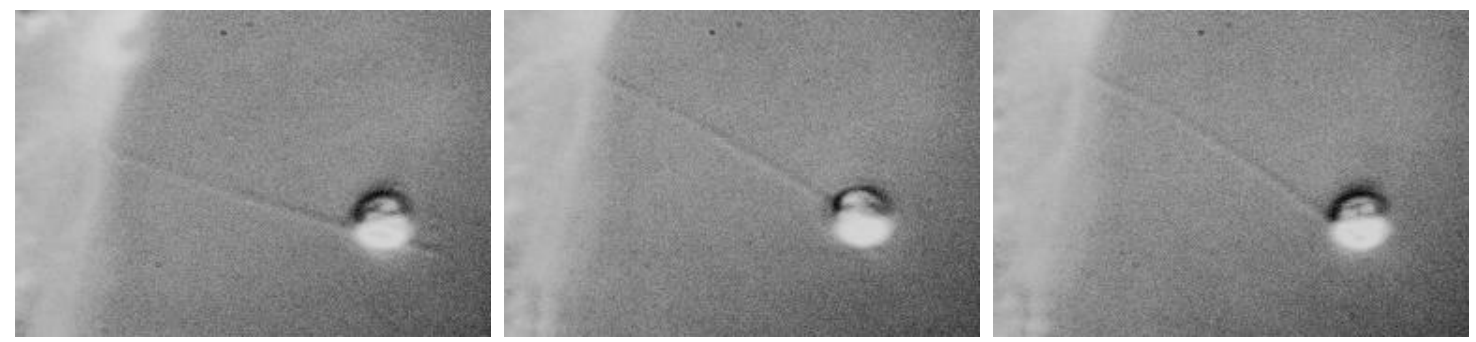

Figure 6.10: Stills from a second adaptation experiment. In this experiment we made a sudden jump in the $-y$ direction (south) and the stage was left stationary while monitoring the force trace. In this case the cilium is not bent as strongly as in Fig. 6.8, due to the cilium initial position (left-hand frame).

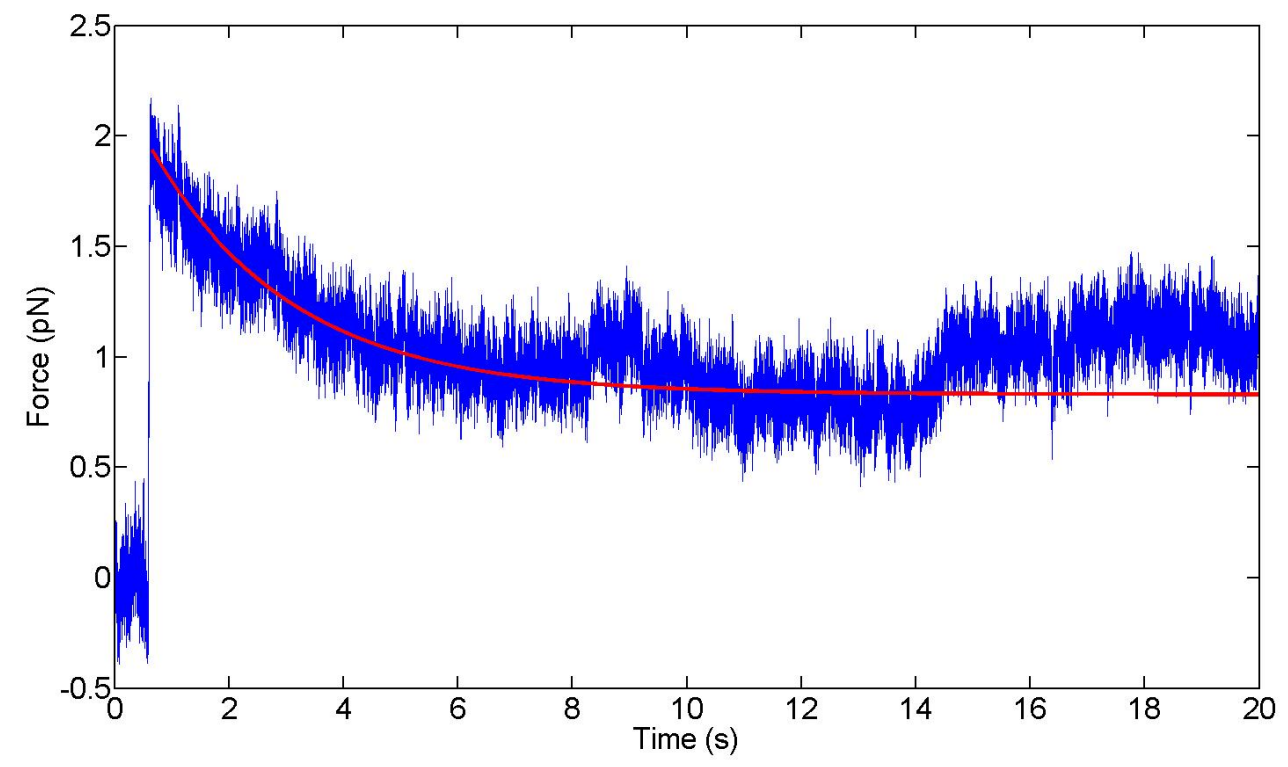

Figure 6.11: Force trace from the experiment depicted in Fig. 6.10. The trace starts just before the $y$ jump (sharp jump at the beginning of the trace). There appears to be an exponential decay in the force immediately after the jump. The red line is an exponential fit with a time constant of $\tau=2.5$ seconds.

This is, however, not the source of the decay that we see; this relaxation occurs in two discrete steps starting about five seconds after the QPD measurement ends.

Figure 6.12 shows a detail of the bead and cilium attachment point before and after the relaxation event. During the relaxation, the bead rotates and moves laterally, as can be seen from its displacement from its initial position (marked by the white circle). Since the relaxation proceeds by two discrete steps, it could be due to breakage 

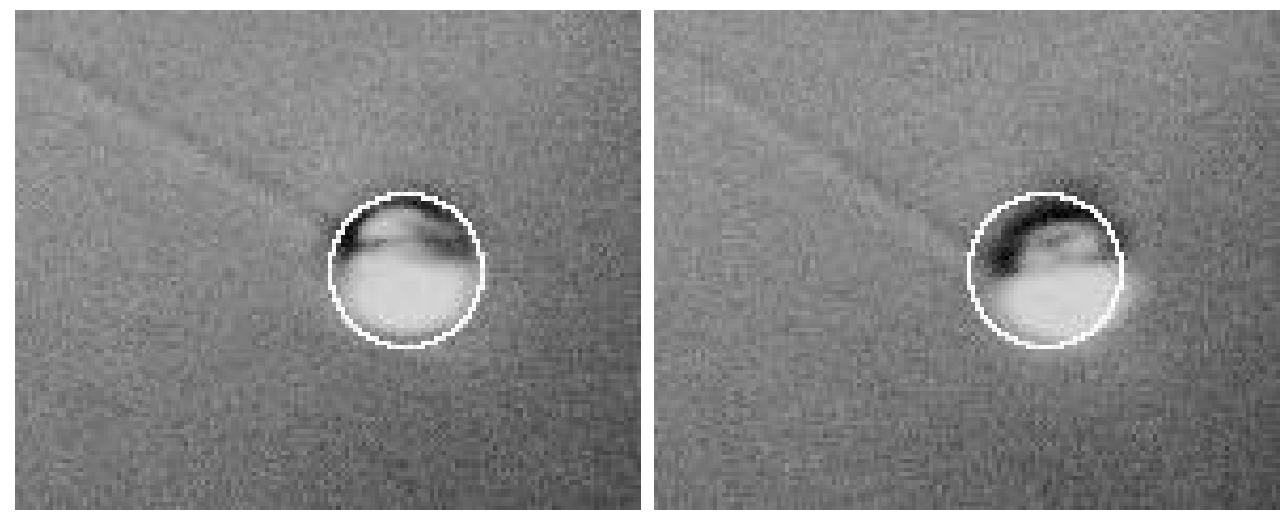

Figure 6.12: Detail of the middle and right-hand stills from Fig. 6.10. The initial bead position after the $-y$ jump is outlined in white. Approximately 30 seconds after the jump there is a quick relaxation, over 3 seconds, in which the bead rotates and the cilium deforms along its length. Unfortunately this event happened 5 seconds after the end of the QPD recording so we have no associated force data.

of bonds attaching the cilium to the bead, perhaps relaxing tension caused by an occluded kink at the attachment point. Taken together, the findings from our adaptation experiments point towards possibly interesting cilium relaxation dynamics, but more experiments are needed to disentangle the processes underlying them.

\section{Bend \& relax experiments}

In order to measure the bending stiffness of the primary cilium by a different route, we performed a second type of experiment based on a technique previously used to measure microtubule flexural rigidity [78]. In these experiments we displaced the cilium from its initial position with the optical trap (no bead attached), by either moving the piezo stage or the piezo mirror, and watched the time course of the cilium relaxation to its initial position after release from the trap. Images were acquired at a rate of $25 \mathrm{~Hz}$. Figure 6.13 shows stills from one such experiment.

Since the cilium dynamics occur at a low Reynolds number $\left(\approx 10^{-6}[79]\right)$, the inertial forces acting on it are negligible and viscous drag dominates. In this case the elastic restoring force of the rod is in a force balance with viscous drag, leading to exponential decay dynamics of the cilium tip position. For an isotropic, homogeneous filament clamped at one end with its tip displaced a perpendicular distance $z(L)$ at time $t=0$ (see sketch in Fig. 6.1, with $F=0$ at $t=0$ ), the tip position as a function of time, $z(L, t)$, is given by [79]

$$
z(L, t) \simeq z(L, 0) e^{-r t}
$$



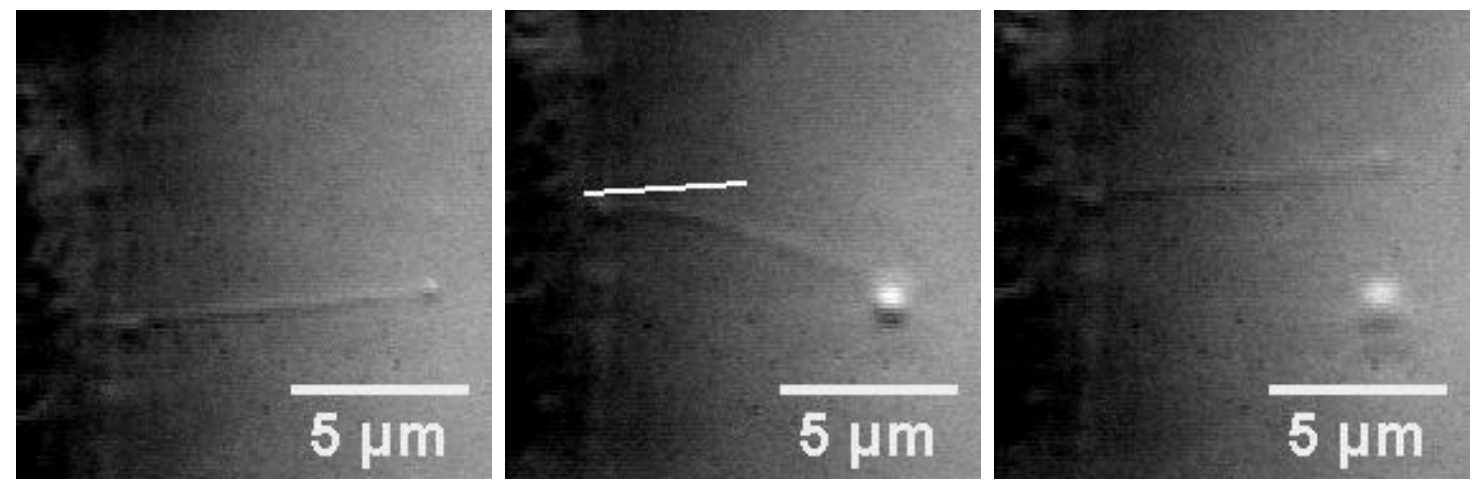

Figure 6.13: Series of stills from a bend \& relax experiment. From left to right, the initially unperturbed cilium is bent using an optical trap and then released from the trap to relax back to its initial position. The white line in the middle frame indicates the cilium initial position. Note that there is no bead involved in these experiments; the white ball that follows the trap is debris from the solution which has fallen into the trap.

where

$$
r=\frac{E I k_{1}^{4}}{\zeta L^{4}}
$$

and $L$ is the length of the cilium, $\zeta$ is the viscous drag coefficient of the cilium, and $k_{1}=\pi / 2+0.304$ is the first solution to the transcendental equation $\cos k \cosh k=$ -1 . The number $k_{1}$ is a coefficient associated with the lowest order mode in the family of solutions to the equations of motion, and it is this mode that dominates the long-time decay of the filament to its original position (the other modes contribute less than 3\%) [79]. The viscous drag coefficient of the cilium is given by

$$
\zeta=\frac{4 \pi \mu}{\ln (L / d)+c}
$$

where $\mu$ is the dynamic viscosity of the fluid (here we use the dynamic viscosity of water, $\mu \approx 10^{-3} \mathrm{~Pa} \cdot \mathrm{s}$ ), $d$ is the diameter of the cilium (we assume $d=250 \mathrm{~nm}$ ), and $c$ is a constant depending on the body's shape, of order unity (we assume $c=1$ ) [79]. It should be noted that this approximation relies upon the displacement $z(L, 0)$ being small (low radius of curvature) so that the bending moment of the filament, $M$, can be approximated as $d^{2} z / d x^{2}$. Following the treatment in Ref. [78], we limited deflections of the cilium to less than $45 \%$ of the cilium length. With the relevant mathematics in hand we can now proceed to extract the bending stiffness from the overdamped cilium tip decays, needing only the cilium length and the tip position at each time point. The cilium tip is tracked frame-to-frame by hand. Figure 6.14 


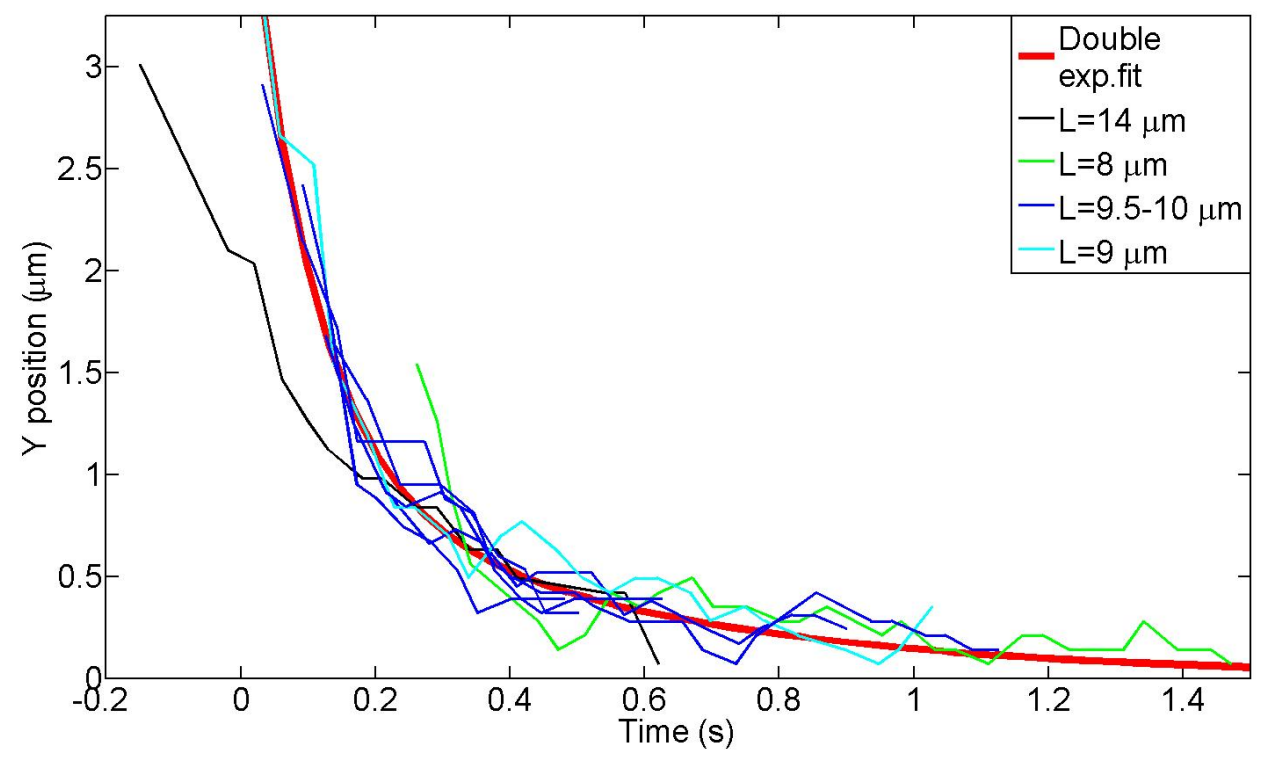

Figure 6.14: Relaxation time curves for cilia of varying lengths. All curves exhibit biexponential relaxation behavior and longer cilia have a slower exponential folding time, as expected. The thick red line is a double exponential fit to the average of the cilia from 9-10 $\mu \mathrm{m}$ long.

shows 8 curves from cilia of lengths varying from $8-14 \mu \mathrm{m}$ from 4 different sample preparations. While we took data from shorter cilia as well, we had to discard them due to their extremely fast relaxation time. In essence they relaxed from one frame to another, so the data were unusable.

Cilia of different lengths relaxed at different speeds, as expected from the quartic length dependence of Eqn. 6.4. Interestingly though, rather than following a single exponential decay as expected, the relaxation curves exhibited double exponential decay dynamics. Reviewing the movies frame by frame, one notes that the curvature relaxes within a maximum of 8 frames $(320 \mathrm{~ms})$ followed by a slower, sometimes "stop-and-go" movement of the straightened cilium back to its initial position. The slower exponential relaxation might correspond to a relaxation of the rotational/translational degrees of freedom of the cilium, to be discussed in the next section. As the curvature clearly relaxes on the faster of the double exponential time scales, we took this to be the relevant time scale for determination of the flexural rigidity. Fitting the various curves in Fig. 6.14 and plugging the faster time constants into Eqn. 6.3 yields a bending stiffness of $E I=3.6 \pm 0.8 \times 10^{-23} \mathrm{~N} \cdot \mathrm{m}^{2}$. This is of the same order of magnitude as the results from our active bending measurements and similar to literature values $[17,19]$. 


\section{Errors}

The main sources of error in the above experiments came from the ubiquitous debris in solution, scattering from the PCM, and difficulties in force calibration. Readers familiar with micromechanical measurements of biopolymers will no doubt wonder why we chose to measure the bending stiffness in the active bending experiments in the way that we did rather than by buckling the cilium with compressive loading. In this case the bending stiffness would be given by $E I=F_{\text {buckle }} L^{2} / \pi^{2}$, where $F_{\text {buckle }}$ is the threshold buckling force [74]. Initially we tried this approach, but we realized that the photodiode signal was dominated by scattering from the sheet and we couldn't discriminate bead signal from the spurious PCM response. Even moving the trap in the $y$-plane led to some spurious signal (see Fig. 6.3), likely due to the PCMs imperfect alignment with the $y$-axis. Scattering from the PCM may have also played a role in the poor power spectral calibration of some beads, though we didn't investigate this thoroughly. Often the power spectra used to calibrate our beads contained large amounts of low-frequency noise This problem was compounded by the fact that we worked at heights in the chamber of $\sim 70 \mu \mathrm{m}$, where the trap is weaker due to the refractive index mismatch between the oil-immersion objective and the aqueous solution. The combination of low-frequency noise and a weak trap can wipe out the characteristic Lorentzian plateau and make accurate curve fitting, necessary for accurate force calibration, difficult.

The debris in solution is a problem that we tried to overcome by flushing our samples with fresh solution prior to starting our experiments, as described in the Experimental methods chapter. When effective, this generally gave us a window of 10 minutes in which to perform our experiments before the debris started to appear in the field-of-view again. The debris itself probably consists of cellular matter shed by the monolayer (apoptosed cells, membrane vesicles, vesicles shed from cilia), though some of it may be pieces of the monolayer damaged from the image chamber mounting process. The debris makes long-term force measurements, such as the adaptation experiments, difficult to interpret as it perturbs the signal unpredictably upon falling into the trap. It is also the probable culprit for the aforementioned low-frequency noise. Our experiments could possibly be improved by designing new imaging chambers with a thin glass wall for the cells to grow on, along with stably attached inlet/outlet adapters. The cells could then be cultured inside the chamber turned on its side, so that they grow to confluence on the wall rather than the bottom. In this configuration there should be less scattering from the substrate that the cells are attached to, and the user would have the possibility to repeatedly flush the chamber with fresh solution to mitigate the debris problem. 

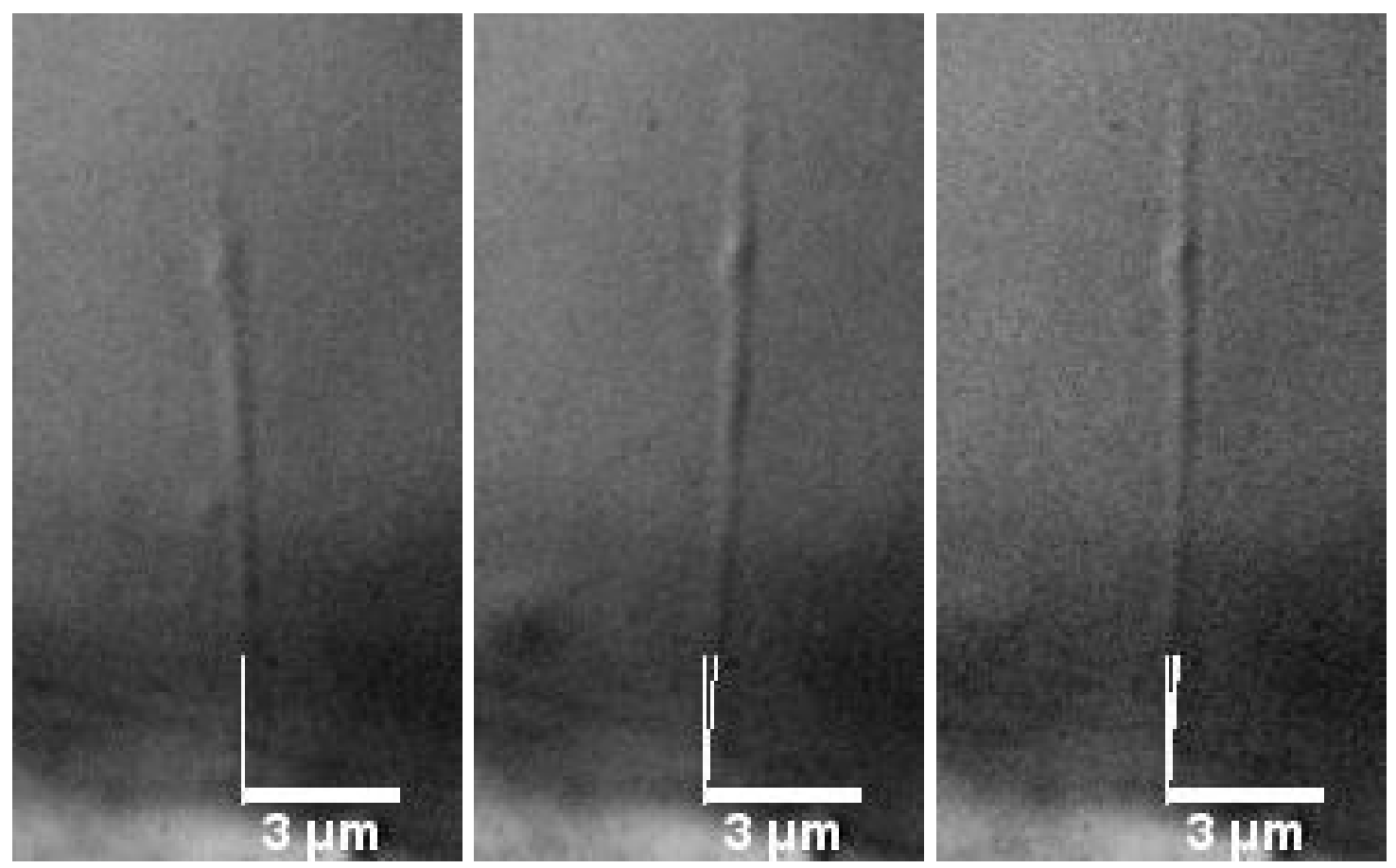

Figure 6.15: Stills from a fluctuation analysis movie. The white lines at the base mark the position of the cilium in each of the stills, as well its position in the preceding stills.

\section{Fluctuation analysis}

When primary cilia are viewed under a microscope one can see them moving back and forth about an equilibrium position, as shown in Figure 6.15. Primary cilia lack the machinery necessary for motion present in motile cilia and flagella, namely inner and outer arm dyneins, and hence are designated non-motile cilia [11]. Microscopic objects such as the primary cilium are subject to Brownian motion, which arises from the thermal motion of the surrounding solvent. Previous studies on microtubule bending stiffness have exploited thermal fluctuations to probe their mechanics [68, $80,81]$, so we set out to do the same with the primary cilium. The bending stiffness of an isotropic, homogeneous rod clamped at one end and buffeted by equilibrium thermal fluctuations is given by [68]

$$
E I=\frac{k_{B} T l^{3}}{3\left\langle d^{2}\right\rangle}
$$

where $k_{B}$ is Boltzmann's constant, $T$ is the temperature, $l$ is the distance from the clamped end, and $\left\langle d^{2}\right\rangle$ is the mean squared (transverse) deflection of an axis point on the cilium a distance $l$ from the clamped end. Here and in the following 


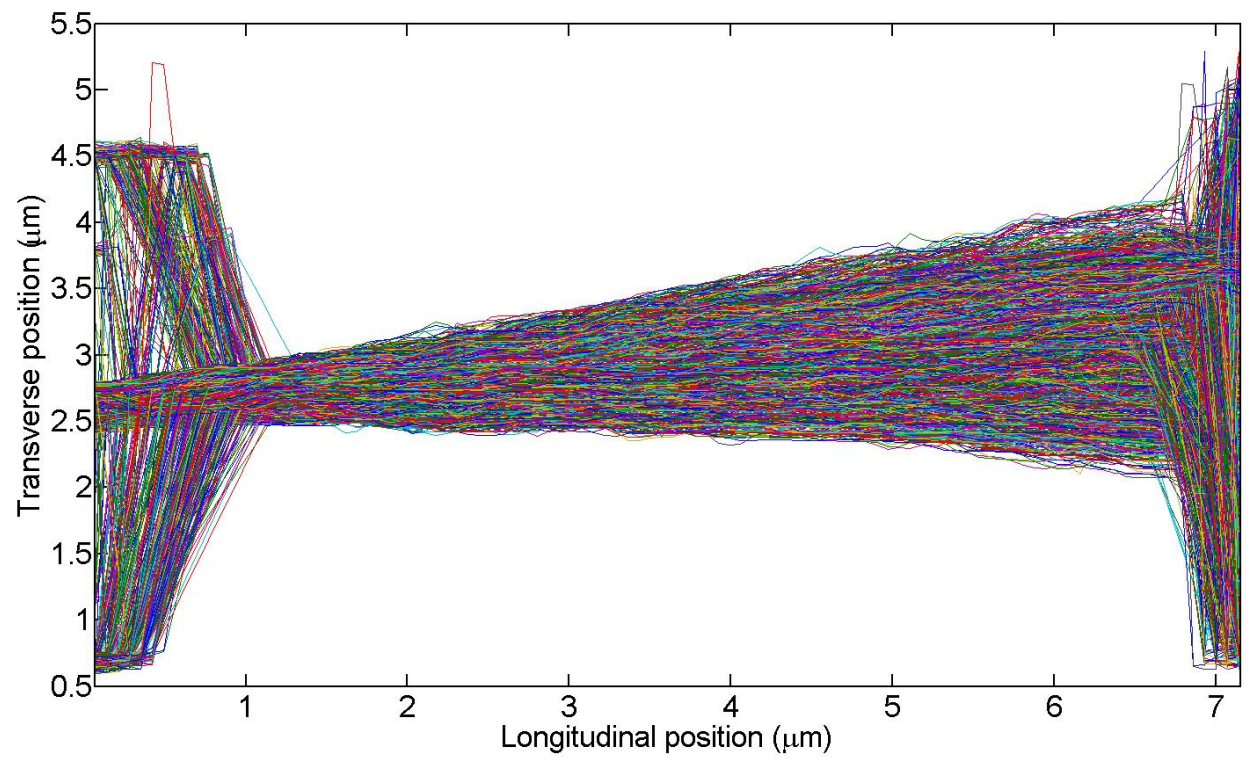

Figure 6.16: Overlay of 6500 cilium backbones. The cilium base is on the left and the tip is on the right. The algorithm fails to accurately track points below about $1.5 \mu \mathrm{m}$ and above $6.5 \mu \mathrm{m}$, so these points are excluded from our analysis.

longitudinal will refer to points along the cilium axis and transverse will refer to points perpendicular to the cilium axis. Given this relation, one can determine $E I$ by recording the position time course of a cilium undergoing thermal fluctuations. To this end we recorded DIC movies of the fluctuations of cilia at $25 \mathrm{~Hz}$ for several minutes (3,000-10,000 frames) and then used the tracking algorithm described in Chapter 4 to extract a cilium backbone from each frame. The graph in Figure 6.16 shows a superimposition of more than 6000 backbones from one such movie.

The cilium sweeps out a straight-edged, "flower vase" shaped envelope in time, suggesting that, rather than bending being the main contributor to motion, as in the case of a clamped rod undergoing thermal fluctuations, the cilium has a rotational degree of freedom similar to a stiff rod on a limited hinge. As is apparent from Figure 6.17, which shows backbone overlays for nine different cilia of differing lengths, this rotational degree of freedom seems to be a common feature among them. Noting this, we attempted to characterize the cilium base attachment $\mathrm{BC}$ quantitatively.

\section{Boundary conditions}

Figure 6.18 shows the transverse fluctuations of three points along a cilium over time. The red trace shows the fluctuations of the lowest point (closest to the base) tracked on the cilium, the blue trace those of the highest point (closest to the tip), 

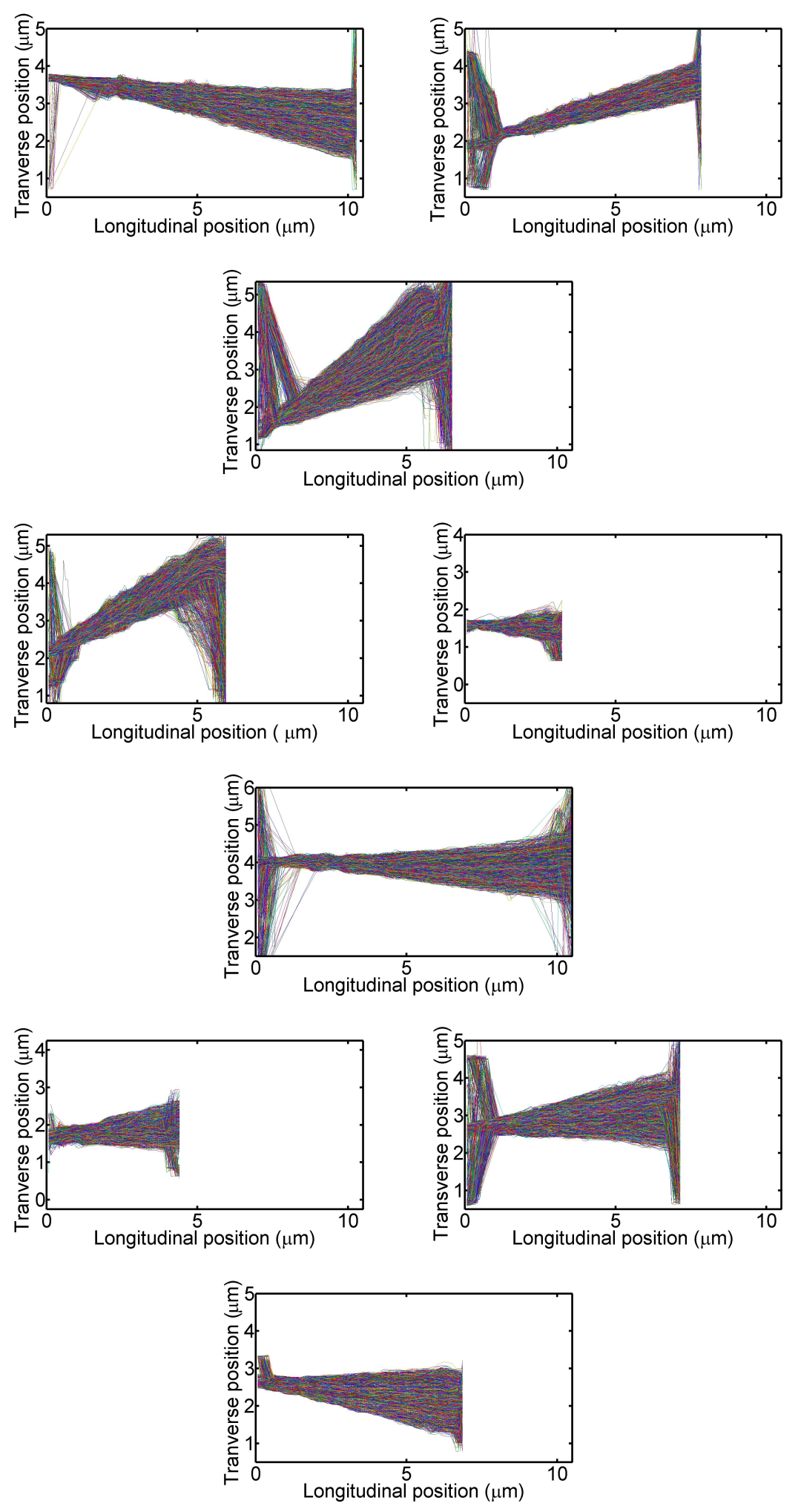

Figure 6.17: Nine plots of superimposed backbones taken from different movies. The axes of the various plots are the same lem to aid comparison of the backbones. 


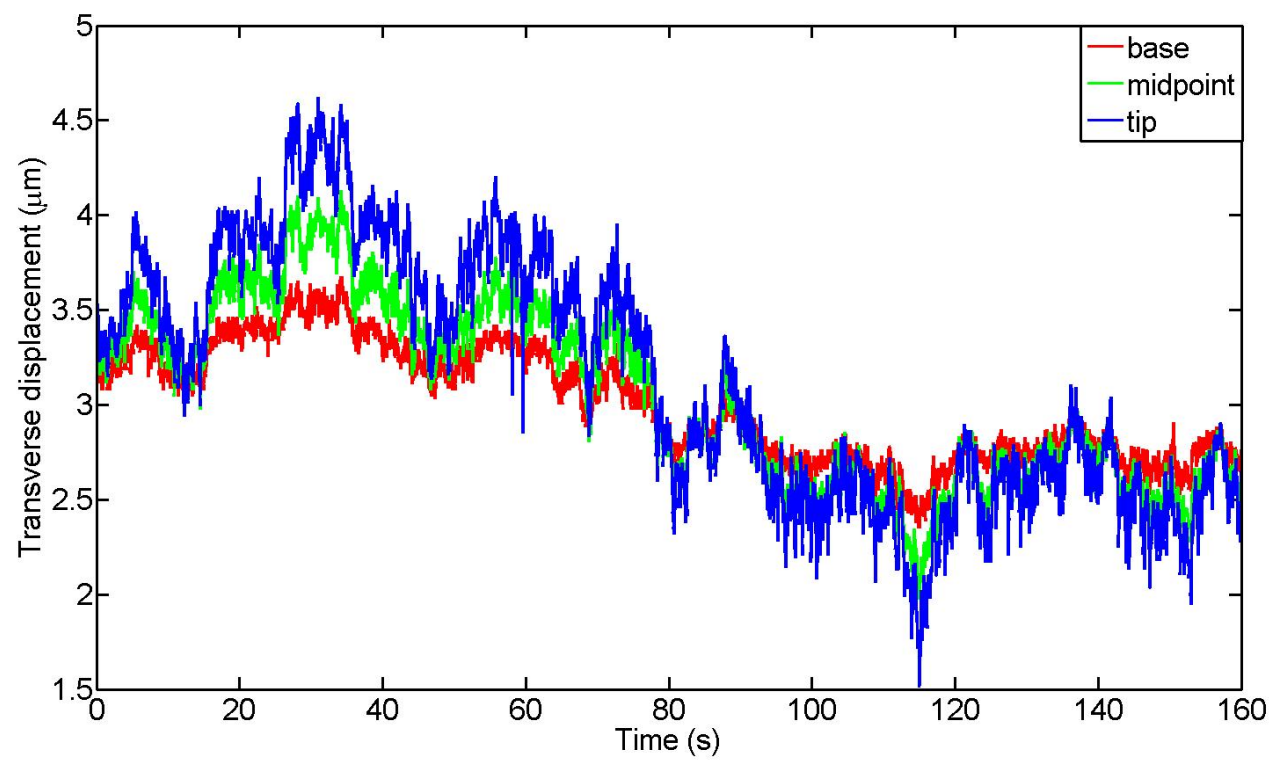

Figure 6.18: Plot of cilium transverse fluctuations. Red encodes the fluctuations of the lowest tracked point, blue those of the highest tracked point, and green those of the midpoint between the two.

and the green trace those of the midpoint between the two. As an example, in the backbone plot shown in Fig. 6.16 the lowest point would correspond to a longitudinal position of roughly $1.5 \mu \mathrm{m}$, the highest point to $6.5 \mu \mathrm{m}$, and the midpoint to $4 \mu \mathrm{m}$. As expected, the fluctuation amplitude increases with increasing distance from the cilium base.

To determine if the cilium was indeed hinging at the base like a stiff rod, we plotted the angle made by each tracked point on the cilium with a centerline extending from the cilium base normal to the cell sheet (in Fig. 6.16 this would correspond to a line running through a transverse position of approximately $2.6 \mu \mathrm{m}$ ). If the cilium is hinging as a stiff rod the angle made by each point with the centerline should be the same. Conversely, curvature in the backbone will show up as a spectrum of angles along the cilium length. Figure 6.19 shows a plot of this angle for the points shown in Fig. 6.18. The angles collapse onto each other convincingly, supporting the hypothesis that the cilium behaves as a stiff rod with a hinged BC (i.e. attachment point position and its second derivative with respect to position are constant in time).

Since the dominant motion seen in the backbone plots and transverse fluctuations is that of a stiff-rod hinging through an angle, we use the mean angle as a measure of overall cilium fluctuations. Figure 6.20 shows the angles made by all the tracked 


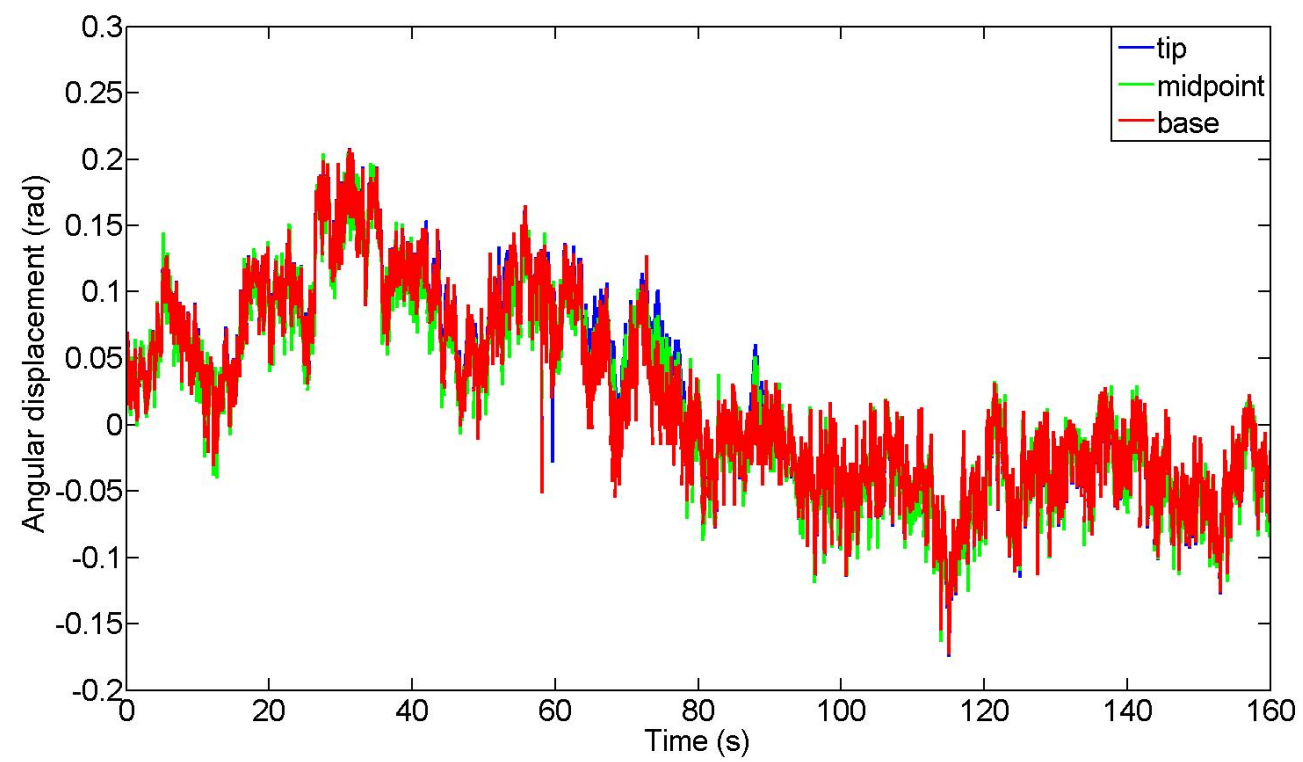

Figure 6.19: Plot of angular fluctuations. The colors correspond to the angles made by the points in Fig. 6.18 with a centerline normal to the cell sheet. The angles collapse onto each other as expected for hinged stiff-rod motion.

points with the centerline in blue with their mean overlaid in red. The mean of the angular standard deviations at each time point, taken as a measure of the "goodnessof-fit" of the mean angle approximation, is $\sigma_{\text {mean }}=0.007 \pm 0.002$ radians $\left(0.4 \pm 0.1^{\circ}\right)$. Thus the spread in angles about the mean angle is small, supporting the claim that the mean angle is a good approximation for the overall cilium motion that we observe.

As a final gauge of the cilium $\mathrm{BC}$, we examined the scaling of the variance of the transverse fluctuations with distance from the cilium base. From Eqn. 6.5 it is clear that transverse bending fluctuations scale with the cube of the distance from the base, i.e. $\left\langle d^{2}\right\rangle \propto l^{3}$. The scaling behavior of a stiff rod on a hinge, however, is qualitatively different. Basic trigonometry dictates that, for a straight rod hinging through an angle $\theta, l \sin \theta=d$, where $d$ is the transverse displacement and $l$ is the distance to the hinge (see Fig. 6.4), with $d=z_{\theta}$. Thus for any given angular rotation, the square of the transverse displacement must scale as the square of the distance to the base, i.e. $\left\langle d^{2}\right\rangle \propto l^{2}$. Figure 6.21 shows the variance of the transverse displacements along the length of the cilium with a quadratic fit. The fit is in good agreement with the data, implying that the scaling is indeed consistent with that of a stiff rod with a hinged attachment point.

Figures 6.22-6.27 depict transverse fluctuation, angular collapse, mean angle, 


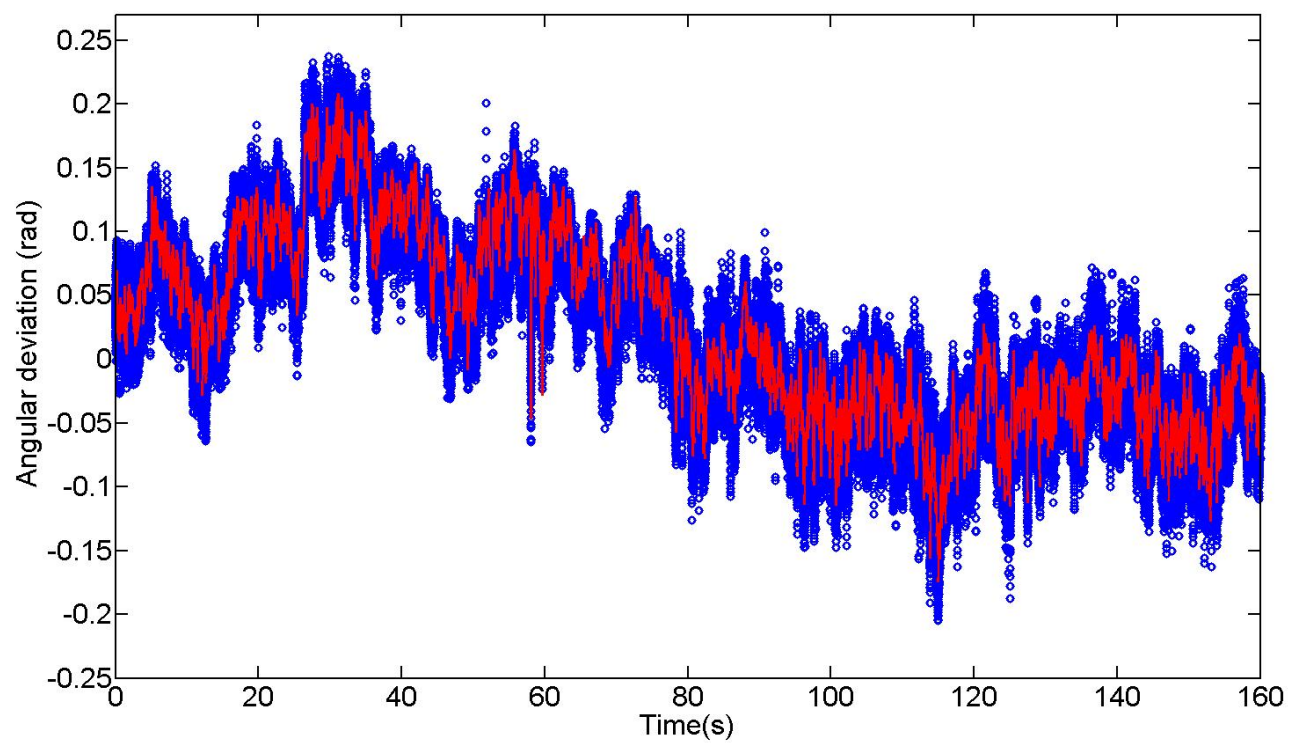

Figure 6.20: Plot of mean angle fluctuations. The data are taken from the same movie as the preceding two graphs. The angles for all the tracked points are plotted in blue with the mean angle overlaid in red.

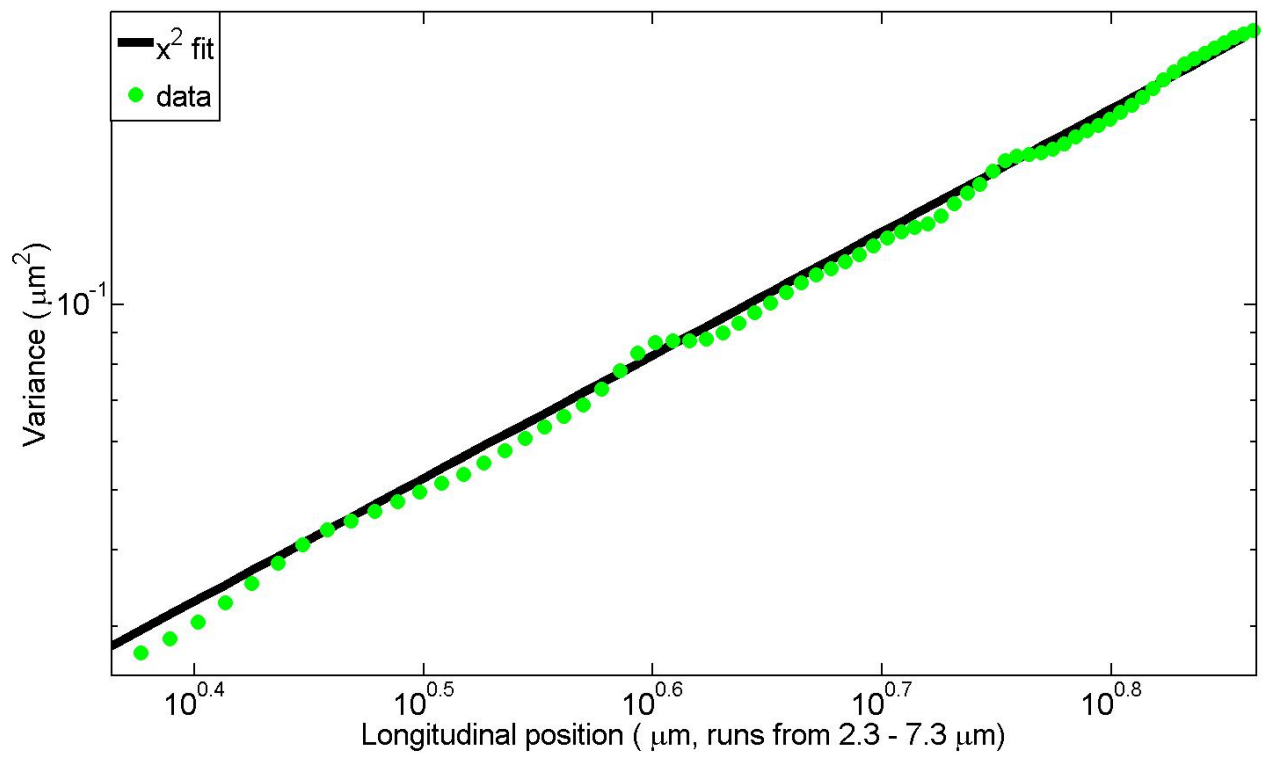

Figure 6.21: Plot of transverse variance vs. longitudinal position. The data are taken from the same movie as the preceding three graphs. The data are plotted as green points with a quadratic fit plotted as a solid black line. 

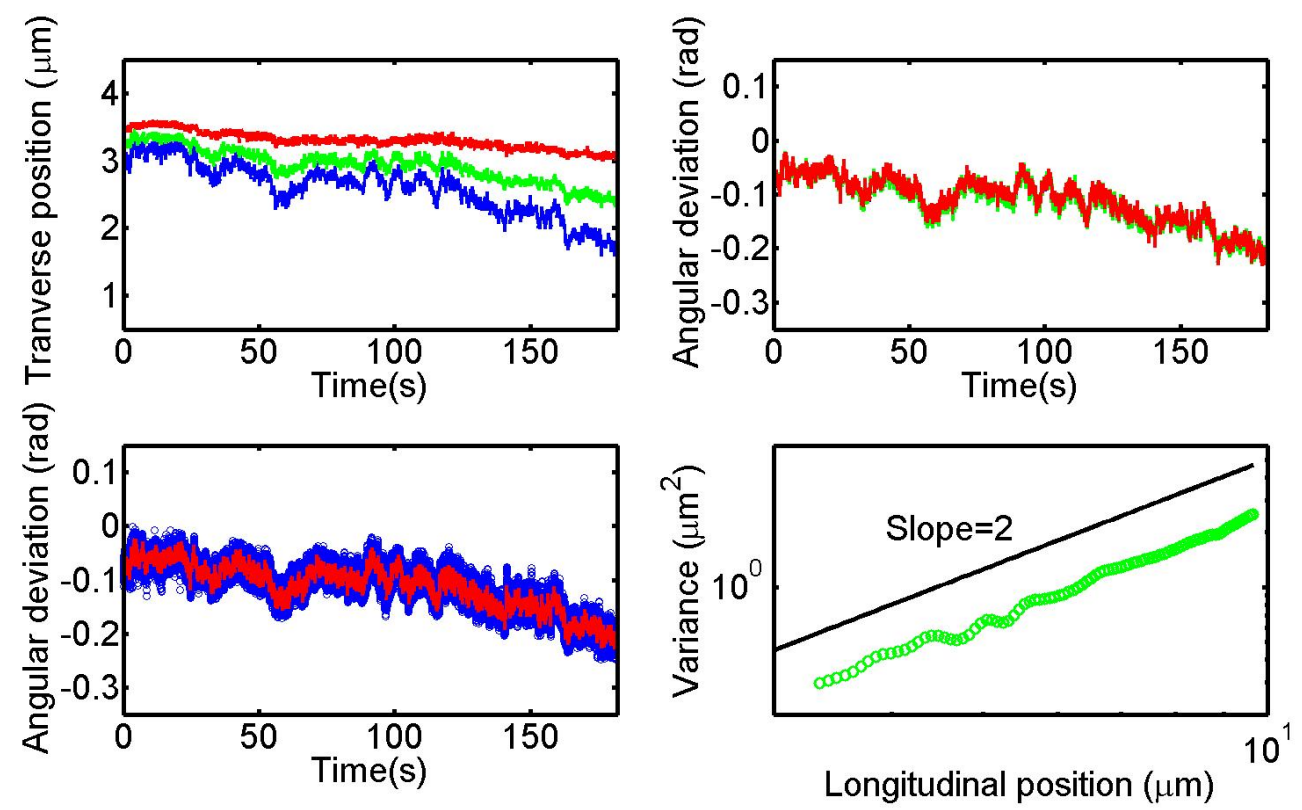

Figure 6.22: Transverse fluctuation, angular collapse, mean angle, and transverse scaling plots for the left-hand cilium in the top row of Fig. 6.17.

and transverse scaling plots for the cilia pictured in the first six (left to right, top to bottom) backbone plots from Fig. 6.17. The ordinate scale for each plot type from figure to figure is kept the same to aid in comparison. The black lines in the transverse scaling plots are all proportional to the square of the abscissa (thus have a slope $=2$ on a log-log plot) but aren't fit to the cilium backbones.

The characteristic angular collapse and stiff-rod scaling holds for the cilia shown and indeed for almost all the cilia that we observed. The angular collapse and scaling is somewhat worse in Fig. 6.26 due to debris diffusing through the frame, which causes a kink in the tracked cilium backbones over some of the time series. For longer cilia $(>10 \mu \mathrm{m})$, however, bending modes become more important and can't be ignored. We don't include any data from such cilia in our analysis because of the difficulty in reliably tracking them on the time scales presented here, over which they tend to defocus and disappear due to out-of-plane bending. The difficulty in tracking longer cilia with clear bending modes and the fact that cilium motion is dominated by stiff-rod hinging unfortunately makes the application of Eqn. 6.5 to determine the flexural rigidity impossible.

The observant reader will have noticed that the attachment point of the cilium to the cell, i.e. the leftmost point in the backbone plots, is not a point but rather a line of points. In Fig. 6.16, for example, the location of the cilium attachment point varies between transverse positions of $2.5-2.8 \mu \mathrm{m}$. This suggests that the hinge 

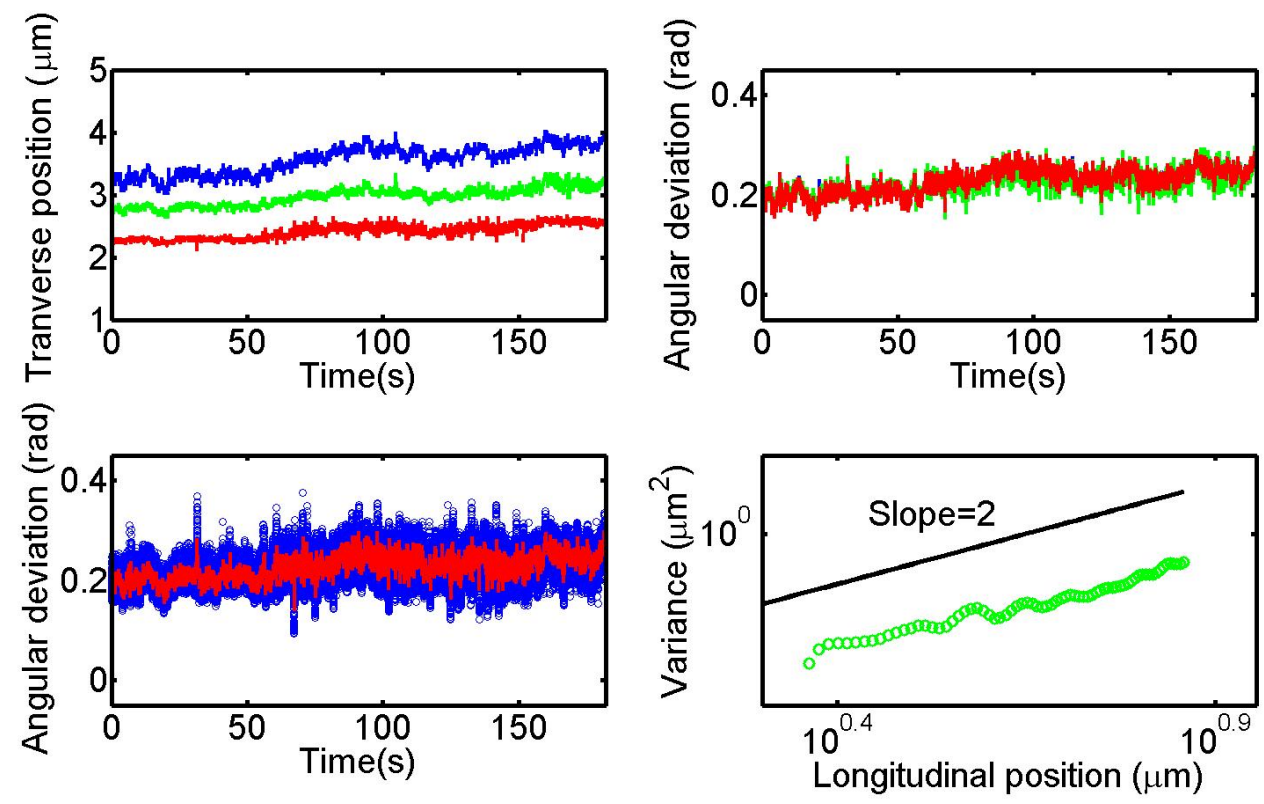

Figure 6.23: Transverse fluctuation, angular collapse, mean angle, and transverse scaling plots for the right-hand cilium in the top row of Fig. 6.17.
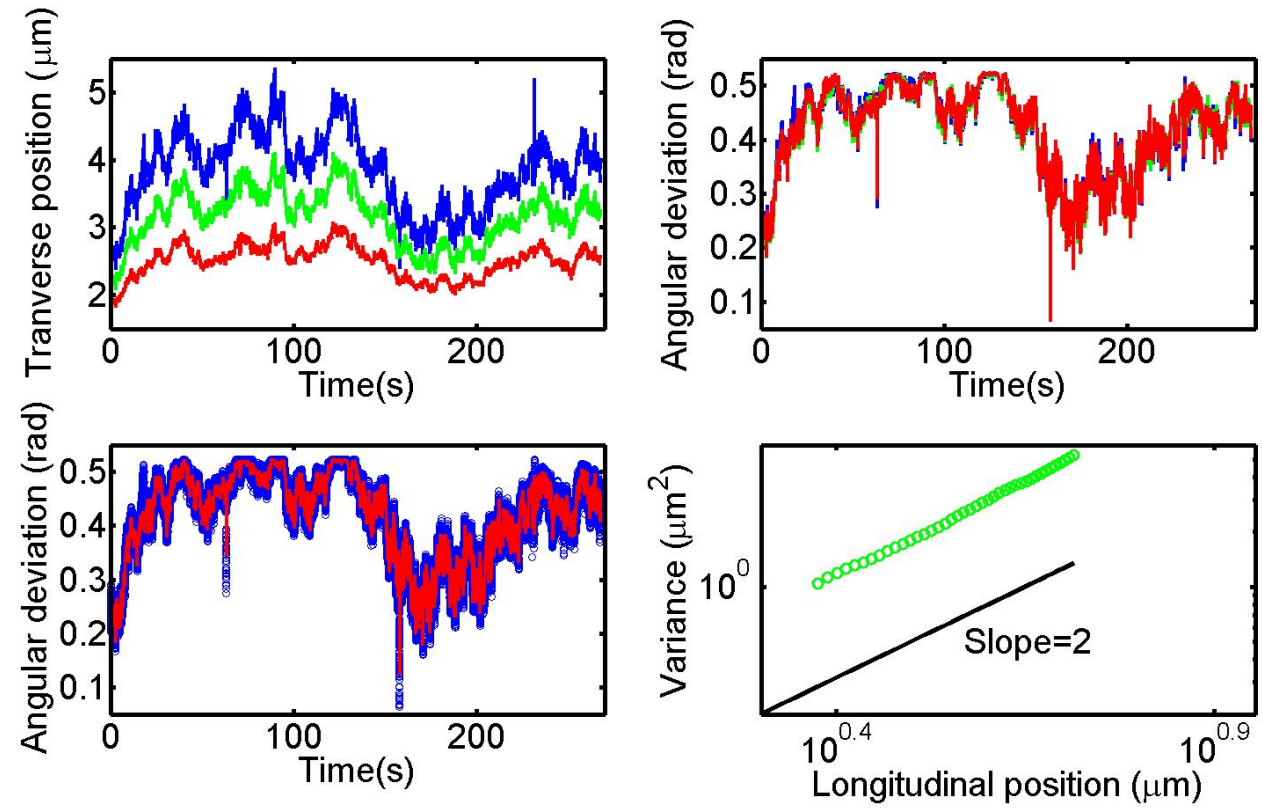

Figure 6.24: Transverse fluctuation, angular collapse, mean angle, and transverse scaling plots for the cilium in the second row of Fig. 6.17. 

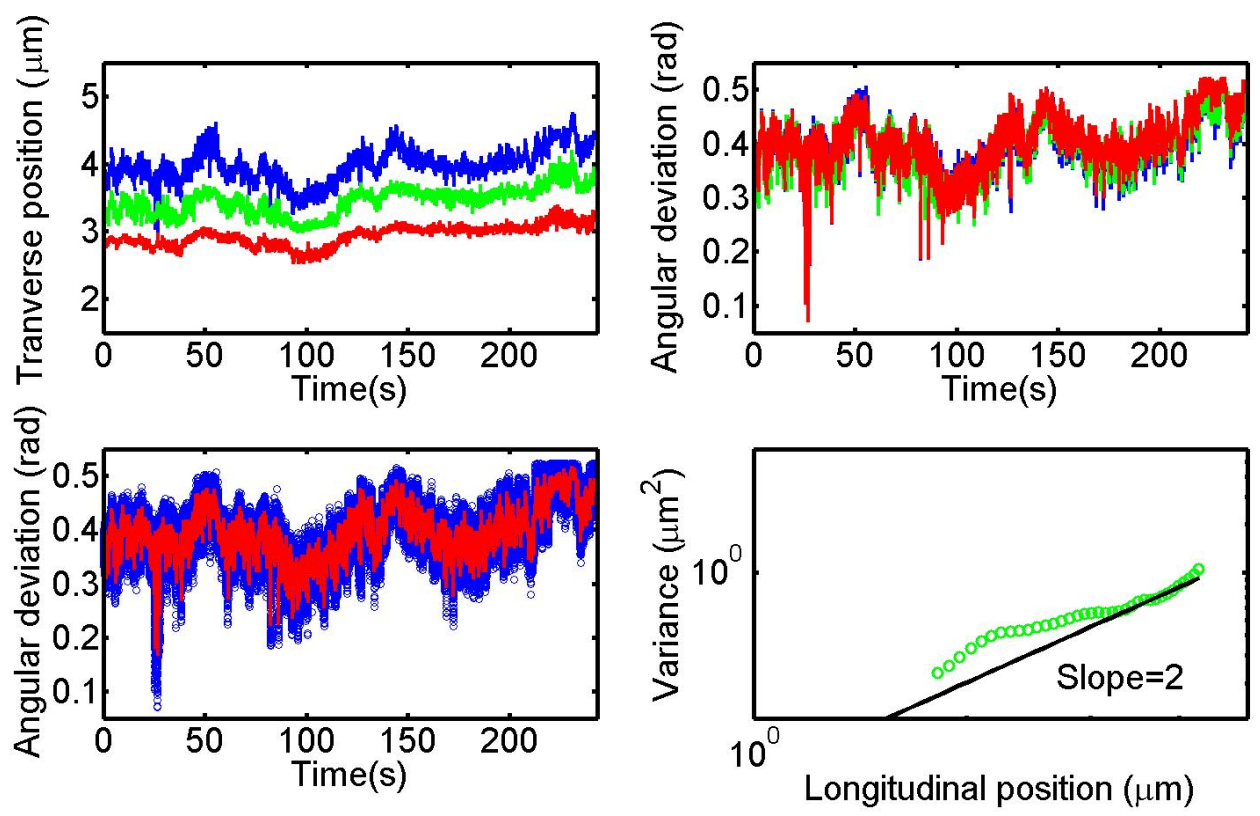

Figure 6.25: Transverse fluctuation, angular collapse, mean angle, and transverse scaling plots for the left-hand cilium in the third row of Fig. 6.17.
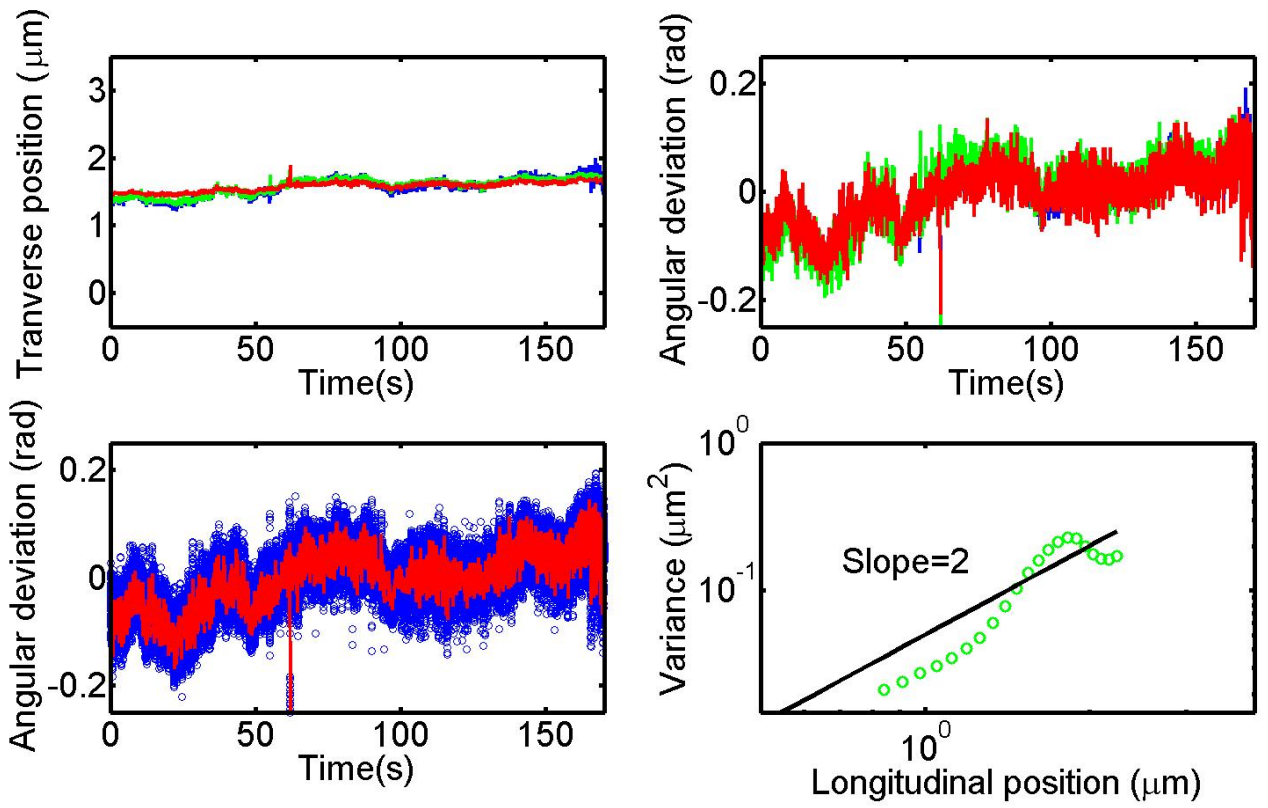

Figure 6.26: Transverse fluctuation, angular collapse, mean angle, and transverse scaling plots for the right-hand cilium in the third row of Fig. 6.17. 

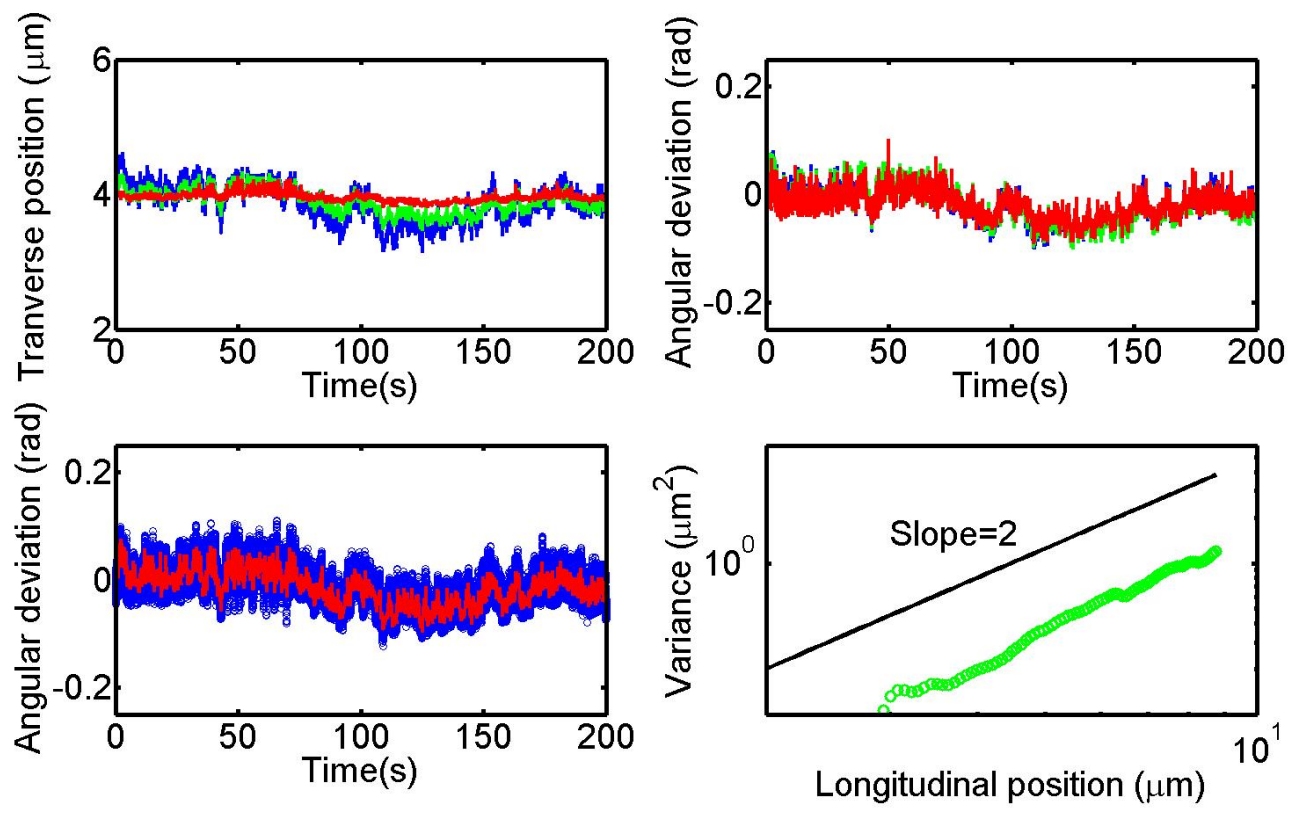

Figure 6.27: Transverse fluctuation, angular collapse, mean angle, and transverse scaling plots for the cilium in the fourth row of Fig. 6.17.

point is below the cell membrane rather than at the membrane. In order to find the hinge point, we extrapolated linearly from the tracked backbones to find where they crossed. In doing this, we averaged backbones in groups of 25 and performed a linear fit to the average. The backbones were averaged in order to smooth out noise and 25 frames was chosen since large-scale cilium movements tend to happen on second or longer timescales. The specific number of backbones averaged had little effect on the result, unless it was an order of magnitude higher ( $\sim 100$ backbones). Figure 6.28 shows an overlay of linear fits (red) on a superimposed backbone plot (blue). The linear fits don't all converge to a single point but rather form a "focus" like a ray bundle in geometric optics. In order to find the focus, i.e. densest bundle cross section, we found the cross section of the bundle with the minimal standard deviation and took this to be the hinge point. This cross section is designated with a black line in Fig. 6.28. Averaging 15 different cilia gave a hinge point position of $-0.2 \pm 0.7 \mu \mathrm{m}$, where a negative value designates a point below the surface. Of these 15 cilia averages, 4 yielded positive values, indicating a hinge point above the surface which was clearly not the case. If we discard these values, the average of the remaining 11 backbones gives a hinge point position of $-0.5 \pm 0.5 \mu \mathrm{m}$.

The hinge point analysis is complicated by the fact that the cilium undergoes visible translational motion as well. In general, this motion is confined to ranges of less than a micron on the time scales we look at, though it can be greater on 


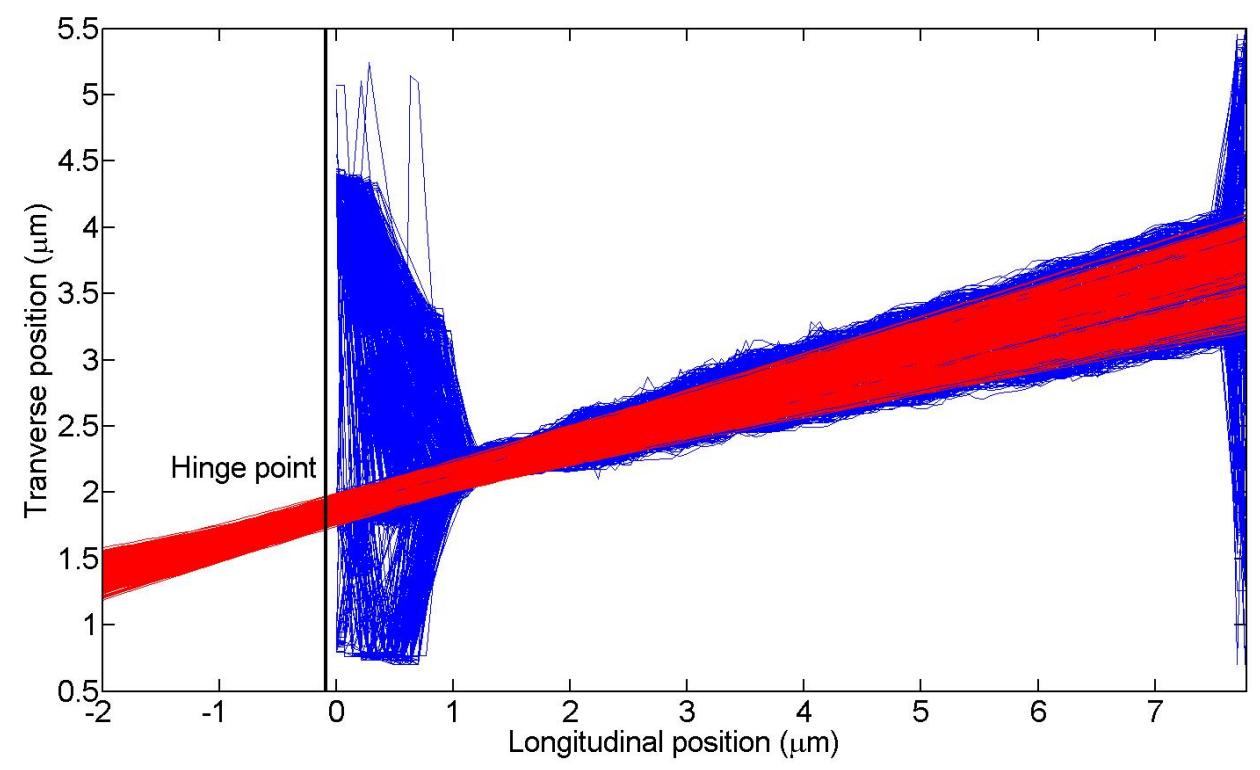

Figure 6.28: Hinge point analysis. A superimposition of backbones is pictured in blue while linear fits to 25-backbone averages is overlaid in red. The calculated hinge point is designated with a solid black line.

time scales of tens of minutes. Translational motion of the base can lead to both an overestimate and an underestimate of the depth of the cilium hinge point and is why we record some positive values (i.e. above the cell membrane) for the hinge point position.

\section{Active fluctuations}

Since the cilium is embedded in an active medium (the cell) and its hinge point appears to be inside the cell, we checked to see if the cilium fluctuations had an active component. To do this, we compared the fluctuations of unperturbed control cells, cells starved of ATP, and cells treated with blebbistatin, an inhibitor of myosin II motor activity (protocols for these treatments can be found in Appendix A). Starving cells of ATP, the main energy source for most cellular motion, is a standard way to determine whether cellular processes are actively driven $[82,83]$. Since the cilium grows from the mother centriole, which is embedded in the actin cortex during ciliogenesis [84], we deemed this a possible candidate for the hinge point. As the centriole is embedded in an actin network, we thought it possible that myosin II motor activity, which generates force in actin networks, could play a role in active fluctuations as it does in in vitro networks [26, 28].

The results of these experiments are shown in Figure 6.29, which plots the angular 


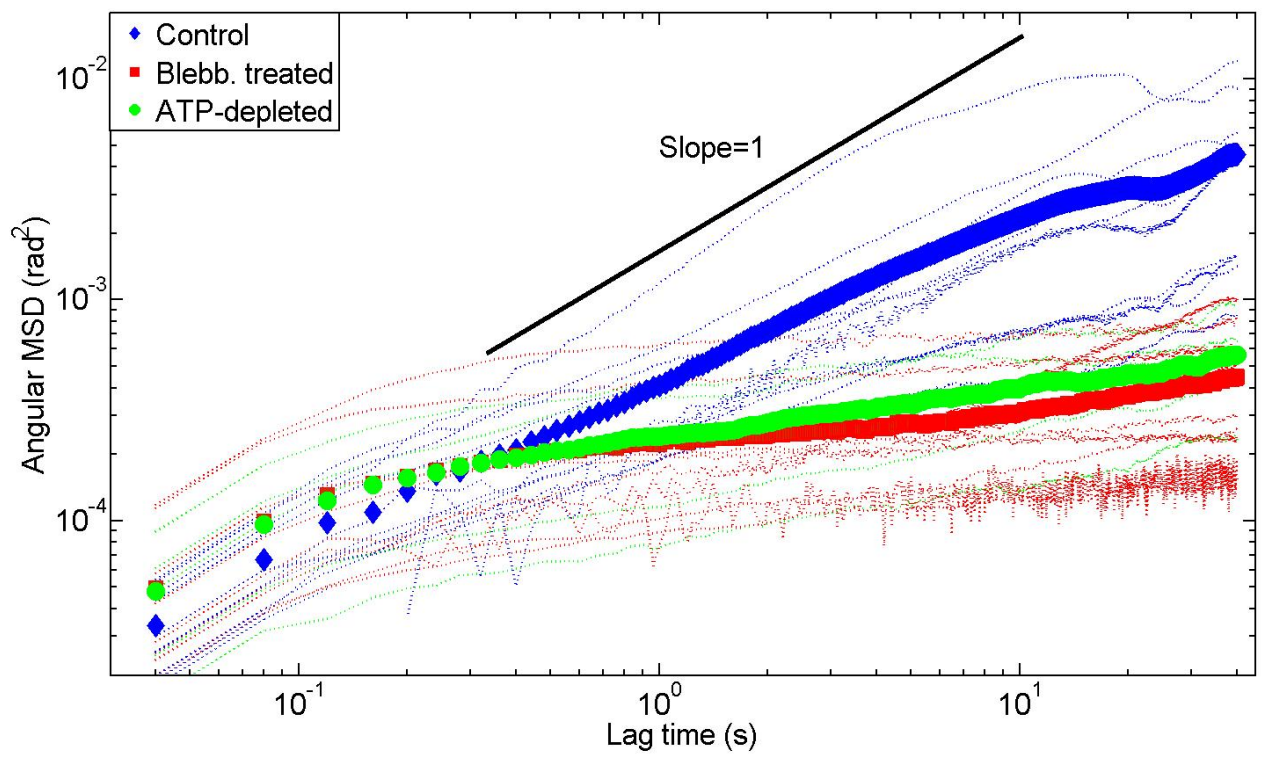

Figure 6.29: Plot of angular mean squared displacement (MSD) vs. lag time. The colors correspond to control (blue), blebbistatin treated (red), and ATP-depleted (green) cells $(\mathrm{N}=10, \mathrm{~N}=9$, and $\mathrm{N}=5$ cilia, respectively, from 6 different sample preparations). The large, filled symbols represent the average MSD for the various conditions while the thin lines trace out the MSDs of the individual cilia. There is a dramatic drop off in the fluctuations of both the ATP-depleted and blebbistatin treated cells, as well as a change in their slopes compared to the control cells (the thick black line shows a slope $=1$ for reference). To correct for the varying noise levels between the different experiments, the intercept of the line going through the first two points of each curve with the ordinate axis was calculated and subtracted from the curve.

mean squared displacement (MSD) vs. lag time for the various conditions. There is a dramatic drop-off in the fluctuation activity of the biochemically perturbed cells in comparison with the control cells, indicating a strong active component to the cilium fluctuations. Additionally, it appears that the entire active component of motion comes from myosin II motor activity, evidenced by the fact that the ATP-depleted and blebbistatin treated curves lie very close to each other, with the blebbistatin curve lying slightly below the ATP-depleted one.

Figure 6.30 shows histograms of the mean angle distributions of the control and blebbistatin treated cells included in Fig. 6.29. The control cells have a much wider distribution of angles than the blebbistatin treated ones and make larger angular excursions $\left(\sigma_{\text {control }} \approx 0.06\right.$ radians vs. $\sigma_{\text {blebb }} \approx 0.02$ radians $)$.

There are also changes in the MSD scaling behavior between the control and 


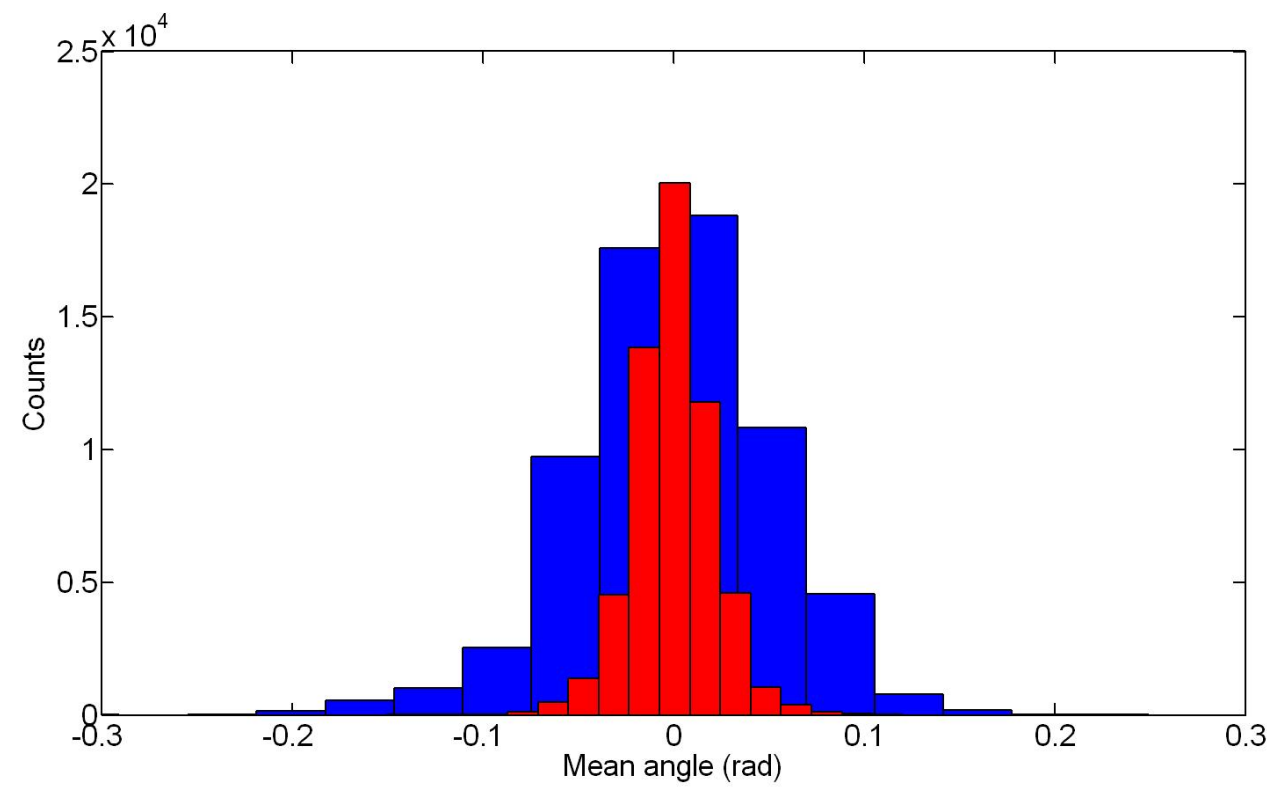

Figure 6.30: Histograms of mean angle distributions from Fig. 6.29. The distribution of control cell mean angles is colored blue and the distribution of blebbistatin treated cell mean angles is colored red. The ATP-depleted distribution essentially overlays the blebbistatin-treated distribution and is omitted for clarity of presentation.

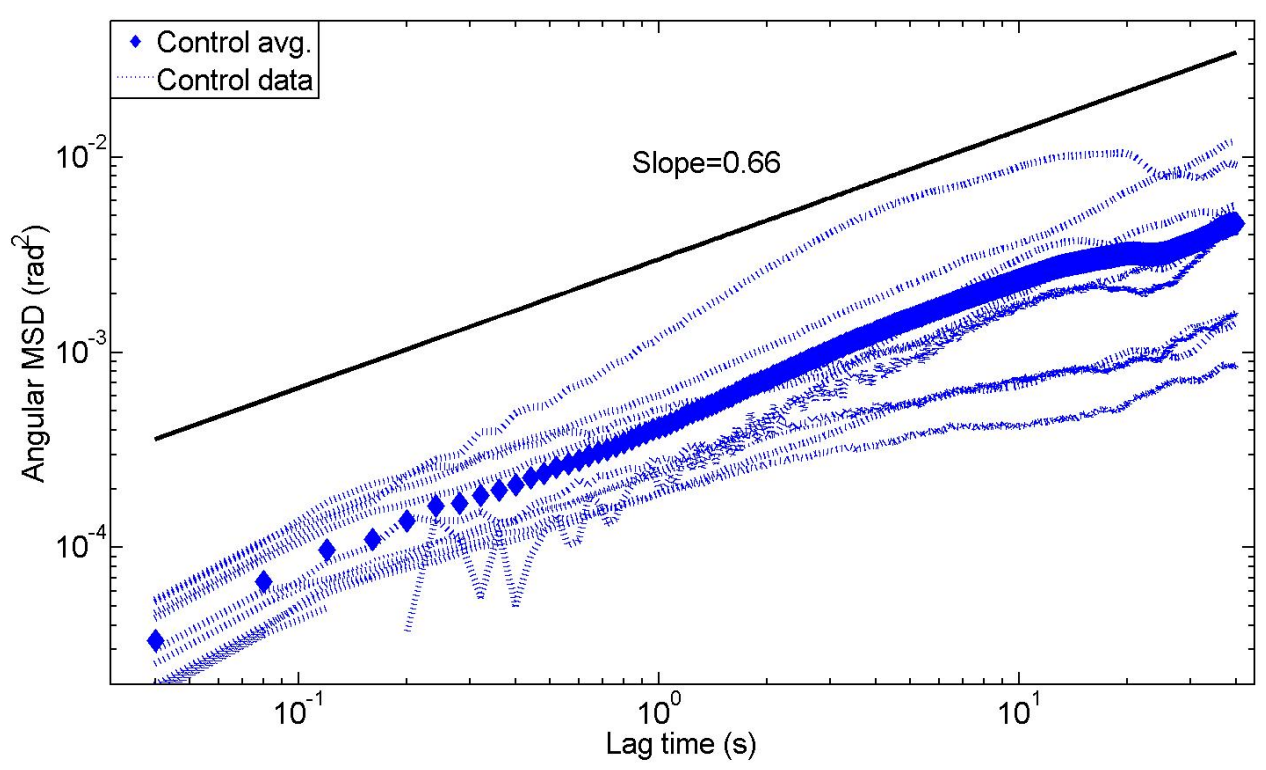

Figure 6.31: Detail of control cells from Figure 6.31. The thick black line shows the a fit to the averaged MSD scaling behavior with a slope of 0.66 . 


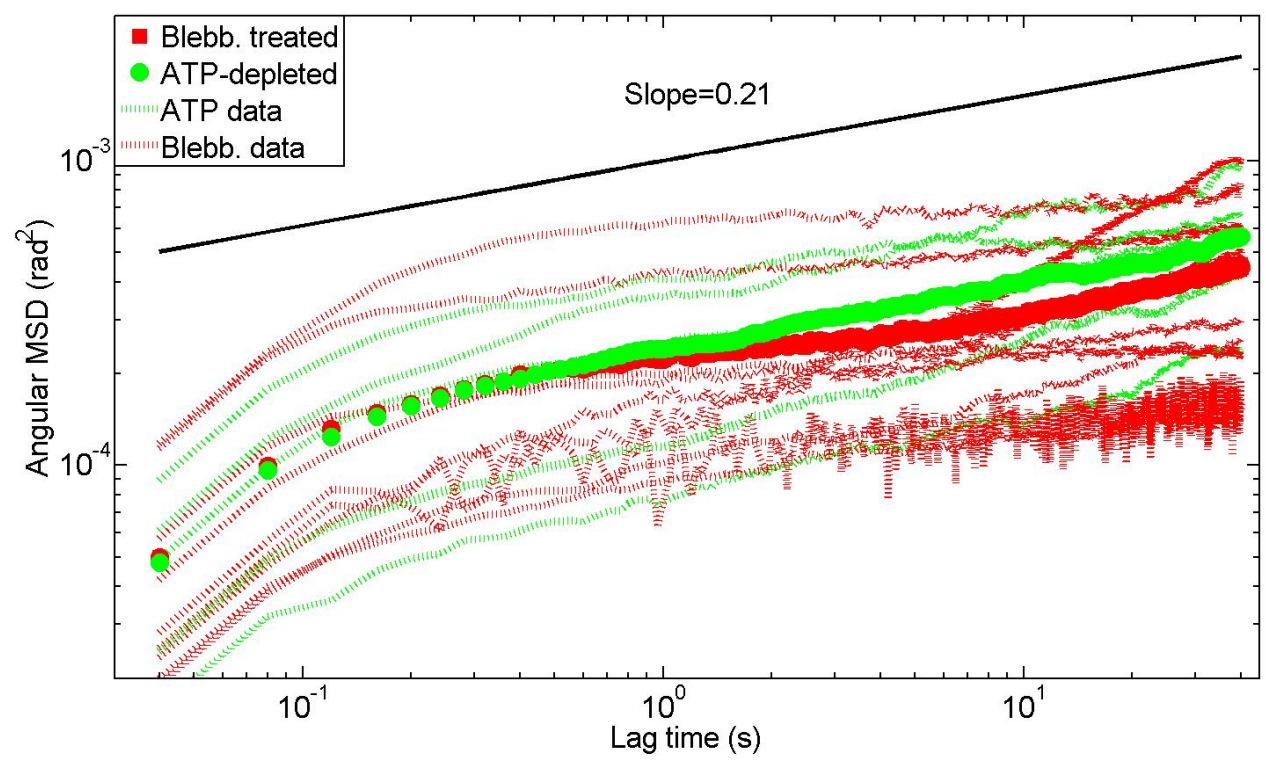

Figure 6.32: Detail of ATP/blebbistatin cells from Figure 6.31. The thick black line shows a fit to the averaged MSD scaling behavior with a slope of 0.21 .

perturbed cells. Figure 6.31 shows just the control cells from Figure 6.29 along with a fit to the scaling behavior of the averaged MSD. On average the control group exhibits a sub-diffusive scaling exponent of 0.66 in the angular MSD, though there is some variety in the individual scaling of the cilia in this group. This stands in contrast to Figure 6.32, which shows the scaling behavior of the ATP-depleted and blebbistatin treated groups. Both of these groups exhibit an MSD scaling exponent with a slope of 0.21 , significantly lower than that of the control cells, indicating that these cilia fluctuate "more sub-diffusively".

It is somewhat curious that the averaged ATP-depleted curve lies above that of the blebbistatin treated one in Figure 6.31. Since myosin II motor activity is dependent on ATP, one would expect the ATP-depleted curve to lie at or below the blebbistatin treated curve, not vice versa. Interestingly, the "most active" ATPdepleted cilium curve, i.e. with the largest angular MSD, is from a sample preparation in which reorganization of the cell monolayer, evidenced by a shrinking in height [83], occurred during the fluctuation measurement. If we consider this cilium to be an "outlier" and re-average the remaining four ATP-depleted curves, we obtain the black curve shown in Figure 6.33. Here the re-averaged, ATP-depleted MSD curve coincides with the blebbistatin treated MSD curve, as one would expect. We emphasize that this cilium cannot be excluded due to traditional outlier rejection criteria, e.g. lying more than 2-3 standard deviations away from the mean, and 


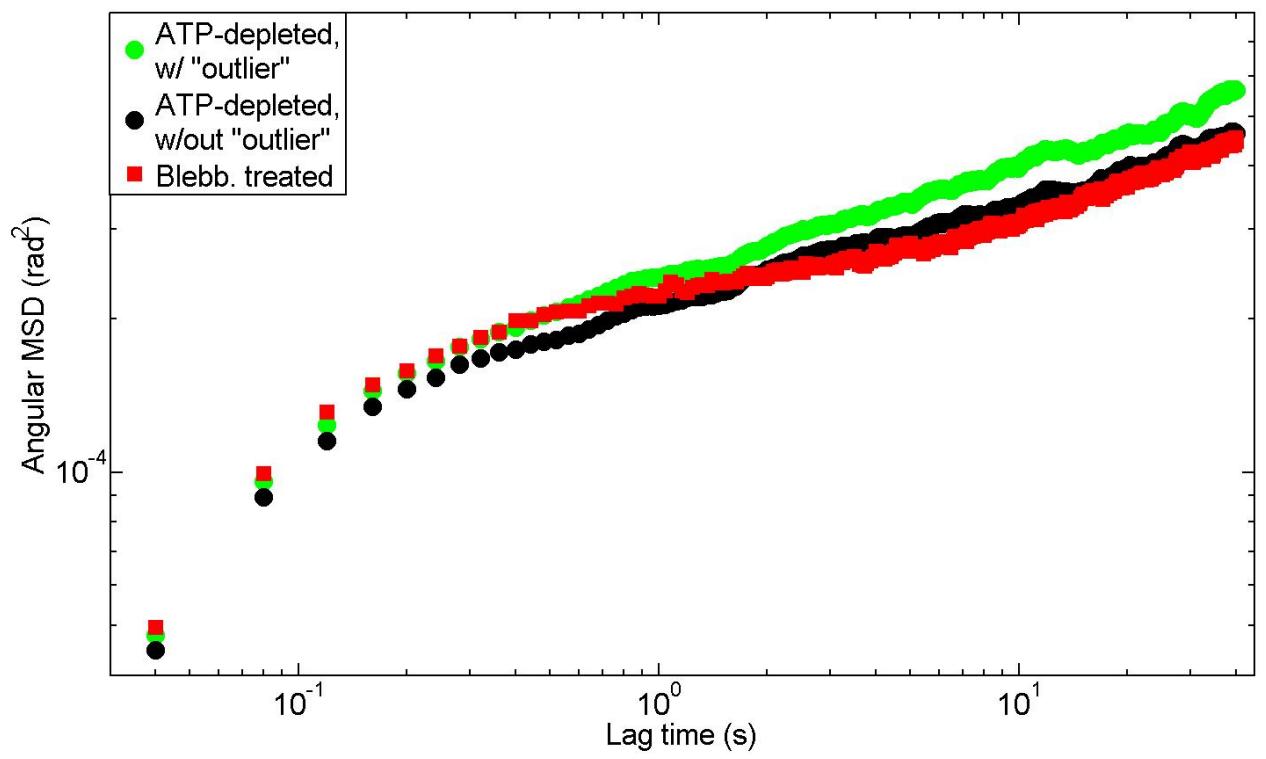

Figure 6.33: Comparison of average angular MSDs with/without "outlier". The curves show the average MSDs for the blebbistatin treated cells (red), the ATPdepleted cells (green), and the ATP-depleted cells excluding the "outlier" cilium described in the text (black).

this is presented only as one possible explanation for the relative positions of the ATP-depleted and blebbistatin treated MSD curves. The discrepancy could also be due simply to statistical fluctuations combined with small sample sizes.

\section{Errors}

We have already described the performance of our tracking algorithm in Chapter 5, so here we mention the main source of noise in the videos themselves, which again comes from debris floating in the extracellular solution. When this debris diffuses through the imaging field-of-view (FOV), the backbone tracking often tracks the debris spuriously, leading to errors. Dirt on the camera/optics, in the form of dark, unmoving specks in the FOV, also disrupts cilium tracking. When the cilium moves across such a speck, the large intensity gradient between the black speck and the background leads to a large edge signal and the tracking algorithm mistakenly tracks the speck position. This is the source of the oscillations in variance vs. the longitudinal position in some of the transverse scaling plots.

Flows also present a potential problem in the analysis of cilium fluctuations, especially in the differentiation between active and passive motion. To avoid flow in our sample chamber, we sealed the chamber entirely, as described in the Experimen- 


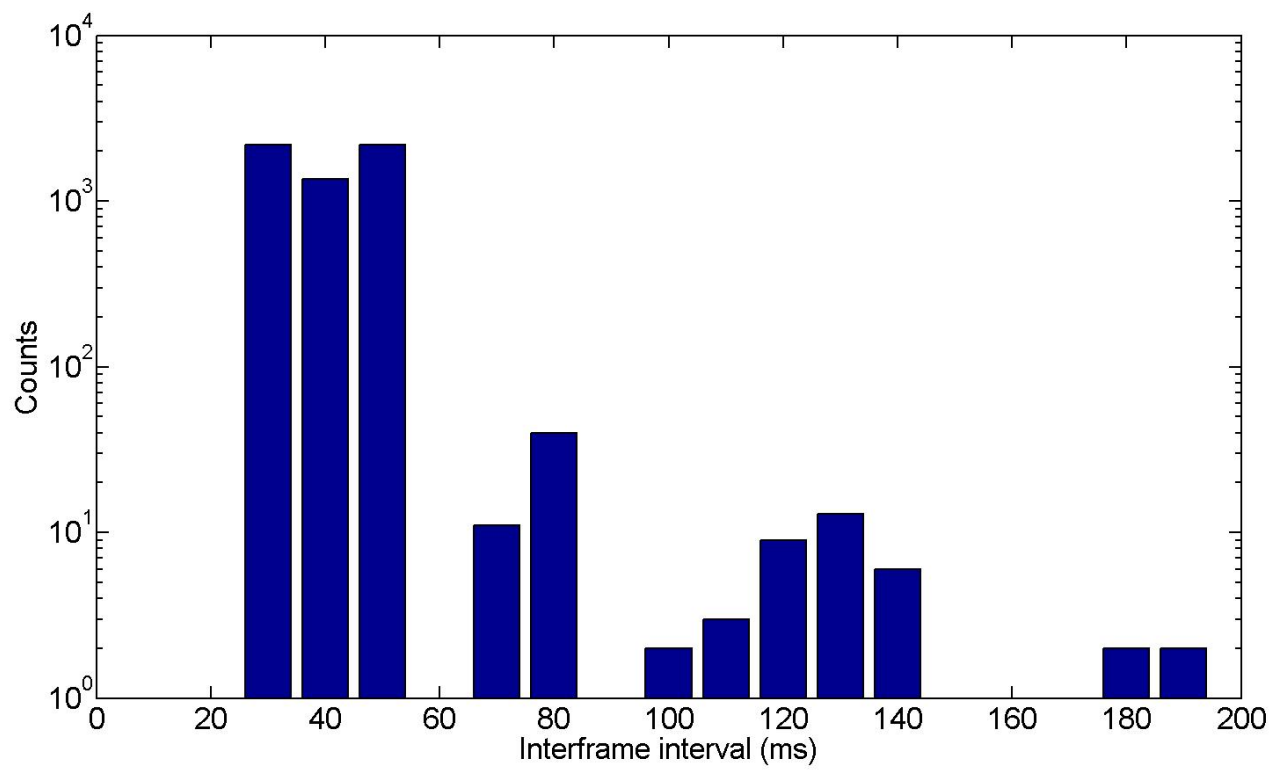

Figure 6.34: Histogram of interframe intervals in a movie. The ordinate is logarithmically scaled, to emphasize the rarer events where the time interval is greater than normal.

tal methods chapter. We also looked for signs of flow by observing the background debris for an overall flux in a particular direction. In addition, we looked for skewness in the histograms of the angular distribution, reasoning that flows would bias the cilium angle in a particular direction and suppress fluctuations in the other. Finally, on the rare occasions when two cilia were both visible in the same frame, we performed a correlation analysis of the motion of the two cilia to determine whether they were moving in a correlated manner, another possible sign of flow. Movies in which evidence of flow was found were discarded and not included in our analysis.

After our data had been collected, we noticed an unexpected source of error related to our camera acquisition rate. Figure 6.34 shows a histogram of time intervals between subsequent frames from one of our movies. While $>98 \%$ of the frame-to-frame time intervals are $40 \pm 10 \mathrm{~ms}$ long, 80 out of 5800 frames have longer frame-to-frame time intervals. This most likely stems from the computer "dropping" frames due to sub-optimal processing power, an issue which we are currently addressing. This view is supported by the fact that the anomalous time differences are approximately equal to multiples of 2,3 , and 4 of $40 \pm 10 \mathrm{~ms}$.

To see what effect this might have on the results presented above, we inserted a number of copies of the previous frame in the time series gaps according to the length of the gap. For example, if there was a gap of $80 \mathrm{~ms}$ between frame $a$ and $a+1$, we assumed one frame had been dropped and we inserted a copy of frame $a$ 


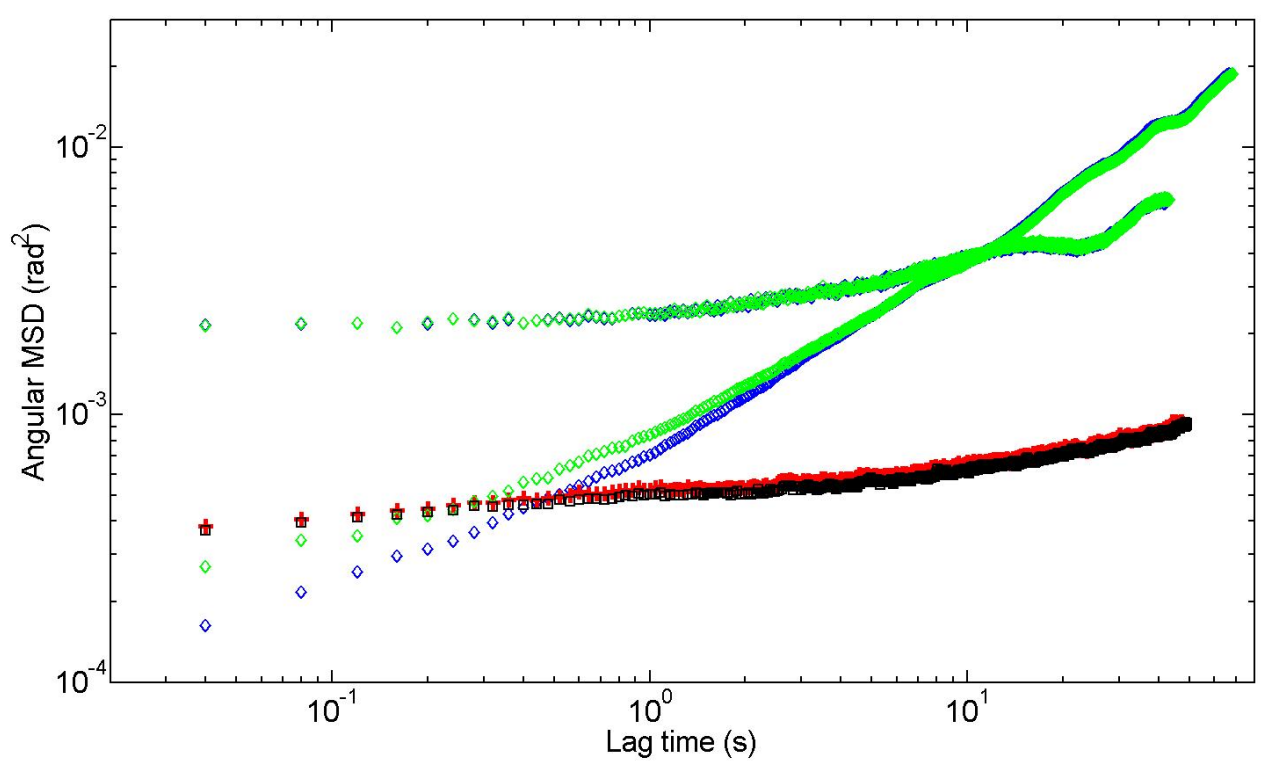

Figure 6.35: Plots of the angular MSD for three cilia with and without gaps in the time series. The blue curves represent the MSDs of control cilia from the experiments above with gaps in the time series and the green curves show the MSDs of the same cilia with added frames. The red and black curves likewise show the MSD for a blebbistatin treated cilium with and without gaps in the time series, respectively.

between the two. So in this case the time series $a, a+1$ became $a, a, a+1$. Likewise if there was a gap of $110 \mathrm{~ms}$ we assumed that two frames had been dropped and two copies of $a$ were inserted, so the time series $a, a+1$ became $a, a, a, a+1$. We chose this method rather than linearly interpolating the cilium backbones between frames since the gaps were larger than a single frame in some instances. After inserting the additional frames we re-ran our tracking algorithm and analysis. A comparison of the angular MSD for three cilia with and without gaps in the time series is shown in Figure 6.35. There is a slight quantitative difference, with the gaps causing a minor overestimate of the MSD at larger lag times and an underestimate in one curve at shorter lag times, but no change in the qualitative behavior of the curves. From this analysis we concluded that the frame dropping doesn't significantly change the results presented above. 


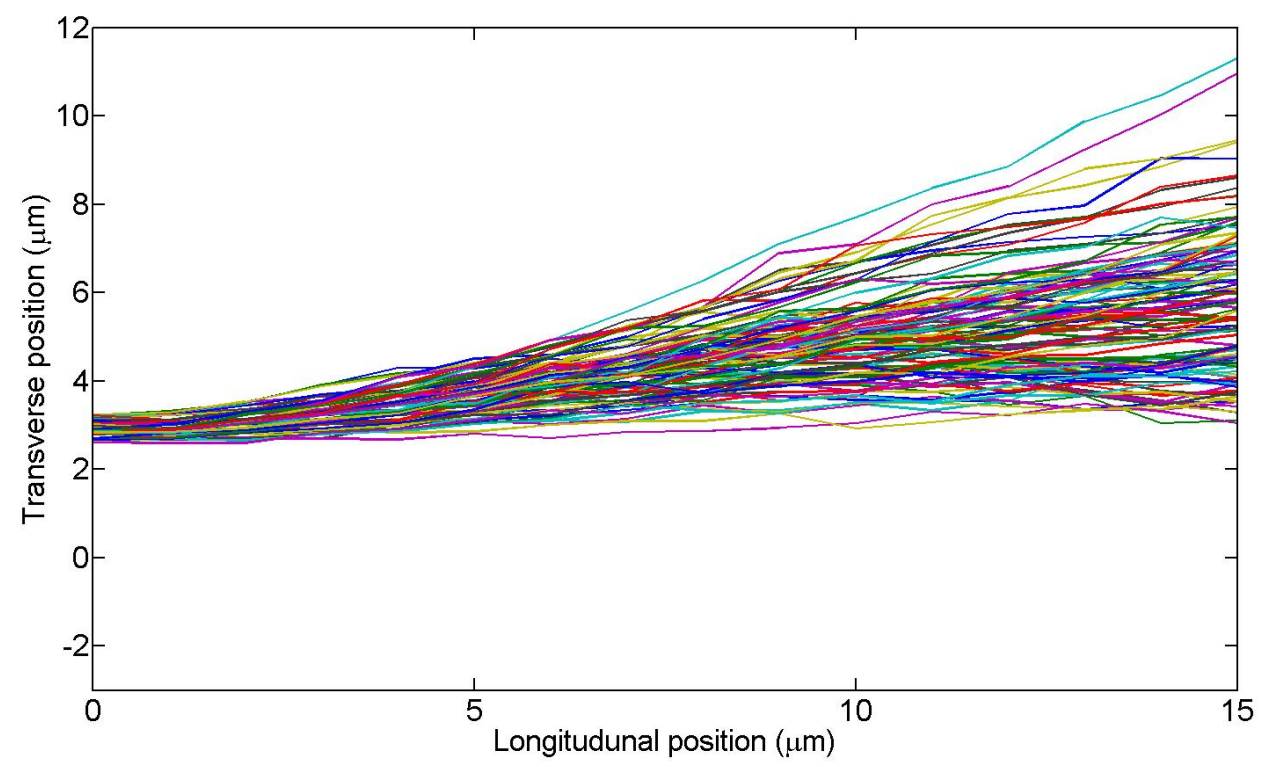

Figure 6.36: Backbone plot reconstructed from confocal stack data. Here the longitudinal position refers to the $z$ coordinate and the transverse position refers to the $x$ coordinate. There is significantly more bending than we observe in our edge-viewing data, as expected from a cilium of this length $(\sim 15 \mu \mathrm{m})$.

\section{$3 \mathrm{D}$ confocal data vs. edge-viewing geometry data}

In this section we present our analysis of data collected by Carolyn Ott (National Institutes of Health, Cell Metabolism and Biology Branch, Lippincott-Schwartz lab). The experimental methods used to collect the data and our tracking/reconstruction of it is described in earlier chapters. Briefly, Carolyn grew the cells on glass and then took confocal stacks of cilia over time, in order to image their dynamics in three dimensions. All of her experiments were performed at physiological temperature $\left(37^{\circ} \mathrm{C}\right)$, unless otherwise noted. In the following the $z$ coordinate refers to the coordinate normal to the apical cell membrane (i.e. generally along the cilium length) whereas $x$ and $y$ are coordinates orthogonal to the $z$ coordinate and each other. Longitudinal will be used to designate the $z$ coordinate, whereas transverse will designate either the $x$ or $y$ coordinate. We reconstructed cilia backbones from this data and tracked their fluctuation dynamics in a manner similar to the preceding experiments performed in an edge-viewing geometry. The distance between $z$ stack slices was $1 \mu \mathrm{m}$ unless otherwise noted.

We first built up backbone plots of the cilium, as shown in Figure 6.36. The cilium pictured in this plot is $50 \%$ longer than the longest cilium in our previous analysis and as such one observes more bending motion, visible in the evident cur- 

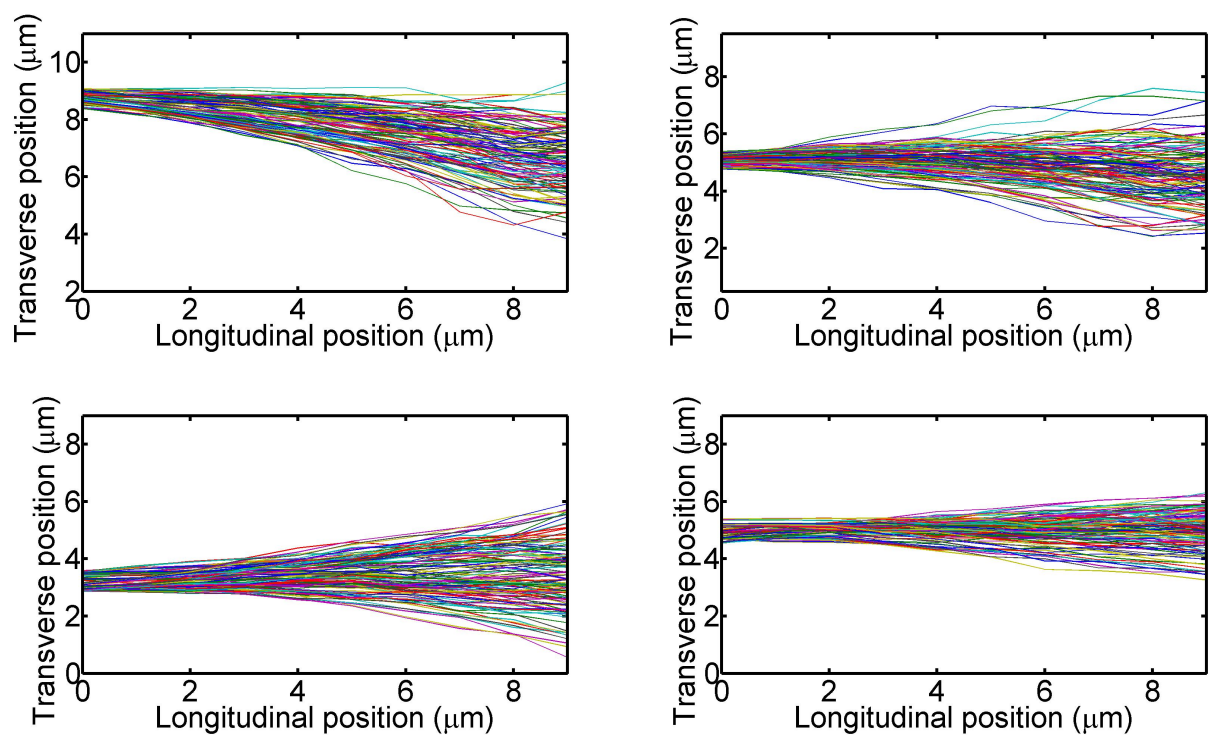

Figure 6.37: Backbone plots from two cilia (top/bottom) taken at $37^{\circ} \mathrm{C}$. The lefthand graphs show $x$ vs. $z$ plots and the right-hand graphs show $y$ vs. $z$.
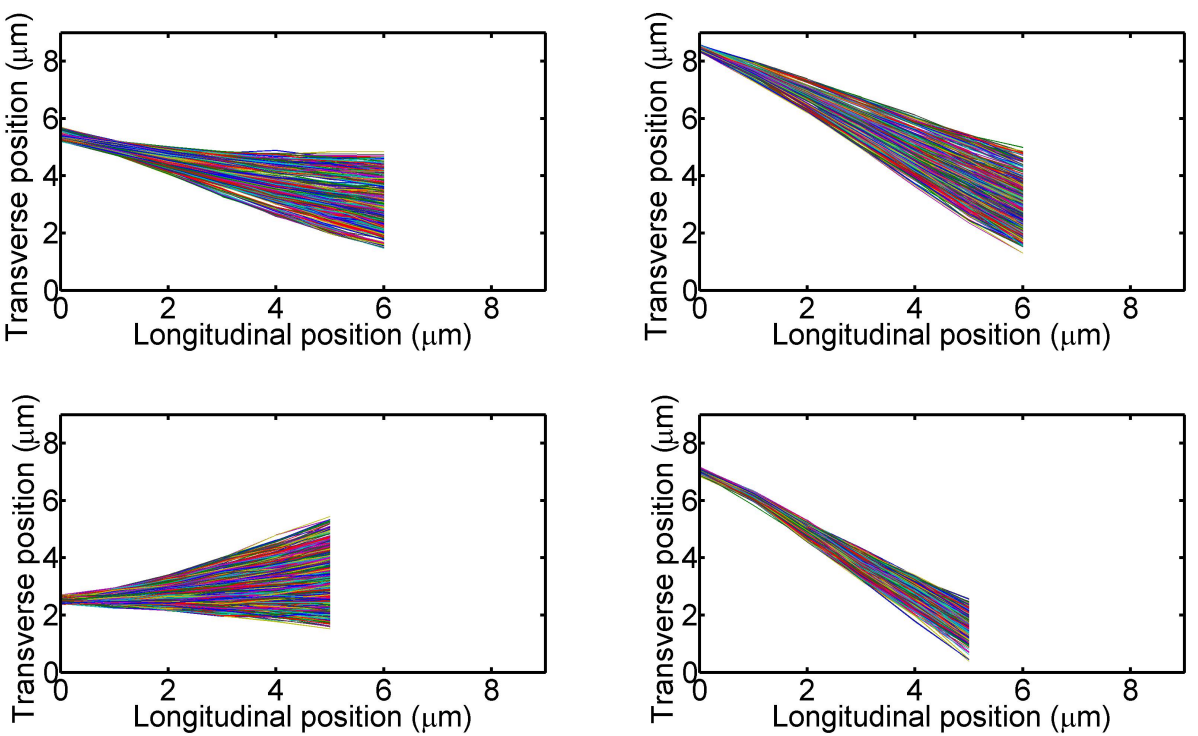

Figure 6.38: Backbone plots from two cilia (top/bottom) taken at room temperature. The left-hand graphs show $x$ vs. $z$ plots and the right-hand graphs show $y$ vs $z$.

vature of some backbones. Figures 6.37-6.39 show $x$ vs. $z$ and $y$ vs. $z$ backbone plots taken from 6 cilia from 4 different experiments. The cilia pictured in Figures $6.38 \& 6.39$ were imaged at room temperature, the rest at $37^{\circ} \mathrm{C}$. 

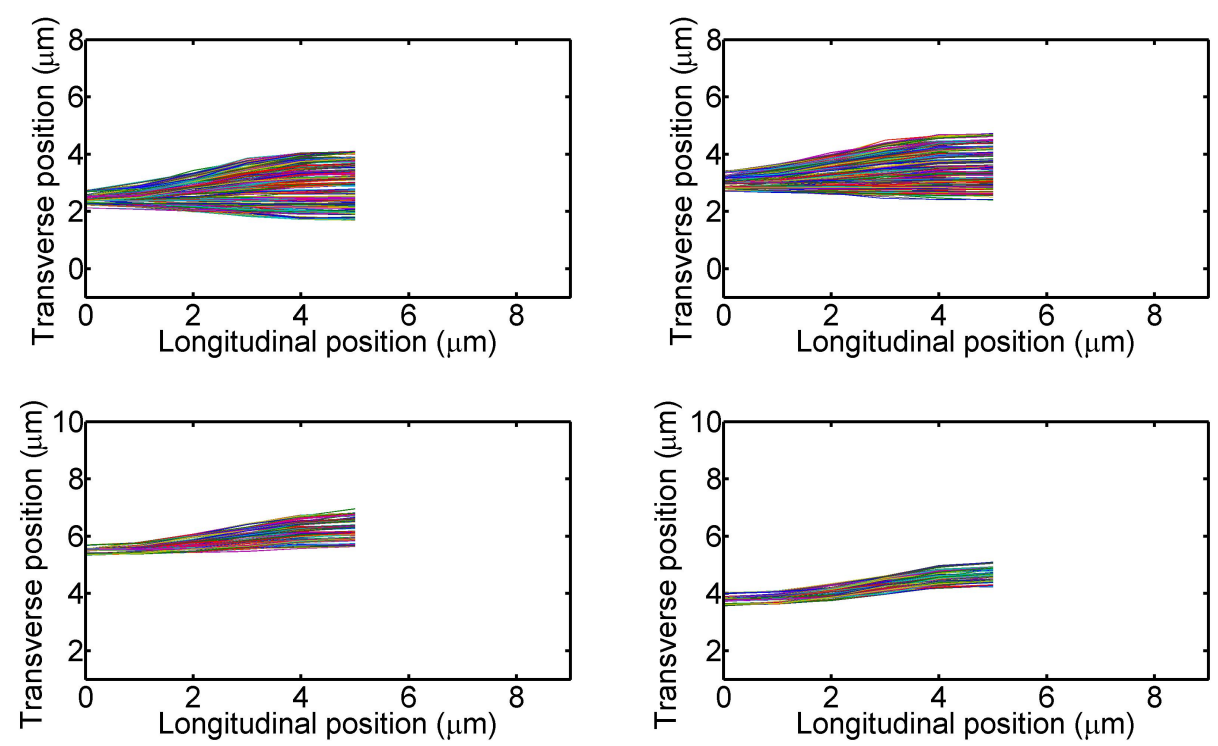

Figure 6.39: Backbone plots from two cilia (top/bottom) taken at room temperature. The left-hand graphs show $x$ vs. $z$ plots and the right-hand graphs show $y$ vs. $z$.

Interestingly, in several plots there appears to be more bending motion than for cilia of similar lengths viewed in the edge-viewing geometry. This greater contribution to cilium motion from bending undermines our previous analysis technique of using the angular fluctuations as a proxy for overall cilium motion. This failure is demonstrated in Figure 6.40, where we reproduce the previously introduced transverse fluctuation, angular collapse, mean angle, and transverse scaling plots for the cilium pictured in Fig. 6.36. The angles in the angular collapse plot (top right panel) fail to convincingly converge to one value over the time series, indicating a large contribution to the cilium motion from bending. The transverse scaling plot (bottom right panel) also shows a deviation from $l^{2}$ scaling (thick black line) for points farther away from the base $(>7 \mu \mathrm{m})$. This is intuitively clear; for a flexible rod on a limited hinge undergoing bending from thermal fluctuations, the contribution of the bending motion relative to hinging motion will increase with increasing length along the rod.

In order to compare the edge-viewing data with the confocal data, we decided to compare the transverse variance as a function of position along the cilium length. This is analogous to the data presented in the transverse scaling plots. We analyzed the variance of all the curves over a time interval of the same length (160 seconds). The transverse variance includes all components of motion (translational, rotational, and bending), and as such is a reasonable measure of overall cilium motion. The dominant type of motion, either rotational or bending, is encoded in the scaling behavior of the transverse variance along the length of the cilium, captured by 

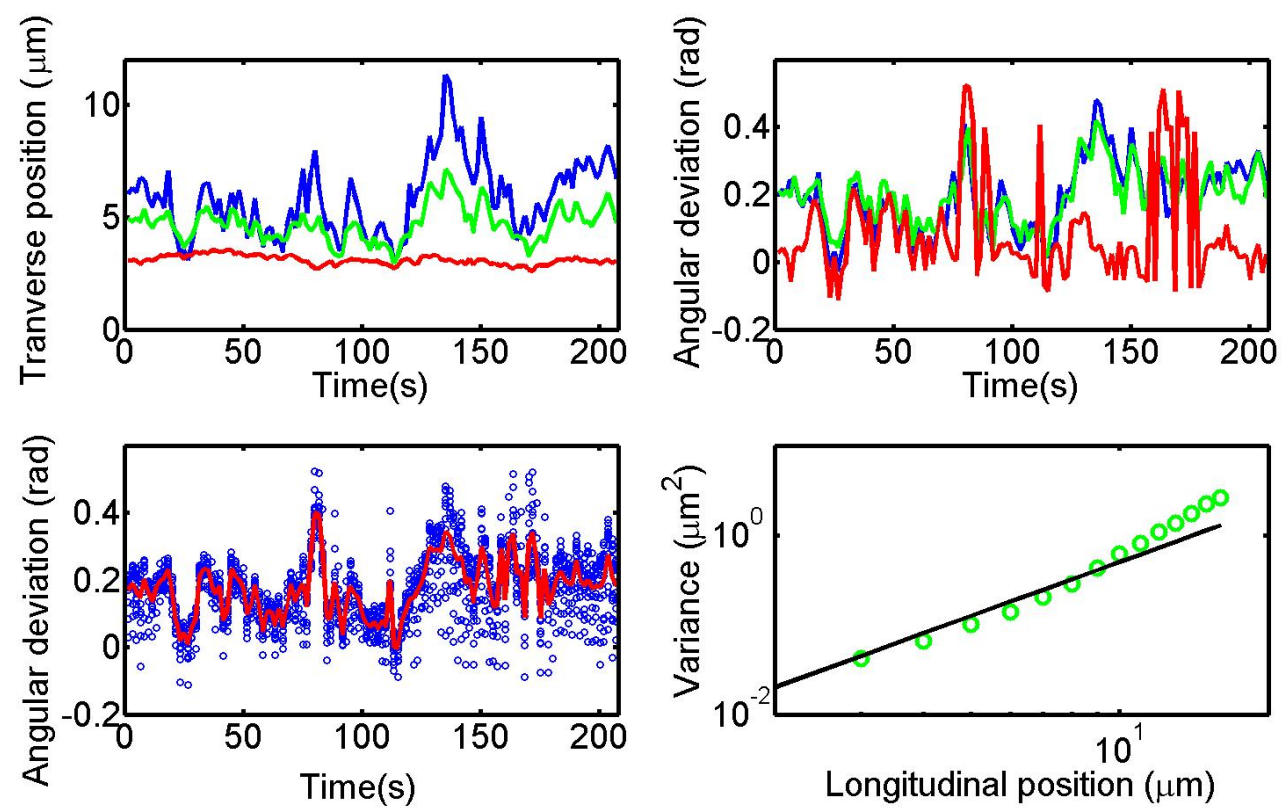

Figure 6.40: Transverse fluctuation, angular collapse, mean angle, and transverse scaling plots for the cilium pictured in Fig. 6.36 ( $x$ vs. $z$ ). The angular collapse and transverse scaling plots (right-hand panels) show a greater contribution to the cilium movement from bending.

the exponent $r$ in the expression $\left\langle d^{2}\right\rangle \propto l^{r}$, as discussed in the previous section. Exponents of $r<2$ indicate convex-inward bending, exponents of $r>2$ indicate convex-outward bending, and exponents of $r=2$ indicate stiff-rod rotational motion. Figure 6.41 shows transverse variance as a function of position along the cilium for both confocal and edge-viewing data. There are jumps in the averaged edgeviewing data curves, particularly above $\sim 5 \mu \mathrm{m}$ in the control cells. The difference in cilia heights and trackable sections of the cilia are the culprits here; fewer cilia are included in the average above $5 \mu \mathrm{m}$, and they cluster around a lower value. The curved "tails" at the beginning of both the edge-viewing curves come from the "average" of a single cilium, the only one trackable at that distance from the base. These factors notwithstanding, the variance between longitudinal positions of $\sim 2.5-5 \mu \mathrm{m}$ and $\sim 6-10 \mu \mathrm{m}$ can be meaningfully compared. The confocal data sits consistently above the edge-viewing data, indicating more overall transverse cilia movement. Some of this is possibly due to the additional contribution to the motion of bending, but it persists even close to the cilium base. The confocal data taken at $37^{\circ} \mathrm{C}$ and at room temperature essentially overlap, suggesting that temperature is not a control variable for overall cilium motion. The variance of the blebbistatin treated cilia lies far below the other curves, as expected. 


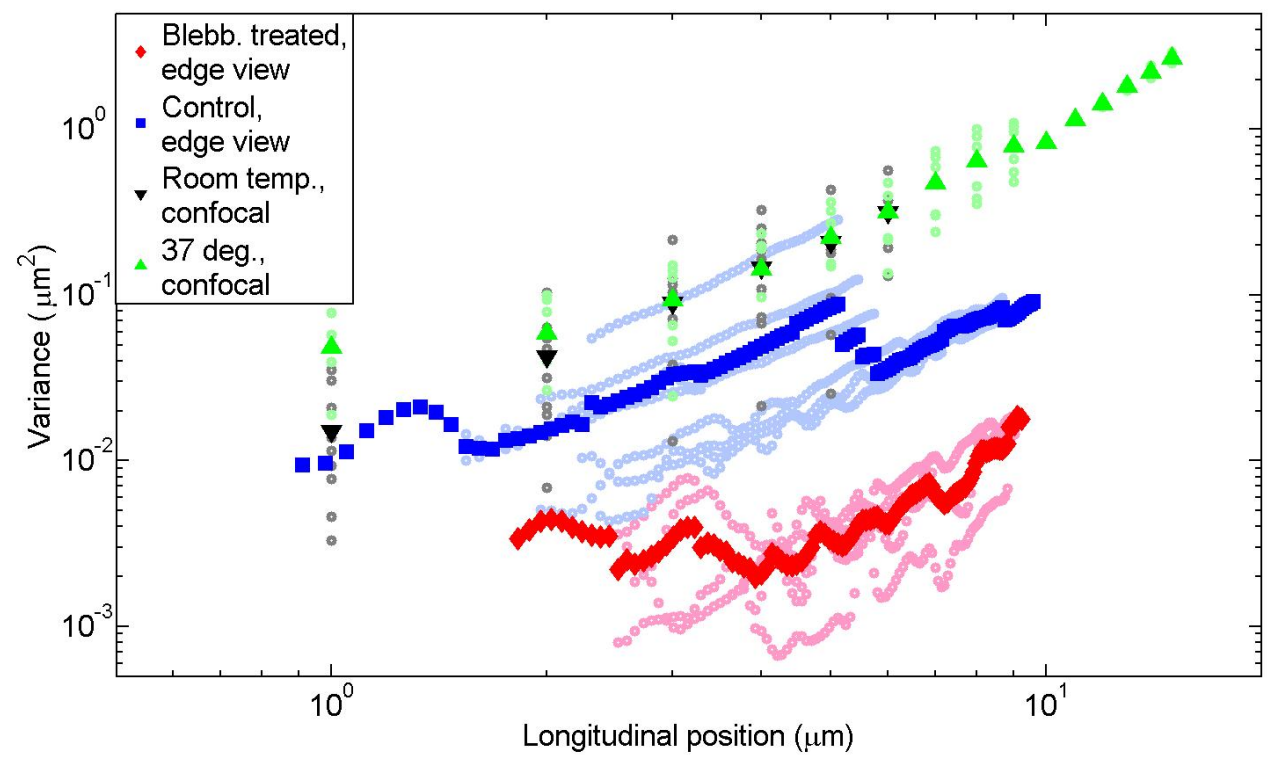

Figure 6.41: Transverse variance vs. longitudinal position. Cilia from confocal stacks taken at room temperature are shown in black, cilia from confocal stacks taken at $37^{\circ} \mathrm{C}$ are shown in green, control cilia from the previously described edge-viewing experiments are shown in blue, and blebbistatin treated cilia from the edge-viewing experiments are shown in red. The heavy, filled symbols in the foreground are averages of the associated data while the individual curves are pictured in lighter colors in the background. The $x$ and $y$ data from the confocal stacks are considered to be independent and are included in the average as separate measurements.

The scaling behavior of the variance curves contains information about the dominant type of motion present. The average scaling exponents for the green, black, blue, and red curves in Fig. 6.41 are, respectively: $r_{37}=2.69 \pm 0.51, r_{R T}=$ $2.23 \pm 0.53, r_{\text {cont }}=1.85 \pm 0.34$, and $r_{b l e b b}=2.04 \pm 0.47$. These exponents emphasize what was already apparent from the backbone analysis, namely that there is a greater bending contribution to cilium motion in the confocal data. It is interesting that there appears to be more bending in the cilia analyzed at physiological temperature, but not too much should be read into this. This sample has systematically longer cilia, which exhibit more bending generically, so better statistics from samples with comparable cilia lengths would be necessary to determine whether there is a temperature contribution to bending. Finally, a note on fitting is in order. These scaling exponents indicate the mean of all the individual scaling exponents, fit over the entire (or close to entire) cilium length. If one fits just the second half of the curves, containing points closer to the cilium tip, one finds scaling exponents of 

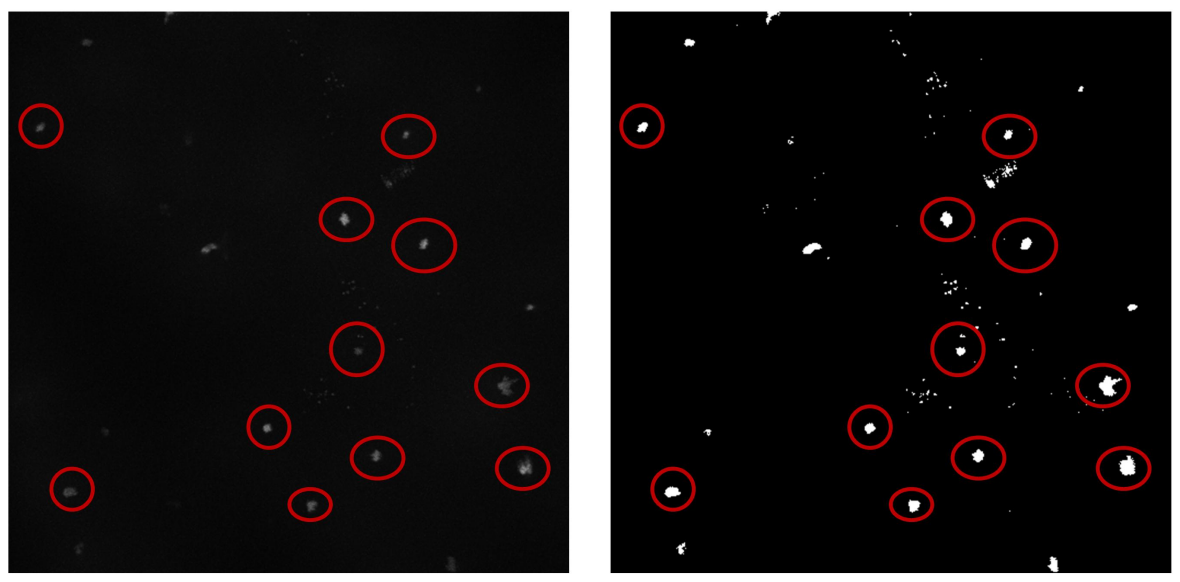

Figure 6.42: Maximum intensity projection from a single confocal slice. The lefthand panel shows the maximum intensity projection while the right-hand panel shows the thresholded image used to find the best-fit ellipse. The red circles designate cilia which were analyzed.

$r_{37} \approx 3$ and $r_{\text {cont }} \approx 2$ for the $37^{\circ} \mathrm{C}$ and control data (the other two exponents stay roughly the same), suggesting nearly pure bending and rotational motion near the tip in these respective groups of cilia.

\section{Fluctuation anisotropy}

The cilium fluctuations that we see in our edge-viewing geometry are actively driven by myosin II activity, and thus potentially by actin-myosin contractility in the actin cortex. The cilium anchorage in the cortex is via the centrosome which consists of mother and daughter centrioles [84, 85]. The cilium grows out of the mother centriole which is in turn connected to the daughter centriole, which lies at a right angle to the mother centriole, forming an "L" [85, 2]. Given this symmetry breaking at the cilium anchorage point, we examined the confocal fluctuation data for signs of anisotropy. To do this we created a maximum intensity projection with ImageJ of one confocal slice at a height of $3-4.5 \mu \mathrm{m}$ (the slice height varied between movies). Heights in this range were chosen from the expectation that any anisotropy in the fluctuations due to asymmetries in the base embedment would decay with increasing height along the cilium due to the onset of thermal bending. The maximum intensity projection highlights the shapes traced out by the orbits of fluorescent cilia over the course of the movie, as shown in the left-hand panel of Figure 6.42. The right-hand panel shows a thresholded version of the same image, produced with ImageJ using the IsoData thresholding algorithm [86]. The threshold level was adjusted by hand for each cilium until no surrounding noise pixels were contiguous with the orbit area. 


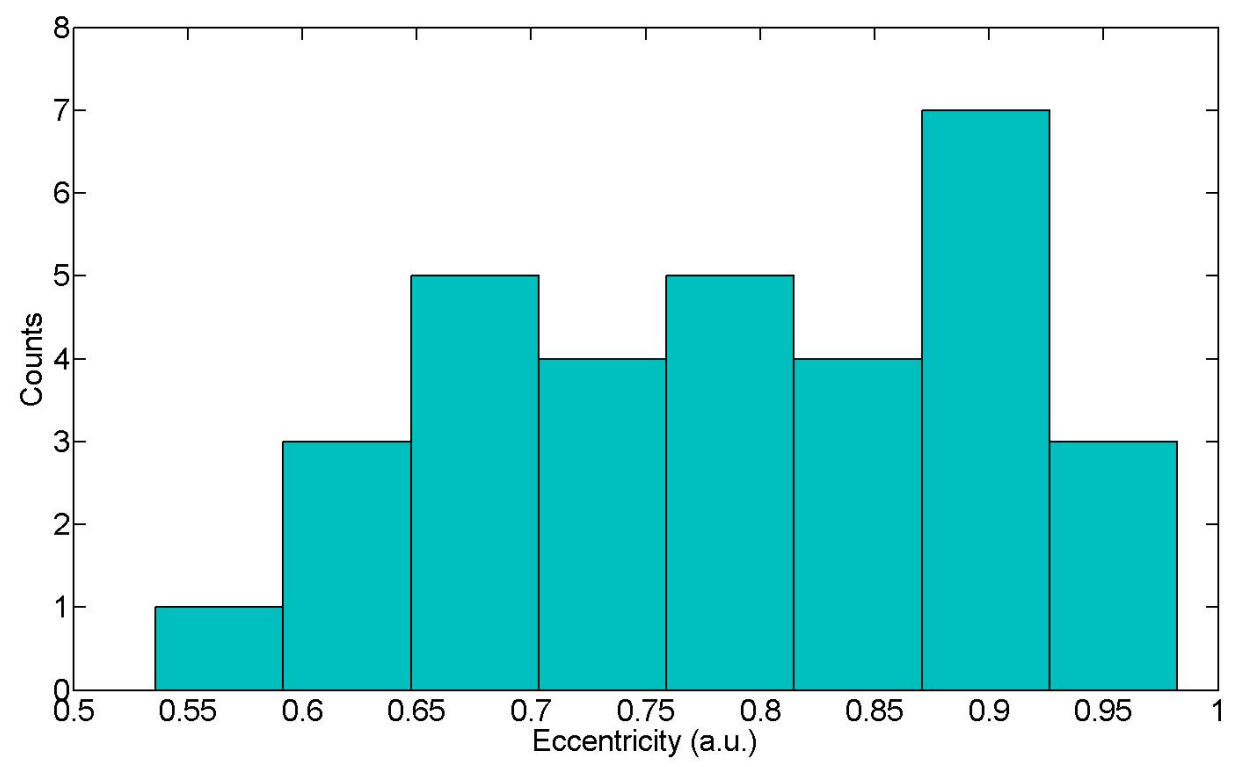

Figure 6.43: Histogram of eccentricities from 32 cilia. The mean eccentricity value is $\epsilon=0.79 \pm 0.12$.

From the thresholded image we then, still using ImageJ, found the best-fit ellipse (region-based method [87]) for each cilium orbit and calculated its eccentricity as a measure of the orbit's anisotropy.

Figure 6.43 shows a histogram of the eccentricities of 32 cilia orbits. We find an average eccentricity of $\epsilon=0.79 \pm 0.12$ for the cilia orbits studied here. Eccentricity is a biased indicator, in that any measurement noise leads to $\epsilon<1$, with $\epsilon=1$ being the eccentricity expected for an isotropically fluctuating cilium orbit. As an informal rule of thumb from cell aspect ratio measurements, $\epsilon>0.83$ can be regarded as circular, i.e. isotropic [88]. As our value lies very close to this, we conclude that, overall, the cilia orbits which we observe are isotropic or very close to isotropic. A possible future step in this direction, to rule out anisotropy definitively, would be to design a simulation to generate isotropic, synthetic data and run the above analysis to ascertain better the effect of noise on our measured $\epsilon$ values.

\section{Cilium-cilium correlations}

From the larger FOVs in the confocal data and the top-down perspective, it was often possible to visualize several cilia in one FOV, opening up the possibility of performing cross correlation analysis on the fluctuations of pairs of cilia. The reason for such analysis was two-fold: one, to check if there were any coupled dynamics between cilia, as in the case of motile primary cilia [89], and two, as a check for 

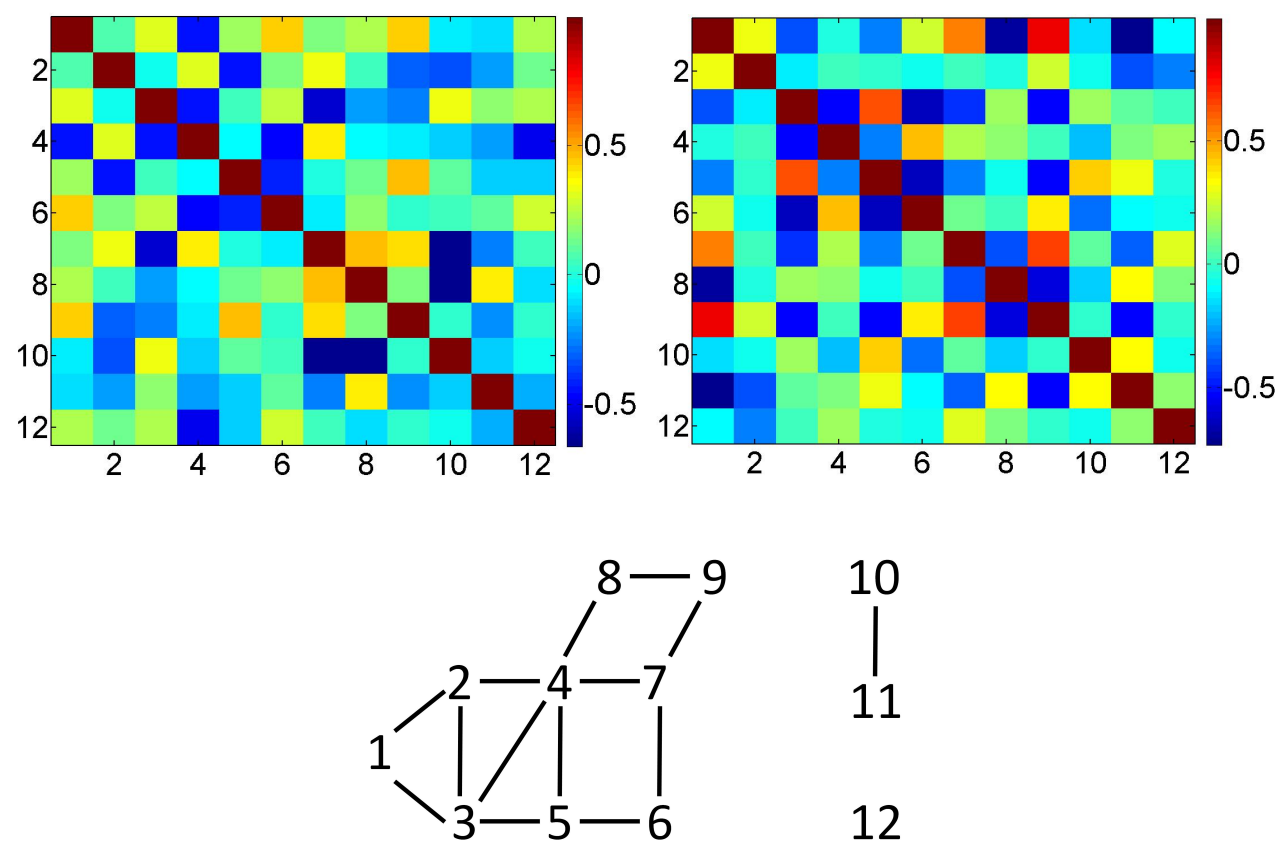

Figure 6.44: Cross correlation matrices (CCMs). The CCM for the $x$ coordinates is pictured in the upper left-hand graph and the CCM for the $y$ coordinates is pictured in the upper right-hand graph. Twelve cilia pairs were analyzed. The color bars to the right of each graph indicate the scale. Underneath the correlation matrices is a connectivity diagram showing which cilia extend from nearest-neighbor cells. Neighboring cells are designated by a line connecting them.

flows in the sample, which would affect our fluctuation measurements. To perform the cross correlation analysis, we tracked the movements of 12 cilia at a height of $4.75 \mu \mathrm{m}$ from the cell membrane for $\sim 90$ seconds at $\sim 3 \mathrm{~Hz}$. We then calculated the Pearson correlation coefficient (PCC), $r$, defined as [90]

$$
r=\frac{1}{n-1} \sum_{i=1}^{n}\left(\frac{u_{i}-\bar{u}}{\sigma_{u}}\right)\left(\frac{v_{i}-\bar{v}}{\sigma_{v}}\right)
$$

where $u_{i}, v_{i}$ represent the $x$ coordinates ( $y$ coordinates) of the two cilia being analyzed at frame number $i$, the $\bar{u}, \bar{v}$ variables stand for the means of the $x$ coordinate ( $y$ coordinate) distributions of the two cilia, and $\sigma_{u}, \sigma_{v}$ are the standard deviations of the $x$ coordinate ( $y$ coordinate) distributions of the two cilia. Here a correlation coefficient of $r=0$ indicates no correlation, $r=1$ indicates perfect positive correlation, and $r=-1$ indicates perfect negative (anti-) correlation. We only perform $x-x$ and $y-y$ cross correlation analysis, never $x-y$. Figure 6.44 shows cross correlation matrices containing the PCC value for each cilia pair, with the $x-x$ 

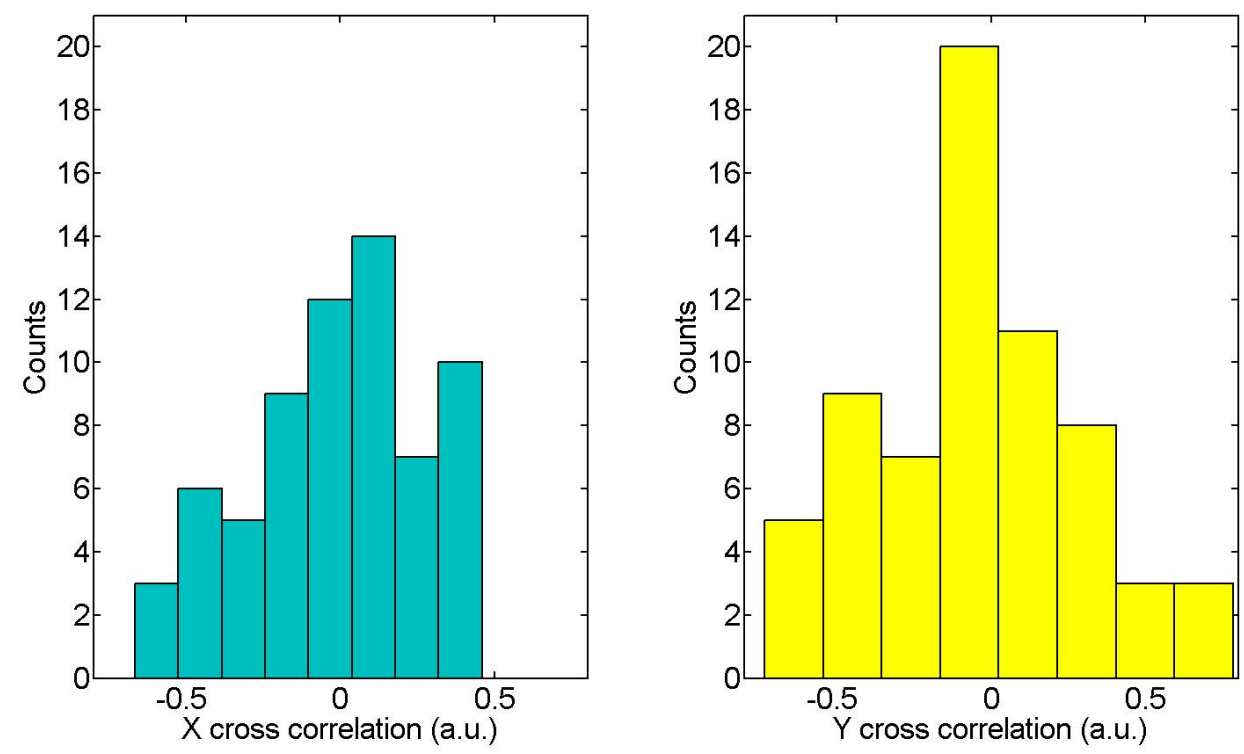

Figure 6.45: Histograms of the $x$ (left-hand) and $y$ (right-hand) PCC values from Fig. 6.44. The mean PCC values for $x$ and $y$ correlations are $r_{x}=-0.01 \pm 0.29$ and $r_{y}=-0.05 \pm 0.34$, respectively.

cross correlation shown in the top left-hand matrix and the $y-y$ cross correlation shown in the top right-hand matrix. Beneath the matrices a connectivity diagram showing which cilia are neighbors (designated by a line connecting them) is included for reference.

The cross correlation matrices exhibit no noticeable correlation structure, and the PCC values are similar to those generated by simulating and cross correlating 12 random walks (data not shown). Figure 6.45 shows histograms of the $x$ and $y$ PCC values, which yield average values of $r_{x}=-0.01 \pm 0.29$ and $r_{y}=-0.05 \pm 0.34$, respectively. Other confocal data subjected to the same analysis yields the same results, leading us to conclude that the cilia fluctuations we track are not moving in a correlated fashion, due to flow or otherwise. 


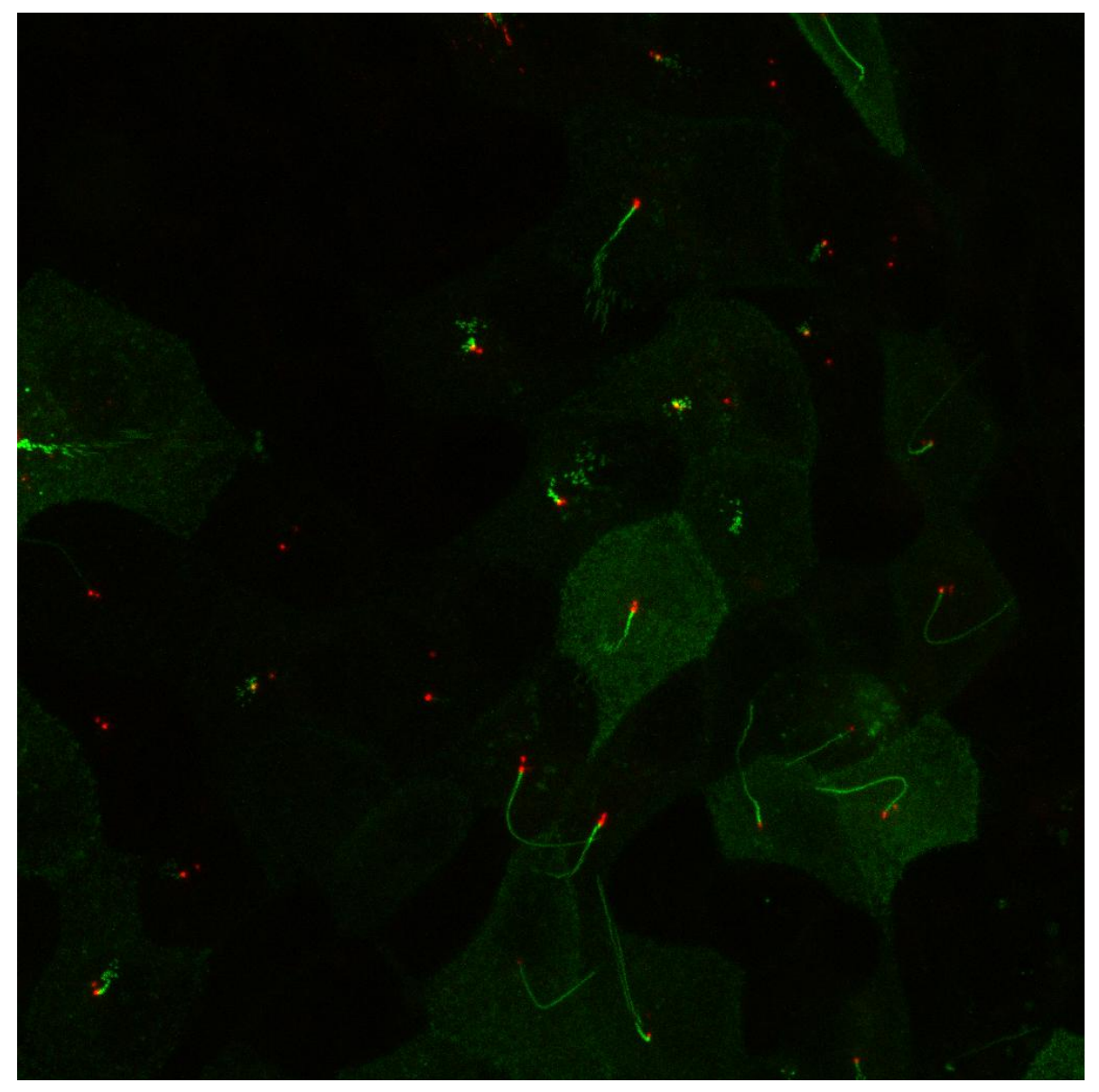

Figure 6.46: Two color overlay of $z$ stack max projections. Fluorescently labeled cilia are pictured in green and fluorescently labeled centrosomes are pictured in red. Picture courtesy of Carolyn Ott.

\section{Cilium-centrosome correlations}

In an effort to confirm our hypothesis that active motion is being generated in the actin cortex and transmitted to the cilium, Carolyn labeled the centrosome complex in her fluorescent cilia line, as described in the Experimental methods chapter. Figure 6.46 shows an overlay of cilia and centrosomes labeled with the two different fluorophores (emerald-MCHR1 and mRFP-PACT), displayed in green and red respectively. She then took confocal stacks over time with two color channels, in order to track both the cilium and centrosome dynamics simultaneously. The movies were taken at a rate of $2 \mathrm{~Hz}$ and data collection was as previously described. To perform our correlation analysis, we tracked a point on the cilium close to the base, $\sim 1 \mu \mathrm{m}$ above the cell membrane and compared these movements to those of the centrosome complex. The five cilium-centrosome pairs analyzed came from 4 


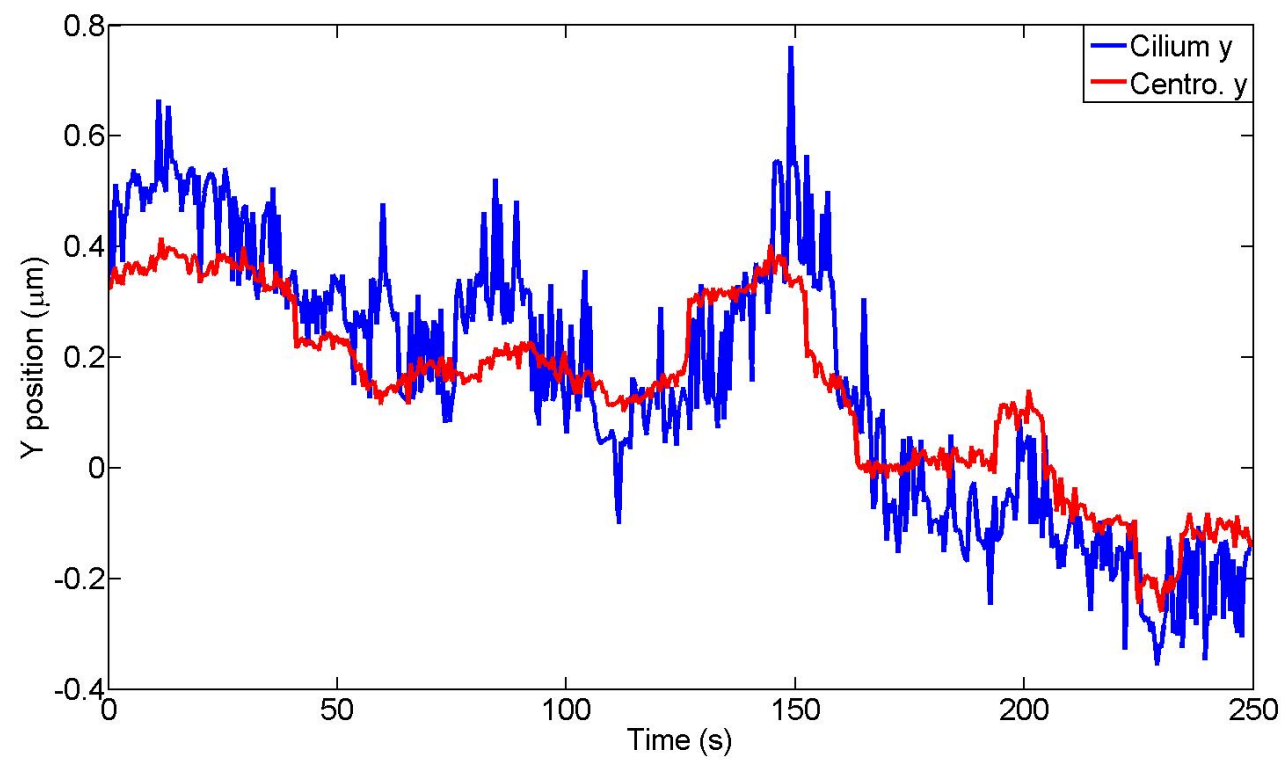

Figure 6.47: Plot of cilium and centrosome $y$ position vs. time. The blue traces tracks the cilium position while the red trace tracks the centrosome position. The two curves exhibit positively-correlated behavior over most of the time course.

different experiments.

Figure 6.47 shows the time course of the $y$ coordinate in a typical ciliumcentrosome movie. The two curves exhibit positively-correlated behavior over most of the time course. The amplitude of the cilium fluctuations are greater than those of the centrosome fluctuations, as expected for the extended cilium with its rotational degree of freedom. Interestingly, the two curves cross each other. This, taken with the positively-correlated behavior, suggests that the cilium motion is primarily rotational and that the hinge point lies either at or below the centrosome. If the hinge point were above the centrosome their motion would be anti-correlated, as the cilium and centrosome are physically attached. Purely translational motion would not result in a cross over of the two curves, supporting the idea that the motion observed is rotational. We find it unlikely that the fluctuations are due to thermally induced bending because we never observe bending fluctuations of the cilium so close to the base.

Before analyzing the correlations between cilium and centrosome quantitatively, we first low-pass (LP) filtered the data to reduce high-frequency noise. To filter the data we convolved the time series data with a Gaussian kernel (5 frame (2.5 s) size, 1.5 frame $(0.75 \mathrm{~s})$ width) in Matlab. The results are shown in Figure 6.48. We then determined the PCC discussed above between the LP-filtered cilium and 


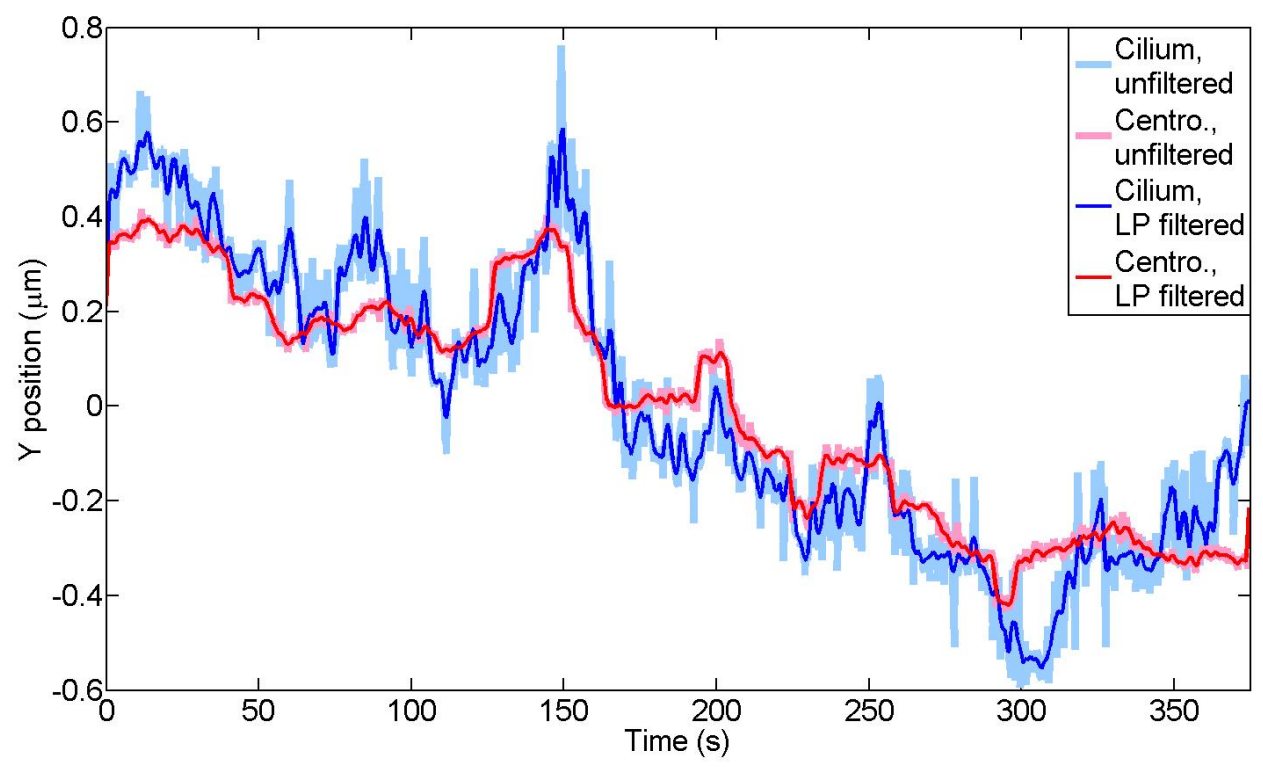

Figure 6.48: Plot of low-pass filtered cilium and centrosome $y$ position vs. time. The dark blue trace tracks the low-pass filtered cilium position and the red trace tracks the low-pass filtered centrosome position. The original, unfiltered signal appears in the background, light blue for the cilium and pink for the centrosome.

centrosome traces for $x-x$ and $y-y$ motions. Figure 6.49 shows cilium $y$ position as a function of centrosome $y$ position for the traces in Fig. 6.48. The linear clustering of the data around a diagonal line of positive slope indicates a high degree of positive correlation, in this case a PCC of $r_{y}=0.89$.

Figures 6.50-6.53 show $x$ and $y$ position time series for four cilium-centrosome pairs and their associated correlation plots. Plots of $x$ position appear on the left while $y$ position plots appear on the right. The analysis is as described above for all the data sets with the exception of the data appearing in the top row of Fig. 6.51 (and the associated correlation plots in the top row of Fig. 6.53). In this movie it was impossible to track $\sim 1 \mu \mathrm{m}$ above the cell membrane, due to background from the cell, so we tracked a point $\sim 2 \mu \mathrm{m}$ above the cell membrane instead. The algorithm also couldn't track the cilium at the end of the time series, and these points were not included in the analysis.

While the majority of the time traces analyzed exhibit positively-correlated behavior, most of the curves are not as strongly correlated as those in Fig. 6.47. Table 6.2 lists the PCC values for the various cilium-centrosome pairs (here the column heading "CC 1" designates the first cilium-centrosome pair presented in the text and so on). The mean values for $x$ and $y$ PCCs are $r_{x}=0.57 \pm 0.34$ and 


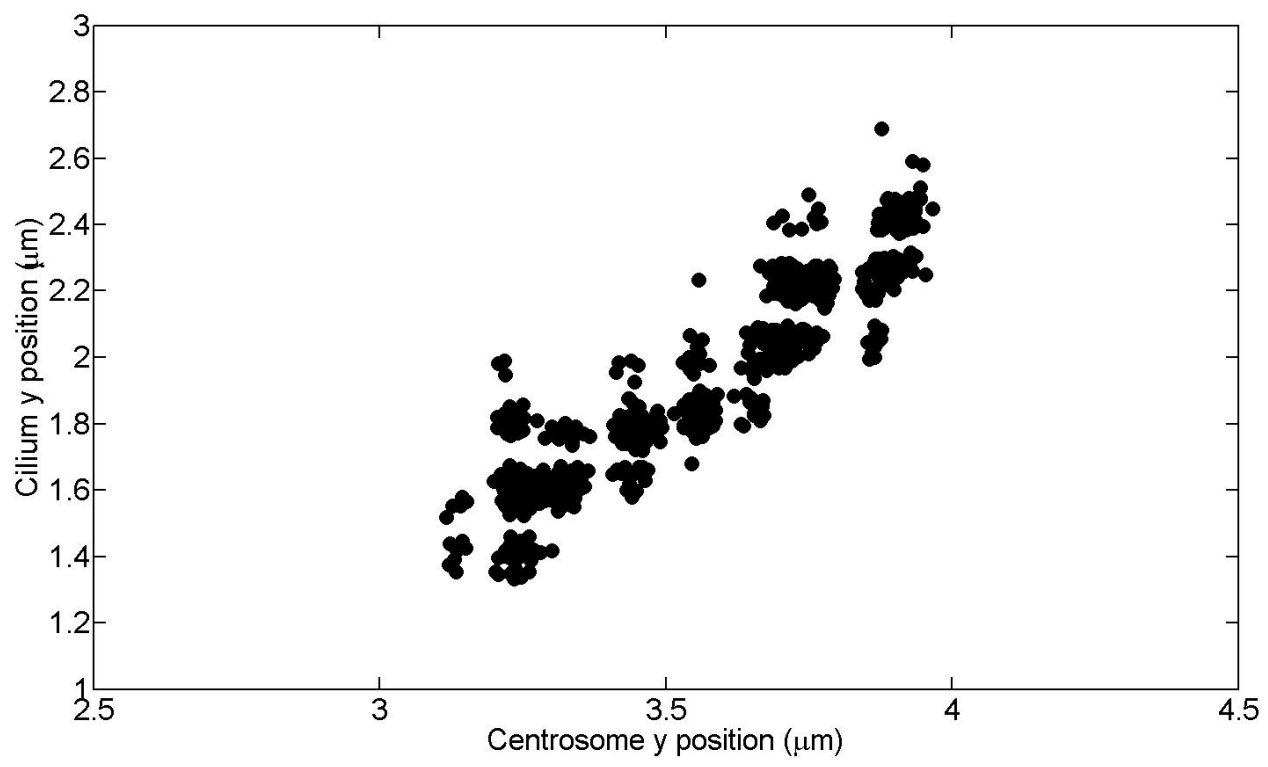

Figure 6.49: Plot of cilium $y$ position as a function of centrosome $y$ position for the traces in Fig. 6.48. The data exhibit a strong positive correlation with a $\mathrm{PCC}=0.89$.
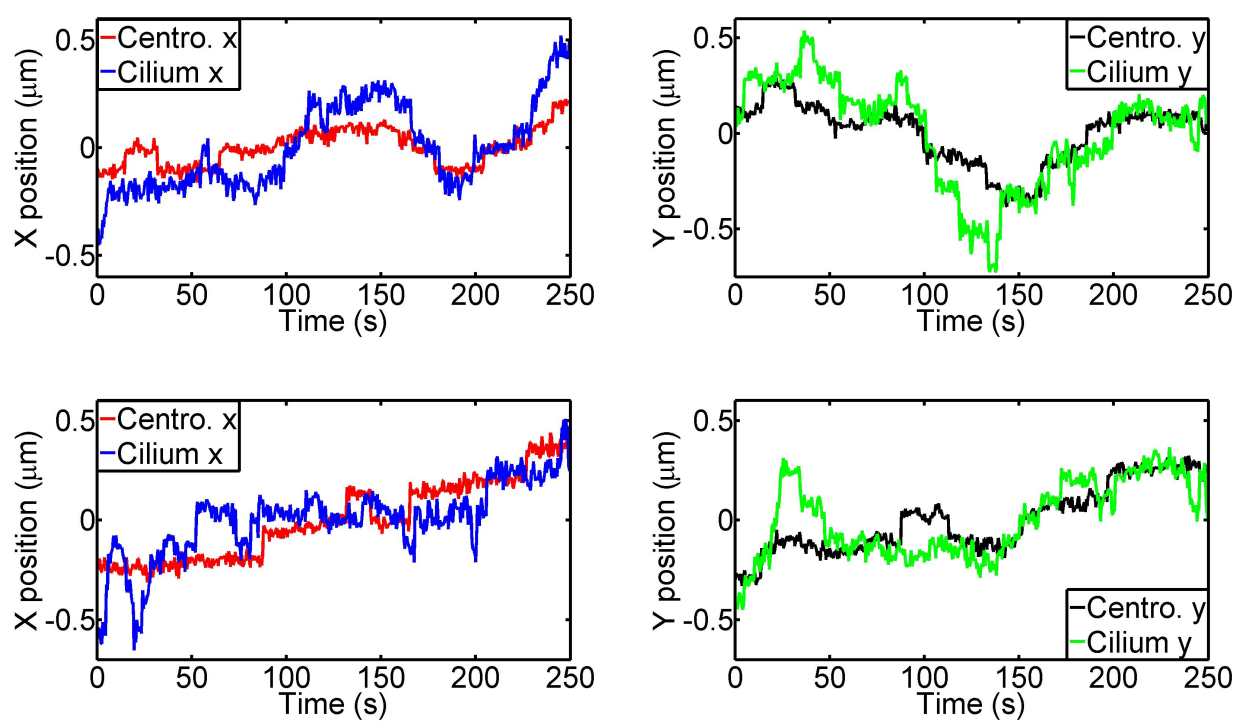

Figure 6.50: Plots of cilium and centrosome position vs. time. The $x$ position (left) and $y$ position (right) of cilium-centrosome pairs is shown for two experiments (top/bottom). The blue and green traces encode $x$ and $y$ cilium position, respectively, while red and black traces represent $x$ and $y$ centrosome position, respectively. 

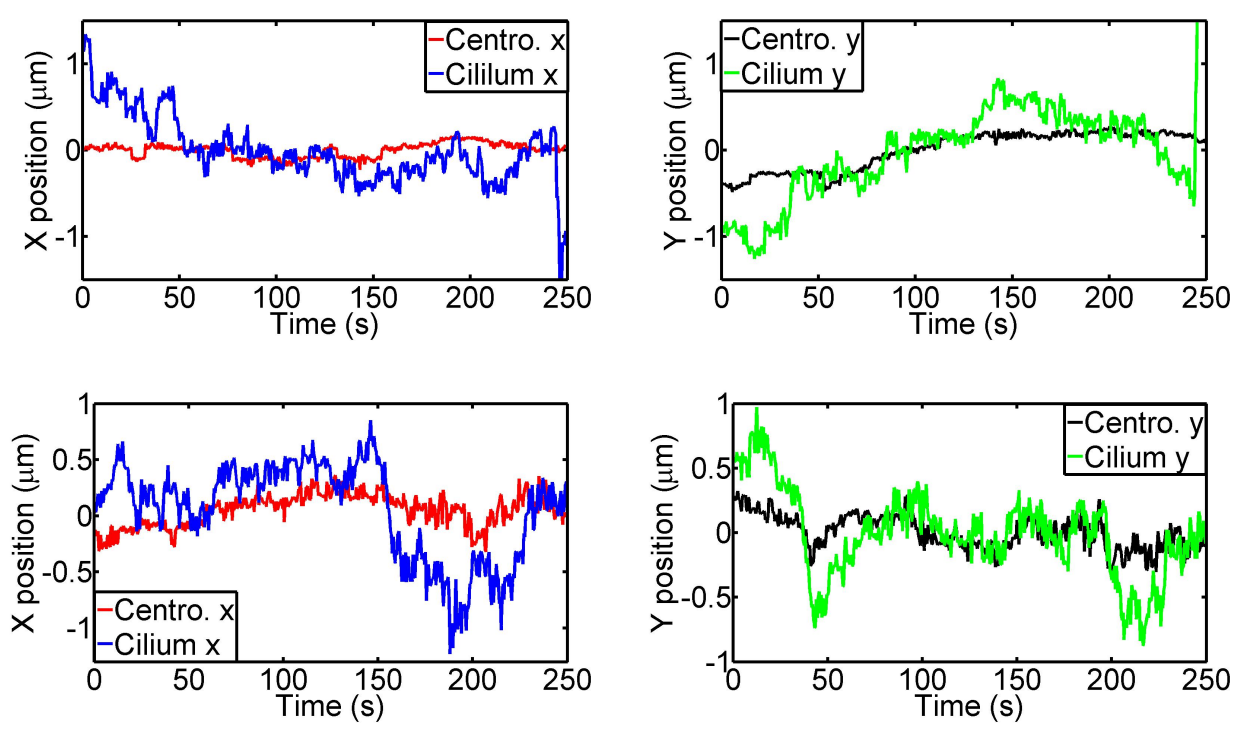

Figure 6.51: Plots of cilium and centrosome position vs. time. The $x$ position (left) and $y$ position (right) of cilium-centrosome pairs is shown for two experiments (top/bottom). The blue and green traces encode $x$ and $y$ cilium position, respectively, while red and black traces represent $x$ and $y$ centrosome position, respectively.
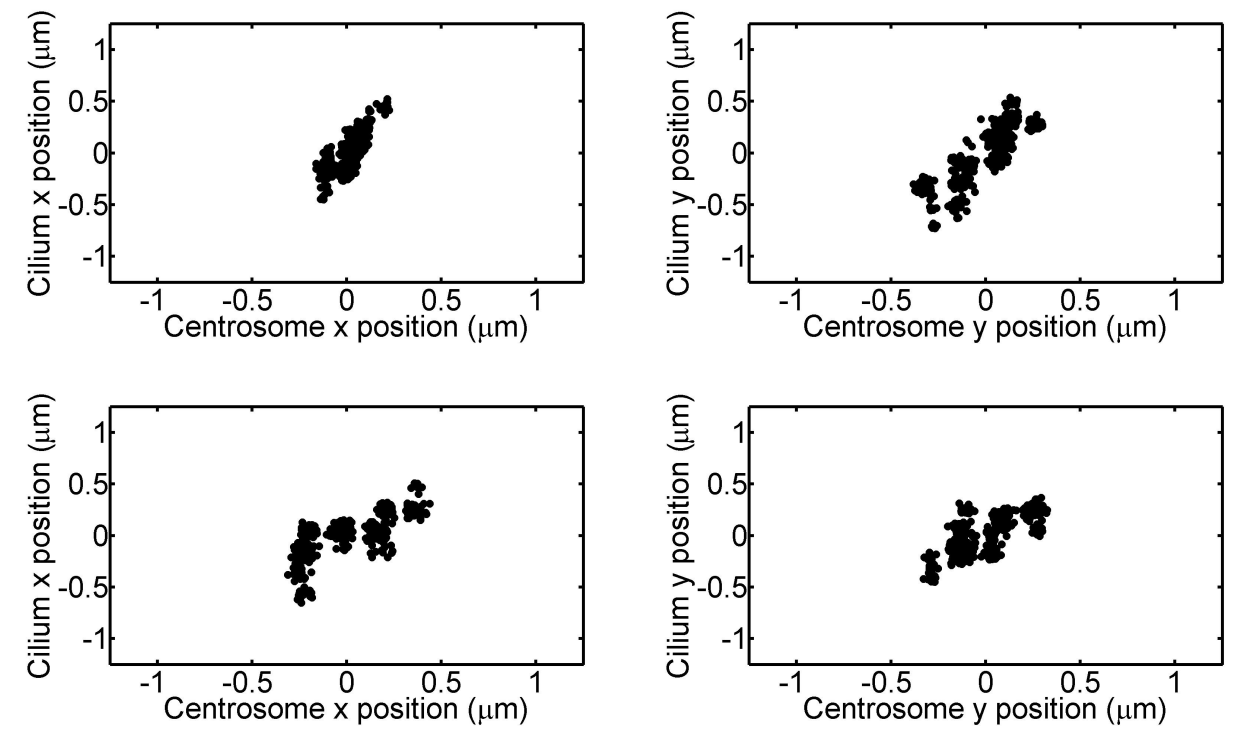

Figure 6.52: Correlation plots of centrosome vs. cilium position for the corresponding panels in Fig. 6.50. 

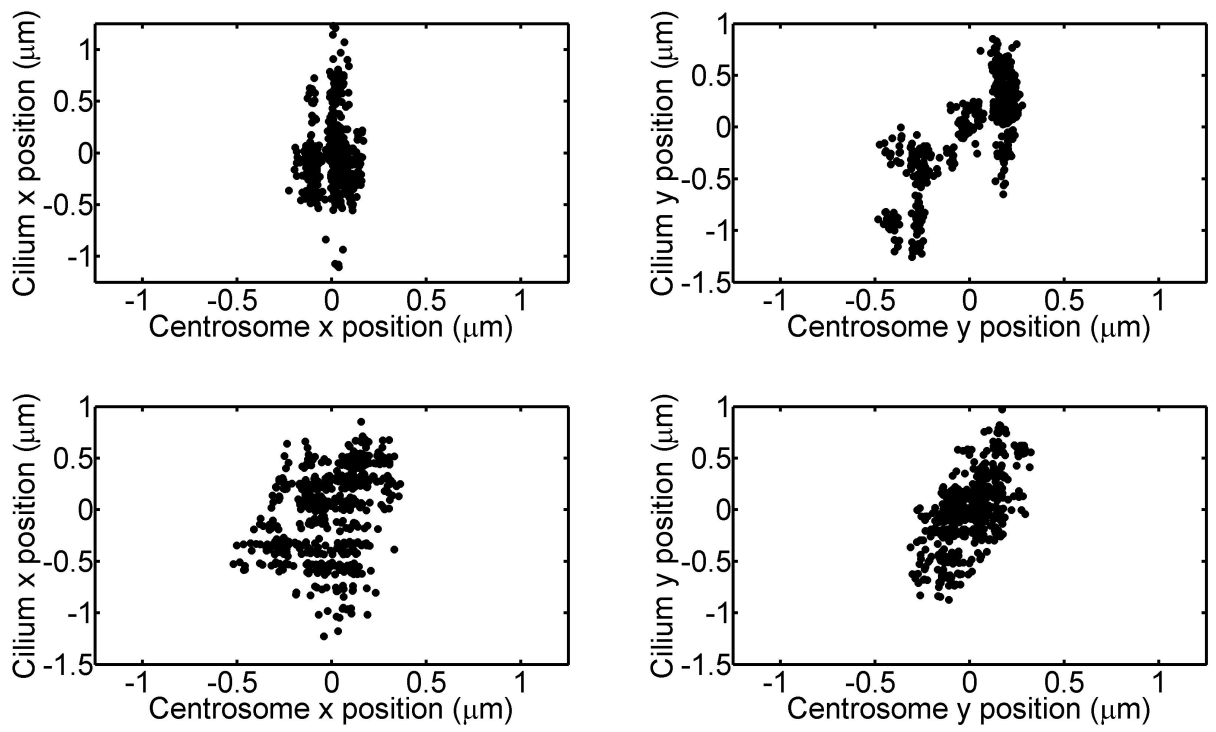

Figure 6.53: Correlation plots of centrosome vs. cilium position for the corresponding panels in Fig. 6.51.

Table 6.2: Correlation coefficient values of cilium-centrosome pairs

\begin{tabular}{|l|c|c|c|c|c|}
\hline & CC 1 & CC 2 & CC 3 & CC 4 & CC 5 \\
\hline Corr. coeff. X pos. & 0.84 & 0.85 & 0.72 & 0.06 & 0.37 \\
\hline Corr. coeff. Y pos. & 0.89 & 0.86 & 0.75 & 0.72 & 0.68 \\
\hline
\end{tabular}

$\left.r_{y}=0.78 \pm 0.09\right)$. The extremely low $x$ correlation value corresponds to the sample which was difficult to track, mentioned earlier, though the $y$ trace from this same experiment is well-correlated. The discrepancy between the $x$ and $y$ values is puzzling, but it could be a result of the small sample size and the relatively large error in the $x$ value. In any case, it would appear that the cilium and centrosome movements are positively-correlated in the majority of the samples analyzed, consistent with the notion that cilium movement is being driven by actin-myosin contractility in the cortex. One can of course ask whether the tail is wagging the dog, i.e. that fluctuations of the cilium are driving the centrosome movements. Though we can't rule this out, it seems unlikely given the previously discussed dependence of the cilium fluctuations on myosin II motor activity.

\section{Errors}

The main potential source of error in these experiments, as in the previous section, is flow. Overall flow in the sample would manifest itself as correlated motion of cilia 
in the FOV, most likely with bending/rotation biased systematically to one side. We looked for correlations between cilia in all samples with multiple cilia in the FOV, and found no correlation. Two of the cilium-centrosome pairs (the first and fourth) came from movies where no other cilia were present in the FOV, so cross correlation analysis between cilia couldn't be performed. In these cases we checked for a preferred direction of bending/rotation in the cilium backbone and found none.

One recently-noticed source of error in the cilium-centrosome analysis is $z$ drift in several of the samples (the second through fourth). We checked the movies for $x y$ motion coupled with the $z$ drift by inspecting the cell sheet by eye for overall motion and found none. While certainly undesirable, it seems unlikely that $z$ drift in itself would lead to spurious correlations between the cilium-centrosome pairs, and could even worsen correlations (e.g. by tracking a higher point on the cilium more subject to thermal fluctuations). Nevertheless we aim to collect more data from cilium-centrosome pairs that avoids this problem. 


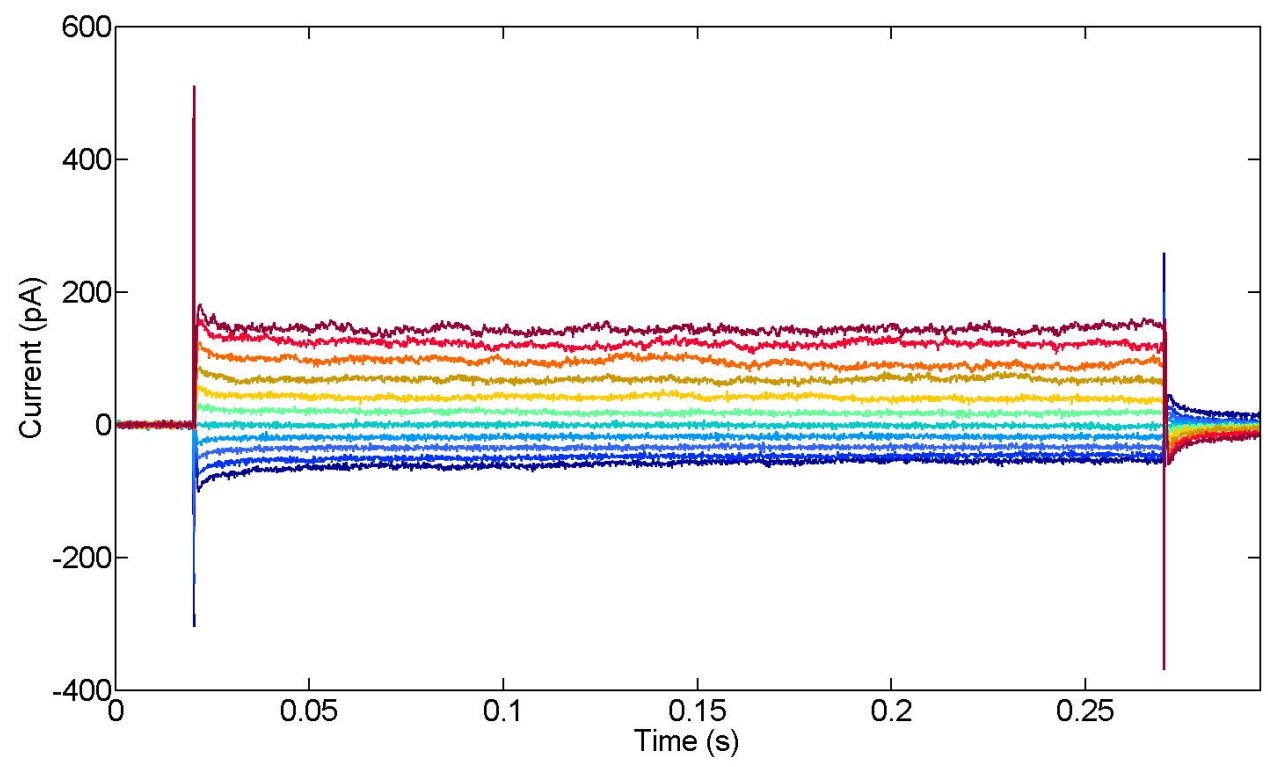

Figure 6.54: Current response of MDCK cell undergoing a voltage ramp protocol. The voltage was clamped between $-120 \mathrm{mV}$ and $+30 \mathrm{mV}$, in increasing $15 \mathrm{mV}$ increments, for a duration of $300 \mathrm{~ms}$.

\section{Electrophysiology}

In the following we describe electrophysiology experiments done in the hope of measuring the mechanosensitive response of MDCK cells. The experiments presented here were done in collaboration with Andreas Neef (Max Plank Institute for Dynamics \& Self-Organization, Bernstein fellow). Unfortunately the experiments below yielded no usable data on this response, for reasons discussed, but we present them here for completeness.

\section{Patch clamp}

As mentioned briefly in Chapter 2, one original goal of the project was to perform a whole-cell patch clamp on individual MDCK cells and look at the electrical response upon mechanical stimulation. To our knowledge, this has never been done, with the only data on the mechanosensitive response coming from imaging of the cells' calcium/nitric oxide response under flow [75]. We found at least one good reason as to why there has been little electrophysiology done on confluent sheets of MDCK cells: when cells are confluent they become very difficult to patch clamp. While the literature is replete with examples of people patch clamping MDCK cells in isolation or separated from a confluent sheet $[91,92,93,94,95]$, we found only two references 


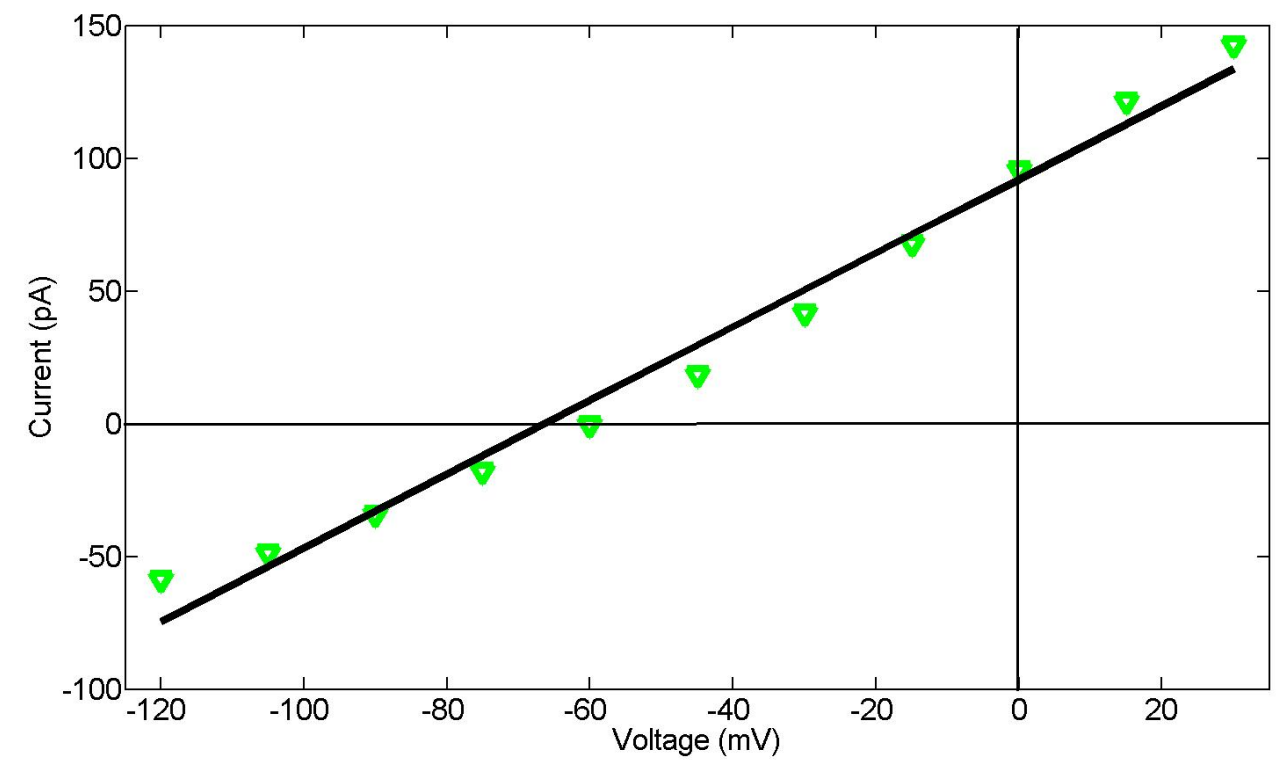

Figure 6.55: Voltage vs. current graph from the protocol performed in Fig. 6.54. The green triangles represent the data and the solid black line is a linear fit with slope $=1.3 \mathrm{nS}$.

in which the experimenters patch clamped confluent MDCK cells [96, 97]. We were, after repeated tries, unable to do the same. Why they become so difficult to patch clamp is unclear to us, though we theorized it may have to do with the glycocalyx, $\mathrm{a} \sim 100 \mathrm{~nm}$ thick coating of sugar chains which glycosolate membrane proteins on the apical membrane of most epithelial cells [98]. We attempted to dissolve the glycocalyx with various treatments (N-glycosidase, trypsin) but we were still unable to patch clamp confluent cells. A day after passaging and in isolation, however, the cells became easy to patch clamp. Figure 6.54 shows the result of a voltage clamp experiment in which the cell was held at a series of incrementally increasing voltages between $-120 \mathrm{mV}$ and $+30 \mathrm{mV}$. The voltage was increased in steps of $15 \mathrm{mV}$ and each voltage step was held for a duration of $300 \mathrm{~ms}$. Figure 6.55 shows a voltage vs. current graph for the same cell. Assuming a liquid junction potential of $-11.9 \mathrm{mV}$ due to our intra/extracellular solutions [99], we can ascertain from Fig. 6.55 that the reversal potential of the cell is $\sim-82 \mathrm{mV}$. The curve appears to follow a GoldmanHodgkin-Katz (GHK) current relationship characteristic of $\mathrm{K}^{+}$channels ${ }^{1}$, possibly due to leak from endogenous "maxi" $\mathrm{K}^{+}$channels [92]. All in all these values are similar to those of human embryonic kidney (HEK) cells, and are what one might

\footnotetext{
${ }^{1}$ The GHK current relationship is not linear and the linear fit in Fig. 6.55 is not meant to represent it.
} 


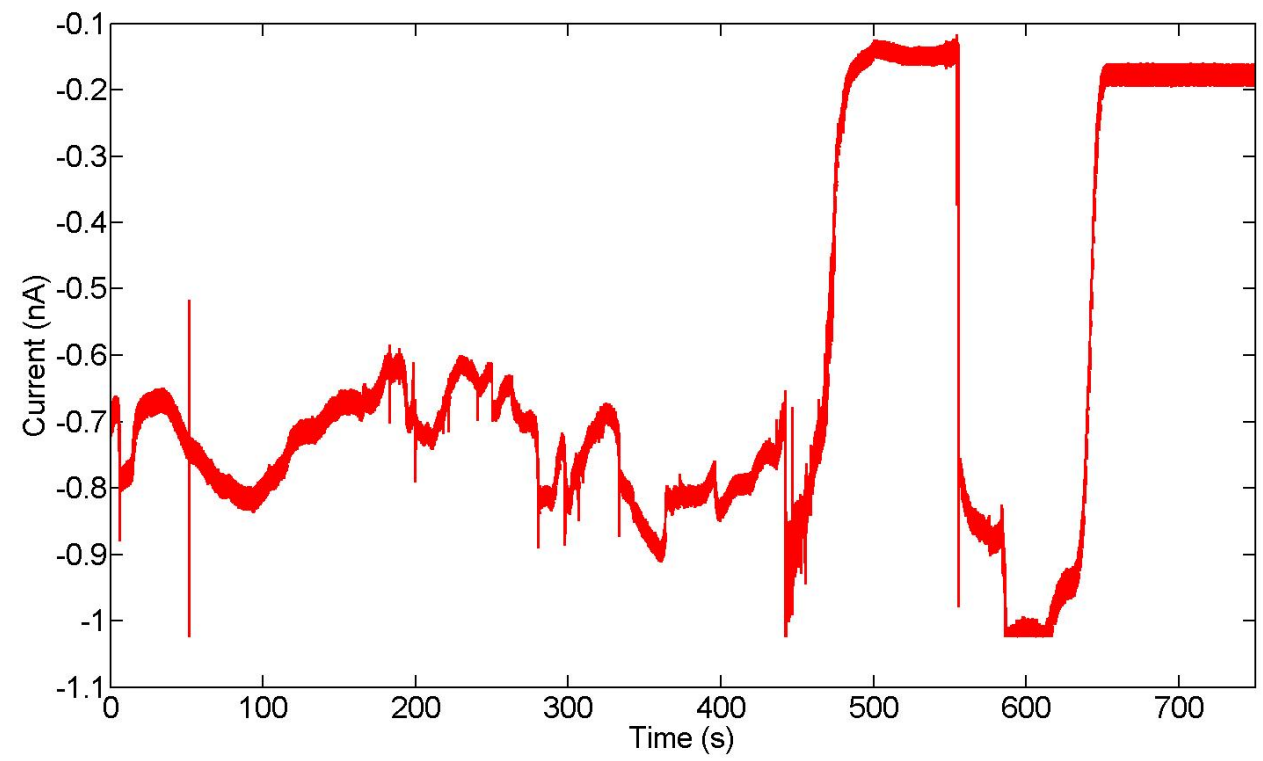

Figure 6.56: Time course of a sharp electrode recording experiment. The current trace exhibits large fluctuations over the course of the experiment, most likely due to an unstable seal. A higher resistance seal only appears at the end of the recording, apparent from the large drop in current amplitude. The micropipette used had a resistance of $\sim 16 \mathrm{M} \Omega$.

typically expect from an epithelial kidney cell line.

\section{Sharp electrode recording}

Due to the difficulty of patch clamping confluent MDCK cells, we decided to try sharp electrode recording, an electrophysiology technique in which one pierces the cell membrane with a very thin, high-access resistance $(\sim 10-50 \mathrm{M} \Omega)$ micropipette to gain electrical access to the cell. The membrane leakage current is mostly occluded by the pipette, but the remaining leakage is higher than in patch clamp experiments, leading to worse current/voltage resolution. Despite initially promising results from cells in a standard, i.e. flat, geometry, in which we were able to achieve a stable recording configuration with small leakage $(\sim 100-110 \mathrm{M} \Omega)$, we were unable to reproduce these results in subsequent experiments in the edgeviewing geometry. Figure 6.56 shows a trace from one such experiment (experiment depicted in Fig. 2.17). The seal between the pipette and cell is extremely unstable, only forming a higher resistance seal at around second 500, then losing the seal 50 seconds later, with the seal re-forming again after another 100 seconds. Additionally, most of the cells that we tried to record from swelled up, probably dying, roughly 
10 minutes after initially beginning to probe them with the sharp electrode. While we don't discount the possibility of successful sharp electrode recordings, more work will be needed to determine if it is an appropriate technique for probing MDCK mechanosensitivity.

\section{Errors}

The main problems we encountered were the infeasibility of patch clamping confluent MDCK cells and the non-reproducibility of our sharp electrode recordings. A stable seal is a prerequisite in the latter for any meaningful measurements, and more work is needed to refine this technique for our purposes. 

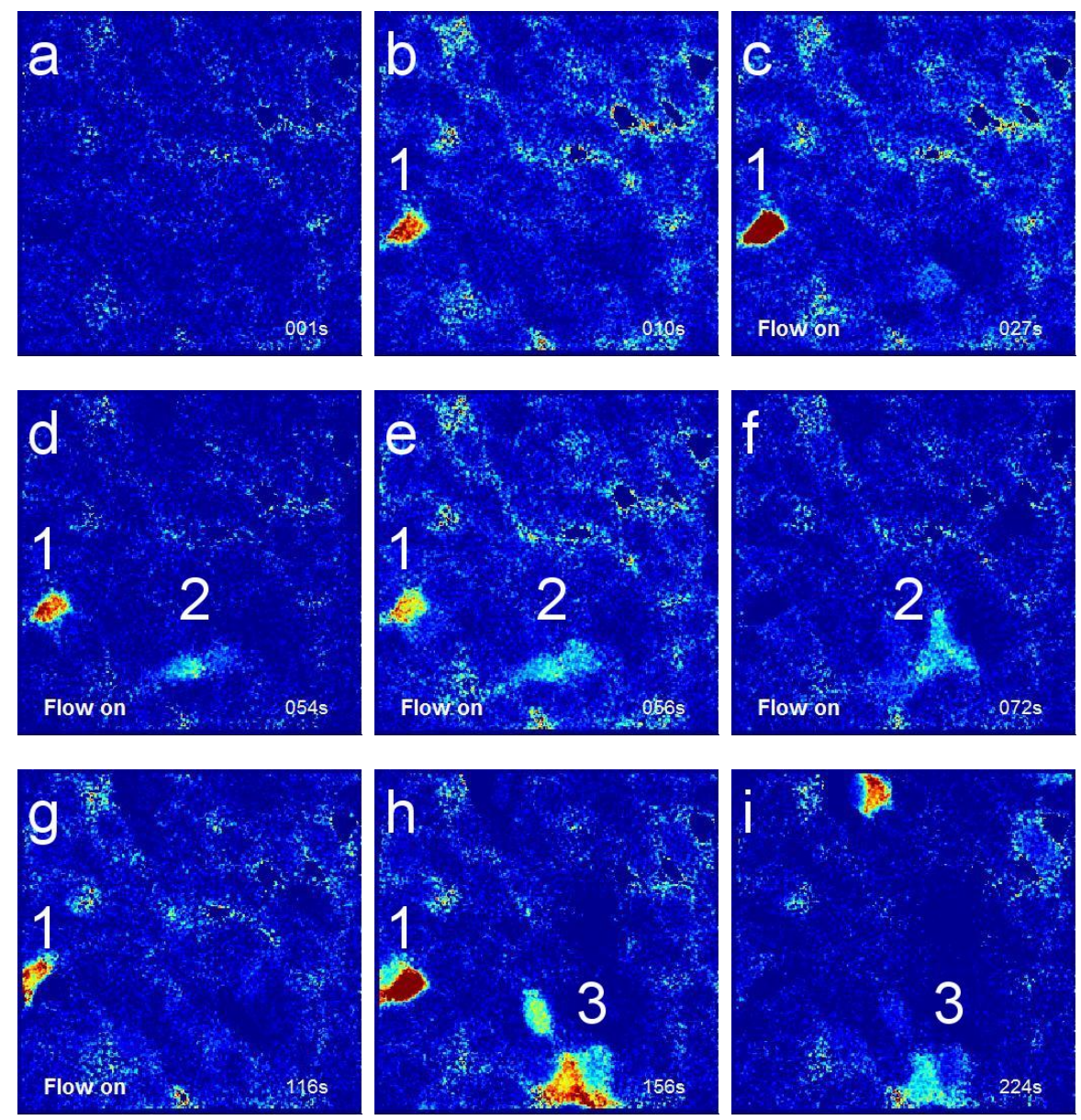

Figure 6.57: Series of stills depicting the ratiometric calcium response of a confluent sheet of MDCK cells loaded with Fura 2-AM dye. The cells are perturbed mechanically through flow from a perfusion system. "Flow on" in the lower left-hand corner designates when the flow is on, and the time stamp in the lower right-hand corner designates total time since the beginning of the movie. The colors scale across the spectrum from blue to red, with deep blue indicating no response and deep red indicating maximum response. Data taken/images created by Andrew Woehler.

\section{Mechanosensitive calcium response}

A flow-dependent, cilium-dependent calcium response is a well-documented phenomenon in kidney epithelial cells $[75,12,13,11]$. Since electrophysiology proved a non-ideal technique with which to measure mechanosensitive signals in MDCK cells, we turned to calcium imaging as an alternative. The following experiments were performed by Andrew Woehler (Max Planck Institute for Biophysical Chem- 

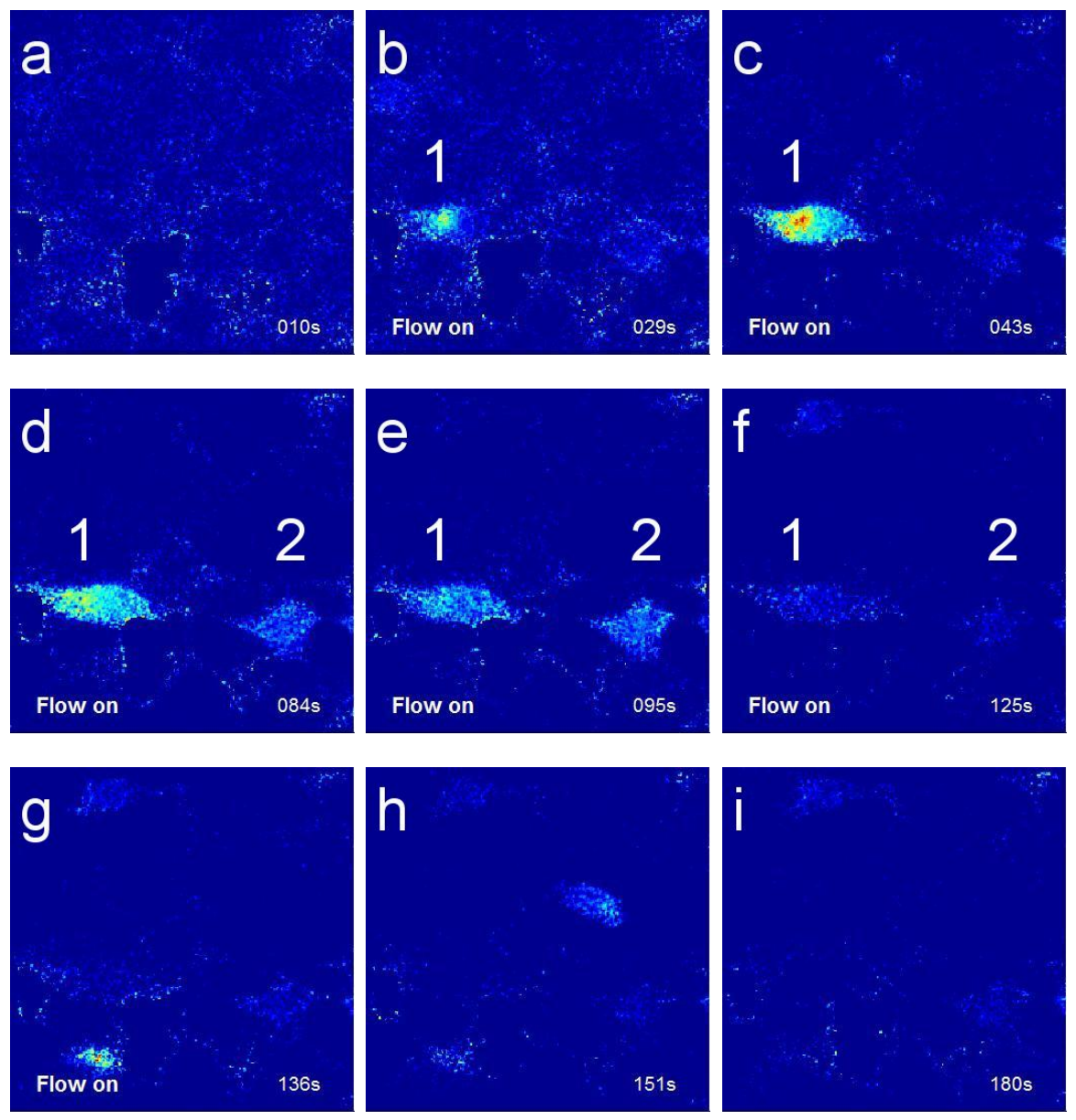

Figure 6.58: Series of stills depicting the ratiometric calcium response of another confluent sheet of MDCK cells loaded with Fura 2-AM dye. In this sample preparation, labeled fluorescent primary cilia were seen to move with the onset of flow. Data taken/images created by Andrew Woehler.

istry, Neher lab). To measure intracellular calcium levels, MDCK cells transfected with a YFP-tagged ciliary protein (Smoothened) were loaded with Fura 2-AM dye. Fura 2 is a commonly used ratiometric dye with a $\mathrm{Ca}^{2+}$-dependent absorption spectrum and an isosbectic point of $\lambda_{e x}=360 \mathrm{~nm}$. Alternating excitation between two wavelengths, in our case $340 \mathrm{~nm}$ and $400 \mathrm{~nm}$, allows quantitative measurements of calcium concentration independent of dye concentration or bleaching. In addition to Fura 2 excitation at $340 \mathrm{~nm}$ and $400 \mathrm{~nm}$, we also excited the sample at $488 \mathrm{~nm}$ in order to visualize the YFP-expressing cilia. Fura 2 and YFP both have emission peaks at $\sim 510 \mathrm{~nm}$. The sample was imaged first for 20 seconds without flow, then for 120 seconds with flow turned on, then for 120 seconds without flow. 

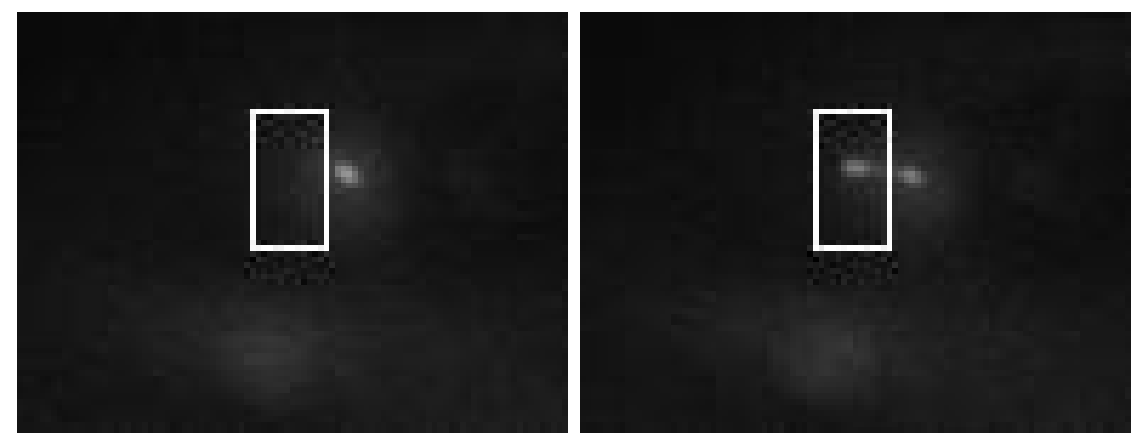

Figure 6.59: Stills depicting cilium movement. The cilium pictured is attached to the cell at position 1 in Fig. 6.58. The cilium's position without flow is shown in the left-hand panel and its position after flow is initiated is shown in the right-hand panel. The white box designates the area in which the average fluorescence was measured

Figure 6.57 shows a series of stills from one such experiment. Here the color represents the change $\Delta R(t)$ in the ratio of the background-corrected fluorescence signals, $R(t)$, in relation to an average of their initial values, $R(0)$, i.e.

$$
\Delta R(t)=R(t)-R(0)=\frac{I(t)_{340}-I(t)_{B g r d 340}}{I(t)_{400}-I(t)_{B g r d} 400}-\sum_{t=1}^{5} \frac{R(t)}{5}
$$

where $I(t)_{340}$ is the intensity of the fluorescence signal in the $340 \mathrm{~nm}$ excitation channel at time $t, I(t)_{B g r d 340}$ is the intensity of the background in the $340 \mathrm{~nm}$ excitation channel at time $t, I(t)_{400}$ is the intensity of the fluorescence signal in the $400 \mathrm{~nm}$ excitation channel at time $t$, and $I(t)_{B g r d 400}$ is the intensity of the background in the $400 \mathrm{~nm}$ excitation channel at time $t$.

Starting in panel b, the cell at position 1 spontaneously fluoresces before the onset of flow and continues fluorescing throughout it. Approximately 50 seconds after flow is initiated the cell at position 2 in panel d starts to fluoresce with the calcium response moving onto adjacent cells over the course of 20 seconds (position2, panels e \& f). Interestingly, there is a large response in several cells (position 3, panels $\mathrm{h} \& \mathrm{i}$ ) which peaks about 40 seconds after flow is stopped. This variety of behaviors, where the response is correlated with turning the flow both on and off, is typical of these experiments.

Figure 6.58 shows a series of stills from an experiment in which calcium response correlated with cilium motion can be observed. Studying cilium movement and calcium response simultaneously would help dissect aspects of mechanosensation such as threshold behavior, low-pass filtering, and velocity dependence. Almost immediately after flow is initiated, in panel b, there is a response from the cell at position 1 which peaks after about 30 seconds. There is a weaker response starting 


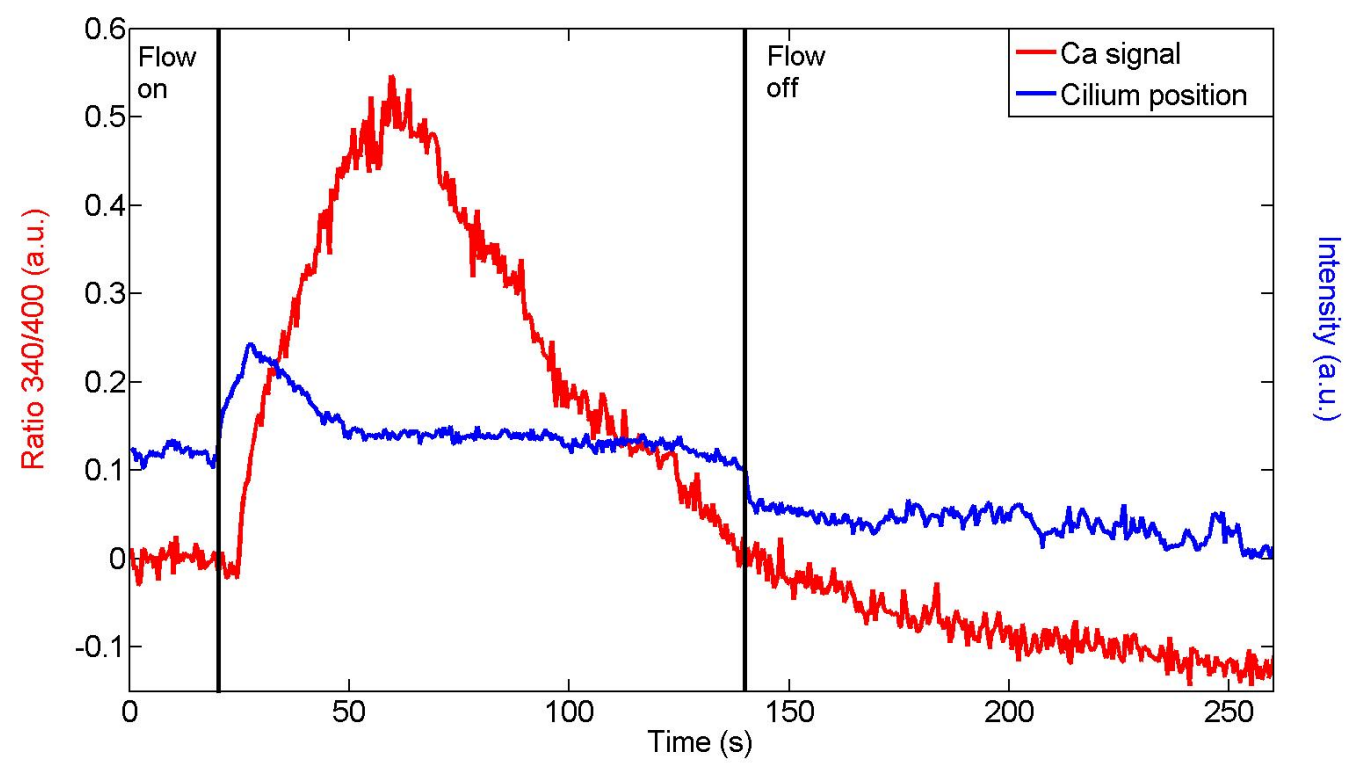

Figure 6.60: Plot of ROI fluorescence intensity/calcium level vs. time. The average ROI intensity (blue) is a proxy for cilium position and the ratiometric Fura 2 signal (red) is calculated per Eqn 6.7. These traces correspond to the cell at position 1 in Fig. 6.58.

in panel $\mathrm{d}$ from a cell at position 2, which peaks approximately 70 seconds after flow is initiated. On both of the cells fluorescent cilia can be observed to move with initiation and cessation of flow. Due to the fact that the focal plane of the microscope doesn't change in these experiments (it is focused slightly above the cell surface, to make both the cytosolic increase in calcium and the cilium visible), it is impossible to accurately follow the dynamics of the cilium. We can, however, get a gross measure of cilium motion by way of the method illustrated in Figure 6.59. Upon initiation of flow, both of the cilia in question move from their initial, "straight" configuration (left-hand panel) to an extended configuration which appears to be more flattened out against the cell membrane (right-hand panel). The cilium moves from its initial configuration to its flattened configuration quickly, in the space of a few frames. In order to estimate the cilium movement's time course, we tracked the average fluorescence in a boxed region-of-interest (ROI) next to the cilium's initial position (delineated by a white square in Fig. 6.59). Initially there is only background fluorescence in this square, but upon initiation of flow the extended cilium tip crosses into this ROI, increasing the average fluorescence. Upon cessation of flow, the cilium moves back to its original configuration, and there is a concomitant drop in the boxed area's average fluorescence. 


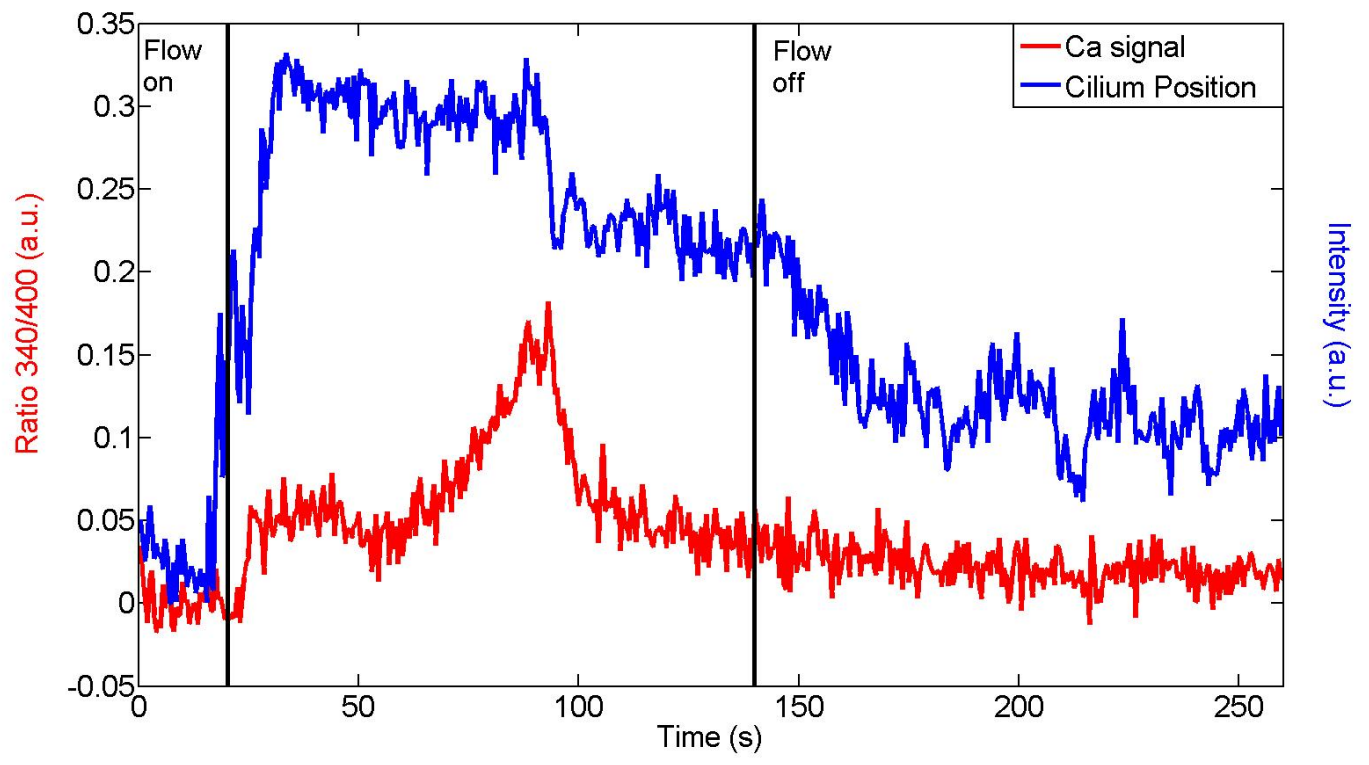

Figure 6.61: Plot of ROI fluorescence intensity/calcium level vs. time. The average ROI intensity (blue) is a proxy for cilium position and the ratiometric Fura 2 signal (red) is calculated per Eqn 6.7. These traces correspond to the cell at position 2 in Fig. 6.58.

With this gross estimate of cilium position in hand, we can compare the cilia movements to the calcium responses shown in Fig. 6.58. Figures $6.60 \& 6.61$ show the average fluorescence in the ROI neighboring the cilium initial position and the calcium response of the cells at positions 1 and 2 in Fig. 6.58, respectively. In Fig. 6.60 we see an almost simultaneous cilium movement and rise in calcium levels, beginning immediately with the onset of flow. In Fig. 6.61 the cilium movement again begins with the onset of flow and quickly reaches its extended position, but the calcium response is greatly delayed. While there is a small initial increase correlated with cilium movement, the increase plateaus for roughly 30 seconds before the more typical calcium response peak begins. Previous studies have found that the calcium response of MDCK cells typically peaks $\sim 25$ seconds after the onset of flow [14]. While the results presented here are encouraging, better visualization of the primary cilium shape and position, either via confocal stacks or an edge-viewing geometry, is necessary to make the connection between cilium dynamics and calcium response, a challenge we hope to address in future work. 


\section{Errors}

The main constraints of these experiments consist of the aforementioned confinement to one viewing plane, due to the microscope type and geometry, and only coarse control over flow. Currently flow is generated by a gravity-driven perfusion system and it is difficult to quantify the fluid flux it generates in the sample. In the future we would like to build either flow chambers capable of delivering controlled laminar flow or to manipulate the cilium directly with an optical trap. In either case we would mount the sample in either an edge-viewing geometry or use a confocal microscope in order to track the cilium shape and movement while at the same time monitoring the calcium response. 


\section{Chapter 7}

\section{Discussion}

In this final chapter we discuss the implications of the main results presented in the previous chapter. Here we only address results from the bending stiffness experiments and the discovery of actively driven fluctuations in the primary cilium. While there has been little work done, to our knowledge, directly correlating cilium movement and calcium response, making the final results in the last chapter potentially interesting, these findings are still very preliminary. We first present the bending stiffness results in the context of similar measurements in the literature and discuss their implications. Second, we give a brief overview of the cilium attachment to the cell and discuss the active fluctuations in this context. Finally we give an outlook on future work to follow up on the findings presented here.

\section{Cilium mechanics}

To put the bending stiffness results quoted in the last chapter in perspective, let's first consider the structural makeup of the cilium. Figure 7.1 shows diagrams of both motile and non-motile (primary) cilium cross sections ${ }^{1}$. Even the most cursory inspection reveals the primary cilium to be bare in comparison with the motile cilium. Motile cilia, in addition to having a central microtubule pair lacking in primary cilia, have a host of accessory proteins: inner and outer dynein arms, nexin linkers, and radial spokes [100]. The dynein arms generate relative sliding between microtubule doublets which gives rise to the characteristic beating motion of motile cilia [101]. The nexin linkers prevent the microtubule doublets from sliding more than a given distance relative to each other, while the radial spokes and central microtubule pair are thought to be involved in coordinating the motion between the various doublets $[101,102,103]$. Unlike motile cilia, the primary cilia lack this

\footnotetext{
${ }^{1}$ Though motile primary cilia do exist, one example being embryonic nodal cilia, here we will use primary cilia to mean the more common, non-motile variant, the only kind examined in this work.
} 
non-motile cilium
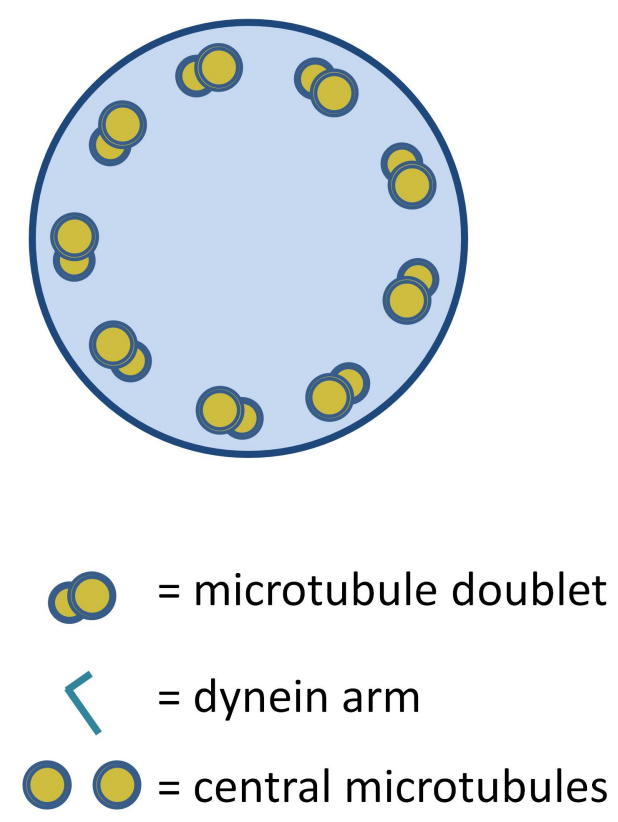

motile cilium

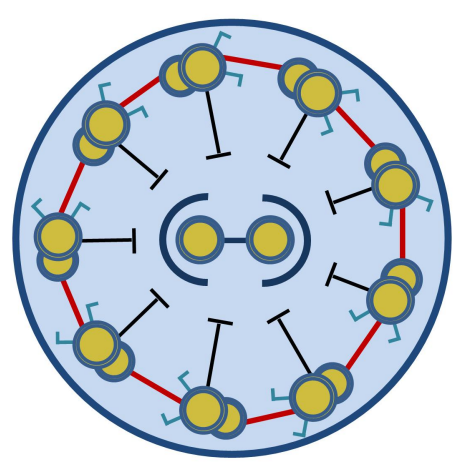

T = radial spoke

$=$ nexin

Figure 7.1: Diagrams of non-motile (left) and motile (right) cilium cross sections. Primary cilia are generally non-motile and have a so-called " $9+0$ " axonemal structure, lacking the central microtubules present in motile cilia. Motile cilia have a "9+2" axonemal structure, which includes a central microtubule pair, as well as radial spokes, inner and outer dynein arms, and nexin linkers.

plethora of protein cross links. The key feature that both share are the nine microtubule doublets arranged in a columnar geometry, which provide the cilium with structural support. Given the bending stiffnesses of the main structural elements of the cilium, i.e. microtubule doublets, and their geometrical arrangement, we can estimate the bending stiffness that a cilium should have. Though little is known about the bending stiffness of microtubule doublets, the bending stiffness of microtubules is an area of active research and debate, with reported stiffnesses ranging over an order of magnitude, from $0.1-3.4 \times 10^{-23} \mathrm{~N} \cdot \mathrm{m}^{2}$. Reference [81] even reports a length dependent microtubule bending stiffness that ranges from $0.05-2.1 \times 10^{-23} \mathrm{~N} \cdot \mathrm{m}^{2}$, increasing with increasing microtubule length. Table 7.1 summarizes some of the values found in the literature. While microtubules' wide range of flexural rigidity values make a precise prediction of the cilium's bending stiffness impossible, we can do the next best thing and estimate a range in which it should fall. Given a bundle of $N$ filaments of bending stiffness $E I_{f}$ arranged in a columnar geometry, the bending stiffness of the bundle, $E I_{B}$, scales as [109] 
Table 7.1: Literature values for microtubule bending stiffness

\begin{tabular}{|c|c|}
\hline Reference & $E I\left(\times 10^{-23} \mathrm{~N} \cdot \mathrm{m}^{2}\right)$ \\
\hline van den Heuvel et al. $[104]$ & $0.10 \pm 0.01$ \\
\hline Felgner et al. $[78]$ & $0.19 \pm 0.01$ \\
\hline Venier et al. $[105]$ & $0.49 \pm 0.04$ \\
\hline van Mameren et al. $[106]$ & $0.61 \pm 1.3$ \\
\hline Gittes et al. $[80]$ & $2.2 \pm 0.1$ \\
\hline Mickey et al. $[107]$ & $2.6 \pm 0.2$ \\
\hline Dogterom et al. $[108]$ & $3.4 \pm 0.7$ \\
\hline
\end{tabular}

$$
E I_{B} \propto N^{\alpha} E I_{f}
$$

where $1 \leq \alpha \leq 3$ is an exponent dependent on inter-filament cross linking. In the limit of weak to no cross linking $(\alpha=1)$ the bending stiffness of the bundle simply scales with the number of filaments, whereas in the strongly coupled regime $(\alpha=3)$ the bending stiffness scales with the cube of the number of filaments. Thus filament cross linking clearly has a huge impact on the overall stiffness of the bundle.

Figure 7.2 plots the values that we and others have found for primary cilia bending stiffness, as well as a sperm flagellum bending stiffness value for comparison. The "theoretical min/max" values (dark blue lower/upper lines) are calculated using the microtubule data in the preceding table in the weakly/strongly coupled limits. Specifically, "theoretical min" is calculated by assuming that each microtubule doublet has the same bending stiffness as a single microtubule, that this bending stiffness is equal to the lowest value quoted in Table 7.1, and that the individual filaments are weakly coupled, i.e. $E I_{B}=N E I_{f}$. The "theoretical max" value is calculated by assuming that each microtubule doublet has twice the bending stiffness of a single microtubule, that a single microtubule has a bending stiffness equal to the highest value quoted in Table 7.1 , and that it has a maximal stiffness, $E I_{B}=\left[1+2 / \sin ^{2}(\pi / N] E I_{f} \approx 22 N E I_{f}\right.$, per Ref. [109].

Several things are apparent from Figure 7.2. First, the measurements for primary cilium flexural rigidity cluster to within a factor of $\sim 5$, with the exception of the experiments in Downs et al.. Indeed, in the experiments by Young et al., while they quote a mean value of $8.4 \times 10^{-23} \mathrm{~N} \cdot \mathrm{m}^{2}$ for the flexural rigidity, they mention that $70 \%$ of their data falls in the bounds of $1-5 \times 10^{-23} \mathrm{~N} \cdot \mathrm{m}^{2}$, encompassing the range of values found in this work and by Schwartz et al.. The experiments described in Downs et al., and Young et al. were conducted by the same group using the same technique and the reason for the large divergence in values is unclear. Our values, the values found in Schwartz et al., and those in Young et al. sit close to the "theoretical min" line, suggesting that a) the microtubule doublets are not 


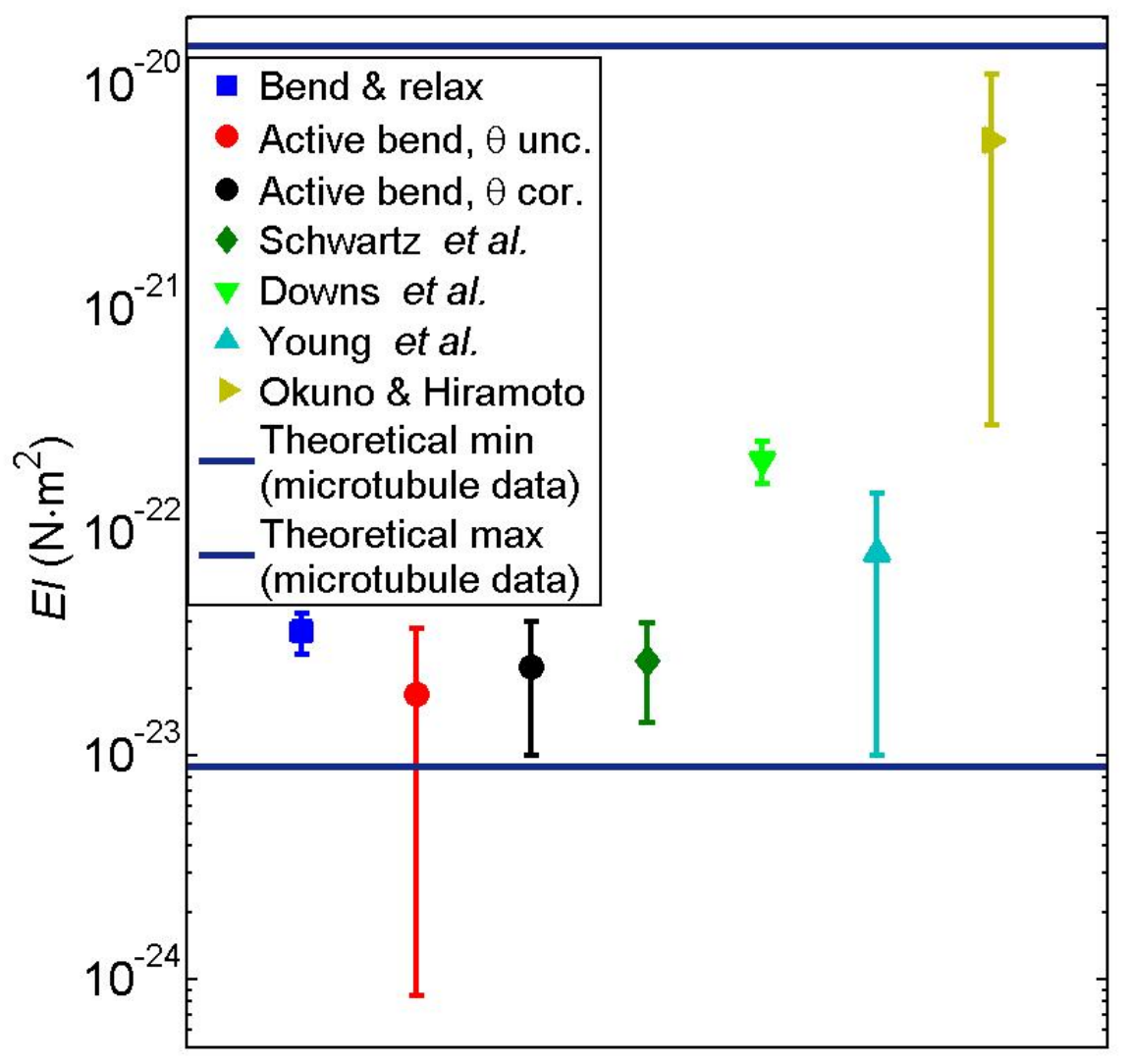

Figure 7.2: Bending stiffness values for primary cilia in renal epithelial cells. Depicted are the values presented in this work (bend \& relax and active bending experiments) as well as those from Schwartz et al. [17], Downs et al. [18], and Young et al. [19]. The bending stiffness value from Okuno and Hiramoto [110] is for echinoderm sperm flagella and is shown for comparison. The "active bend, $\theta$ unc." refers to the average of the middle row of values in Table 6.1, which are uncorrected for rotation angle, while "active bend, $\theta$ cor." refers to the average of bottom row of values in Table 6.1, which are corrected for rotation angle. The "theoretical min/max" values are calculated as described in the text. 
much stiffer than the softer values recorded for single microtubules and b) that the doublets in the cilium are weakly coupled. This stands in stark contrast to the bending stiffness values for the motile, highly cross-linked sperm flagella from Okuno and Hiramoto, which are more than a hundred times stiffer. Our measurements imply that $E I_{\text {doublet }} \approx 0.4 \mathrm{x} 10^{-23} \mathrm{~N} \cdot \mathrm{m}^{2}$, which is $\sim 3$ times softer than the only measured value that we're aware of in the literature (Ref. [111] quotes a value of $1.4 \times 10^{-23} \mathrm{~N} \cdot \mathrm{m}^{2}$, measured from the deformation of demembranated doublets from sperm flagella in flow). If we use our estimated value of microtubule bending stiffness in the strongly coupled limit to estimate sperm flagella stiffness we come up with a value that falls in the middle of the flagellum stiffness values in the presence of $\mathrm{ATP}^{2}$ reported in Refs. [110, 111, 112]. This estimate only takes into account the bending stiffness due to the outer microtubules doublets and ignores the contribution from the radial spokes and central microtubule pair. Given the large variance in reported microtubule values and the structural differences between primary and motile cilia this agreement is somewhat surprising and reassuring.

The weak coupling between doublets is consistent with EM studies of cilium structure, in which the doublets have been observed to lose their relative orientation and migrate inward towards the center of the cilium [113]. Reference [113] found instances in which microtubule doublets didn't continue for the whole length of the cilium, in some cases resulting in tapering cilia tips with only four doublets instead of the usual nine. The authors of Reference [76] noted a mismatch between their model of a primary cilium bending profile in flow, which assumes a constant $E I$, and observed cilia bending profiles. They noted that the cilium appeared stiffer at the base and that the change in $E I$ is more pronounced in longer cilia, which is consistent with the loss of ciliary order just described. Such structural caveats caution against the assumption that cilium flexural rigidity is necessarily uniform along its length. Indeed, preliminary results reported in Reference [85] indicate signs of cilium stiffening upon repeated exposure to high flow rates, hinting that cilium mechanical properties may even change over time.

Physiologically speaking, the relative softness of primary cilia makes sense given their mechanosensory role. If cilium bending is the key variable in eliciting mechanosensitive response, then an easily bent sensor is more sensitive to flow than a stiff one. The corollary to this is that an easily bent cilium would transmit force to mechanosensitive channels located at the base more poorly and more slowly than a stiff one. It has been shown that polycystin-1 (PC1), an accessory protein to the mechanosensitive channel polycystin-2 (PC2), thought to be the main mediator of mechanosensation in the primary cilia, localizes to the basal body [114]. Other immunofluorescence results, however, have shown PC1 and PC2 localized on the primary cilia [14]. This has led some to claim that mechanosensation happens at

\footnotetext{
${ }^{2}$ in the absence of ATP flagellum stiffness values increase 10 -fold.
} 
the base $[76,114]$ through forces transmitted by the cilium, while others claim that cilium bending gates channels present in the ciliary membrane [14,77]. The authors of Ref. [76] performed finite element simulations which predicted maximal membrane stress for a cilium at its base and a buildup time of 20 seconds before the maximal stress was reached. This is in line with the observed lag between the start of flow and the maximal calcium response, which the authors of Ref. [76] took as support for the hypothesis that mechanosensation occurs via channels located at the base. Clearly cilium flexural rigidity values alone won't solve this debate, and more work is needed to disentangle the interplay between the cilium's mechanics and its mechanosensitive response.

\section{Active fluctuations}

The most novel result presented in this work is the demonstration of active fluctuations in the primary cilium. To our knowledge, this is the first time this phenomenon has been reported. Given that the fluctuations are connected to myosin activity and that they appear primarily as angular rotations, it's worth reviewing what is known about the attachment of the primary cilium at the base, to see what's going on "below the tip of the iceberg".

Figure 7.3 shows a diagram of the cilium base and its anchorage to the cell. During ciliogenesis, the basal body (mother centriole) moves to the cell surfaces and integrates itself into the cell cortex [84]. Microtubule doublets start elongating from the basal body outward towards the apical side of the cell, apparently using the " $9+0$ " arrangement of microtubule triplets in the basal body as a template for cilium geometry. Two to five basal feet extend outwards from the basal body and act as anchorage points for outward radiating cytoplasmic microtubules [115]. The basal feet are thought to play a role in stabilizing and anchoring the cilia to

the cell [85]. Extending from the proximal side of the basal body is a system of filamentous structures called striated rootlets, whose exact function is unknown but which link the centrosome to the nucleus [115]. It's hypothesized that the striated rootlets exert contractile forces via cytokeratins, a type of intermediate filament to which they associate, and that their tight link with the nucleus plays a role in nuclear positioning and/or cell cycle timing [85, 115]. Extending from the basal body (or a striated rootlet) is a filamentous structure which connects it to the daughter centriole, termed the striated connector [116]. Together the basal body and daughter centriole make up the centrosome, which is the main microtubule organizing center of the cell.

Given the integration of the centrosome within the cytoskeleton, with connections to all the various cytoskeletal filaments, its perhaps not surprising that we see active fluctuations in the primary cilium. Actin-myosin contractility is a well- 


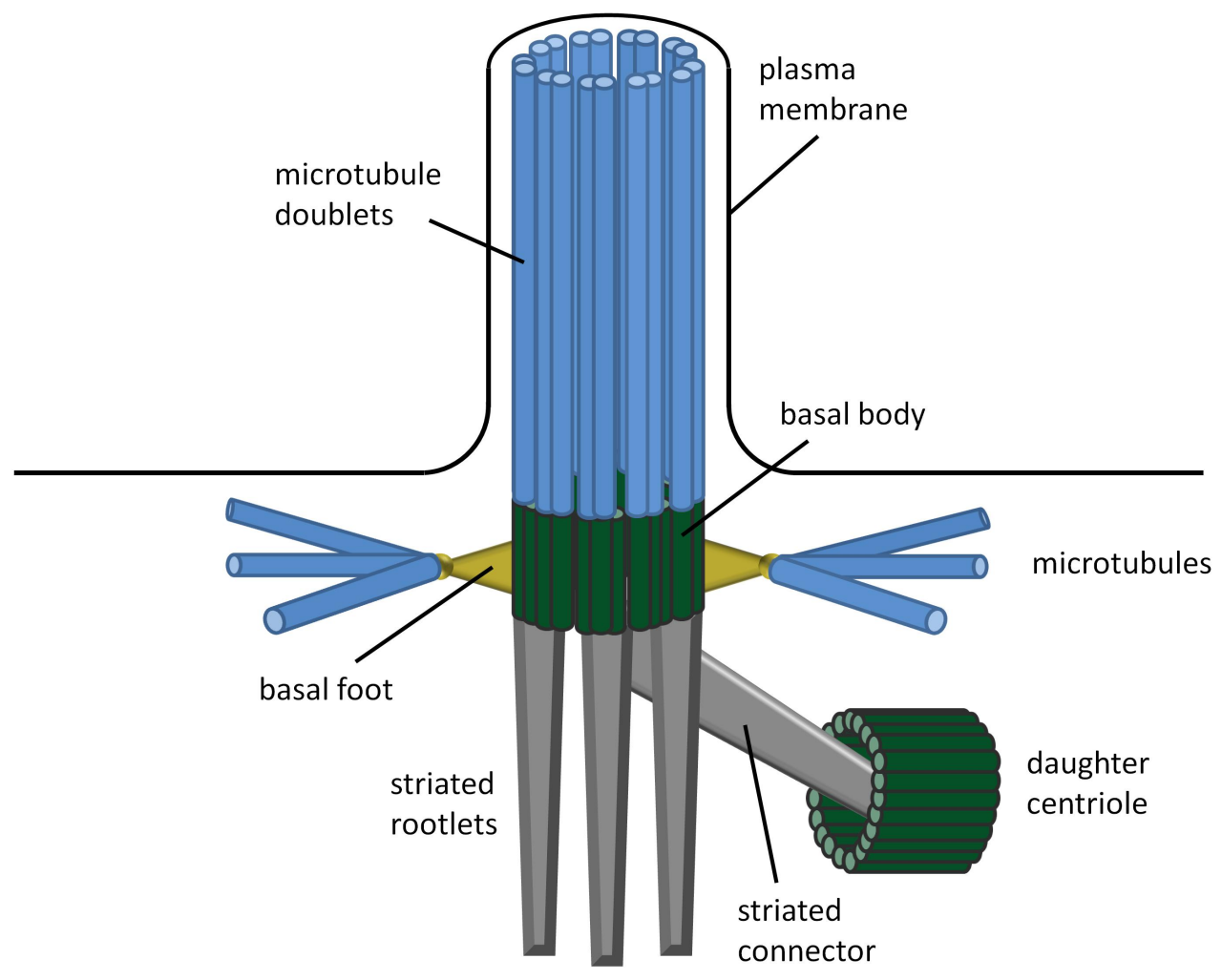

Figure 7.3: Diagram of the primary cilium base and its attachment to the cell.

known force generator in the cell and the fluctuations of microspheres embedded in in vitro actin-myosin networks have been shown to reflect the network's active dynamics [26]. Since the cilium base is embedded in such an active network with a limited hinge-like degree of freedom, its motion could be acting as a reporter of the underlying non-equilibrium cytoskeletal dynamics.

From a biological standpoint its tempting to ask if there is any possible physiological function mediated by the cilium fluctuations. The link between cilium mechanosensing/cilium length regulation and cytoskeletal dynamics is well documented $[117,118,119]$. A plethora of different stimuli have been shown to modulate cilium length, a short list of which is given in Table 7.2. Most interestingly in light of our results, silencing the protein ACTR3, necessary for nucleating actin polymerization, or inhibiting actin polymerization with cytochalasin D resulted in increased cilium length and promoted ciliogenesis [118]. The authors of Reference [120] found that both inhibition of actin polymerization and promotion of actin stabilization by jasplakinolide treatment led to increased cilium lengths, and that both effects were correlated with increases in the level of soluble intracellular tubulin. Inhibition of actin-myosin contractility however has been shown to have no effect on cilium 
Table 7.2: List of factors known to influence cilium length (from Ref. [119])

\begin{tabular}{|c|c|}
\hline Length increase & Length decrease \\
\hline $\begin{array}{c}\text { actin depolymerization/ } \\
\text { actin stabilization }\end{array}$ & $\begin{array}{c}\text { ACTR3 (actin } \\
\text { branching protein })\end{array}$ \\
\hline $\begin{array}{c}\text { increase in cyclic AMP/ } \\
\mathrm{Gd}^{3+}\left(\mathrm{Ca}^{2+} \text { blocker }\right)\end{array}$ & $\mathrm{Ca}^{2+}$ influx \\
\hline increased soluble tubulin & microtubule stabilization \\
\hline $50 \mathrm{mM} \mathrm{LiCl,vKCl,} \mathrm{NaCl}$ & \\
\hline renal injury & \\
\hline
\end{tabular}

length [121, 122], implying that cilium fluctuations likely have no role in length regulation.

Intriguingly, global cytoskeletal mechanics have been shown to effect the mechanosensitive calcium response of kidney epithelial cells [117]. The authors of Reference [117] found that inhibition of myosin motor activity indirectly via Y27632, a rho-associated kinase inhibitor, abolished flow-induced calcium response. This was not unique to myosin motor activity, however; disruption of the actin network via cytochalasin B and latrunculin B, disruption of the microtubule network with colchicine or nocodazole, stabilization of microtubules via taxol, and disruption of integrinbinding via GRGDSP were all found to abolish the flow response. Apparently ciliary mechanosensation is a finicky and sensitive phenomenon with respect to overall cellular mechanics.

Giving ourselves over to speculation for a moment, it seems conceivable that the stiff-rod, rocking back and forth motion of the cilium fluctuations could create stress in the plasma membrane/cytoskeleton at the cilium base. Given the possibility that ciliary mechanosensation is mediated by base-located ion channels, membrane prestress induced by fluctuations could act to sensitize channel gating or perhaps gate channels directly. This would be akin to the phenomenon of stochastic resonance, in which the optimal signal-to-noise ratio of a non-linear system's response corresponds to a non-zero noise level, an effect which has been demonstrated in various biological systems $[123,124]$. Demonstrating this in the cilium would be challenging to say the least, given the tangle of factors that effect calcium signaling. One interesting possibility would be to turn the problem on its head and look for feedback response between mechanosensitive activity and cilium fluctuations, examining for example the effect of channel blockers on cilium fluctuations. Such an approach would be a logical and achievable extension of the research presented here. 
One final variable of interest is cilium orientation, which is affected dramatically by sub-ciliary mechanics and actin-myosin dynamics. In post-menopausal women it has been shown that the striated rootlets of human oviduct epithelial cells shorten and that the cilium rotates its orientation by $90^{\circ}$, lying perpendicular to the longitudinal axis of the cell [85]. It's also been suggested that the number of basal feet can be changed in response to mechanical stimuli in order to modulate primary cilium stability [85]. Less speculatively, a connection between actin-myosin contractility and ciliogenesis has been shown, with inhibition of contractility on highly spread, non-ciliated cells leading to reorientation of the basal body and ciliogenesis [121]. Whether cilium fluctuations could have any connection to basal body/cilium orientational dynamics is highly speculative, but experiments observing cilium fluctuations during and after extended periods of loading, similar to our adaptation experiments, might provide clues about feedback between cilium position and cellular response.

\section{Outlook}

The primary cilium is a rich dynamical system. Though we have explored some of its mechanical and dynamical aspects in this work, more remains to be done. The hints of cilium stiffening under load reported in Ref. [85] suggest that the cilium has dynamical mechanical properties, which would be well-worth investigating. As already mentioned at the end of the last section, this could be done by looking at the mechanics of the cilium both before and after loading, via flow or optical trapdelivered forces. One result that deserves following up on is the double exponential behavior of the relaxation curves from the bend \& relax experiments detailed in Chapter 6. Specifically, the "slow tail", i.e. the slower exponential relaxation, may contain interesting information about the cilium elastic confinement/attachment to the cell. Figure 7.4 shows the "slow tail" from a bend \& relax experiment which wasn't included in our analysis because the cilium was bent through a large angle, violating the small curvature condition. Interestingly, in the slow exponential relaxation one can see numerous dips, indicated with black arrows, in which the cilium made a quick movement in the equilibrium direction, followed by a symmetric movement backwards. This is superimposed upon a plateau in the movement of several hundred milliseconds in which the cilium stays in the same position after the initial decay. This is followed by, at the end of the curve, another large movement towards the equilibrium position, coincident with a dip. These could represent the superposition of the natural cilium fluctuations on the decay, but the existence of a plateau, the "stop and go" motion mentioned in Chapter 6, is noteworthy. Data such as this suggest that the cilium recovery after extreme bending could contain interesting dynamics.

We have thus far only explored the connection of cilium fluctuations to actin- 


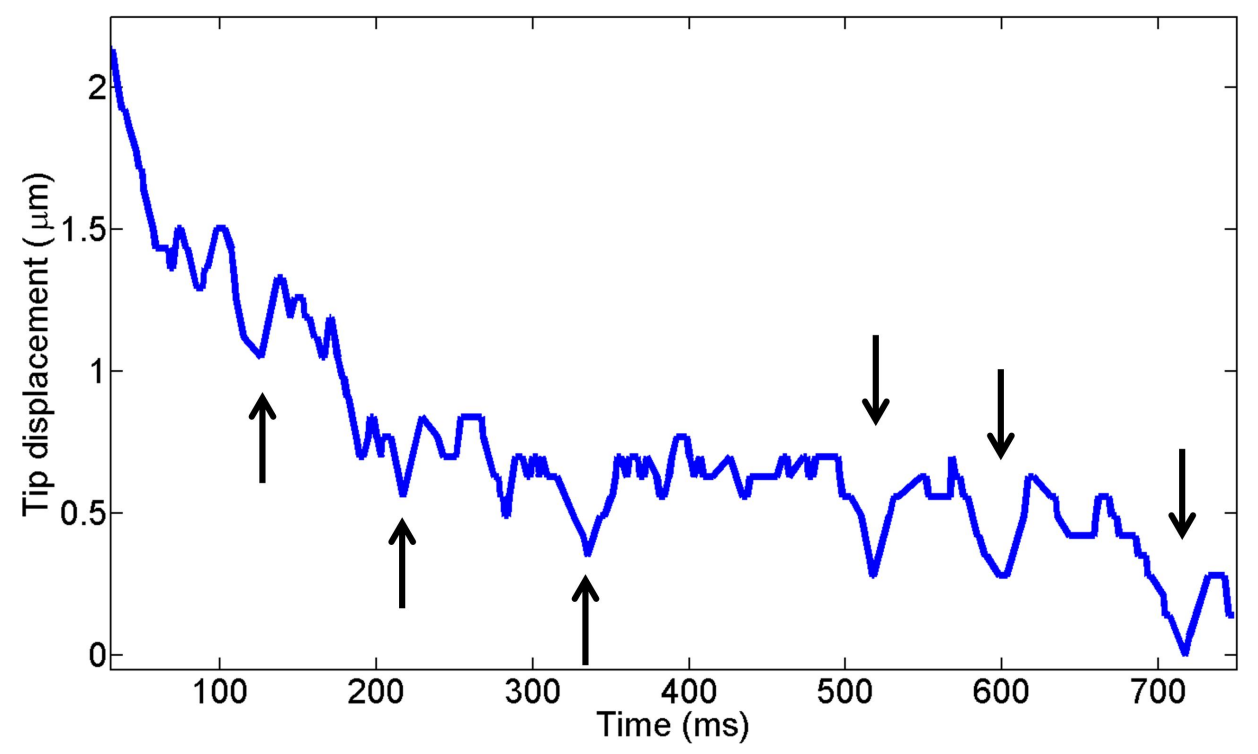

Figure 7.4: Cilium tip position vs. time. Depicted is a detail of the "slow tail" of a double exponential relaxation curve from a strongly bent cilium in a bend \& relax experiment. Superimposed on the overall decay are dips, indicated by the black arrows, resulting from a quick back and forth movement of the cilium.

myosin activity. Given the list of cytoskeletal elements which modulate cilium length and mechanotransduction, it would be interesting to determine what effect disruption of microtubules, cadherins, integrins, or cytoplasmic dynein might have on them. It may turn out that cilium fluctuations can be modulated by various pathways, though the agreement in the magnitude of fluctuations between blebbistatin treated and ATP-depleted cells points towards actin-myosin specificity.

Finally, it would be interesting to probe the mechanosensitive response of MDCK cells with optical traps directly, rather than with flow as has been done to date. With specific, local application of forces it would become possible to test whether or not cilium bending alone is sufficient for a mechanosensitive response and what the specific threshold force/curvature necessary to elicit a calcium response is (or whether such a sharp threshold exists). Optical trapping would also allow novel applications of force to the cilium, such as inducing tension in the ciliary membrane by pulling longitudinally outward along the cilium with an attached bead. In short, simultaneous trapping and calcium imaging would give the experimenter a wide range of different options to test the cilium's mechanosensitive response.

Fifteen years ago, the primary cilium was thought to be a vestigial organelle by most of the biological community, of no use to the cell despite its ubiquity. Since then an explosion in research has implicated the primary cilium in a huge number 
of cellular processes, from vision to mechanosensation to determination of left-right asymmetry. The impact of cilium defects on human health and its promiscuity as a cellular sensing antenna have made it an area of very active research in the medical and biological community. Despite this interest, biophysics studies have been slow in coming, with more attention paid to motile cilia and flagella. We hope that this work has helped fill in some of the gaps in our biophysical understanding of the primary cilium and that it has highlighted its potential as a rich system deserving of further research. 


\section{Part I}

Appendices 



\section{Appendix A}

\section{Protocols}

In this appendix we give step-by-step protocols for cell culture, cultivation on polycarbonate membranes (PCMs), bead coating, blebbistatin treatment, and ATPdepletion.

\section{Cell culture: Subcultivation of MDCK-II cells}

Cell line:

MDCK-II (Madin-Darby Canine Kidney)

Materials:

Culture medium: Earle's MEM (Biochrom AG, F0325) with 2 mM L-glutamine (Gibco, 25030), 1\% penicillin-streptomycin (Lonza, DE17-602E), and 10\% fetal bovine serum (Sigma F0804)

EDTA/Trypsin solution, 0.02/0.05\% respective concentrations (SAFC, 59417C).

T75 culture flask $\left(75 \mathrm{~cm}^{2}\right)$

Incubator at $37^{\circ} \mathrm{C}$ with a $5 \% \mathrm{CO}_{2}$ atmosphere

\section{Cell passaging protocol:}

1. Suck culture medium out of a flask containing $70-90 \%$ confluent cells.

2. Wash the cell sheet with 1-2 mL (assuming a T75 culture flask) EDTA/Trypsin solution. Suck out the solution.

3. Add 2-3 mL EDTA/Trypsin solution and incubate at $37^{\circ} \mathrm{C}$ for $5-15$ minutes (depends on level of confluence).

4. Add 4-5 mL of culture medium and gently wash the bottom of the flask several times to resuspend the cells. 
5. Put the cell solution in a Falcon tube and centrifuge for 5 minutes at 1000 rpm.

6. Suck out the solution being careful not to disturb the cell pellet at the bottom of the tube.

7. Add $1 \mathrm{~mL}$ of culture medium to the cell pellet and resuspend cells.

8. Count the cells.

9. Add the desired number of cells to a new culture flask with $10 \mathrm{~mL}$ of culture medium and incubate. 


\section{Cell cultivation on PCMs}

\section{Materials:}

Polycarbonate membranes (Whatman Nuclepore, 110607, $400 \mathrm{~nm}$ pore size)

Poly-L-lysine, 0.01\% sterile-filtered solution (Sigma, P4707)

6-well culture plate

\section{PCM cultivation protocol:}

1. Put one PCM in each 6-well dish and place under UV light for 1 hour to sterilize.

2. Add $0.5-1 \mathrm{~mL}$ of poly-L-lysine to each well and incubate the PCMs for 10-30 minutes at room temperature.

3. Suck poly-l-lysine solution out of the wells.

4. Subcultivate the cells as described in the preceding protocol through the cell counting step.

5. Add $\sim 150,000$ cells to each membrane and then fill each 6 -well with $2 \mathrm{~mL}$ of culture medium.

6. Incubate the 6 -well at $37^{\circ} \mathrm{C}$.

7. Check the cells after 24 hours. If a significant number of cells have started to grow on the 6-well bottom, move the PCMs carefully with tweezers to a fresh 6-well, add medium, and incubate. 


\section{Bead antibody coating}

\section{Materials:}

$1.5 \mu \mathrm{m}$ carboxylated silica beads, diluted 1:10 from $50 \mathrm{mg} / \mathrm{mL}$ stock solution (Kisker Biotech GmbH, PSi-1.5COOH)

PC1-antibody (Abcam PLC, Anti-Polycystin 1 antibody, [7e12], ab74115)

Protein-G from Streptococcus sp., $5 \mathrm{mg} / \mathrm{mL}$ in $\mathrm{H}_{2} \mathrm{O}$ (Fluka 08062)

Dulbecco's phosphate buffered solution (Sigma D8537)

Note: The original protocol used plain silica beads rather than carboxylated ones.

\section{Bead antibody coating protocol:}

1. Add $10 \mu \mathrm{L}$ of bead solution to $80 \mu \mathrm{L}$ of PBS. Add $10 \mu \mathrm{L}$ of Protein-G to this solution.

2. Incubate the solution at $4^{\circ} \mathrm{C}$ for 30 minutes.

3. Centrifuge at $10000 \mathrm{rpm}$ at room temperature for 5 minutes.

4. Suck out the fluid being careful not to disturb the sedimented pellet of beads at the bottom.

5. Resuspend the pellet in $90 \mu \mathrm{L}$ of PBS. Add $10 \mu \mathrm{L}$ of antibody solution.

6. Incubate the solution at $4^{\circ} \mathrm{C}$ for 30 minutes.

7. Centrifuge at $10000 \mathrm{rpm}$ at room temperature for 5 minutes.

8. Suck out the fluid being careful not to disturb the sedimented pellet of beads at the bottom.

9. Resuspend the pellet in $100 \mu \mathrm{L}$ of PBS. 


\section{Blebbistatin treatment protocol}

Materials:

50 mM Blebbistatin, Racemic (CalBiochem, InSolution, 203389)

MDCK II cells cultured on a PCM membrane in a 6-well

Note: Only the (-) enantiomer of Blebbistatin inhibits myosin II motor activity. We use $100 \mu \mathrm{M}$ of a racemic solution, which translates into only $50 \mu \mathrm{M}$ of the (-) enantiomer for inhibitory purposes.

1. Add Blebbistatin to each well to reach a concentration of $100 \mu \mathrm{M}(4 \mu \mathrm{L}$ to $2 \mathrm{~mL}$ of culture medium).

2. Incubate cells at $37^{\circ} \mathrm{C}$ for 30 minutes.

3. Mount cells in chamber for viewing with solution containing $100 \mu \mathrm{M}$ Blebbistatin. 


\section{ATP-depletion protocol}

Materials:

Dulbecco's MEM without D-glucose (Biochrom AG, F0405) with 2 mM L-glutamine (Gibco, 25030)

Antimycin A from Streptomyces sp. (Sigma, A8674-25MG)

2-Deoxy-D-glucose (Sigma, D8375-1G)

MDCK II cells cultured on a PCM membrane in a 6-well

1. Replace the culture medium in the 6-well with the glucose-free Dulbecco's MEM solution.

2. Incubate cells at $37^{\circ} \mathrm{C}$ for 3 hours.

3. Add Antimycin A to a concentration of $10 \mu \mathrm{M}$ and 2-Deoxy-D-glucose to a conecntration of $10 \mathrm{mM}$ to the Dulbecco's MEM solution.

4. Incubate cells at $37^{\circ} \mathrm{C}$ for $10-15$ minutes.

5. Mount cells in chamber for viewing with glucoes-free solution containing $10 \mu \mathrm{M}$ Antimycin A and 10 mM 2-Deoxy-D-glucose. 


\section{Appendix B}

\section{Influence of DIC prism orientation on crosstalk}

In order to establish that beam splitting was not occurring at a DIC prism orientation of $0^{\circ}$ (see Chapter 3), we measured the amount of crosstalk between the $s$ - and $p$-polarization detection channels. Beams which are $s$ - or $p$-polarized become elliptically polarized when traversing a prism oriented at $45^{\circ}$ and traverse the sample separated by the shear distance of the prism. The two beams encounter sample-dependent optical path differences which are usually unequal, inducing a phase difference between the two beams. When the beams are recombined by the condenser Wollaston prism this phase mismatch leads to imperfect separation of the beams into their original $s$ - or $p$-components, with some portion of the $p$-beam becoming $s$-polarized and vice versa. This phenomenon leads to cross talk between the two channels. We measure the amount of cross talk between the channels in two ways. First, we turn on only the $p$-polarized beam, trap a bead (1 $\mu \mathrm{m} \mathrm{Si})$, and measure the amount of integrated power that shows up in the $s$-channel. With a polarizer incorporated into the s-channel to clean up depolarization from other optical elements (see Reference [125]), we see $2.0 \pm 0.2 \%$ of the total power in the $s$-channel with no DIC prisms in the beam line, comparable to Ref. [125]. When we insert both DIC prisms at $45^{\circ}$ with respect to the $p$-polarized beam, we measure $4.5 \pm 0.4 \%$ of the total power in the $s$-channel. Upon rotating both prisms to a $0^{\circ}$ orientation with respect to the $p$-polarized beam the amount of power ending up in the $s$-channel goes down to $2.2 \pm 0.3 \%$.

Cross talk also shows up in the data as the erroneous signal of a particle trapped in one beam in the "wrong" detection channel. To measure this effect, we turned on both the $s$ - and $p$-polarized beams and trapped a bead $(1.5 \mu \mathrm{m} \mathrm{Si})$ with the $p$-beam while leaving the $s$-trap empty. Laser power was adjusted to be equal in both traps. We then calculated the power spectral densities (PSDs) from the signal recorded on each QPD. The results are shown in Figure B.1. In the occupied trap 


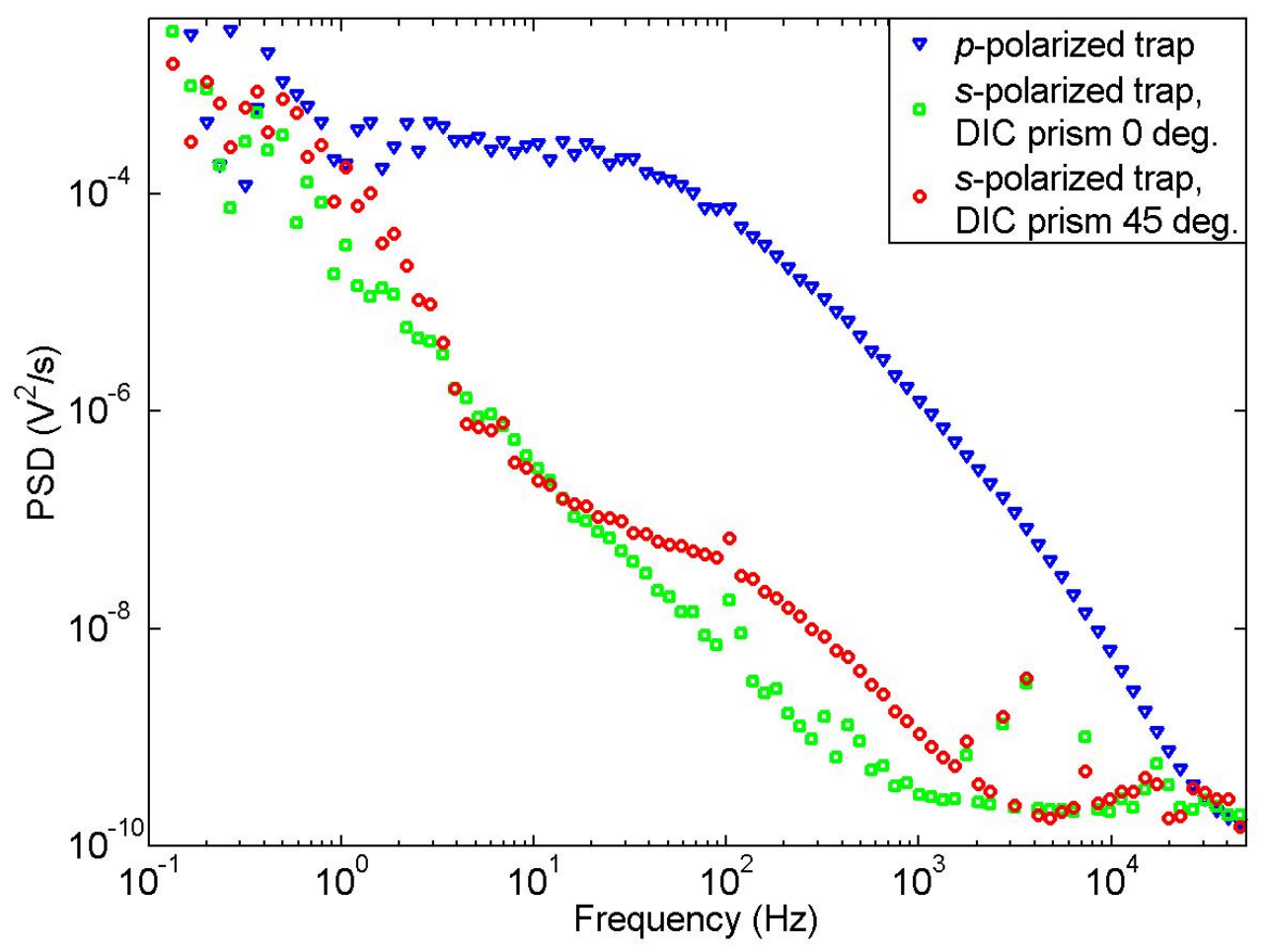

Figure B.1: PSDs of the fluctuations of a $1.5 \mu \mathrm{m}$ Si silica bead trapped in the $p$-polarized trap (blue triangles) and of the empty $s$-polarized trap with the DIC prisms rotated to either $0^{\circ}$ or $45^{\circ}$ (green squares and red circles, respectively). When the DIC prism is oriented at $45^{\circ}$ with respect to the orthogonally polarized $s$ - and $p$-beams, beam splitting leads to cross talk apparent as an attenuated Lorentzian in the empty $s$-channel. When the DIC prism is oriented at $0^{\circ}$, the cross talk is mitigated and the attenuated Lorentzian disappears.

one clearly sees the characteristic Lorentzian PSD spectrum expected for a trapped bead [125]. When the DIC prism is oriented at $45^{\circ}$, one sees an attenuated version of the Lorentzian in the empty trap due to cross talk between the beams. Rotating the prism to a $0^{\circ}$ orientation eliminates the cross talk in the s-channel and with it the attenuated Lorentzian signal. 


\section{Bibliography}

[1] N.F. Berbari et al., Curr. Biol. 19, R526 (2009).

[2] G. Albrecht-Buehler and A. Bushnell, Exp. Cell Res. 126, 427 (1980).

[3] T. Movassagh et al., Nat. Struct. Mol. Biol. 17, 761 (2010).

[4] N.F. Berbari et al., Proc. Natl. Acd. Sci. 105, 4242 (2008).

[5] G.J. Pazour et al., Curr. Biol. 12, R1 (2002).

[6] N.S. Murcia et al., Development 127, 2347 (2000).

[7] I.R. Veland et al., Nephron Physiol. 111, 39 (2009).

[8] G.J. Pazour and G. B. Witman, Curr. Opin. Cell Biol. 15, 105 (2003).

[9] C. Ott et al., Cilia 1, 3 (2012).

[10] T. Nagai et al., Proc. Natl. Acd. Sci. 101, 10554 (2004).

[11] V. Singla and J.F. Reiter, Science 313629 (2006).

[12] H.A. Praetorius and K.R. Spring, J. Membr. Biol. 18471 (2001).

[13] H.A. Praetorius and K.R. Spring, J. Membr. Biol. 19169 (2002).

[14] S.M. Nauli et al., Nat. Genet. 33, 129 (2003).

[15] H.F. Cantiello, Am. J. Physiol. Renal Physiol. 286, 1012 (2004).

[16] A. Giamarchi et al., EMBO Reports 7, 787 (2006).

[17] E.A. Schwartz et al., Am. J. Physiol. Renal Physiol. 272, F132 (1997).

[18] M.E. Downs et al., Comput. Methods Biomech. Biomed. Eng., DOI:10.1080/10255842.2011.653784 (2012).

[19] Y.N. Young et al., Biophys. J. 103, 629 (2012). 
[20] B. Sakmann and E. Neher, Single-Channel Recording, 2nd Ed. (Plenum Press, New York and London, 1995).

[21] A. Ashkin and J.M. Dziedzic, Science 235, 1517 (1986).

[22] C. Veigel et al., Nat. Cell Biol. 4, 59 (2002).

[23] M.W. Allersma et al., Biophys. J. 74, 1074 (1998).

[24] A.D. Mehta et al., Science 283, 1689 (1999).

[25] C. Bustamante et al., Nature 421, 423 (2003).

[26] D. Mizuno et al., Science 315370 (2007).

[27] V. Pelletier et al., Phys. Rev. Lett. 102, 188303 (2009).

[28] T. Toyoyta et al., Soft Matter 7, 3234 (2011).

[29] A. Alemany et al., Nature Phys. 8, 688 (2012).

[30] K.C. Neumann and S.M. Block, Rev. Sci. Instrum. 75, 2787 (2004).

[31] K. Svoboda and S.M. Block, Annu. Rev. Biophys. Biomol. Struct. 23, 247 (1994).

[32] A. Ashkin, Biophys. J 61, 569 (1992).

[33] N.B. Simpson et al., J. Mod. Optics 45, 1943 (1998).

[34] A. Sischka et al., Rev. Sci. Instrum. 79, 063702 (2008).

[35] A. Rohrbach and E.H.K. Steltzer, J. Appl. Phys.91, 5474 (2002).

[36] A. Mazolli et al., Proc. R. Soc. Lond. A 459, 3021 (2003).

[37] P.A.M. Neto and H.M. Nussenzveig, Europhys. Lett. 50702 (2008).

[38] M. Mahamdeh et al., Opt. Express 19, 11759 (2011).

[39] X. Micahlet et al., Annu. Rev. Biophys. Biomol. Struct. 32, 161 (2003).

[40] J.W. Lichtmann and J.A. Conchello, Nat. Methods 2, 910 (2005).

[41] M. Bixon and J. Jortner, J. Chem. Phys. 48, 715 (1968).

[42] M. Born and E. Wolf, Principles of Optics, 7th Ed. (Cambridge University Press, New York, 1999). 
[43] P. Debye and F.W. Sears, Proc. Natl. Acad. Sci. 18, 409 (1932).

[44] D. Brewster, Phil. Trans. R. Soc. Lond. 106, 156 (1816).

[45] N. Uchida and N. Nizeki, Proc. IEEE, 61, 1073 (1973).

[46] C.S. Brown et al., Proc. IEEE, 109, 99 (1962).

[47] Physik Instumente GmbH

"Fundamentals of Piezoelectricity"

http://www.physikinstrumente.com/en/products/prdetail.php?sortnr=400600.00

[48] J. van Mameren, Single molecule mechanics of biopolymers: An optical tweezers study, MSc. Thesis, Vrije Universiteit, Amsterdam (2002).

[49] F. Gittes and C.F. Schmidt, Opt. Lett. 23, 7 (1998).

[50] J.H.G. Huisstede et al., Opt. Express 13, 1113 (2005).

[51] A.R. Carter et al., Opt. Express 15, 13434 (2007).

[52] J.K. Dreyer et al., Appl. Opt. 43, 1991 (2004).

[53] C. Deufel and M.D. Wang, Biophys. J. 90, 657 (2006).

[54] A. Samadi and S.N.S. Reihani, Opt. Lett. 36, 4056 (2011).

[55] R.W. Boyd, J. Opt. Soc. Am. 70, 877 (1980).

[56] V. Bormuth et al., Opt. Express 16, 13831 (2008).

[57] I.M. Tolić-Nørrelykke et al., Comput. Phys Commun. 159, 225 (2004).

[58] K. Berg-Sørensen et al., Rev. Sci. Instrum. 77, 063106 (2006).

[59] Nikon Microscopy U "de Sénarmont Bias Retardation in DIC Microscopy" http://www.microscopyu.com/articles/dic/desenarmontdicintro.html

[60] UC Berkeley Biological Imaging Facility "Differential interference contrast (DIC)" http://microscopy.berkeley.edu/Resources/instruction/DIC.html

[61] T. Kim et al., Opt. Express 20, 6737 (2012).

[62] Olympus Microscopy Resource Center, "Fundamental Concepts in DIC Microscopy" http://www.olympusmicro.com/primer/techniques/dic/dicintro.html 
[63] W. Lichten, J. Opt. Soc. Am. A 2, 1869 (1985).

[64] V. Bormuth et al., J. Microsc. 2261 (2007).

[65] S. Inuoé, Video Microscopy (Plenum Press, New York, 1986).

[66] A. Gerson-Gurwitz et al., EMBO Journal 304942 (2011).

[67] Nikon Microscopy U "Specimen Contrast in Optical Microscopy" http://www.microscopyu.com/articles/formulas/specimencontrast.html

[68] G. Danuser et al., J. Microsc. 19834 (2000).

[69] W.H. Bosking et al., J. Neurosci. 17, 2112 (1997).

[70] J. Canny, IEEE Trans. Pattern Anal. Mach. Intell. 8, 679 (1986).

[71] D. Sage et al., IEEE Trans. Image Processing 141372 (2005).

[72] K.E. Roth et al., J. Cell Sci. 89, 457 (1988).

[73] V. Babich et al., J. Biol. Chem. 2425582 (2004).

[74] R.P. Feynman, Feynman Lectures on Physics, Vol. II (AddisonWesley, Boston,1970).

[75] S.M. Nauli et al., Meth. Enzymol. 525, 1 (2013).

[76] S. Rydholm et al., Am. J. Physiol. Renal Physiol. 298, 1096 (2010).

[77] A. Resnick, J. Biomed. Opt. 15, 015005 (2010).

[78] H. Felgner et al., J. Cell Sci. 109, 509 (1996).

[79] C.H. Wiggins et al., Biophys. J. 74, 1043 (1998).

[80] F. Gittes et al., J. Cell. Biol. 120, 923 (1993).

[81] F. Pampaloni et al., Proc. Natl. Acad. Sci. 103, 10248 (2005).

[82] S.C. Weber et al., Proc. Natl. Acad. Sci. 109, 7338 (2011).

[83] R. Bacallao et al., J. Cell Sci. 107, 3301 (1994).

[84] B. Yoder (Ed.), Ciliary Function in Mammalian Development (Elsevier, Oxford, 2008). 
[85] D.A. Hoey et al., J. Biomech. 4517 (2012).

[86] T.W. Ridler and S. Calvard, IEEE Trans. Syst., Man, Cybern. 8, 630 (1978).

[87] K.F. Mulchrone and K.R. Choudhury, J. Struct. Geol. 26, 143 (2004).

[88] F. Rehfeldt, (Private Communication).

[89] S. Yoshiba et al., Science 338, 226 (2012).

[90] J.L. Rodgers and W.A. Nicewander, Am. Stat. 42, 59 (1988).

[91] X.Y. Wang et al., Am. J. Physiol. 267, F1007 (1994).

[92] C. Delles et al., J. Physiol. 486.3, 557 (1995).

[93] T. Haller et al., Biochem. J. 319, 909 (1996).

[94] P. Dietl et al., Cell Calcium 20, 11 (1996).

[95] T. Jsepersen et al., J. Cell Sci. 117, 4517 (2004).

[96] S. Hsu and R.R. Burnette, Biochim. Biophys. Acta 1329, 26 (1997).

[97] M.N. Helms et al., J. Biol. Chem. 280, 40885 (2005).

[98] O.N. Kovbasnjuk and K.R. Spring, J. Membr. Biol. 17619 (2000).

[99] The liquid junction potential was estimated using the Junction Potential Calculator implemented in Clampex (Molecular Devices, Sunnyvale, CA).

[100] I. Ibañez-Tallon et al., Hum. Mol. Gen. 12, R27 (2003).

[101] J. Lin et al., J. Biol. Chem. 28629175 (2011).

[102] C.K. Omoto et al., Mol. Biol. Cell 10, 1 (1999).

[103] P. Yang et al., J. Cell Sci. 119, 1166 (2005).

[104] M.G.L. van den Heuvel et al., Nano Lett. 7, 3138 (2007).

[105] P. Venier et al., J. Biol. Chem. 269, 13353 (1994).

[106] J. van Mameren et al., J. Phys. Chem. B 113, 3837 (2009).

[107] B. Mickey and J. Howard, J. Cell Biol. 130, 909 (1995). 
[108] M. Dogterom and B. Yurke, Science 278, 856 (1997).

[109] C. Heussinger et al., Phys. Rev. E 81, 021904 (2010).

[110] M. Okuno and Y. Hiramoto, J. Exp. Biol. 79, 235 (1979).

[111] S. Ishijima and Y. Hiramoto, Cell Struct. Funct. 19, 349 (1994).

[112] C.B. Lindemann et al., Biophys. J. 13, 437 (1973).

[113] E. Gluenz et al., FASEB Journal 243117 (2010).

[114] M. Zhang et al., Proc. Natl. Acad. Sci. 101, 2311 (2004).

[115] M. Bornens, Nat. Rev. Mol. Cell Biol. 9, 875 (2008).

[116] H. Hagiwara et al., J. Anat. 200, 89 (2002).

[117] F.J. Alenghat et al., Exp. Cell Res. 301, 23 (2004).

[118] J. Kim et al., Nature 464, 1048 (2010).

[119] K. Miyoshi et al., Acta Med. Okayama 65, 279 (2011).

[120] N. Sharma et al., Mol. Biol. Cell 22, 806 (2011).

[121] A. Pitaval et al., J. Cell Biol. 191, 303 (2010).

[122] H. Ishikawa, (Private Communication).

[123] J.K. Douglass et al., Nature 365, 337 (1993).

[124] S.M. Bezrukov and I Vodyanoy, Nature 378, 362 (1995).

[125] M. Atakhorrami et al., Rev. Sci. Instrum. 79, 043103 (2008). 


\title{
Christopher BATtLE
}

\section{Personal Data}

\author{
Place And Date of Birth: Canada, 24 March 1978 \\ NATIONALITY: American \\ ADDRESS: Jüdenstraße 35, Göttingen, Germany \\ Phone: +49176 79718626 \\ EMAIL: cbattle12@yahoo.com
}

\section{Education}

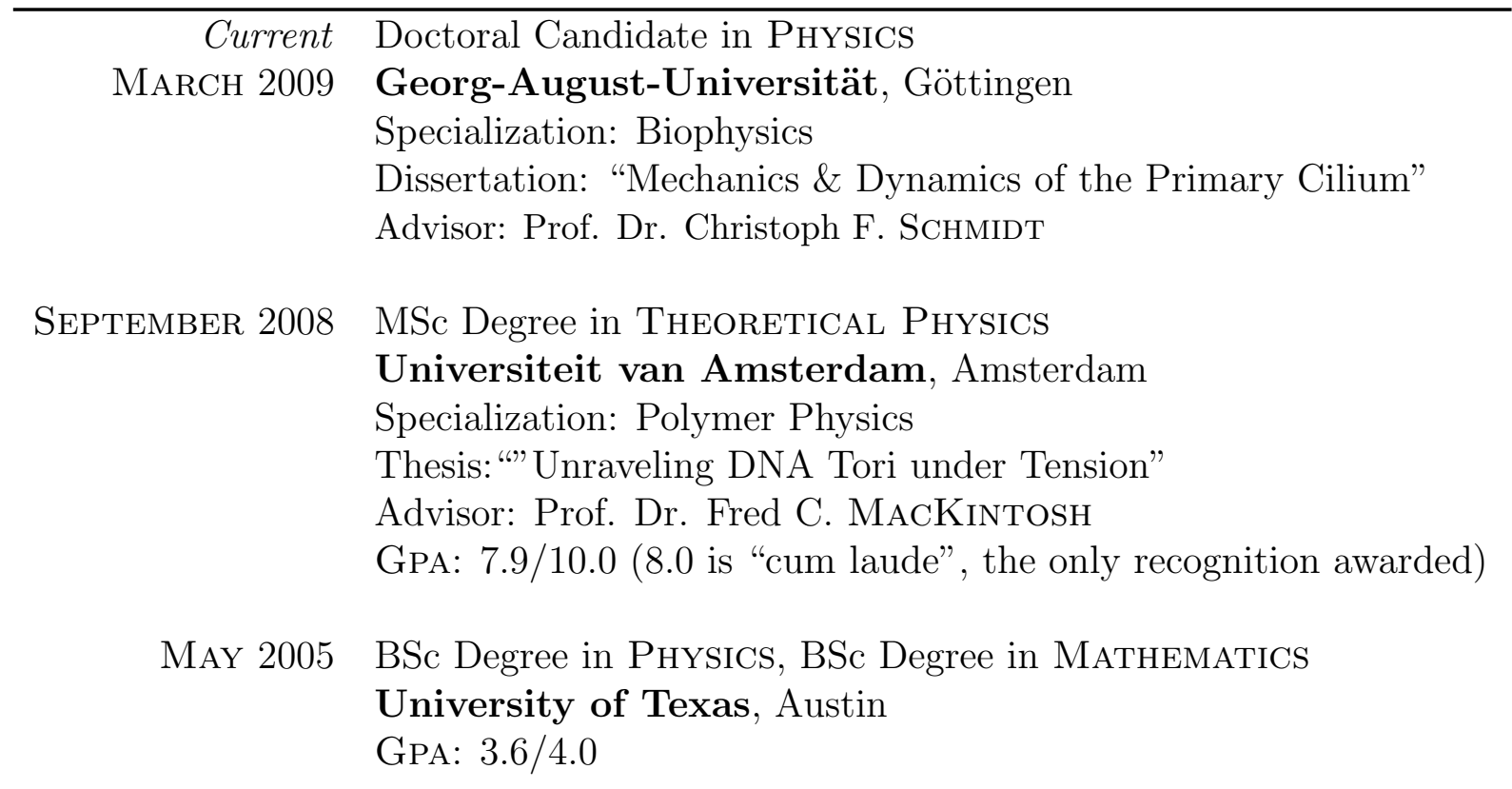

\section{Work Experience}

\begin{tabular}{l|l} 
Aug 2000- May 2005 & Technician at High Energy Physics Group, Texas A\&M
\end{tabular} Univ. and Univ. of Texas at Austin

Built detector system and programmed hardware/software interface in LabView. Performed quality control, performance, and helium-induced failure measurements of electronics to be used in the MINOS experiment. Manufactured stress transducers to be used in experimental block-coil superconducting magnets and assisted in magnet winding. Tested release mold performances on the coil segments. Worked on PRL publication on rare decays in CDF data and EM timing proposal for the CDF detector. 


\section{Scholarships and Certificates}

\begin{aligned} \hline APR 2009- APR 2011 & IMPRS Stipend recipient \\ SEPT 2006- SEPT 2008 & IFTA Scholarship \\ SEPT 2006- SEPT 2008 & Amsterdam Merit Scholarship \\ AUG 2002- MAY 2005 & Honor roll \& Dean's Merit List every semester \\ JAN 2004- JAN 2005 & Reiger Scholarship \\ DEC 2004 & GRE: $1560 / 1600$ (Q:780,V:780) 99 ${ }^{\text {th }}$ percentile \end{aligned}

\section{Publications}

\begin{tabular}{|c|c|}
\hline FIRST AUTHOR & $\begin{array}{l}\text { "Differential interference contrast microscopy using light- } \\
\text { emitting diode illumination in conjunction with dual optical } \\
\text { traps", C. Battle et al., accepted to Rev. Sci. Instrum. } \\
\text { "Unraveling DNA tori under tension", C. Battle et al., 2009, } \\
\text { Phys. Rev. E } \mathbf{8 0}\end{array}$ \\
\hline
\end{tabular}

Co-Author "Visualizing the Formation and Collapse of DNA Toroids", B. van den Broek et al., 2010 Biophys. J. 98

"Search for new heavy particles in the $\mathrm{W} \mathrm{Z}_{0}$ final state in $\mathrm{p}$ anti-p collisions at $\mathrm{s}^{1 / 2}=1.8 \mathrm{TeV}$ ", A. A. Affolder et al., 2002, Phys. Rev. Lett. 88

"Testing of TAMU1: A single-aperture block-coil dipole", R. Benjegerdes et al., 2001 Proc. of Part. Accel. Conf.

"Optimization of block-coil dipoles for hadron colliders", C.Battle et al., 1999 Proc. of Part. Accel. Conf.

\section{Languages}

\begin{aligned} \hline ENGLISH: & Native Speaker \\ GERMAN: & Fluent \\ SpAnish: & Basic Knowledge \end{aligned}

\section{Interests and Activities}

Music (Performance \& Composition), Writing, Metaphysics, Performance Art, Traveling 